\title{
$\alpha, \alpha^{\prime}-\mathrm{C}-\mathrm{H}-$ Bond Difunctionalization of Unprotected Alicyclic Amines
}

\author{
Daniel A. Valles, ${ }^{1}$ Subhradeep Dutta, ${ }^{1}$ Anirudra Paul, ${ }^{1}$ Khalil A. Abboud,${ }^{2}$ Ion Ghiviriga, ${ }^{3}$ \\ and Daniel Seidel ${ }^{1, *}$ \\ ${ }^{1}$ Center for Heterocyclic Compounds, Department of Chemistry, University of Florida, Gainesville, \\ Florida 32611, United States \\ ${ }^{2}$ Center for X-Ray Crystallography, Department of Chemistry, University of Florida, Gainesville, Florida \\ 32611, United States \\ ${ }^{3}$ Center for NMR Spectroscopy, Department of Chemistry, University of Florida, Gainesville, Florida \\ 32611, United States \\ * Correspondence to: seidel@chem.ufl.edu
}

\section{Supporting Information}

\section{Table of Contents}

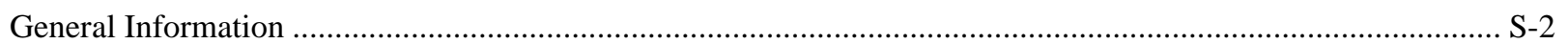

Evaluation of Reaction Conditions for the $\alpha, \alpha^{\prime}$-Difunctionalization of Secondary Alicyclic Amines ................... S-3

General Procedures A and B for the $\alpha, \alpha^{\prime}$-Difunctionalization of Secondary Alicyclic Amines............................. S-7

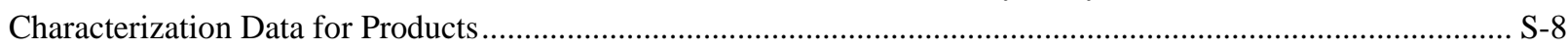

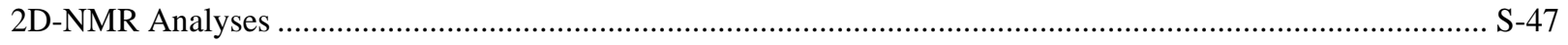

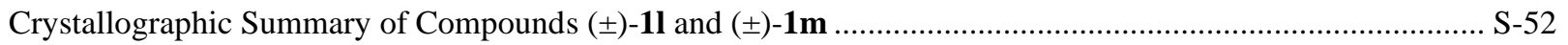

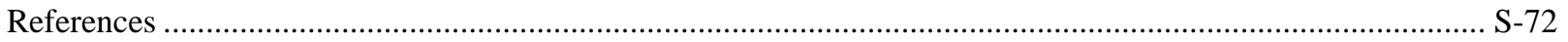

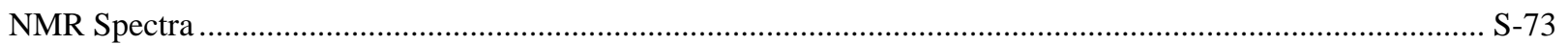


General Information: Starting materials and reagents were purchased from commercial sources and used as received unless stated otherwise. Anhydrous diethyl ether was dried using a JC Meyer solvent system. All liquid amines, liquid bromobenzenes, thiophene, trifluoroacetophenone, and phenyl $t$-butyl ketone were distilled prior to use. $n$-Butyl lithium solution in hexanes, phenyl lithium solution in dibutyl ether, and $n$-hexyl lithium solution in hexanes were purchased from commercial sources and freshly titrated using $N$-pivaloyl-o-toluidine prior to use. ${ }^{1}$ Purification of reaction products was carried out by flash column chromatography using Sorbent Technologies Standard Grade silica gel (60 ̊, 230-400 mesh). Analytical thin layer chromatography was performed on EM Reagent $0.25 \mathrm{~mm}$ silica gel 60 F254 plates. Visualization was accomplished with UV light, Dragendorff-Munier stains, followed by heating. Melting points were recorded on an Electrothermal Digital Mel-Tempt 3.0 melting point apparatus. Proton nuclear magnetic resonance spectra ( ${ }^{1} \mathrm{H}$ NMR) were recorded on a Mercury $300 \mathrm{MHz}$, a Varian Unity Inova $500 \mathrm{MHz}$, a Bruker $400 \mathrm{MHz}$, or a Bruker $600 \mathrm{MHz}$ instrument and chemical shifts are reported in ppm using the solvent as an internal standard $\left(\mathrm{CDCl}_{3}\right.$ at $\left.7.26 \mathrm{ppm}\right)$. Data are reported as app = apparent, $\mathrm{s}=$ singlet, $\mathrm{d}=$ doublet, $\mathrm{t}=$ triplet, $\mathrm{q}=$ quartet, $\mathrm{m}=$ multiplet, comp = complex, $\mathrm{br}=$ broad; coupling constant $(\mathrm{s})$ in $\mathrm{Hz}$. Proton-decoupled carbon nuclear magnetic resonance spectra $\left({ }^{13} \mathrm{C}\right.$ NMR) spectra were recorded on a Mercury 300 MHz, a Varian Unity Inova $500 \mathrm{MHz}$, a Bruker $400 \mathrm{MHz}$, or a Bruker $600 \mathrm{MHz}$ instrument and chemical shifts are reported in ppm using the solvent as an internal standard $\left(\mathrm{CDCl}_{3}\right.$ at $\left.77.0 \mathrm{ppm}\right)$. GCOSY and NOESY spectra were recorded on a Varian Unity Inova $500 \mathrm{MHz}$ instrument. High resolution mass spectra (HRMS) were obtained from an Agilent 6230 ESI-TOF instrument. Benzyl lithium ${ }^{2}$ and other aryl lithium reagents ${ }^{3}$ other than phenyl lithium were prepared according to literature procedures. 


\section{Evaluation of Reaction Conditions for the $\alpha, \alpha$ '-Difunctionalization of Pyrrolidine. ${ }^{\text {a }}$}
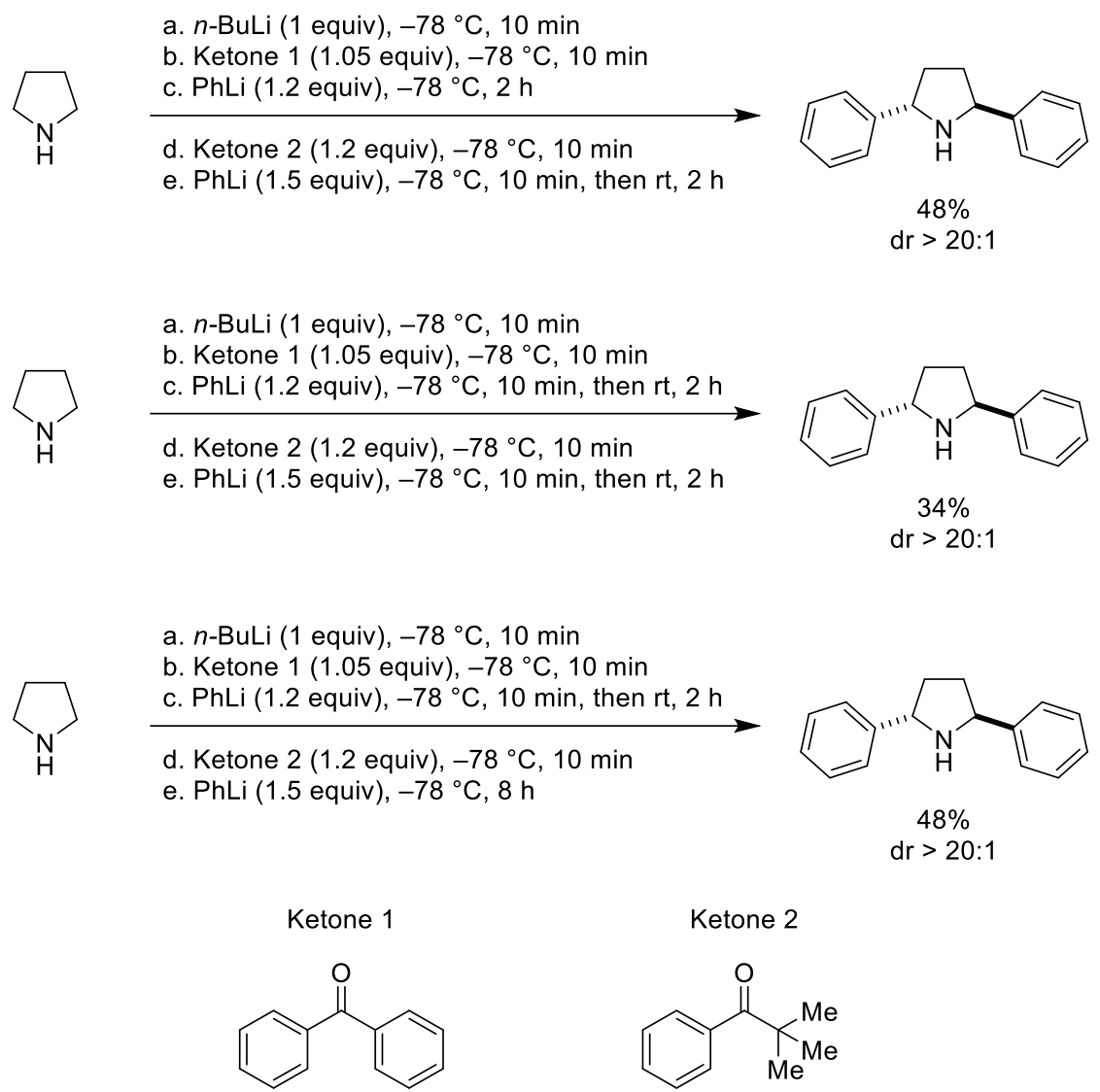

Ketone 2

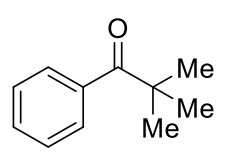

${ }^{a}$ Reactions were performed with $1 \mathrm{mmol}$ of the amine. Yields correspond to isolated product. 


\section{Evaluation of Reaction Conditions for the $\alpha, \alpha$ '-Difunctionalization of Piperidine. ${ }^{a}$}

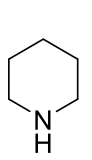

a. $n$-BuLi ( 1 equiv), $-78{ }^{\circ} \mathrm{C}, 10 \mathrm{~min}$

b. Ketone 1 ( 1.05 equiv), $-78^{\circ} \mathrm{C}, 10 \mathrm{~min}$

c. PhLi ( 1.2 equiv), $-78^{\circ} \mathrm{C}, 2 \mathrm{~h}$

d. Ketone 1 ( 1.7 equiv), $-78^{\circ} \mathrm{C}, 10 \mathrm{~min}$

e. PhLi (2 equiv), $-78^{\circ} \mathrm{C}, 10 \mathrm{~min}$, then $\mathrm{rt}, 2 \mathrm{~h}$

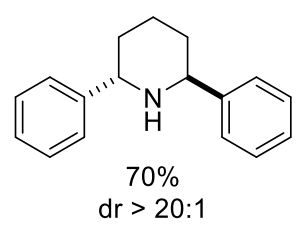

a. $n$-BuLi ( 1 equiv), $-78^{\circ} \mathrm{C}, 10 \mathrm{~min}$

b. Ketone 1 (1.05 equiv), $-78^{\circ} \mathrm{C}, 10 \mathrm{~min}$

c. $\mathrm{PhLi}$ (1.2 equiv), $-78^{\circ} \mathrm{C}, 2 \mathrm{~h}$

${ }_{\mathrm{H}}$

d. Ketone 1 ( 1.7 equiv), $-78^{\circ} \mathrm{C}, 10 \mathrm{~min}$

e.PhLi ( 2 equiv), $-78^{\circ} \mathrm{C}, 8 \mathrm{~h}$

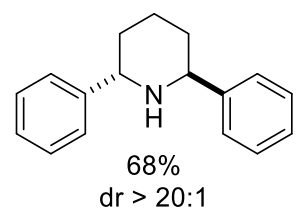

a. $n$-BuLi ( 1 equiv), $-78^{\circ} \mathrm{C}, 10 \mathrm{~min}$

b. Ketone 1 ( 1.05 equiv), $-78^{\circ} \mathrm{C}, 10 \mathrm{~min}$

c. PhLi ( 1.07 equiv), $-78^{\circ} \mathrm{C}, 2 \mathrm{~h}$

d. Ketone 1 ( 1.7 equiv), $-78^{\circ} \mathrm{C}, 10 \mathrm{~min}$

e. $n$-BuLi (2 equiv), $-78^{\circ} \mathrm{C}, 2 \mathrm{~h}$

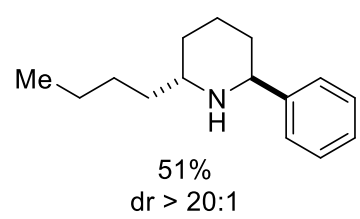

a. $n$-BuLi ( 1 equiv), $-78^{\circ} \mathrm{C}, 10 \mathrm{~min}$

b. Ketone 1 ( 1.05 equiv), $-78^{\circ} \mathrm{C}, 10 \mathrm{~min}$

c. PhLi (1.07 equiv), $-78^{\circ} \mathrm{C}, 10 \mathrm{~min}$, then $\mathrm{rt}, 2 \mathrm{~h}$

d. Ketone 1 ( 1.7 equiv), $-78^{\circ} \mathrm{C}, 10 \mathrm{~min}$

e. $n$-BuLi ( 2 equiv), $-78^{\circ} \mathrm{C}, 2 \mathrm{~h}$

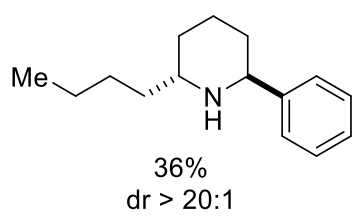

a. $n$-BuLi ( 1 equiv), $-78^{\circ} \mathrm{C}, 10 \mathrm{~min}$

b. Ketone 1 ( 1.05 equiv), $-78^{\circ} \mathrm{C}, 10 \mathrm{~min}$

c. PhLi (1.07 equiv), $-78{ }^{\circ} \mathrm{C}, 10 \mathrm{~min}$, then $\mathrm{rt}, 2 \mathrm{~h}$

d. Ketone 1 ( 1.7 equiv), $-78^{\circ} \mathrm{C}, 10 \mathrm{~min}$

e. $n$-BuLi (2 equiv), $-78^{\circ} \mathrm{C}, 10 \mathrm{~min}$, then $\mathrm{rt}, 2 \mathrm{~h}$

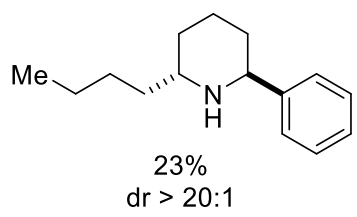

Ketone 1<smiles>O=C(c1ccccc1)C(F)(F)F</smiles>

${ }^{\text {a }}$ Reactions were performed with $1 \mathrm{mmol}$ of the amine. Yields correspond to isolated product. 


\section{Evaluation of Reaction Conditions for the $\alpha, \alpha^{\prime}$-Difunctionalization of Azepane. ${ }^{\text {a }}$}

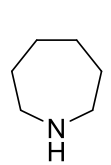

a. $n$-BuLi ( 1 equiv), $-78^{\circ} \mathrm{C}, 10 \mathrm{~min}$

b. Ketone 1 ( 1.05 equiv), $-78^{\circ} \mathrm{C}, 10 \mathrm{~min}$

c. PhLi ( 1.2 equiv), $-78^{\circ} \mathrm{C}, 2 \mathrm{~h}$

d. Ketone 2 (1.2 equiv), $-78^{\circ} \mathrm{C}, 10 \mathrm{~min}$

e. PhLi ( 1.5 equiv), $-78^{\circ} \mathrm{C}, 10 \mathrm{~min}$, then $\mathrm{rt}, 2 \mathrm{~h}$

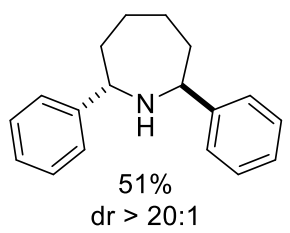

a. $n$-BuLi (1 equiv), $-78^{\circ} \mathrm{C}, 10 \mathrm{~min}$

b. Ketone 1 (1.05 equiv), $-78^{\circ} \mathrm{C}, 10 \mathrm{~min}$

c. PhLi (1.2 equiv), $-78^{\circ} \mathrm{C}, 2 \mathrm{~h}$

d. Ketone 1 ( 1.2 equiv), $-78^{\circ} \mathrm{C}, 10 \mathrm{~min}$

e. PhLi ( 1.5 equiv), $-78{ }^{\circ} \mathrm{C}, 10 \mathrm{~min}$, then $\mathrm{rt}, 2 \mathrm{~h}$

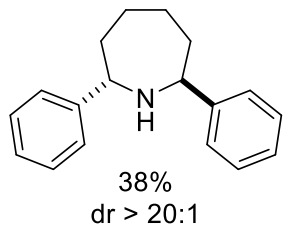

a. $n$-BuLi ( 1 equiv), $-78^{\circ} \mathrm{C}, 10 \mathrm{~min}$

b. Ketone 1 (1.05 equiv), $-78^{\circ} \mathrm{C}, 10 \mathrm{~min}$

c. PhLi (1.2 equiv), $-78^{\circ} \mathrm{C}, 10 \mathrm{~min}$, then $\mathrm{rt}, 2 \mathrm{~h}$

d. Ketone 1 ( 1.2 equiv), $-78^{\circ} \mathrm{C}, 10 \mathrm{~min}$

e. PhLi ( 1.5 equiv), $-78^{\circ} \mathrm{C}, 10 \mathrm{~min}$, then $\mathrm{rt}, 2 \mathrm{~h}$

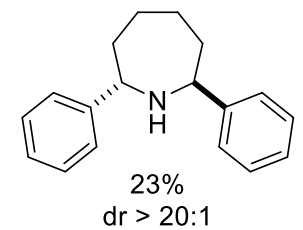

Ketone 1<smiles>O=C(c1ccccc1)C(F)(F)F</smiles>

Ketone 2<smiles>CC(C)(C)C(=O)c1ccccc1</smiles>

${ }^{\text {a }}$ Reactions were performed with $1 \mathrm{mmol}$ of the amine. Yields correspond to isolated product. 


\title{
Evaluation of Reaction Conditions for the $\alpha, \alpha^{\prime}$-Difunctionalization of Azocane. ${ }^{\text {a }}$
}

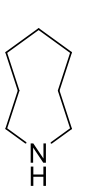
a. $n$-BuLi ( 1 equiv), $-78^{\circ} \mathrm{C}, 10 \mathrm{~min}$
b. Ketone 1 (1.05 equiv), $-78^{\circ} \mathrm{C}, 10 \mathrm{~min}$
c. PhLi ( 1.2 equiv), $-78^{\circ} \mathrm{C}, 2 \mathrm{~h}$
d. Ketone 2 ( 1.2 equiv), $-78^{\circ} \mathrm{C}, 10 \mathrm{~min}$
e. $\mathrm{PhLi}\left(1.5\right.$ equiv), $-78^{\circ} \mathrm{C}, 10 \mathrm{~min}$, then rt, $2 \mathrm{~h}$
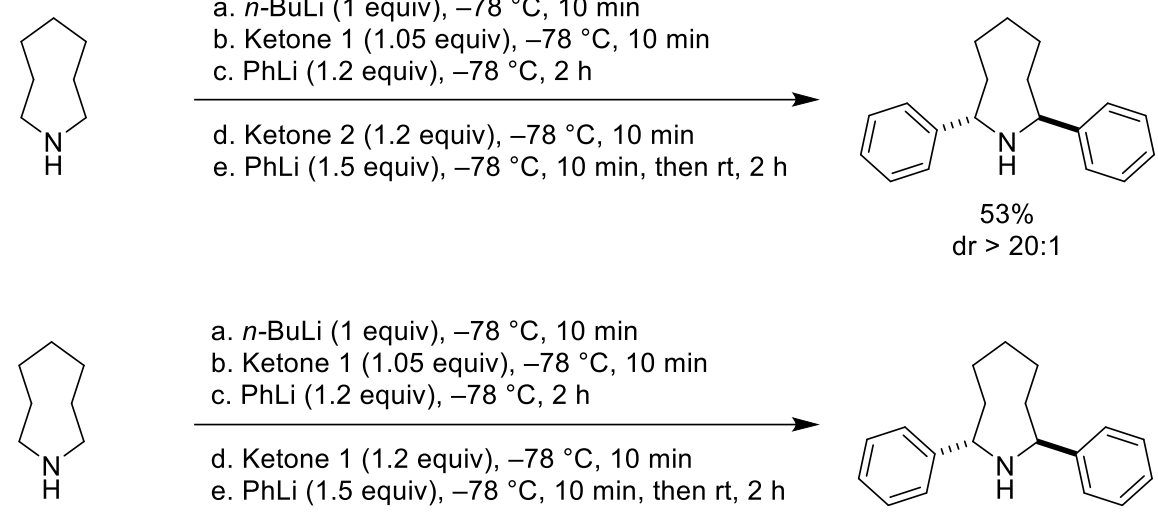

\begin{abstract}
a. $n$-BuLi ( 1 equiv), $-78{ }^{\circ} \mathrm{C}, 10 \mathrm{~min}$
b. Ketone 1 (1.05 equiv), $-78^{\circ} \mathrm{C}, 10 \mathrm{~min}$

c. PhLi (1.2 equiv), $-78^{\circ} \mathrm{C}, 2 \mathrm{~h}$

d. Ketone 1 (1.2 equiv), $-78^{\circ} \mathrm{C}, 10 \mathrm{~min}$

e. PhLi ( 1.5 equiv), $-78^{\circ} \mathrm{C}, 10 \mathrm{~min}$, then $\mathrm{rt}, 2 \mathrm{~h}$
\end{abstract}
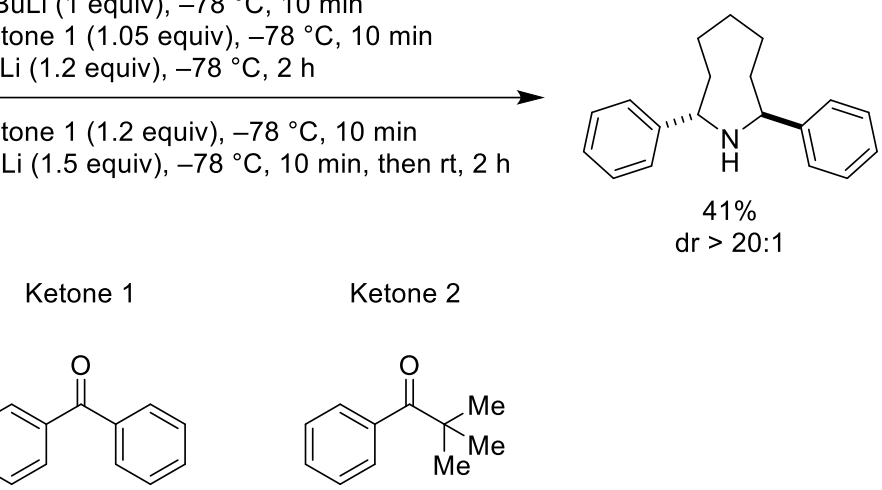

Ketone 2

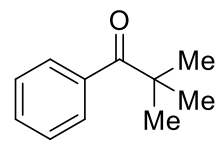

${ }^{\text {a }}$ Reactions were performed with $1 \mathrm{mmol}$ of the amine. Yields correspond to isolated product. 


\section{General Procedure A for the $\alpha, \alpha^{\prime}-\mathrm{C}-\mathrm{H}$ Bond Functionalization of Secondary Alicyclic Amines:}

To a stirred solution of the alicyclic amine $\left(1 \mathrm{mmol}, 1\right.$ equiv) in dry ether $(2 \mathrm{~mL})$ cooled to $-78{ }^{\circ} \mathrm{C}$ (dry ice-acetone bath) was added dropwise $n$-butyl lithium solution in hexanes $(1 \mathrm{mmol}, 1$ equiv) under nitrogen. The mixture was stirred at $-78^{\circ} \mathrm{C}$ for $10 \mathrm{~min}$, followed by the addition of the first ketone oxidant in dry ether $(1 \mathrm{~mL})$ via cannula. The mixture was stirred at $-78{ }^{\circ} \mathrm{C}$ for 10 minutes, followed by the dropwise addition of the first nucleophile. The mixture was stirred at $-78{ }^{\circ} \mathrm{C}$ for 2 hours. The second ketone oxidant in dry ether $(1 \mathrm{~mL})$ was then added via cannula. The mixture was stirred at $-78{ }^{\circ} \mathrm{C}$ for $10 \mathrm{~min}$, followed by addition of the second nucleophile. The mixture was allowed to warm to room temperature and stirred for 2 hours, before being cooled back to $-78{ }^{\circ} \mathrm{C}$ (dry ice-acetone bath) and quenched with methanol $(1 \mathrm{~mL})$. The mixture was allowed to warm to room temperature before adding water $(20 \mathrm{~mL})$. The resulting mixture was extracted with ethyl acetate $(3 \times 15 \mathrm{~mL})$ and dried over anhydrous $\mathrm{Na}_{2} \mathrm{SO}_{4}$. Solvent was then removed under reduced pressure and the residue purified by silica gel chromatography.

\section{General Procedure B for the $\alpha, \alpha^{\prime}-\mathrm{C}-\mathrm{H}$ Bond Functionalization of Secondary Alicyclic Amines:}

To a stirred solution of the alicyclic amine $\left(1 \mathrm{mmol}, 1\right.$ equiv) in dry ether $(2 \mathrm{~mL})$ cooled to $-78{ }^{\circ} \mathrm{C}$ (dry ice-acetone bath) was added dropwise $n$-butyl lithium solution in hexanes ( $1 \mathrm{mmol}, 1$ equiv) under nitrogen. The mixture was stirred at $-78^{\circ} \mathrm{C}$ for $10 \mathrm{~min}$, followed by the addition of the first ketone oxidant in dry ether $(1 \mathrm{~mL})$ via cannula. The mixture was stirred at $-78{ }^{\circ} \mathrm{C}$ for 10 minutes, followed by the dropwise addition of the first nucleophile. The mixture was stirred at $-78{ }^{\circ} \mathrm{C}$ for 2 hours. The second ketone oxidant in dry ether $(1 \mathrm{~mL})$ was then added via cannula. The mixture was stirred at $-78{ }^{\circ} \mathrm{C}$ for $10 \mathrm{~min}$, followed by addition of the second nucleophile. The mixture was stirred at $-78{ }^{\circ} \mathrm{C}$ for 2 hours and then quenched with methanol $(1 \mathrm{~mL})$. The mixture was allowed to warm to room temperature before adding water $(20 \mathrm{~mL})$. The resulting mixture was extracted with ethyl acetate $(3 \mathrm{x}$ $15 \mathrm{~mL}$ ) and dried over anhydrous $\mathrm{Na}_{2} \mathrm{SO}_{4}$. Solvent was then removed under reduced pressure and the residue purified by silica gel chromatography.

Note: In cases where more than one product diastereomer was obtained, dr values shown are based on the corresponding amounts of the two individual diastereomers obtained after separation via a single silica gel column chromatographic purification under the conditions provided. Both diastereomers were fully characterized for all products with drs lower than 5:1. 
$\left(2 S^{*}, 5 S^{*}\right)$-2,5-Diphenylpyrrolidine

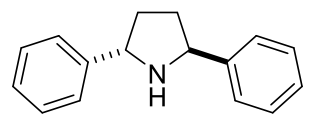

(土)-1a

Following general procedure A, compound ( \pm )-1a was obtained from pyrrolidine ( $82 \mu \mathrm{L}, 1 \mathrm{mmol})$, benzophenone (191.3 mg, $1.05 \mathrm{mmol}$ ), phenyl lithium (1.2 mmol) (first substitution), $t$-butyl phenyl ketone (201 $\mu \mathrm{L}, 1.2 \mathrm{mmol})$, and phenyl lithium (1.5 mmol) (second substitution), as a yellow oil in $48 \%$ yield (106.9 $\mathrm{mg}$ ). Dichloromethane followed by hexanes containing ethyl acetate (10-30\%) was used as the eluent for silica gel chromatography. Compound ( \pm -1a is known and the published characterization data matched our own in all respects. ${ }^{3}$ 


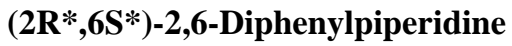

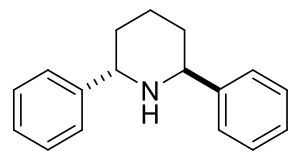

$( \pm)-1 b$

Following general procedure A, compound $( \pm)-\mathbf{1 b}$ was obtained from piperidine (99 $\mu \mathrm{L}, 1 \mathrm{mmol}$ ), trifluoroacetophenone $(147 \mu \mathrm{L}, 1.05 \mathrm{mmol})$, phenyl lithium $(1.2 \mathrm{mmol})$ (first substitution), trifluoroacetophenone ( $239 \mu \mathrm{L}, 1.7 \mathrm{mmol})$, and phenyl lithium $(2 \mathrm{mmol})$ (second substitution), as colorless oil in $70 \%$ yield $(165.7 \mathrm{mg})$. Dichloromethane followed by hexanes containing ethyl acetate (10-30\%) was used as the eluent for silica gel chromatography. Compound $( \pm)-\mathbf{1 b}$ is known and the published characterization data matched our own in all respects. $^{3}$ 


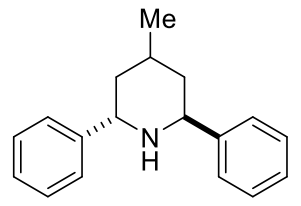

$( \pm)-1 \mathrm{c}$

Following general procedure A, compound ( \pm )-1c was obtained from 4-methylpiperidine (118 $\mu \mathrm{L}, 1 \mathrm{mmol})$, trifluoroacetophenone $(147 \mu \mathrm{L}, 1.05 \mathrm{mmol})$, phenyl lithium (1.2 mmol) (first substitution), trifluoroacetophenone $(239 \mu \mathrm{L}, 1.7 \mathrm{mmol})$, and phenyl lithium ( $2 \mathrm{mmol}$ ) (second substitution), as a colorless oil in $33 \%$ yield (83 mg) and 10:1 diastereomeric ratio. Dichloromethane followed by hexanes containing ethyl acetate (2-20\%) was used as the eluent for silica gel chromatography.

\section{Characterization data for $( \pm)-1 c$ :}

$\mathbf{R}_{\mathbf{f}}=0.47$ in hexanes/EtOAc $80: 20 \mathrm{v} / \mathrm{v}$.

${ }^{1}$ H-NMR $\left(300 \mathrm{MHz}, \mathrm{CDCl}_{3}\right): \delta=7.53-7.33(\mathrm{comp}, 8 \mathrm{H}), 7.32-7.23(\mathrm{comp}, 2 \mathrm{H}), 4.63-4.38(\mathrm{~m}, 1 \mathrm{H}), 3.88(\mathrm{dd}, J=$ 11.6, $2.5 \mathrm{~Hz}, 1 \mathrm{H}), 2.62-2.14$ (comp, 2H), 1.97-1.61 (comp, 3H), 1.31 (app dt, $J=13.1,11.4 \mathrm{~Hz}, 1 \mathrm{H}), 1.01(\mathrm{~d}, J=$ $5.9 \mathrm{~Hz}, 3 \mathrm{H})$.

${ }^{13}$ C-NMR $\left(75 \mathrm{MHz}, \mathrm{CDCl}_{3}\right): \delta=145.3,143.3,128.6,128.5,127.2,126.8,126.7,126.2,54.9,54.8,43.5,36.9,26.9$, 22.8 .

HRMS (ESI-TOF): Calculated for $\mathrm{C}_{18} \mathrm{H}_{22} \mathrm{~N}[\mathrm{M}+\mathrm{H}]^{+}: 252.1747$, found: 252.1739 . 


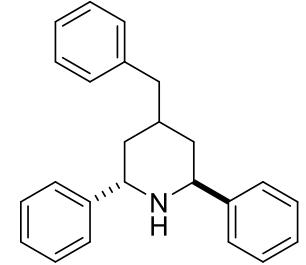

$( \pm)-1 d$

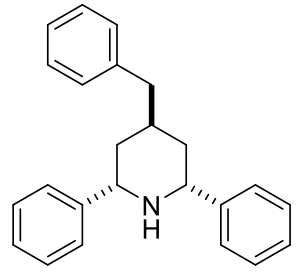

$( \pm)-1 d^{\prime}$

Following general procedure A, compound $( \pm)$-1d and $( \pm)$-1d' was obtained from 4-benzylpiperidine (176 $\mu \mathrm{L}, 1$ mmol), trifluoroacetophenone (147 $\mu \mathrm{L}, 1.05 \mathrm{mmol})$, phenyl lithium $(1.2 \mathrm{mmol})$ (first substitution), trifluoroacetophenone $(239 \mu \mathrm{L}, 1.7 \mathrm{mmol})$, and phenyl lithium $(2 \mathrm{mmol})$ (second substitution), in $48 \%$ combined yield $(157.2 \mathrm{mg}$ ) and 3.8:1 diastereomeric ratio. Dichloromethane followed by hexanes containing ether (2-20\%) was used as the eluent for silica gel chromatography.

Characterization data for major diastereomer ( \pm )-1d: a colorless oil in 38\% yield (124.5 mg)

$\mathbf{R}_{\mathbf{f}}=0.24$ in hexanes/Et $2 \mathrm{O} 70: 30 \mathrm{v} / \mathrm{v}$.

${ }^{1}$ H-NMR (400 MHz, $\left.\mathrm{CDCl}_{3}\right): \delta=7.44-7.42(\mathrm{~m}, 1 \mathrm{H}), 7.42-7.39$ (comp, 5H), 7.39-7.34 (comp, 2H), 7.34-7.26 (comp, 4H), 7.26-7.17 (comp, 3H), 4.52-4.49 (m, 1H), 3.84 (dd, $J=11.5,2.6 \mathrm{~Hz}, 1 \mathrm{H}), 2.67$ (dd, $J=13.4,7.0 \mathrm{~Hz}$, $1 \mathrm{H}), 2.61(\mathrm{dd}, J=13.4,7.3 \mathrm{~Hz}, 1 \mathrm{H}), 2.47-2.40(\mathrm{~m}, 1 \mathrm{H}), 2.29$ (br s, $1 \mathrm{H}), 2.06$ (app tdt, $J=15.5,7.0,3.5 \mathrm{~Hz}, 1 \mathrm{H}$ ), $1.88-1.78$ (comp, 2H), 1.39 (app q, $J=11.9 \mathrm{~Hz}, 1 \mathrm{H}$ ).

${ }^{13}$ C-NMR $\left(100 \mathrm{MHz}, \mathrm{CDCl}_{3}\right): \delta=145.0,142.8,140.1,129.1,128.5,128.4,128.2,127.1,126.7,126.5,126.1,125.9$, $54.8,54.6,43.8,41.1,34.6,33.7$.

HRMS (ESI-TOF): Calculated for $\mathrm{C}_{24} \mathrm{H}_{26} \mathrm{~N}[\mathrm{M}+\mathrm{H}]^{+}: 328.2060$, found: 328.2044 . 
Characterization data for minor diastereomer ( \pm )-1d': a colorless oil in 10\% yield (32 mg)

$\mathbf{R}_{\mathbf{f}}=0.60$ in hexanes/ $\mathrm{Et}_{2} \mathrm{O} 70: 30 \mathrm{v} / \mathrm{v}$.

${ }^{1} \mathbf{H}-\mathbf{N M R}\left(400 \mathrm{MHz}, \mathrm{CDCl}_{3}\right.$ ): $\delta=7.76-7.70$ (comp, 4H), 7.61-7.55 (comp, 6H), 7.54-7.44 (comp, 5H), 4.48 (dd, $J$ $=11.1,3.3 \mathrm{~Hz}, 2 \mathrm{H}), 3.27(\operatorname{app~d}, J=8.1 \mathrm{~Hz}, 2 \mathrm{H}), 2.66-2.57(\mathrm{~m}, 1 \mathrm{H}), 2.31$ (br s, 1H), 2.09-1.93 (comp, 4H).

${ }^{13} \mathrm{C}-\mathrm{NMR}\left(100 \mathrm{MHz}, \mathrm{CDCl}_{3}\right): \delta=145.5,141.2,129.0,128.4,128.3,127.0,126.7,125.9,56.7,38.1,37.9,35.3$.

HRMS (ESI-TOF): Calculated for $\mathrm{C}_{24} \mathrm{H}_{26} \mathrm{~N}[\mathrm{M}+\mathrm{H}]^{+}:$328.2060, found: 328.2041 . 


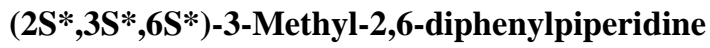

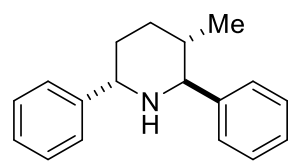

$( \pm)-1 e$

Following general procedure A, compound $( \pm)$-1e was obtained from 3-methylpiperidine $(117.4 \mu \mathrm{L}, 1 \mathrm{mmol})$, trifluoroacetophenone $(147 \mu \mathrm{L}, 1.05 \mathrm{mmol})$, phenyl lithium $(1.2 \mathrm{mmol})$ (first substitution), trifluoroacetophenone ( $239 \mu \mathrm{L}, 1.7 \mathrm{mmol}$ ), and phenyl lithium ( $2 \mathrm{mmol}$ ) (second substitution), as a colorless oil in $45 \%$ yield (113 $\mathrm{mg}$ ). Dichloromethane followed by hexanes containing ether (10-30\%) was used as the eluent for silica gel chromatography.

\section{Characterization data for $( \pm)-1 \mathrm{e}$ :}

$\mathbf{R}_{\mathbf{f}}=0.18$ in hexanes $/ \mathrm{Et}_{2} \mathrm{O} 70: 30 \mathrm{v} / \mathrm{v}$.

${ }^{1} \mathbf{H}-N M R\left(400 \mathrm{MHz}, \mathrm{CDCl}_{3}\right.$ ): $\delta=7.50-7.47$ (comp, 2H), 7.46-7.36 (comp, 6H), 7.34-7.28 (comp, 2H), 4.38 (app t, $J=3.8 \mathrm{~Hz}, 1 \mathrm{H}), 3.47$ (d, $J=9.3 \mathrm{~Hz}, 1 \mathrm{H}), 2.41$ (app dtd, $J=13.7,3.8,2.8 \mathrm{~Hz}, 1 \mathrm{H}), 2.32-2.18(\mathrm{comp}, 2 \mathrm{H}), 1.96-1.84$ $(\mathrm{m}, 1 \mathrm{H}), 1.79($ app dq, $J=13.5,3.9 \mathrm{~Hz}, 1 \mathrm{H}), 1.37($ app tdd, $J=13.1,11.0,3.7 \mathrm{~Hz}, 1 \mathrm{H}), 0.69(\mathrm{~d}, J=6.6 \mathrm{~Hz}, 3 \mathrm{H})$.

${ }^{13}$ C-NMR $\left(100 \mathrm{MHz}, \mathrm{CDCl}_{3}\right): \delta=143.8,143.2,128.5,128.3,127.7,127.2,126.6,126.1,62.4,54.5,37.0,29.2,28.8$, 18.8 .

HRMS (ESI-TOF): Calculated for $\mathrm{C}_{18} \mathrm{H}_{22} \mathrm{~N}[\mathrm{M}+\mathrm{H}]^{+}: 252.1747$, found: 252.1752 . 


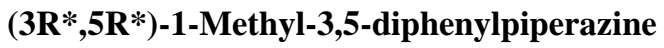

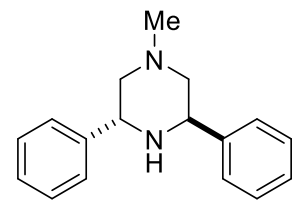

(士)-1f

Following general procedure A, compound ( \pm )-1f was obtained from 1-methylpiperazine (111 $\mu \mathrm{L}, 1 \mathrm{mmol})$, trifluoroacetophenone $(190 \mu \mathrm{L}, 1.35 \mathrm{mmol})$, phenyl lithium (1.5 mmol) (first substitution), trifluoroacetophenone (239 $\mu \mathrm{L}, 1.7 \mathrm{mmol}$ ), and phenyl lithium (2 mmol) (second substitution), as a colorless oil in $39 \%$ yield (97.8 mg). Dichloromethane followed by hexanes containing ethyl acetate (10-30\%) was used as the eluent for silica gel chromatography.

\section{Characterization data for $( \pm)-1 f$ :}

$\mathbf{R}_{\mathbf{f}}=0.46$ in hexanes/EtOAc $70: 30 \mathrm{v} / \mathrm{v}$.

${ }^{1} \mathbf{H}-\mathbf{N M R}\left(500 \mathrm{MHz}, \mathrm{CDCl}_{3}\right): \delta=7.53-7.50$ (comp, 4H), 7.36-7.32 (comp, 4H), 7.28-7.23 (comp, $\left.2 \mathrm{H}\right), 4.16$ (app t, $J=4.8 \mathrm{~Hz}, 2 \mathrm{H}), 2.77-2.70$ (comp, 4H), 2.33 (s, 3H).

${ }^{13}$ C-NMR $\left(125 \mathrm{MHz}, \mathrm{CDCl}_{3}\right): \delta=142.7,128.3,127.3,127.0,60.3,54.5,46.5$.

HRMS (ESI-TOF): Calculated for $\mathrm{C}_{17} \mathrm{H}_{21} \mathrm{~N}_{2}[\mathrm{M}+\mathrm{H}]^{+}:$253.1699, found: 253.1696 . 


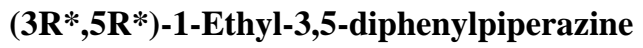

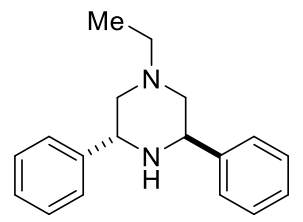

$( \pm)-19$

Following general procedure A, compound ( \pm )-1g was obtained from 1-ethylpiperazine (127 $\mu \mathrm{L}, 1 \mathrm{mmol})$, trifluoroacetophenone $(147 \mu \mathrm{L}, 1.05 \mathrm{mmol})$, phenyl lithium (1.2 mmol) (first substitution), trifluoroacetophenone $(239 \mu \mathrm{L}, 1.7 \mathrm{mmol})$, and phenyl lithium (2 mmol) (second substitution), as a yellow oil in $45 \%$ yield (119.8 mg). Dichloromethane followed by hexanes containing ethyl acetate (5-20\%) was used as the eluent for silica gel chromatography.

\section{Characterization data for ( \pm )-1g:}

$\mathbf{R}_{\mathbf{f}}=0.45$ in hexanes/EtOAc $70: 30 \mathrm{v} / \mathrm{v}$.

${ }^{1} \mathbf{H}-\mathbf{N M R}\left(400 \mathrm{MHz}, \mathrm{CDCl}_{3}\right): \delta=7.56-7.52$ (comp, 4H), 7.37-7.32 (comp, 4H), 7.29-7.24 (comp, $\left.2 \mathrm{H}\right), 4.16$ (app t, $J=4.8 \mathrm{~Hz}, 2 \mathrm{H}), 2.85-2.76(\mathrm{comp}, 4 \mathrm{H}), 2.53(\mathrm{dq}, J=12.2,7.3 \mathrm{~Hz}, 1 \mathrm{H}), 2.42(\mathrm{dq}, J=12.2,7.1 \mathrm{~Hz}, 1 \mathrm{H}), 2.23(\mathrm{br}$ s, $1 \mathrm{H}), 1.17(\mathrm{t}, J=7.2 \mathrm{~Hz}, 3 \mathrm{H})$.

${ }^{13}$ C-NMR $\left(100 \mathrm{MHz}, \mathrm{CDCl}_{3}\right): \delta=143.0,128.3,127.5,127.0,57.9,54.6,52.6,12.0$.

HRMS (ESI-TOF): Calculated for $\mathrm{C}_{18} \mathrm{H}_{23} \mathrm{~N}_{2}[\mathrm{M}+\mathrm{H}]^{+}:$267.1856, found: 267.1866. 


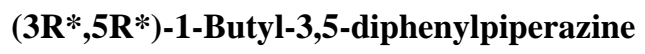

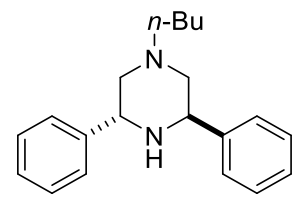

$( \pm)-1 h$

Following general procedure A, compound $( \pm)-\mathbf{1 h}$ was obtained from 1-butylpiperazine $(142.2 \mathrm{mg}, 1 \mathrm{mmol})$, trifluoroacetophenone $(147 \mu \mathrm{L}, 1.05 \mathrm{mmol})$, phenyl lithium (1.2 mmol) (first substitution), trifluoroacetophenone $(239 \mu \mathrm{L}, 1.7 \mathrm{mmol})$, and phenyl lithium (2 mmol) (second substitution), as a yellow oil in $48 \%$ yield (141.3 mg). Dichloromethane followed by hexanes containing ethyl acetate (5-20\%) was used as the eluent for silica gel chromatography.

\section{Characterization data for $( \pm)-1 \mathrm{~h}$ :}

$\mathbf{R}_{\mathbf{f}}=0.55$ in hexanes/EtOAc $70: 30 \mathrm{v} / \mathrm{v}$.

${ }^{1} \mathbf{H}-\mathbf{N M R}\left(400 \mathrm{MHz}, \mathrm{CDCl}_{3}\right): \delta=7.58-7.54$ (comp, 4H), 7.38-7.33 (comp, 4H), 7.30-7.25 (comp, 2H), 4.16 (app t, $J=4.8 \mathrm{~Hz}, 2 \mathrm{H}), 2.85-2.74$ (comp, 4H), 2.47-2.32 (comp, 2H), 2.19 (br s, 1H), 1.67-1.49 (comp, 2H), 1.49-1.39 (comp, 2H), $0.98(\mathrm{t}, J=7.3 \mathrm{~Hz}, 3 \mathrm{H})$.

${ }^{13}$ C-NMR $\left(100 \mathrm{MHz}, \mathrm{CDCl}_{3}\right): \delta=143.1,128.2,127.5,126.9,58.7,58.5,54.7,28.9,20.7,14.0$.

HRMS (ESI-TOF): Calculated for $\mathrm{C}_{20} \mathrm{H}_{27} \mathrm{~N}_{2}[\mathrm{M}+\mathrm{H}]^{+}:$295.2169, found: 295.2181. 


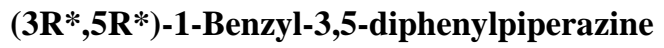

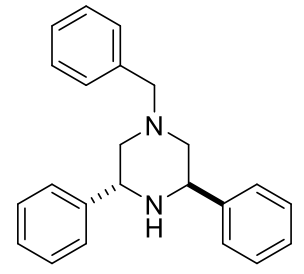

(士)-1i

Following general procedure A, compound ( \pm )-1i was obtained from 1-benzylpiperazine (174 $\mu \mathrm{L}, 1 \mathrm{mmol})$, trifluoroacetophenone $(147 \mu \mathrm{L}, 1.05 \mathrm{mmol})$, phenyl lithium (1.2 mmol) (first substitution), trifluoroacetophenone $(239 \mu \mathrm{L}, 1.7 \mathrm{mmol})$, and phenyl lithium (2 mmol) (second substitution), as a pale yellow oil in $47 \%$ yield (155.4

$\mathrm{mg}$ ). Dichloromethane followed by hexanes containing ethyl acetate (10-30\%) was used as the eluent for silica gel chromatography.

\section{Characterization data $( \pm)-1 \mathrm{i}$ :}

$\mathbf{R}_{\mathbf{f}}=0.39$ in hexanes/EtOAc $80: 20 \mathrm{v} / \mathrm{v}$.

${ }^{1} \mathbf{H}-\mathbf{N M R}\left(300 \mathrm{MHz}, \mathrm{CDCl}_{3}\right): \delta=7.56(\operatorname{app~d}, J=7.3 \mathrm{~Hz}, 4 \mathrm{H}), 7.50-7.23(\mathrm{comp}, 11 \mathrm{H}), 4.19$ (dd, $J=5.6,3.8 \mathrm{~Hz}$, 2H), 3.63 (comp 2H), 2.91-2.79 (comp, 4H), 2.55 (br s, 1H).

${ }^{13} \mathrm{C}-\mathrm{NMR}\left(75 \mathrm{MHz}, \mathrm{CDCl}_{3}\right): \delta=142.9,138.0,129.1,128.4,128.2,127.5,127.0,126.9,63.4,58.1,54.6$.

HRMS (ESI-TOF): Calculated for $\mathrm{C}_{23} \mathrm{H}_{25} \mathrm{~N}_{2}[\mathrm{M}+\mathrm{H}]^{+}:$329.2012, found: 329.1995 . 


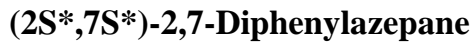

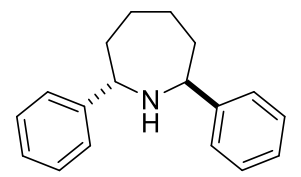

$( \pm)-1 \mathbf{j}$

Following general procedure A, compound $( \pm)-\mathbf{1 j}$ was obtained from azepane $(113 \mu \mathrm{L}, 1 \mathrm{mmol})$, trifluoroacetophenone $(147 \mu \mathrm{L}, 1.05 \mathrm{mmol})$, phenyl lithium $(1.2 \mathrm{mmol})$ (first substitution), $t$-butyl phenyl ketone $(201 \mu \mathrm{L}, 1.2 \mathrm{mmol})$ and phenyl lithium $(1.5 \mathrm{mmol})$ for the second arylation, as a yellow oil in $53 \%$ yield $(132.2 \mathrm{mg})$. Dichloromethane followed by hexanes containing ethyl acetate (10-30\%) was used as the eluent for silica gel chromatography. Compound $( \pm)-\mathbf{1 j}$ is known and the published characterization data matched our own in all respects. $^{3}$ 


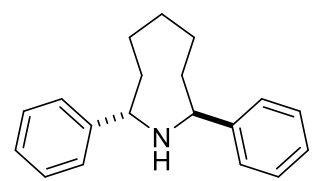

$$
( \pm)-1 k
$$

Following general procedure A, compound $( \pm)-\mathbf{1 k}$ was obtained from azocane $(126 \mu \mathrm{L}, 1 \mathrm{mmol})$, benzophenone (191.33 mg, $1.05 \mathrm{mmol})$, phenyl lithium (1.2 mmol) (first substitution), $t$-butyl phenyl ketone $(201 \mu \mathrm{L}, 1.2 \mathrm{mmol})$, and phenyl lithium $(1.5 \mathrm{mmol})$ (second substitution), as a white solid in 53\% yield (140.6 mg). Dichloromethane followed by hexanes containing ethyl acetate (5-25\%) was used as the eluent for silica gel chromatography.

\section{Characterization data for $( \pm)-1 \mathrm{k}$ :}

$\mathbf{R}_{\mathbf{f}}=0.37$ in hexanes $/$ EtOAc $80: 20 \mathrm{v} / \mathrm{v}$

m.p.: $67-70{ }^{\circ} \mathrm{C}$

${ }^{1} \mathrm{H}-\mathrm{NMR}\left(300 \mathrm{MHz}, \mathrm{CDCl}_{3}\right): \delta=7.32-7.13$ (comp, 10H), 4.09 (dd, $\left.J=9.8,3.5 \mathrm{~Hz}, 2 \mathrm{H}\right), 2.11-1.68$ (comp, 11H).

${ }^{13}$ C-NMR $\left(75 \mathrm{MHz}, \mathrm{CDCl}_{3}\right): \delta=146.8,128.2,126.6,126.3,58.0,34.4,27.8,25.7$.

HRMS (ESI-TOF): Calculated for $\mathrm{C}_{19} \mathrm{H}_{23} \mathrm{~N}[\mathrm{M}+\mathrm{H}]^{+}: 266.1903$, found: 266.1927 . 
$\left(2 R^{*}, 13 S^{*}\right)$-2,13-Diphenylazacyclotridecane and $\left(2 S^{*}, 13 S^{*}\right)$-2,13-Diphenylazacyclotridecane

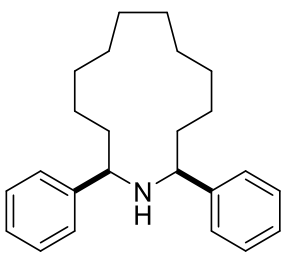

$( \pm)-11$

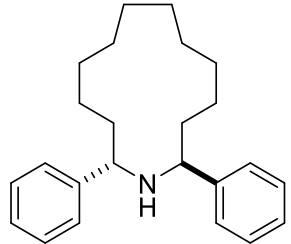

$( \pm)-1 I^{\prime}$

Following general procedure A, compound ( \pm )-11 and ( \pm )-11' was obtained from azacyclotridecane (183 mg, 1 $\mathrm{mmol})$, trifluoroacetophenone (147 $\mu \mathrm{L}, 1.05 \mathrm{mmol})$, phenyl lithium $(1.2 \mathrm{mmol})$ (first substitution), trifluoroacetophenone $(239 \mu \mathrm{L}, 1.7 \mathrm{mmol})$, and phenyl lithium $(2 \mathrm{mmol})$ (second substitution), in a combined $45 \%$ yield $(151 \mathrm{mg})$ and 3:1 diastereomeric ratio. Dichloromethane followed by hexanes containing ether (2-20\%) was used as the eluent for silica gel chromatography.

Characterization data for major diastereomer ( \pm )-11: a white solid in $34 \%$ yield (113 mg)

$\mathbf{R}_{\mathbf{f}}=0.78$ in hexanes $/ \mathrm{Et}_{2} \mathrm{O} 80: 20 \mathrm{v} / \mathrm{v}$.

m.p.: $64-66^{\circ} \mathrm{C}$

${ }^{1} \mathbf{H}-\mathrm{NMR}$ (400 MHz, $\mathrm{CDCl}_{3}$ ): $\delta=7.29-7.17$ (comp, 10H), 3.78-3.73 (comp, 2H), 1.79-1.67 (comp, 6H), 1.61-1.39 (comp, 15H).

${ }^{13}$ C-NMR (100 MHz, $\left.\mathrm{CDCl}_{3}\right): \delta=146.1,128.0,126.7,126.3,60.1,37.7,26.5,26.3,24.7,23.1$.

HRMS (ESI-TOF): Calculated for $\mathrm{C}_{24} \mathrm{H}_{34} \mathrm{~N}[\mathrm{M}+\mathrm{H}]^{+}:$336.2686, found: 336.2667 .

The title compound was further characterized by X-ray crystallography.

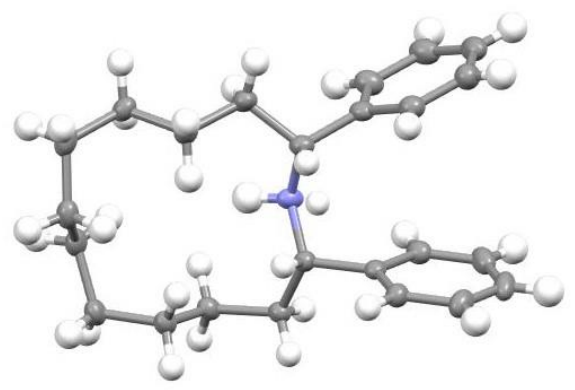

Thermal ellipsoids are drawn at the 50\% probability level.

Compound $\mathbf{1 l}$ was crystallized from $\mathrm{CH}_{2} \mathrm{Cl}_{2} /$ hexane through vapor diffusion at room temperature. The requisite $\mathrm{CIF}$ has been deposited with the CCDC (deposition \# 2039636). 
Characterization data for minor diastereomer ( \pm )-11': a colorless oil in 11\% yield (38 mg)

$\mathbf{R}_{\mathbf{f}}=0.60$ in hexanes/ $\mathrm{Et}_{2} \mathrm{O} 70: 30 \mathrm{v} / \mathrm{v}$.

${ }^{1} \mathbf{H}-\mathbf{N M R}\left(400 \mathrm{MHz}, \mathrm{CDCl}_{3}\right): \delta=7.33-7.28$ (comp, 4H), 7.26-7.20 (comp, 6H), 3.81 (app t, $\left.J=6.1 \mathrm{~Hz}, 2 \mathrm{H}\right), 1.96-$ 1.85 (comp, 2H), 1.78-1.68 (comp, 2H), 1.61-1.36 (comp, 17H).

${ }^{13}$ C-NMR $\left(100 \mathrm{MHz} \mathrm{CDCl}_{3}\right): \delta=143.7,128.2,127.4,126.6,58.8,34.5,27.0,26.2,24.9,23.5$.

HRMS (ESI-TOF): Calculated for $\mathrm{C}_{24} \mathrm{H}_{34} \mathrm{~N}[\mathrm{M}+\mathrm{H}]^{+}:$336.2686, found: 336.2698. 
$(1 \mathrm{R} *, 3 \mathrm{~S} *, 3 \mathrm{aS} *, 6 \mathrm{aR} *)-1,3-\mathrm{Diphenyloctahydrocyclopenta[c]pyrrole}$

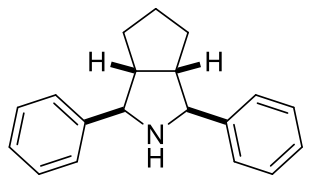

$( \pm)-1 \mathrm{~m}$

Following general procedure A, compound $( \pm)-\mathbf{1 m}$ was obtained from octahydrocyclopenta[c]pyrrole (111 mg, 1 mmol), trifluoroacetophenone $(147 \mu \mathrm{L}, 1.05 \mathrm{mmol})$, phenyl lithium $(1.2 \mathrm{mmol})$ (first substitution), trifluoroacetophenone ( $239 \mu \mathrm{L}, 1.7 \mathrm{mmol}$ ), and phenyl lithium ( $2 \mathrm{mmol}$ ) (second substitution), as a white solid in $45 \%$ yield $(118.5 \mathrm{mg})$. Dichloromethane followed by hexanes containing ether $(2-8 \%)$ was used as the eluent for silica gel chromatography.

Characterization data for $( \pm)-1 \mathrm{~m}$ :

$\mathbf{R}_{\mathbf{f}}=0.65$ in hexanes/Et $2 \mathrm{O} 80: 20 \mathrm{v} / \mathrm{v}$.

m.p.: $106-107^{\circ} \mathrm{C}$

${ }^{1} \mathbf{H}-\mathrm{NMR}$ (400 MHz, $\mathrm{CDCl}_{3}$ ): $\delta=7.61-7.55$ (comp, 4H), 7.42-7.35 (comp, 4H), 7.33-7.26 (comp, 2H), 3.82-3.73 (comp, 2H), 2.63-2.53 (comp, 2H), 2.09 (br s, 1H), 1.83-1.71 (comp, 4H), 1.63-1.51 (comp, 2H).

${ }^{13}$ C-NMR (100 MHz, $\left.\mathrm{CDCl}_{3}\right): \delta=144.5,128.3,127.0,126.9,69.4,53.5,30.7,24.8$.

HRMS (ESI-TOF): Calculated for $\mathrm{C}_{19} \mathrm{H}_{22} \mathrm{~N}[\mathrm{M}+\mathrm{H}]^{+}: 264.1747$, found: 264.1722 .

The title compound was further characterized by X-ray crystallography:

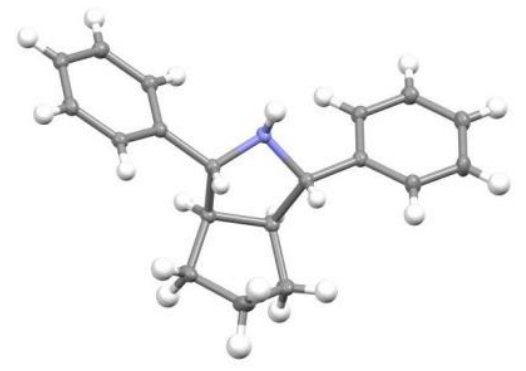

Thermal ellipsoids are drawn at the 50\% probability level.

Compound 1m was crystallized from THF through slow evaporation at room temperature. The requisite CIF has been deposited with the CCDC (deposition \# 2039637). 
$\left(1 \mathrm{R}^{*}, 3 \mathrm{~S} *, 3 \mathrm{aS} *, 4 \mathrm{R}^{*}, 7 \mathrm{~S}^{*}, 7 \mathrm{aR} *\right)$-1,3-Diphenyl-2,3,3a,4,7,7a-hexahydro-1H-4,7-methanoisoindole

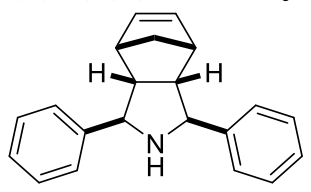

$( \pm)-1 n$

To a stirred solution of the corresponding amine hydrochloride salt $(86 \mathrm{mg}, 0.5 \mathrm{mmol}, 1$ equiv) in dry ether $(1 \mathrm{~mL})$ cooled to $-78{ }^{\circ} \mathrm{C}$ (dry ice-acetone bath) was added dropwise $n$-butyl lithium solution in hexanes ( 1 mmol, 2 equiv) under nitrogen. The mixture was stirred at the same temperature for $10 \mathrm{~min}$, followed by the addition of trifluoroacetophenone ( $74 \mu \mathrm{L}, 0.525 \mathrm{mmol}, 1.05$ equiv) in dry ether $(0.5 \mathrm{~mL})$ via cannula. The mixture was stirred at the same temperature for 10 minutes, followed by the dropwise addition of phenyl lithium ( $0.6 \mathrm{mmol}, 1.2$ equiv) for the first arylation. The mixture was stirred at the same temperature for 2 hours. Trifluoroacetophenone $(120 \mu \mathrm{L}$, $0.85 \mathrm{mmol}, 1.7$ equiv) in dry ether $(0.5 \mathrm{~mL})$ was then added via cannula. The mixture was stirred at the same temperature for $10 \mathrm{~min}$, followed by addition of phenyl lithium $(1.0 \mathrm{mmol}, 2$ equiv) for the second arylation. The mixture was allowed to warm to room temperature and stirred for 2 hours, before being cooled back to $-78{ }^{\circ} \mathrm{C}$ (dry ice-acetone bath) and quenched with methanol $(1 \mathrm{~mL})$ and adding water $(20 \mathrm{~mL})$. The resulting mixture was extracted with ethyl acetate $(3 \times 15 \mathrm{~mL})$ and dried over anhydrous $\mathrm{Na}_{2} \mathrm{SO}_{4}$. Solvent was then removed under reduced pressure and the residue purified by silica gel chromatography using dichloromethane followed by hexanes containing ethyl acetate (3-10\%) as the eluent. Compound ( \pm )-1n was obtained as a white solid in $40 \%$ yield (57.5 $\mathrm{mg})$.

\section{Characterization data for $( \pm)-1 \mathrm{n}$ :}

$\mathbf{R}_{\mathbf{f}}=0.35$ in hexanes/EtOAc $85: 15 \mathrm{v} / \mathrm{v}$.

m.p.: $106-108{ }^{\circ} \mathrm{C}$

${ }^{1}$ H-NMR (600 MHz, $\mathrm{CDCl}_{3}$ ): $\delta=7.53-7.49$ (comp, 4H), 7.40-7.35 (comp, 4H), 7.30-7.26 (comp, 2H), 6.43 (t, $J=$ $1.9 \mathrm{~Hz}, 2 \mathrm{H}), 3.98-3.92$ (comp, 2H), 2.95-2.87 (comp, 4H), 2.4 (br s, 1H), 1.80 (dt, $J=8.2,1.8 \mathrm{~Hz}, 1 \mathrm{H}), 1.61-1.56$ $(\mathrm{m}, 1 \mathrm{H})$.

${ }^{13} \mathrm{C}-\mathrm{NMR}\left(151 \mathrm{MHz}, \mathrm{CDCl}_{3}\right): \delta=144.6,137.8,128.3,126.8,126.4,64.4,57.7,54.1,44.3$.

HRMS (ESI-TOF): Calculated for $\mathrm{C}_{21} \mathrm{H}_{22} \mathrm{~N}[\mathrm{M}+\mathrm{H}]^{+}: 288.1747$, found: 288.1733 . 
$\left(2 S^{*}, 6 S^{*}\right)-2,6-D i($ naphthalen-2-yl) piperidine

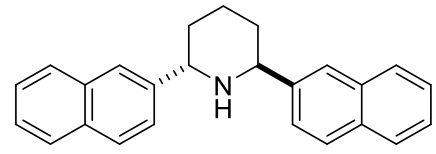

$( \pm)-10$

Following general procedure A, compound $( \pm)-\mathbf{1 0}$ was obtained from piperidine $(99 \mu \mathrm{L}, 1 \mathrm{mmol}$ ), trifluoroacetophenone $(147 \mu \mathrm{L}, 1.05 \mathrm{mmol}), 2$-bromonaphthalene $(248.5 \mathrm{mg}, 1.2 \mathrm{mmol})$ (first substitution), trifluoroacetophenone ( $239 \mu \mathrm{L}, 1.7 \mathrm{mmol})$, and 2-bromonaphthalene ( $414.1 \mathrm{mg}, 2 \mathrm{mmol}$ ) (second substitution), as a colorless oil in $60 \%$ yield $(202.5 \mathrm{mg})$. Dichloromethane followed by hexanes containing ether (5-20\%) was used as the eluent for silica gel chromatography.

\section{Characterization data for $( \pm)-10$ :}

$\mathbf{R}_{\mathbf{f}}=0.19$ in hexanes $/ \mathrm{Et}_{2} \mathrm{O} 80: 20 \mathrm{v} / \mathrm{v}$.

${ }^{1}$ H-NMR (400 MHz, $\mathrm{CDCl}_{3}$ ): $\delta=7.94-7.92$ (comp, 2H), 7.91-7.85 (comp, 6H), 7.64-7.59 (comp, 2H), 7.54-7.47 (comp, 4H), 4.36 (dd, $J=6.7,4.1 \mathrm{~Hz}, 2 \mathrm{H}), 2.56$ (br s, 1H), 2.24-2.14 (comp, 2H), 2.13-2.04 (comp, 2H), 1.90-1.82 (comp, 2H).

${ }^{13}$ C-NMR $\left(100 \mathrm{MHz}, \mathrm{CDCl}_{3}\right): \delta=141.5,133.4,132.4,128.1,127.8,127.5,126.0,125.6,125.4,125.1,54.9,31.4$, 20.8 .

HRMS (ESI-TOF): Calculated for $\mathrm{C}_{25} \mathrm{H}_{24} \mathrm{~N}[\mathrm{M}+\mathrm{H}]^{+}: 338.1903$, found: 338.1844 . 
$\left(2 S^{*}, 6 S^{*}\right)-2-P h e n y l-6-((E)$-styryl)piperidine

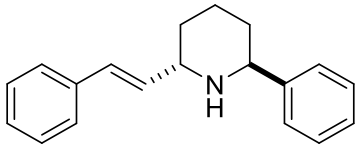

$( \pm)-1 p$

Following general procedure A, compound $( \pm)-\mathbf{1 p}$ was obtained from piperidine (99 $\mu \mathrm{L}, 1 \mathrm{mmol}$ ), trifluoroacetophenone $(147 \mu \mathrm{L}, 1.05 \mathrm{mmol})$, phenyl lithium $(1.07 \mathrm{mmol})$ (first substitution), trifluoroacetophenone ( $239 \mu \mathrm{L}, 1.7 \mathrm{mmol}$ ), and $\beta$-bromostyrene $(257 \mu \mathrm{L}, 2 \mathrm{mmol}$ ) (second substitution), as a yellow oil in $17 \%$ yield (45.1 $\mathrm{mg}$ ). Dichloromethane followed by hexanes containing ethyl acetate (5-20\%) was used as the eluent for silica gel chromatography.

\section{Characterization data for $( \pm)-1 p$ :}

$\mathbf{R}_{\mathbf{f}}=0.58$ in hexanes/EtOAc 70:30 v/v.

${ }^{1} \mathbf{H}-N M R\left(600 \mathrm{MHz}, \mathrm{CDCl}_{3}\right): \delta=7.46(\mathrm{~d}, J=7.3 \mathrm{~Hz}, 4 \mathrm{H}), 7.38$ (app td, $\left.J=7.7,3.0 \mathrm{~Hz}, 4 \mathrm{H}\right), 7.31-7.26$ (comp, 2H), $6.65(\mathrm{dd}, J=16.1,6.1 \mathrm{~Hz}, 1 \mathrm{H}), 6.57(\mathrm{~d}, J=16.1,1 \mathrm{H}), 4.15(\mathrm{dd}, J=9.5,3.2 \mathrm{~Hz}, 1 \mathrm{H}), 3.96-3.91(\mathrm{~m}, 1 \mathrm{H}), 2.66(\mathrm{br} \mathrm{s}$, 1H), 2.04-1.97 (m, 1H), 1.94-1.88 (m, 1H), 1.85-1.75 (comp, 4H).

${ }^{13}$ C-NMR $\left(151 \mathrm{MHz}, \mathrm{CDCl}_{3}\right): \delta=144.6,137.2,131.7,130.3,128.5,128.4,127.3,127.0,126.7,126.2,55.0,54.4$, $33.6,29.7,20.5$.

HRMS (ESI-TOF): Calculated for $\mathrm{C}_{19} \mathrm{H}_{22} \mathrm{~N}[\mathrm{M}+\mathrm{H}]^{+}: 264.1747$, found: 264.1711 . 
$\left(2 S^{*}, 6 S^{*}\right)-2-($ Naphthalen-2-yl)-6-phenylpiperidine

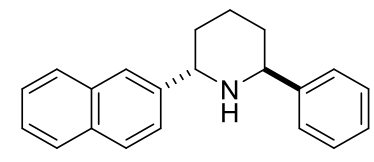

$( \pm)-1 q$

Following general procedure A, compound $( \pm)$-1q was obtained from piperidine $(99 \mu \mathrm{L}, 1 \mathrm{mmol}$ ), trifluoroacetophenone $(147 \mu \mathrm{L}, 1.05 \mathrm{mmol})$, phenyl lithium $(1.07 \mathrm{mmol})$ (first substitution), trifluoroacetophenone (239 $\mu \mathrm{L}, 1.7 \mathrm{mmol}$ ), and 2-bromonaphthalene $(414.1 \mathrm{mg}, 2 \mathrm{mmol}$ ) (second substitution), as a yellow oil in $56 \%$ yield (161 mg). Dichloromethane followed by hexanes containing ethyl acetate (10-30\%) was used as the eluent for silica gel chromatography.

\section{Characterization data for $( \pm)-1 q$ :}

$\mathbf{R}_{\mathbf{f}}=0.26$ in hexanes/EtOAc $85: 15 \mathrm{v} / \mathrm{v}$.

${ }^{1}$ H-NMR $\left(400 \mathrm{MHz}, \mathrm{CDCl}_{3}\right): \delta=\delta 7.97-7.94(\mathrm{~m}, 1 \mathrm{H}), 7.94-7.89$ (comp, 3H), $7.64(\mathrm{dd}, J=8.5,1.8 \mathrm{~Hz}, 1 \mathrm{H}), 7.58-$ 7.50 (comp, 4H), 7.49-7.44 (comp, 2H), 7.38-7.33 (m, 1H), $4.36(\mathrm{dd}, J=6.7,4.2 \mathrm{~Hz}, 1 \mathrm{H}), 4.26$ (dd, $J=6.7,4.2 \mathrm{~Hz}$, 1H), 2.47 (br s, 1H), 2.24-2.00 (comp, 4H), 1.87-1.81 (comp, 2H).

${ }^{13}$ C-NMR (100 MHz, $\left.\mathrm{CDCl}_{3}\right): \delta=144.1,141.6,133.4,132.3,128.4,128.0,127.8,127.4,126.6,126.5,125.9,125.4$, $125.3,125.0,54.8,54.7,31.4,31.3,20.7$.

HRMS (ESI-TOF): Calculated for $\mathrm{C}_{21} \mathrm{H}_{22} \mathrm{~N}[\mathrm{M}+\mathrm{H}]^{+}: 288.1747$, found: 288.1733 . 
$\left(2 S^{*}, 6 S^{*}\right)-2-($ Naphthalen-1-yl)-6-phenylpiperidine

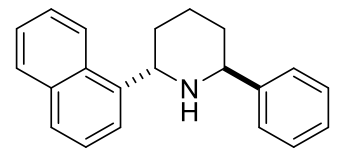

$( \pm)-1 r$

Following general procedure A, compound $( \pm)-\mathbf{1 r}$ was obtained from piperidine $(99 \mu \mathrm{L}, 1 \mathrm{mmol}$ ), trifluoroacetophenone $(147 \mu \mathrm{L}, 1.05 \mathrm{mmol})$, phenyl lithium $(1.07 \mathrm{mmol})$ (first substitution), trifluoroacetophenone (239 $\mu \mathrm{L}, 1.7 \mathrm{mmol}$ ), and 1-bromonaphthalene $(414.1 \mathrm{mg}, 2 \mathrm{mmol}$ ) (second substitution), as a yellow oil in $44 \%$ yield $(126.1 \mathrm{mg})$. Dichloromethane followed by hexanes containing ethyl acetate (10-30\%) was used as the eluent for silica gel chromatography.

\section{Characterization data for $( \pm)-1 \mathrm{r}$ :}

$\mathbf{R}_{\mathbf{f}}=0.46$ in hexanes/EtOAc $70: 30 \mathrm{v} / \mathrm{v}$.

${ }^{1}$ H-NMR $\left(500 \mathrm{MHz}, \mathrm{CDCl}_{3}\right): \delta=8.00-7.95(\mathrm{~m}, 1 \mathrm{H}), 7.88(\mathrm{dd}, J=7.5,1.4 \mathrm{~Hz}, 1 \mathrm{H}), 7.82(\mathrm{app} \mathrm{dt}, J=7.5,1.4 \mathrm{~Hz}$, $1 \mathrm{H}), 7.80-7.75(\mathrm{~m}, 1 \mathrm{H}), 7.53-7.38(\mathrm{comp}, 7 \mathrm{H}), 7.33-7.27(\mathrm{~m}, 1 \mathrm{H}), 4.77(\mathrm{dd}, J=8.7,3.6 \mathrm{~Hz}, 1 \mathrm{H}), 4.41$ (app t, $J=$ $4.5 \mathrm{~Hz}, 1 \mathrm{H}), 2.69$ (br s, 1H), 2.35-2.26 (m, 1H), 2.21-2.06 (comp, 2H), 2.00-1.88 (comp, 3H).

${ }^{13}$ C-NMR $\left(125 \mathrm{MHz}, \mathrm{CDCl}_{3}\right): \delta=143.3,140.6,133.9,130.9,128.9,128.5,127.4,127.0,126.5,125.8,125.5,125.3$, $123.3,123.0,55.2,50.6,32.7,29.3,21.4$.

HRMS (ESI-TOF): Calculated for $\mathrm{C}_{21} \mathrm{H}_{22} \mathrm{~N}[\mathrm{M}+\mathrm{H}]^{+}:$: 288.1747, found: 288.1673 . 
$\left(2 S^{*}, 6 S^{*}\right)$-2-(4-Fluorophenyl)-6-phenylpiperidine

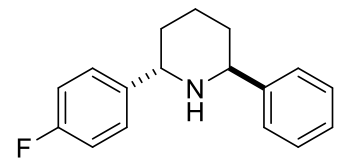

$( \pm)-1 s$

Following general procedure A, compound $( \pm)-\mathbf{1 s}$ was obtained from piperidine (99 $\mu \mathrm{L}, 1 \mathrm{mmol}$ ), trifluoroacetophenone $(147 \mu \mathrm{L}, 1.05 \mathrm{mmol})$, phenyl lithium $(1.07 \mathrm{mmol})$ (first substitution), trifluoroacetophenone ( $239 \mu \mathrm{L}, 1.7 \mathrm{mmol}$ ), and 1-bromo-4-fluorobenzene (220 $\mu \mathrm{L}, 2 \mathrm{mmol})$ (second substitution), as a yellow oil in $36 \%$ yield $(90.7 \mathrm{mg})$. Dichloromethane followed by hexanes containing ethyl acetate (10-30\%) was used as the eluent for silica gel chromatography.

\section{Characterization data for $( \pm)-1 \mathrm{~s}$ :}

$\mathbf{R}_{\mathbf{f}}=0.50$ in hexanes/EtOAc $70: 30 \mathrm{v} / \mathrm{v}$.

${ }^{1} \mathbf{H}-N M R\left(500 \mathrm{MHz}, \mathrm{CDCl}_{3}\right): \delta=7.49-7.37(\mathrm{comp}, 6 \mathrm{H}), 7.30$ (app t, $\left.J=7.0 \mathrm{~Hz}, 1 \mathrm{H}\right), 7.07$ (app tt, $J=8.6,1.9 \mathrm{~Hz}$, 2H), 4.16-4.11 (comp, 2H), 2.37 (br s, 1H), 2.11-1.89 (m, 4H), 1.77-1.72 (comp, 2H).

${ }^{13}$ C-NMR $\left(125 \mathrm{MHz}, \mathrm{CDCl}_{3}\right): \delta=161.6\left(\mathrm{~d}, J_{C-F}=245.0 \mathrm{~Hz}\right), 141.9\left(\mathrm{~d}, J_{C-F}=506.1 \mathrm{~Hz}\right), 128.5,128.3\left(\mathrm{~d}, J_{C-F}=7.8\right.$ $\mathrm{Hz}), 126.7,126.6(7), 126.6(0), 115.1\left(\mathrm{~d}, J_{C-F}=21.7 \mathrm{~Hz}\right), 54.7,54.2,31.7,31.1,20.6$.

${ }^{19}$ F-NMR $\left(470 \mathrm{MHz}, \mathrm{CDCl}_{3}\right): \delta=-116.2$.

HRMS (ESI-TOF): Calculated for $\mathrm{C}_{17} \mathrm{H}_{19} \mathrm{NF}[\mathrm{M}+\mathrm{H}]^{+}:$256.1496, found: 256.1484 . 
$\left(2 \mathrm{~S} * 6 \mathrm{~S}^{*}\right)$-2-(4-Bromophenyl)-6-phenylpiperidine

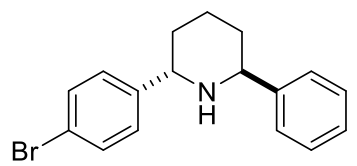

$( \pm)-1 t$

Following general procedure A, compound ( \pm )-1t was obtained from piperidine (99 $\mu \mathrm{L}, 1 \mathrm{mmol}$ ), trifluoroacetophenone $(147 \mu \mathrm{L}, 1.05 \mathrm{mmol})$, phenyl lithium $(1.07 \mathrm{mmol})$ (first substitution), trifluoroacetophenone (239 $\mu \mathrm{L}, 1.7 \mathrm{mmol}$ ), and 1,4-dibromobenzene ( $471.8 \mathrm{mg}, 2 \mathrm{mmol}$ ) (second substitution), as a colorless oil in $54 \%$ yield $(170.7 \mathrm{mg})$. Dichloromethane followed by hexanes containing ether $(6-18 \%)$ was used as the eluent for silica gel chromatography.

\section{Characterization data for $( \pm)-1 \mathrm{t}$ :}

$\mathbf{R}_{\mathbf{f}}=0.22$ in hexanes $/ \mathrm{Et}_{2} \mathrm{O} 80: 20 \mathrm{v} / \mathrm{v}$.

${ }^{1}$ H-NMR (600 MHz, CDCl $)$ ): $\delta=7.52-7.49$ (comp, 2H), 7.45-7.42 (comp, 2H), 7.41-7.37 (comp, 2H), 7.36-7.32 (comp, 2H), 7.31-7.27 (m, 1H), 4.13-4.09 (comp, 2H), 2.26-1.90 (comp, 5H), 1.77-1.67 (comp, 2H).

${ }^{13}$ C-NMR (151 MHz, $\left.\mathrm{CDCl}_{3}\right): \delta=143.9,143.2,131.5,128.6,128.5,126.7,126.6,120.3,54.7,54.3,31.4,31.3,20.6$.

HRMS (ESI-TOF): Calculated for $\mathrm{C}_{17} \mathrm{H}_{19} \mathrm{~N}^{79} \mathrm{Br}[\mathrm{M}+\mathrm{H}]^{+}: 316.0695$, found: 316.0666 . 
$\left(2 S^{*}, 6 S^{*}\right)$-2-(4-Methoxyphenyl)-6-(m-tolyl)piperidine

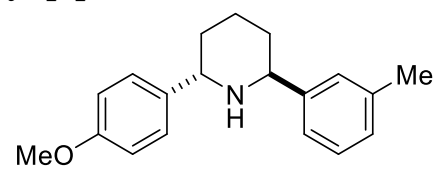

$( \pm)-1 u$

Following general procedure A, compound ( \pm )-1u was obtained from piperidine (99 $\mu \mathrm{L}, 1 \mathrm{mmol}$ ), trifluoroacetophenone (147 $\mu \mathrm{L}, 1.05 \mathrm{mmol})$, 3-bromotoluene $(130 \mu \mathrm{L}, 1.07 \mathrm{mmol})$ (first substitution), trifluoroacetophenone ( $239 \mu \mathrm{L}, 1.7 \mathrm{mmol})$, and 4-bromoanisole $(250 \mu \mathrm{L}, 2 \mathrm{mmol})$ (second substitution), as an orange oil in $43 \%$ yield $(120.5 \mathrm{mg})$. Dichloromethane followed by hexanes containing ethyl acetate (10-30\%) was used as the eluent for silica gel chromatography.

\section{Characterization data for $( \pm)-1 u$ :}

$\mathbf{R}_{\mathbf{f}}=0.32$ in hexanes/EtOAc 70:30 v/v.

${ }^{1}$ H-NMR $\left(500 \mathrm{MHz}, \mathrm{CDCl}_{3}\right): \delta=7.40-7.36$ (comp, 2H), 7.31-7.23 (comp, 3H), 7.12-7.08 (m, 1H), 6.97-6.92 (comp, 2H), 4.14-4.09 (comp, 2H), 3.85 (s, 3H), 2.41 (s, 3H), 2.34 (br s, 1H), 2.09-1.88 (comp, 4H), 1.78-1.71 (comp, 2H).

${ }^{13}$ C-NMR $\left(125 \mathrm{MHz}, \mathrm{CDCl}_{3}\right): \delta=158.2,144.1,138.0,136.1,128.3,127.8,127.4,127.3,123.7,113.8,55.2,54.7$, $54.2,31.5,31.2,21.5,20.7$.

HRMS (ESI-TOF): Calculated for $\mathrm{C}_{19} \mathrm{H}_{24} \mathrm{NO}[\mathrm{M}+\mathrm{H}]^{+}:$282.1852, found: 282.1837 . 
3-((2S*,6S*)-6-Phenylpiperidin-2-yl)pyridine

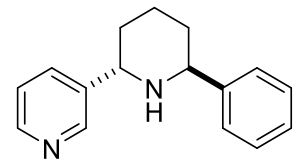

$( \pm)-1 v$

Following general procedure A, compound $( \pm)-\mathbf{1 v}$ was obtained from piperidine $(99 \mu \mathrm{L}, 1 \mathrm{mmol})$, trifluoroacetophenone $(147 \mu \mathrm{L}, 1.05 \mathrm{mmol})$, phenyl lithium $(1.07 \mathrm{mmol})$ (first substitution), trifluoroacetophenone (239 $\mu \mathrm{L}, 1.7 \mathrm{mmol})$, and 3-bromopyridine $(192.7 \mu \mathrm{L}, 2 \mathrm{mmol})$ (second substitution), as a yellow oil in $40 \%$ yield $(95.3 \mathrm{mg})$. Hexanes containing ethyl acetate $(50-100 \%)$ was used followed by ethyl acetate containing methanol $(1-4 \%)$ and isopropylamine (1\%) was used as the eluent for silica gel chromatography. Compound ( \pm )-1v is known, and the published characterization data matched our own in all respects. ${ }^{3}$ 


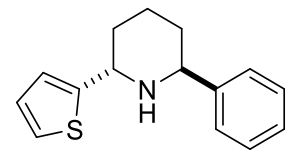

$$
( \pm)-1 w
$$

Following general procedure A, compound $( \pm)-\mathbf{1 w}$ was obtained from piperidine (99 $\mu \mathrm{L}, 1 \mathrm{mmol})$, trifluoroacetophenone $(147 \mu \mathrm{L}, 1.05 \mathrm{mmol})$, phenyl lithium $(1.07 \mathrm{mmol})$ (first substitution), trifluoroacetophenone $(239 \mu \mathrm{L}, 1.7 \mathrm{mmol})$, and thiophene ( $160 \mu \mathrm{L}, 2 \mathrm{mmol})$ (second substitution), as a colorless oil in $30 \%$ yield $(73 \mathrm{mg})$. Dichloromethane followed by hexanes containing ether (5-20\%) was used as the eluent for silica gel chromatography.

\section{Characterization data for $( \pm)-1 \mathrm{w}$ :}

$\mathbf{R}_{\mathbf{f}}=0.26$ in hexanes $/ \mathrm{Et}_{2} \mathrm{O} 70: 30 \mathrm{v} / \mathrm{v}$.

${ }^{1} \mathbf{H}$-NMR $\left(600 \mathrm{MHz}, \mathrm{CDCl}_{3}\right): \delta=7.48-7.45$ (comp, 2H), 7.41-7.37 (comp, 2H), 7.33-7.28 (comp, 2H), 7.05 (dd, $J$ $=5.1,3.5 \mathrm{~Hz}, 1 \mathrm{H}), 7.01-6.97(\mathrm{~m}, 1 \mathrm{H}), 4.59(\mathrm{app} \mathrm{t}, J=4.2 \mathrm{~Hz}, 1 \mathrm{H}), 4.12(\mathrm{dd}, J=9.2,3.1 \mathrm{~Hz}, 1 \mathrm{H}), 2.2(\mathrm{br} \mathrm{s}, 1 \mathrm{H})$, 2.17-2.07 (comp, 2H), 1.92-1.77 (comp, 4H).

${ }^{13}$ C-NMR $\left(150 \mathrm{MHz}, \mathrm{CDCl}_{3}\right): \delta=149.2,144.6,128.4,126.9,126.8,126.7,123.9,123.6,54.8,52.5,33.0,30.7,20.6$.

HRMS (ESI-TOF): Calculated for $\mathrm{C}_{15} \mathrm{H}_{18} \mathrm{NS}[\mathrm{M}+\mathrm{H}]^{+}:$244.1154, found: 244.1128 .

Note: Due to multiple spots on TLC with a similar $\mathrm{R}_{\mathrm{f}}$ as the desired compound, it is recommended to perform an acid-base wash for this substrate prior to column chromatography. 


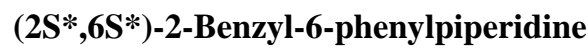

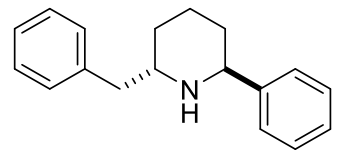

$( \pm)-1 x$

Following general procedure A, compound $( \pm)-\mathbf{1 x}$ was obtained from piperidine (99 $\mu \mathrm{L}, 1 \mathrm{mmol})$, trifluoroacetophenone $(147 \mu \mathrm{L}, 1.05 \mathrm{mmol})$, phenyl lithium (1.07 mmol) (first substitution), trifluoroacetophenone $(239 \mu \mathrm{L}, 1.7 \mathrm{mmol})$, and toluene $(213 \mu \mathrm{L}, 2 \mathrm{mmol})$ (second substitution), as a colorless solid in $40 \%$ yield (101 mg). Dichloromethane followed by hexanes containing ethyl acetate (10-30\%) was used as the eluent for silica gel chromatography.

\section{Characterization data for $( \pm)-1 x$ :}

$\mathbf{R}_{\mathbf{f}}=0.34$ in hexanes/EtOAc $70: 30 \mathrm{v} / \mathrm{v}$.

m.p.: $55-56^{\circ} \mathrm{C}$

${ }^{1} \mathbf{H}-\mathbf{N M R}\left(500 \mathrm{MHz}, \mathrm{CDCl}_{3}\right): \delta=7.35-7.31$ (comp, 6H), 7.27-7.21 (comp, 4H), 4.12 (app dt, $\left.J=8.7,2.5 \mathrm{~Hz}, 1 \mathrm{H}\right)$, $3.40-3.31(\mathrm{~m}, 1 \mathrm{H}), 3.07$ (dd, $J=13.5,9.2 \mathrm{~Hz}, 1 \mathrm{H}), 2.83$ (dd, $J=13.3,5.4 \mathrm{~Hz}, 1 \mathrm{H}), 2.05$ (br s, 1H), $1.95-1.76$ (comp, 4H), 1.75-1.58 (comp, 2H).

${ }^{13}$ C-NMR $\left(125 \mathrm{MHz}, \mathrm{CDCl}_{3}\right): \delta=144.7,139.7,129.0,128.5,128.3,126.8,126.7,126.1,54.3,53.9,38.8,33.1$, $29.4,20.2$.

HRMS (ESI-TOF): Calculated for $\mathrm{C}_{18} \mathrm{H}_{22} \mathrm{~N}[\mathrm{M}+\mathrm{H}]^{+}:$252.1747, found: 252.1718 . 


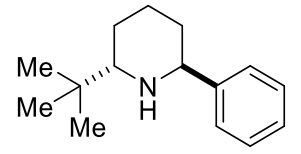

$( \pm)-1 y$

Following general procedure B, compound ( \pm )-1y was obtained from piperidine (99 $\mu \mathrm{L}, 1 \mathrm{mmol})$, trifluoroacetophenone $(147 \mu \mathrm{L}, 1.05 \mathrm{mmol})$, phenyl lithium $(1.07 \mathrm{mmol})$ (first substitution), trifluoroacetophenone $(239 \mu \mathrm{L}, 1.7 \mathrm{mmol})$, and $t$-butyl lithium ( $2 \mathrm{mmol})$ (second substitution), as a colorless oil in $40 \%$ yield (86.5 mg). Dichloromethane followed by hexanes containing ethyl acetate (10-30\%) was used as the eluent for silica gel chromatography.

\section{Characterization data for $( \pm)-1 \mathrm{y}$ :}

$\mathbf{R}_{\mathbf{f}}=0.31$ in hexanes/EtOAc 80:20 v/v.

${ }^{1} \mathbf{H}-\mathbf{N M R}\left(500 \mathrm{MHz}, \mathrm{CDCl}_{3}\right): \delta=7.51-7.41(\mathrm{~m}, 2 \mathrm{H}), 7.36($ app dd, $J=8.4,6.8 \mathrm{~Hz}, 2 \mathrm{H}), 7.22(\operatorname{app} \mathrm{dd}, J=8.4,6.8$ $\mathrm{Hz}, 1 \mathrm{H}), 4.39-4.22$ (m, 1H), 2.43-2.20 (comp, 2H), 2.04-1.77 (comp, 2H), 1.69 (app dq, J=13.8, $3.5 \mathrm{~Hz}, 1 \mathrm{H}), 1.63$ $(\mathrm{dd}, J=12.4,3.5 \mathrm{~Hz}, 1 \mathrm{H}), 1.49$ (app qt, $J=13.0,3.7 \mathrm{~Hz}, 1 \mathrm{H}), 1.21$ (app tdd, $J=12.5,11.3,3.9 \mathrm{~Hz}, 1 \mathrm{H}), 0.93$ (s, 9H).

${ }^{13}$ C-NMR $\left(125 \mathrm{MHz}, \mathrm{CDCl}_{3}\right): \delta=143.4,128.3,126.9,125.8,58.6,54.3,33.6,28.7,27.0,26.4,20.8$.

HRMS (ESI-TOF): Calculated for $\mathrm{C}_{15} \mathrm{H}_{24} \mathrm{~N}[\mathrm{M}+\mathrm{H}]^{+}:$218.1903, found: 218.1845 . 


\section{(2S*,7S*)-2-(4-Methoxyphenyl)-7-phenylazepane}

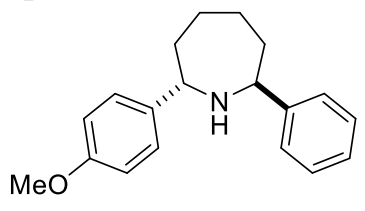

$( \pm)-1 z$

Following general procedure A, compound $( \pm)-\mathbf{1 z}$ was obtained from azepane $(113 \mu \mathrm{L}, 1 \mathrm{mmol})$, trifluoroacetophenone $(147 \mu \mathrm{L}, 1.05 \mathrm{mmol})$, phenyl lithium $(1.07 \mathrm{mmol})$ (first substitution), $t$-butyl phenyl ketone (201 $\mu \mathrm{L}, 1.2 \mathrm{mmol}$ ), and 4-bromoanisole (188 $\mu \mathrm{L}, 1.5 \mathrm{mmol})$ (second substitution), as a colorless oil in $34 \%$ yield $(95.7 \mathrm{mg})$. Dichloromethane followed by hexanes containing ether $(15-30 \%)$ was used as the eluent for silica gel chromatography. Compound $( \pm)-\mathbf{1 z}$ is known, and the published characterization data matched our own in all respects. $^{3}$ 


\section{$(2 R *, 7 R *)-2,7-D i b u t y l a z e p a n e$}

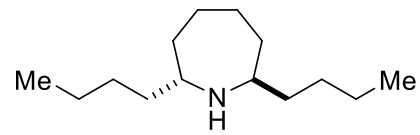

$( \pm)-1$ aa

Following general procedure B, compound ( \pm )-1aa was obtained from azepane (113 $\mu \mathrm{L}, 1 \mathrm{mmol})$, trifluoroacetophenone $(147 \mu \mathrm{L}, 1.05 \mathrm{mmol}), n$-butyl lithium $(1.2 \mathrm{mmol})$ (first substitution), $t$-butyl phenyl ketone $(201 \mu \mathrm{L}, 1.2 \mathrm{mmol})$, and $n$-butyl lithium (1.5 mmol) (second substitution), as a colorless oil in $49 \%$ yield (103.7 mg). Hexanes containing ethyl acetate (30-60\%) was used as the eluent for silica gel chromatography.

\section{Characterization data for $( \pm)$-1aa:}

$\mathbf{R}_{\mathbf{f}}=0.18$ in hexanes/EtOAc 50:50 v/v.

${ }^{1} \mathbf{H}-\mathrm{NMR}\left(500 \mathrm{MHz}, \mathrm{CDCl}_{3}\right): \delta=2.63$ (app ddt, $\left.J=13.0,6.5,3.2 \mathrm{~Hz}, 2 \mathrm{H}\right), 1.82-1.67$ (comp, 4H), $1.54-1.04$ (comp, $17 \mathrm{H}), 0.86(\mathrm{t}, J=7.0 \mathrm{~Hz}, 6 \mathrm{H})$.

${ }^{13}$ C-NMR $\left(125 \mathrm{MHz}, \mathrm{CDCl}_{3}\right): \delta=53.7,37.7,36.9,29.0,27.8,22.8,14.0$.

HRMS (ESI-TOF): Calculated for $\mathrm{C}_{14} \mathrm{H}_{30} \mathrm{~N}[\mathrm{M}+\mathrm{H}]^{+}:$212.2373, found: 212.2354. 
(2S*,5S*)-2-(4-Methoxyphenyl)-5-(4-(trifluoromethyl)phenyl)pyrrolidine

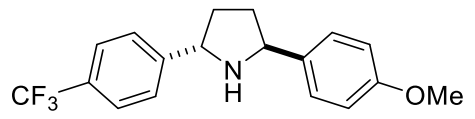

$( \pm)-1 a b$

Following general procedure A, compound ( \pm )-1ab was obtained from pyrrolidine ( $82 \mu \mathrm{L}, 1 \mathrm{mmol}$ ), benzophenone (191 mg, $1.05 \mathrm{mmol}$ ), 4-bromoanisole (200 mg, $1.07 \mathrm{mmol}$ ) (first substitution), $t$-butyl phenyl ketone (201 $\mu \mathrm{L}, 1.2$ mmol), and 4-bromobenzotrifluoride ( $210 \mu \mathrm{L}, 1.5 \mathrm{mmol}$ ) (second substitution), as a colorless oil in 32\% yield (103 $\mathrm{mg}$ ). Dichloromethane followed by hexanes containing ethyl acetate $(5-25 \%)$ was used as the eluent for silica gel chromatography.

\section{Characterization data $( \pm)-1 a b:$}

$\mathbf{R}_{\mathbf{f}}=0.36$ in hexanes/EtOAc 80:20 v/v.

${ }^{1}$ H-NMR $\left(500 \mathrm{MHz}, \mathrm{CDCl}_{3}\right): \delta=7.59(\mathrm{~d}, J=8.3 \mathrm{~Hz}, 2 \mathrm{H}), 7.53(\mathrm{~d}, J=8.3,2 \mathrm{H}), 7.35-7.31(\mathrm{~m}, 2 \mathrm{H}), 6.94-6.85(\mathrm{~m}$, $2 \mathrm{H}), 4.61(\operatorname{app~t}, J=7.3 \mathrm{~Hz}, 1 \mathrm{H}), 4.48(\mathrm{dd}, J=7.9,6.5 \mathrm{~Hz}, 1 \mathrm{H}), 3.81(\mathrm{~s}, 3 \mathrm{H}), 2.48-2.41(\mathrm{~m}, 1 \mathrm{H}), 2.41-2.32(\mathrm{~m}, 1 \mathrm{H})$, $2.18(\mathrm{~s}, 1 \mathrm{H}), 1.97-1.81(\mathrm{comp}, 2 \mathrm{H})$.

${ }^{13}$ C-NMR $\left(125 \mathrm{MHz}, \mathrm{CDCl}_{3}\right): \delta=158.8,150.2,137.4,129.1\left(\mathrm{q}, J_{C-F}=32.1 \mathrm{~Hz}\right), 127.6,126.8,125.48\left(\mathrm{q}, J_{C-F}=3.7\right.$ $\mathrm{Hz}), 114.1,62.1,61.8,55.4,35.8,35.5$.

${ }^{19}$ F-NMR $\left(470 \mathrm{MHz}, \mathrm{CDCl}_{3}\right): \delta=-62.3$

HRMS (ESI-TOF): Calculated for $\mathrm{C}_{18} \mathrm{H}_{19} \mathrm{~F}_{3} \mathrm{NO}[\mathrm{M}+\mathrm{H}]^{+}$: 322.1413, found: 322.1386 . 
$\left(2 S^{*}, 5 S^{*}\right)$-2-(4-Bromophenyl)-5-(m-tolyl)pyrrolidine

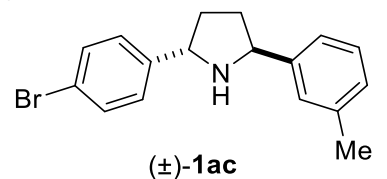

Following general procedure A, compound ( \pm )-1ac was obtained from pyrrolidine ( $82 \mu \mathrm{L}, 1 \mathrm{mmol}$ ), benzophenone (191.3 mg, $1.05 \mathrm{mmol})$, 3-bromotoluene (130 $\mu \mathrm{L}, 1.07 \mathrm{mmol})$ (first substitution), $t$-butyl phenyl ketone (201 $\mu \mathrm{L}, 1.2$ $\mathrm{mmol}$ ), and 1,4-dibromobenzene (353.85 $\mathrm{mg}, 1.5 \mathrm{mmol}$ ) (second substitution), as a yellow oil in 32\% yield (101 $\mathrm{mg}$ ). Dichloromethane followed by hexanes containing ethyl acetate (10-30\%) was used as the eluent for silica gel chromatography.

\section{Characterization data ( \pm )-1ac:}

$\mathbf{R}_{\mathbf{f}}=0.44$ in hexanes/EtOAc $85: 15 \mathrm{v} / \mathrm{v}$.

${ }^{1} \mathbf{H}-\mathrm{NMR}\left(600 \mathrm{MHz}, \mathrm{CDCl}_{3}\right.$ ): $\delta=7.54-7.51$ (comp, 2H), 7.39-7.36 (comp, 2H), 7.34-7.29 (comp, 2H), 7.28-7.25 (m, 1H), $7.16(\mathrm{~d}, J=7.4 \mathrm{~Hz}, 1 \mathrm{H}), 4.58($ app t, $J=7.3 \mathrm{~Hz}, 1 \mathrm{H}), 4.55$ (app t, $J=7.2 \mathrm{~Hz}, 1 \mathrm{H}), 2.49-2.41$ (comp, 5H), 2.26 (br s, 1H), 2.02-1.85 (comp, 2H).

${ }^{13}$ C-NMR $\left(151 \mathrm{MHz}, \mathrm{CDCl}_{3}\right): \delta=145.4,144.9,138.0,131.3,128.3,128.0,127.5,126.9,123.2,120.2,62.2,61.4$, 35.6, 35.3, 21.4.

HRMS (ESI-TOF): Calculated for $\mathrm{C}_{17} \mathrm{H}_{19} \mathrm{~N}^{79} \mathrm{Br}[\mathrm{M}+\mathrm{H}]^{+}: 316.0695$, found: 316.0677 . 
$\left(2 S^{*}, 8 S^{*}\right)$-2-(Naphthalen-2-yl)-8-phenylazocane

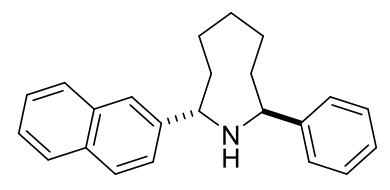

( \pm -1 ad

Following general procedure A, compound ( \pm )-1ad was obtained from azocane (126 $\mu \mathrm{L}, 1 \mathrm{mmol})$, benzophenone (191.3 mg, $1.05 \mathrm{mmol})$, phenyl lithium (1.07 mmol) (first substitution), $t$-butyl phenyl ketone (201 $\mu \mathrm{L}, 1.2 \mathrm{mmol})$, and 2-bromonaphthalene (310.6 mg, $1.5 \mathrm{mmol}$ ) (second substitution), as a colorless oil in 50\% yield (157.7 $\mathrm{mg}$ ). Dichloromethane followed by hexanes containing ether (2-8\%) was used as the eluent for silica gel chromatography.

\section{Characterization data $( \pm)-1$ ad:}

$\mathbf{R}_{\mathbf{f}}=0.57$ in hexanes $/ \mathrm{Et}_{2} \mathrm{O} 80: 20 \mathrm{v} / \mathrm{v}$.

${ }^{1} \mathbf{H}-\mathbf{N M R}\left(400 \mathrm{MHz}, \mathrm{CDCl}_{3}\right): \delta=7.82-7.77(\mathrm{~m}, 1 \mathrm{H}), 7.76-7.68(\mathrm{comp}, 3 \mathrm{H}), 7.49-7.41(\mathrm{comp}, 2 \mathrm{H}), 7.38(\mathrm{dd}, J=$ $8.5,1.8 \mathrm{~Hz}, 1 \mathrm{H}), 7.31-7.23$ (comp, 4H), 7.22-7.16 (m, 1H), 4.26 (dd, $J=10.0,4.0 \mathrm{~Hz}, 1 \mathrm{H}), 4.14$ (dd, $J=10.2,3.5$ $\mathrm{Hz}, 1 \mathrm{H}), 2.16-1.86$ (comp, 9H), 1.84-1.74 (comp, 2H).

${ }^{13}$ C-NMR $\left(100 \mathrm{MHz}, \mathrm{CDCl}_{3}\right): \delta=146.7,144.0,133.3,132.3,128.3,127.8,127.4,126.7(2), 126.6(9), 126.4$, 125.8(2), 125.7(8), 125.3, 124.7, 58.1(5), 58.1(4), 34.4, 34.3, 27.8, 25.8(3), 25.7(9).

HRMS (ESI-TOF): Calculated for $\mathrm{C}_{23} \mathrm{H}_{26} \mathrm{~N}[\mathrm{M}+\mathrm{H}]^{+}: 316.2060$, found: 316.2054 . 


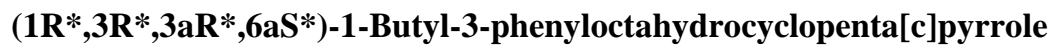

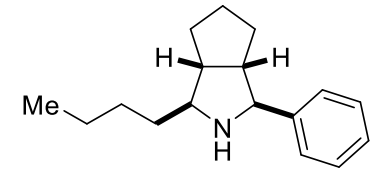

$( \pm)-1$ ae

Following general procedure B, compound $( \pm)$-1ae was obtained from octahydrocyclopenta[c]pyrrole (111 mg, 1 $\mathrm{mmol})$, trifluoroacetophenone $(147 \mu \mathrm{L}, 1.05 \mathrm{mmol})$, phenyl lithium $(1.07 \mathrm{mmol})$ (first substitution), trifluoroacetophenone ( $239 \mu \mathrm{L}, 1.7 \mathrm{mmol})$, and $n$-butyl lithium $(2 \mathrm{mmol})$ (second substitution), as a colorless oil in $45 \%$ yield $(109.5 \mathrm{mg})$. Dichloromethane followed by hexanes containing ether (6-18\%) was used as the eluent for silica gel chromatography.

\section{Characterization data $( \pm)$-1ae:}

$\mathbf{R}_{\mathbf{f}}=0.40$ in hexanes $/ \mathrm{Et}_{2} \mathrm{O} 70: 30 \mathrm{v} / \mathrm{v}$.

${ }^{1}$ H-NMR (400 MHz, $\mathrm{CDCl}_{3}$ ): $\delta=7.47-7.42$ (comp, 2H), 7.38-7.31 (comp, 2H), 7.29-7.24 (m, 1H), 3.59 (d, $J=8.4$ $\mathrm{Hz}, 1 \mathrm{H}), 2.68$ (ddd, $J=7.8,7.0,6.0 \mathrm{~Hz}, 1 \mathrm{H}), 2.52(\operatorname{app} \mathrm{dtd}, J=9.8,8.1,1.5 \mathrm{~Hz}, 1 \mathrm{H}), 2.32-2.30(\mathrm{~m}, 1 \mathrm{H}), 2.06$ (br s, 1H), 1.72-1.34 (comp, 12H), 0.98-0.92 (m, 3H).

${ }^{13}$ C-NMR $\left(100 \mathrm{MHz}, \mathrm{CDCl}_{3}\right): \delta=143.9,128.3,127.0,126.9,69.4,66.2,53.1,50.9,35.7,31.3,30.5,29.7,25.1$, 23.0, 14.1 .

HRMS (ESI-TOF): Calculated for $\mathrm{C}_{17} \mathrm{H}_{26} \mathrm{~N}[\mathrm{M}+\mathrm{H}]^{+}:$244.2060, found: 244.2037. 


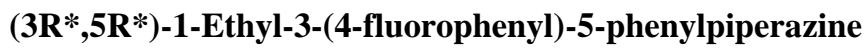

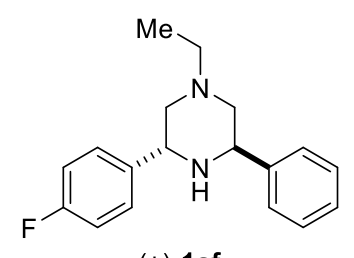

$( \pm)-1$ af

Following general procedure A, compound $( \pm)$-1af was obtained from 1-ethylpiperazine (127 $\mu \mathrm{L}, 1 \mathrm{mmol}$ ), trifluoroacetophenone $(147 \mu \mathrm{L}, 1.05 \mathrm{mmol})$, phenyl lithium $(1.07 \mathrm{mmol})$ (first substitution), trifluoroacetophenone ( $239 \mu \mathrm{L}, 1.7 \mathrm{mmol}$ ), and 1-bromo-4-fluorobenzene ( $350 \mathrm{mg}, 2 \mathrm{mmol}$ ) (second substitution), as a yellow oil in $42 \%$ yield $(119.4 \mathrm{mg})$. Dichloromethane followed by hexanes containing ether (5-20\%) was used as the eluent for silica gel chromatography.

\section{Characterization data for $( \pm)-1$ af:}

$\mathbf{R}_{\mathbf{f}}=0.31$ in hexanes $/ \mathrm{Et}_{2} \mathrm{O} 70: 30 \mathrm{v} / \mathrm{v}$.

${ }^{1} \mathbf{H}-\mathrm{NMR}\left(400 \mathrm{MHz}, \mathrm{CDCl}_{3}\right): \delta=7.58-7.51$ (comp, 4H), 7.39-7.34 (comp, 2H), 7.31-7.26 (m, 1H), 7.07-7.00 (comp, 2H), 4.19-4.10 (comp, 2H), 2.87-2.72 (comp, 4H), 2.53 (dq, $J=12.1,7.3 \mathrm{~Hz}, 1 \mathrm{H}$ ), 2.43 (dq, $J=12.2,7.1$ $\mathrm{Hz}, 1 \mathrm{H}), 2.05$ (br s, 1H), 1.18 (t, $J=7.2 \mathrm{~Hz}, 3 \mathrm{H})$.

${ }^{13}$ C-NMR $\left(100 \mathrm{MHz}, \mathrm{CDCl}_{3}\right): \delta=161.8\left(\mathrm{~d}, J_{C-F}=245.1 \mathrm{~Hz}\right), 143.0,138.9\left(\mathrm{~d}, J_{C-F}=2.7 \mathrm{~Hz}\right), 129.2,129.1,127.9(\mathrm{~d}$, $\left.J_{C-F}=89.5 \mathrm{~Hz}\right), 127.1,115.0\left(\mathrm{~d}, J_{C-F}=21.0 \mathrm{~Hz}\right), 58.1,57.9,54.5,54.0,52.6,12.0$.

${ }^{19}$ F-NMR $\left(377 \mathrm{MHz}, \mathrm{CDCl}_{3}\right): \delta=-116.0$

HRMS (ESI-TOF): Calculated for $\mathrm{C}_{18} \mathrm{H}_{22} \mathrm{~N}_{2} \mathrm{~F}[\mathrm{M}+\mathrm{H}]^{+}$: 285.1762, found: 285.1775. 


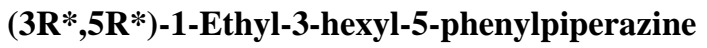

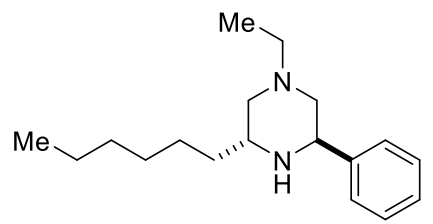

$( \pm)-1$ ag

Following general procedure B, compound ( \pm )-1ag was obtained from 1-ethylpiperazine (127 $\mu \mathrm{L}, 1 \mathrm{mmol}$ ), trifluoroacetophenone $(147 \mu \mathrm{L}, 1.05 \mathrm{mmol})$, phenyl lithium $(1.07 \mathrm{mmol})$ (first substitution), trifluoroacetophenone $(239 \mu \mathrm{L}, 1.7 \mathrm{mmol})$, and $n$-hexyl lithium ( $2 \mathrm{mmol}$ ) (second substitution), as a yellow oil in $43 \%$ yield (118 $\mathrm{mg}$ ). Dichloromethane followed by hexanes containing ether (10-25\%) was used as the eluent for silica gel chromatography.

\section{Characterization data for $( \pm)-1$ ag:}

$\mathbf{R}_{\mathbf{f}}=0.24$ in hexanes $/ \mathrm{Et}_{2} \mathrm{O} 70: 30 \mathrm{v} / \mathrm{v}$.

${ }^{1}$ H-NMR (400 MHz, CDCl $)$ ): $\delta=7.52-7.47$ (comp, 2H), 7.37-7.32 (comp, 2H), 7.30-7.25 (m, 1H), 4.17 (dd, $J=$ 8.2, 3.2 Hz, 1H), 3.03 (app ddt, $J=7.7,6.4,3.8 \mathrm{~Hz}, 1 \mathrm{H}$ ), 2.77 (dd, $J=11.0,2.7 \mathrm{~Hz}, 1 \mathrm{H}), 2.61-2.53(\mathrm{~m}, 1 \mathrm{H}), 2.52-$ 2.30 (comp, 4H), 1.93 (br s, 1H), 1.81-1.70 (m, 1H), 1.69-1.58 (m, 1H), 1.37-1.25 (comp, 8H), $1.11(\mathrm{t}, J=7.2 \mathrm{~Hz}$, $3 \mathrm{H}), 0.93-0.88(\mathrm{~m}, 3 \mathrm{H})$.

${ }^{13} \mathrm{C}-\mathrm{NMR}\left(100 \mathrm{MHz}, \mathrm{CDCl}_{3}\right): \delta=143.1,128.3,127.2,127.1,60.1,56.9,54.2,52.7,51.9,32.2,31.8,29.3,26.5$, 22.6, 14.0, 11.9.

HRMS (ESI-TOF): Calculated for $\mathrm{C}_{18} \mathrm{H}_{31} \mathrm{~N}_{2}[\mathrm{M}+\mathrm{H}]^{+}$: 275.2482, found: 275.2476. 
$\left(2 \mathrm{R}^{*}, 5 \mathrm{~S}^{*}\right)$-2-Butyl-5-phenylpyrrolidine and $\left(2 \mathrm{~S}^{*}, 5 \mathrm{~S}^{*}\right)$-2-Butyl-5-phenylpyrrolidine

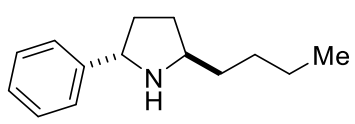

$( \pm)-1 \mathrm{ah}$

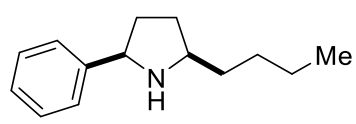

$( \pm)-1 a^{\prime}$

Following general procedure B, compound ( \pm )-1ah was obtained from pyrrolidine ( $82 \mu \mathrm{L}, 1 \mathrm{mmol})$, benzophenone (191.3 mg, $1.05 \mathrm{mmol}), n$-butyl lithium (1.07 mmol) (first substitution), $t$-butyl phenyl ketone (201 $\mu \mathrm{L}, 1.2 \mathrm{mmol})$, and phenyl lithium (1.5 mmol) (second substitution), in $40 \%$ combined yield $(81.3 \mathrm{mg}$ ) and 3:1 diastereomeric ratio. Dichloromethane followed by hexanes containing ethyl acetate (10-50\%) was used as the eluent for silica gel chromatography.

Characterization data for major diastereomer ( \pm )-1ah: a colorless oil in $30 \%$ yield $(61 \mathrm{mg})$

$\mathbf{R}_{\mathbf{f}}=0.21$ in hexanes/EtOAc 70:30 v/v.

${ }^{1} \mathbf{H}-\mathrm{NMR}\left(400 \mathrm{MHz}, \mathrm{CDCl}_{3}\right.$ ): $\delta=7.36-7.29$ (comp, 4H), 7.22 (app tt, $\left.J=7.0,1.6 \mathrm{~Hz}, 1 \mathrm{H}\right), 4.30$ (dd, $J=8.2,6.8 \mathrm{~Hz}$, 1H), 3.39-3.32 (m, 1H), 2.25 (dddd, $J=12.4,7.7,6.7,3.0 \mathrm{~Hz}, 1 \mathrm{H}$ ), 2.09 (dddd, $J=12.2,7.7,6.4,3.0 \mathrm{~Hz}, 1 \mathrm{H}$ ), 2.00 (br s, 1H), 1.76 (app ddt, $J=12.4,10.0,8.0 \mathrm{~Hz}, 1 \mathrm{H}), 1.58-1.26$ (comp, 7H), 0.93-0.90 (comp, 3H).

${ }^{13}$ C-NMR (100 MHz, $\left.\mathrm{CDCl}_{3}\right): \delta=145.9,128.3,126.6,126.3,61.4,58.9,37.0,35.2,32.9,29.5,22.8,14.1$.

HRMS (ESI-TOF): Calculated for $\mathrm{C}_{14} \mathrm{H}_{22} \mathrm{~N}[\mathrm{M}+\mathrm{H}]^{+}: 204.1747$, found: 204.1740 . 
Characterization data for minor diastereomer ( \pm )-1ah': a yellow oil in 10\% yield (20.3 mg)

$\mathbf{R}_{\mathbf{f}}=0.30$ in hexanes/EtOAc 70:30 v/v.

${ }^{1}$ H-NMR (400 MHz, $\mathrm{CDCl}_{3}$ ): $\delta=7.41-7.37$ (comp, 2H), 7.35-7.29 (comp, 2H), 7.25-7.20 (m, $\left.1 \mathrm{H}\right), 4.14$ (app t, $J=$ $7.9 \mathrm{~Hz}, 1 \mathrm{H}), 3.20-3.12(\mathrm{~m}, 1 \mathrm{H}), 2.16$ (dddd, $J=12.4,8.7,7.4,5.8 \mathrm{~Hz}, 1 \mathrm{H}), 2.06$ (br s, $1 \mathrm{H}), 1.98$ (dddd, $J=12.3,8.7$, 7.2, $6.0 \mathrm{~Hz}, 1 \mathrm{H}), 1.71$ (dddd, $J=12.4,10.2,8.4,6.0 \mathrm{~Hz}, 1 \mathrm{H}), 1.65-1.31$ (comp, 7H), 0.96-0.90 (m, 3H).

${ }^{13} \mathrm{C}-\mathrm{NMR}\left(100 \mathrm{MHz}, \mathrm{CDCl}_{3}\right): \delta=144.7,128.2,126.7,126.6,62.6,59.5,36.5,33.8,31.6,29.6,22.9,14.1$.

HRMS (ESI-TOF): Calculated for $\mathrm{C}_{14} \mathrm{H}_{22} \mathrm{~N}[\mathrm{M}+\mathrm{H}]^{+}:$204.1747, found: 204.1755. 


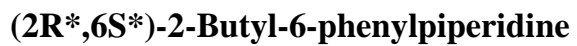

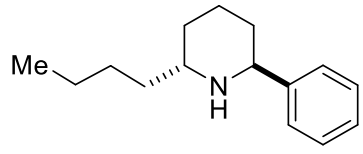

$( \pm)-1 \mathbf{a i}$

Following general procedure B, compound ( \pm )-1ai was obtained from piperidine $(99 \mu \mathrm{L}, 1 \mathrm{mmol}$ ), trifluoroacetophenone (147 $\mu \mathrm{L}, 1.05 \mathrm{mmol})$, phenyl lithium $(1.07 \mathrm{mmol})$ (first substitution), trifluoroacetophenone $(239 \mu \mathrm{L}, 1.7 \mathrm{mmol}$ ), and $n$-butyl lithium ( $2 \mathrm{mmol}$ ) (second substitution), as a yellow oil in 52\% yield (113.1 $\mathrm{mg}$ ). Dichloromethane followed by hexanes containing ethyl acetate (10-30\%) was used as the eluent for silica gel chromatography. Compound ( \pm )-1ai is known, and the published characterization data matched our own in all respects. $^{3}$ 


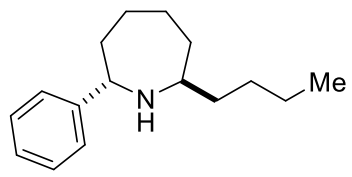

$( \pm)-1 \mathbf{a j}$

Following general procedure A, compound ( \pm )-1aj was obtained from azepane (113 $\mu \mathrm{L}, 1 \mathrm{mmol})$, trifluoroacetophenone $(147 \mu \mathrm{L}, 1.05 \mathrm{mmol}), n$-butyl lithium (1.07 mmol) (first substitution), $t$-butyl phenyl ketone $(201 \mu \mathrm{L}, 1.2 \mathrm{mmol})$, and phenyl lithium (1.5 mmol) (second substitution), as a yellow oil in $55 \%$ yield (126.8 mg). Dichloromethane followed by hexanes containing ethyl acetate (10-30\%) was used as the eluent for silica gel chromatography.

\section{Characterization data for ( \pm )-1aj:}

$\mathbf{R}_{\mathbf{f}}=0.30$ in hexanes/EtOAc $70: 30 \mathrm{v} / \mathrm{v}$.

${ }^{1} \mathbf{H}-\mathbf{N M R}\left(500 \mathrm{MHz}, \mathrm{CDCl}_{3}\right): \delta=7.37-7.29(\mathrm{comp}, 4 \mathrm{H}), 7.21(\mathrm{~m}, 1 \mathrm{H}), 3.94(\mathrm{dd}, J=10.9,2.9 \mathrm{~Hz}, 1 \mathrm{H}), 2.98(\mathrm{~m}, 1 \mathrm{H})$, 2.04-1.82 (comp, 5H), 1.81-1.72 (m, 1H), 1.55-1.32 (comp, 5H), 1.33-1.19 (comp, 4H), 0.87 (t, J=7.0 Hz, 3H).

${ }^{13} \mathrm{C}-\mathrm{NMR}\left(125 \mathrm{MHz}, \mathrm{CDCl}_{3}\right): \delta=147.6,128.4,126.4,126.3,58.2,54.9,38.0,37.3,36.4,28.8,28.7,27.3,22.8$, 14.0 .

HRMS (ESI-TOF): Calculated for $\mathrm{C}_{16} \mathrm{H}_{26} \mathrm{~N}[\mathrm{M}+\mathrm{H}]^{+}:$232.2060, found: 232.2052. 
2D-NMR Analysis for major diastereomer ( \pm )-1d, Selected Interactions (in $\mathrm{CDCl}_{3}$ )

cosY

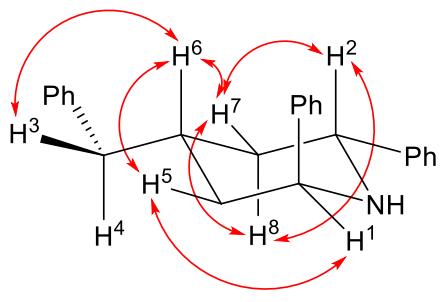

\begin{tabular}{|l|l|}
\hline Protons & Chemical Shifts (ppm) \\
\hline $\mathrm{H}^{1}$ & $4.52-4.49$ \\
\hline $\mathrm{H}^{2}$ & 3.84 \\
\hline $\mathrm{H}^{3}$ & 2.67 \\
\hline $\mathrm{H}^{4}$ & 2.61 \\
\hline $\mathrm{H}^{5}$ & $2.47-2.40$ \\
\hline $\mathrm{H}^{6}$ & 2.06 \\
\hline $\mathrm{H}^{7}$ & $1.88-1.78$ \\
\hline $\mathrm{H}^{8}$ & 1.39 \\
\hline
\end{tabular}

2D-NMR Analysis for minor diastereomer ( \pm )-1d', Selected Interactions (in $\mathrm{CDCl}_{3}$ )

$\cos Y$

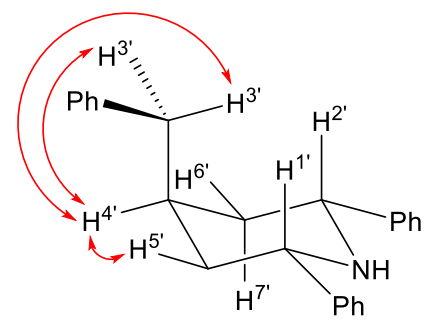

NOESY

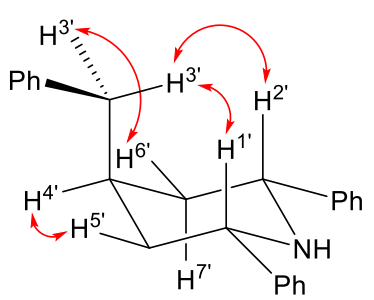

\begin{tabular}{|l|l|}
\hline Protons & Chemical Shifts (ppm) \\
\hline $\mathrm{H}^{1^{\prime}, 2^{\prime}}$ & 4.48 \\
\hline $\mathrm{H}^{3^{\prime}}$ & 3.27 \\
\hline $\mathrm{H}^{4^{\prime}}$ & $2.66-2.57$ \\
\hline $\mathrm{H}^{4^{\prime}}$ & 1.98 \\
\hline $\mathrm{H}^{5^{\prime}, 6^{\prime}, 7^{\prime}}$ & $2.09-1.93$ \\
\hline
\end{tabular}


2D-NMR Analysis of ( \pm )-1e, Selected Interactions (in $\mathrm{CDCl}_{3}$ )

GcosY

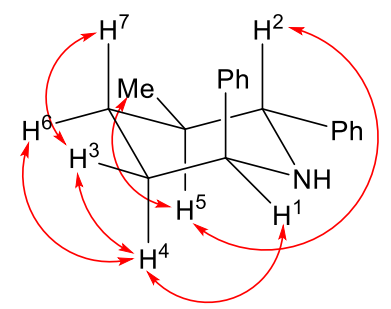

NOESY

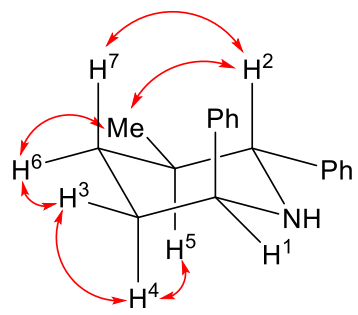

\begin{tabular}{|l|l|}
\hline Protons & Chemical Shifts (ppm) \\
\hline $\mathrm{H}^{1}$ & 4.38 \\
\hline $\mathrm{H}^{2}$ & 3.47 \\
\hline $\mathrm{H}^{3}$ & 2.41 \\
\hline $\mathrm{H}^{4}$ & $2.32-2.18$ \\
\hline $\mathrm{H}^{5}$ & $1.96-1.84$ \\
\hline $\mathrm{H}^{6}$ & 1.79 \\
\hline $\mathrm{H}^{7}$ & 1.37 \\
\hline $\mathrm{Me}$ & 0.69 \\
\hline
\end{tabular}


2D-NMR Analysis of ( \pm )-1x, Selected Interactions (in $\mathrm{CDCl}_{3}$ )
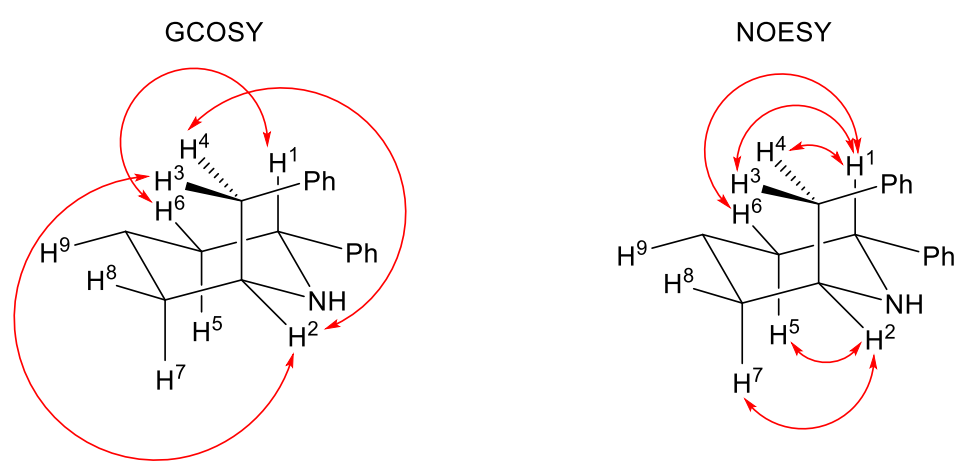

\begin{tabular}{|l|l|}
\hline Protons & Chemical Shifts (ppm) \\
\hline $\mathrm{H}^{1}$ & 4.13 \\
\hline $\mathrm{H}^{2}$ & 3.36 \\
\hline $\mathrm{H}^{3}$ & 3.08 \\
\hline $\mathrm{H}^{4}$ & 2.84 \\
\hline $\mathrm{H}^{5,6,7,8}$ & $1.80-1.60$ \\
\hline
\end{tabular}




\section{D-NMR Analysis of ( \pm )-1ag, Selected Interactions (in $\mathrm{CDCl}_{3}$ )}

GcosY

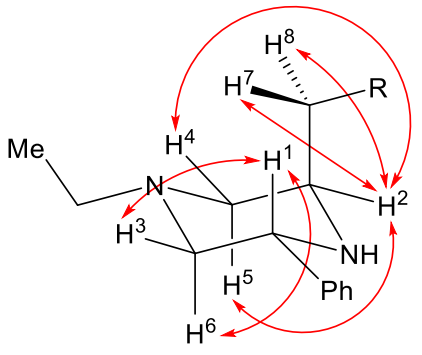

$\mathrm{R}=n$-pentyl

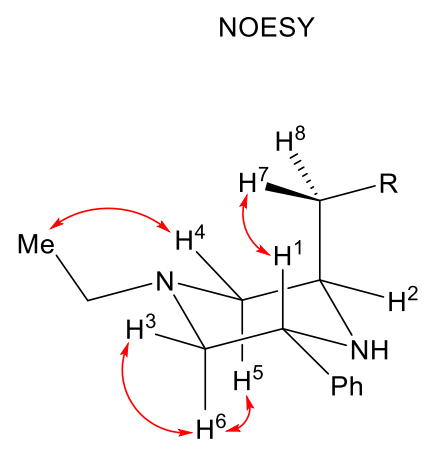

$\mathrm{R}=n$-pentyl

\begin{tabular}{|l|l|}
\hline Protons & Chemical Shifts (ppm) \\
\hline $\mathrm{H}^{1}$ & 4.17 \\
\hline $\mathrm{H}^{2}$ & 3.03 \\
\hline $\mathrm{H}^{3}$ & 2.77 \\
\hline $\mathrm{H}^{4}$ & $2.61-2.53$ \\
\hline $\mathrm{H}^{5}$ & $2.52-2.4$ \\
\hline $\mathrm{H}^{6}$ & $2.4-2.3$ \\
\hline $\mathrm{H}^{7}$ & $1.81-1.70$ \\
\hline $\mathrm{H}^{8}$ & $1.69-1.58$ \\
\hline
\end{tabular}


2D-NMR Analysis for major diastereomer ( \pm )-1ah, Selected Interactions (in $\mathrm{CDCl}_{3}$ )
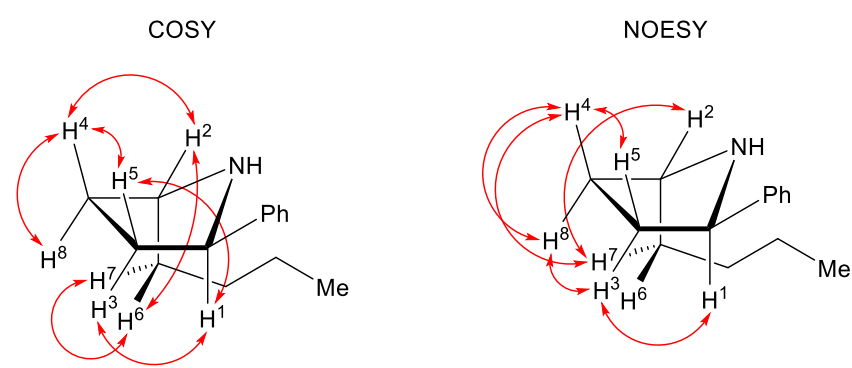

\begin{tabular}{|l|l|}
\hline Protons & Chemical Shifts (ppm) \\
\hline $\mathrm{H}^{1}$ & 4.33 \\
\hline $\mathrm{H}^{2}$ & 3.38 \\
\hline $\mathrm{H}^{3}$ & 2.28 \\
\hline $\mathrm{H}^{4}$ & 2.11 \\
\hline $\mathrm{H}^{5}$ & 1.78 \\
\hline $\mathrm{H}^{6}$ & $1.61-1.53$ \\
\hline $\mathrm{H}^{7,8}$ & $1.52-1.43$ \\
\hline
\end{tabular}

2D-NMR Analysis for major diastereomer ( \pm )-1ah, Selected Interactions (in $\mathrm{CDCl}_{3}$ )
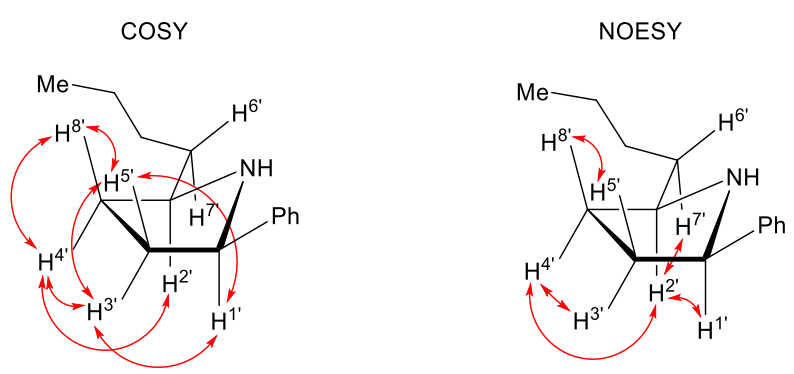

\begin{tabular}{|l|l|}
\hline Protons & Chemical Shifts (ppm) \\
\hline $\mathrm{H}^{1^{\prime}}$ & 4.14 \\
\hline $\mathrm{H}^{2}$ & $3.2-3.12$ \\
\hline $\mathrm{H}^{3^{\prime}}$ & 2.16 \\
\hline $\mathrm{H}^{4^{\prime}}$ & 1.98 \\
\hline $\mathrm{H}^{5^{\prime}}$ & 1.71 \\
\hline $\mathrm{H}^{6^{\prime}}$ & $1.65-1.55$ \\
\hline $\mathrm{H}^{7^{\prime} 8^{\prime}}$ & $1.54-1.45$ \\
\hline
\end{tabular}




\section{Crystallographic Summary for Compound ( \pm )-11}

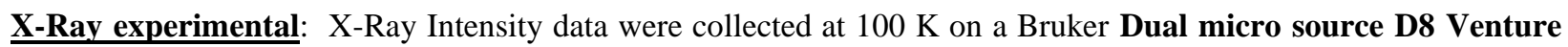
diffractometer and PHOTON III detector running APEX3 software package of programs and using MoK $\square$ radiation $(\lambda=0.71073 \AA)$.

The data frames were integrated and multi-scan scaling was applied in APEX3. Intrinsic phasing structure solution provided all of the non-H atoms.

The structure was refined using full-matrix least-squares refinement (SHELXL, Sheldrick G.M. 2015). The non-H atoms were refined with anisotropic displacement parameters and all of the $\mathrm{H}$ atoms were calculated in idealized positions and refined riding on their parent atoms. The protons on the nitrogen atom are disordered and refined in the two possible positions. In the final cycle of refinement, 6801 reflections (of which 5009 are observed with I > $2 \square(\mathrm{I})$ ) were used to refine 234 parameters and the resulting $\mathrm{R}_{1}, \mathrm{wR}_{2}$ and $\mathrm{S}$ (goodness of fit) were $5.73 \%, 14.64 \%$ and 1.059 , respectively. The refinement was carried out by minimizing the $w_{2}$ function using $\mathrm{F}^{2}$ rather than $\mathrm{F}$ values. $R_{1}$ is calculated to provide a reference to the conventional $R$ value but its function is not minimized.

Sheldrick, G. M. (2015). Acta Cryst. C71, 3-8.

\section{Crystallographic Summary Table for Compound ( \pm )-11}

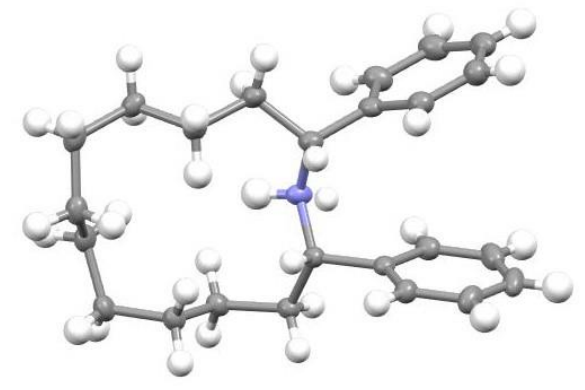

Thermal ellipsoids are drawn at the 50\% probability level.

The requisite CIF has been deposited with the CCDC (deposition \# 2039636).

Table S1. Crystal data and structure refinement for sd200a.

Identification code

Empirical formula

Formula weight

Temperature

Wavelength

Crystal system

Space group

Unit cell dimensions

Volume

Z sd200a

C24 H33 N

335.51

100(2) K

$0.71073 \AA$

Monoclinic

$\mathrm{P} 21 / \mathrm{n}$

$\mathrm{a}=15.9096(4) \AA$

$\mathrm{b}=5.50800(10) \AA$

$\mathrm{c}=23.3293(7) \AA$

1982.09(9) $\AA^{3}$

4 
Table S2. Atomic coordinates ( $\times 10^{4}$ ) and equivalent isotropic displacement parameters $\left(\AA^{2} \times 10^{3}\right.$ ) for sd200a. $U(e q)$ is defined as one third of the trace of the orthogonalized $U^{i j}$ tensor.

\begin{tabular}{|c|c|c|c|c|}
\hline & $\mathrm{x}$ & $\mathrm{y}$ & $\mathrm{z}$ & $\mathrm{U}(\mathrm{eq})$ \\
\hline N1 & $6034(1)$ & $2297(2)$ & $3635(1)$ & $29(1)$ \\
\hline $\mathrm{C} 1$ & $6287(1)$ & $2840(2)$ & $3090(1)$ & $24(1)$ \\
\hline $\mathrm{C} 2$ & $7042(1)$ & $1210(2)$ & $3034(1)$ & $26(1)$ \\
\hline C3 & $7845(1)$ & $1325(2)$ & $3553(1)$ & $27(1)$ \\
\hline $\mathrm{C} 4$ & $8338(1)$ & $3734(2)$ & $3626(1)$ & $28(1)$ \\
\hline $\mathrm{C} 5$ & 9082(1) & $3804(3)$ & $4187(1)$ & $34(1)$ \\
\hline C6 & $8779(1)$ & $4184(2)$ & $4756(1)$ & $31(1)$ \\
\hline C7 & $8535(1)$ & $6822(2)$ & $4842(1)$ & $32(1)$ \\
\hline $\mathrm{C} 8$ & $8006(1)$ & $7224(2)$ & $5302(1)$ & $33(1)$ \\
\hline C9 & $7156(1)$ & $5803(2)$ & $5187(1)$ & $29(1)$ \\
\hline $\mathrm{C} 10$ & $6569(1)$ & $6201(3)$ & $4575(1)$ & $33(1)$ \\
\hline $\mathrm{C} 11$ & $5733(1)$ & $4683(3)$ & $4450(1)$ & $32(1)$ \\
\hline $\mathrm{C} 12$ & $5417(1)$ & $4008(2)$ & $3796(1)$ & $27(1)$ \\
\hline $\mathrm{C} 13$ & $4509(1)$ & $2967(2)$ & $3655(1)$ & $27(1)$ \\
\hline $\mathrm{C} 14$ & $4335(1)$ & $809(2)$ & $3919(1)$ & $35(1)$ \\
\hline $\mathrm{C} 15$ & $3503(1)$ & $-155(2)$ & 3783(1) & $38(1)$ \\
\hline C16 & $2838(1)$ & 1009(3) & $3383(1)$ & $37(1)$ \\
\hline $\mathrm{C} 17$ & $3004(1)$ & $3148(3)$ & $3121(1)$ & $38(1)$ \\
\hline $\mathrm{C} 18$ & $3835(1)$ & $4116(2)$ & $3256(1)$ & $32(1)$ \\
\hline C19 & $5530(1)$ & $2570(2)$ & $2552(1)$ & $24(1)$ \\
\hline $\mathrm{C} 20$ & 4994(1) & $534(2)$ & $2476(1)$ & $31(1)$ \\
\hline $\mathrm{C} 21$ & 4306(1) & $317(3)$ & 1977(1) & $37(1)$ \\
\hline $\mathrm{C} 22$ & $4145(1)$ & $2126(3)$ & $1552(1)$ & $39(1)$ \\
\hline $\mathrm{C} 23$ & $4676(1)$ & $4140(3)$ & $1623(1)$ & $36(1)$ \\
\hline $\mathrm{C} 24$ & $5363(1)$ & $4357(2)$ & 2121(1) & $28(1)$ \\
\hline
\end{tabular}


Table S3. Bond lengths $[\AA]$ and angles $\left[{ }^{\circ}\right]$ for sd200a.

\begin{tabular}{|c|c|}
\hline N1-C1 & $1.4547(14)$ \\
\hline N1-C12 & $1.4738(15)$ \\
\hline N1-H1 & $0.96(2)$ \\
\hline N1-H1' & $1.07(6)$ \\
\hline C1-C19 & $1.5205(15)$ \\
\hline $\mathrm{C} 1-\mathrm{C} 2$ & $1.5315(15)$ \\
\hline C1-H1B & 1.0000 \\
\hline $\mathrm{C} 2-\mathrm{C} 3$ & $1.5302(15)$ \\
\hline $\mathrm{C} 2-\mathrm{H} 2 \mathrm{~A}$ & 0.9900 \\
\hline $\mathrm{C} 2-\mathrm{H} 2 \mathrm{~B}$ & 0.9900 \\
\hline $\mathrm{C} 3-\mathrm{C} 4$ & $1.5293(17)$ \\
\hline C3-H3A & 0.9900 \\
\hline C3-H3B & 0.9900 \\
\hline C4-C5 & $1.5362(16)$ \\
\hline $\mathrm{C} 4-\mathrm{H} 4 \mathrm{~A}$ & 0.9900 \\
\hline C4-H4B & 0.9900 \\
\hline C5-C6 & $1.5342(18)$ \\
\hline C5-H5A & 0.9900 \\
\hline C5-H5B & 0.9900 \\
\hline C6-C7 & $1.5298(18)$ \\
\hline C6-H6A & 0.9900 \\
\hline C6-H6B & 0.9900 \\
\hline C7-C8 & $1.5345(19)$ \\
\hline C7-H7A & 0.9900 \\
\hline C7-H7B & 0.9900 \\
\hline C8-C9 & $1.5282(17)$ \\
\hline C8-H8A & 0.9900 \\
\hline C8-H8B & 0.9900 \\
\hline C9-C10 & $1.5177(16)$ \\
\hline C9-H9A & 0.9900 \\
\hline C9-H9B & 0.9900 \\
\hline C10-C11 & $1.5373(17)$ \\
\hline C10-H10A & 0.9900 \\
\hline C10-H10B & 0.9900 \\
\hline
\end{tabular}




\begin{tabular}{|c|c|}
\hline $\mathrm{C} 11-\mathrm{C} 12$ & $1.5304(16)$ \\
\hline C11-H11A & 0.9900 \\
\hline C11-H11B & 0.9900 \\
\hline C12-C13 & $1.5139(16)$ \\
\hline C12-H12A & 1.0000 \\
\hline C13-C18 & $1.3888(17)$ \\
\hline C13-C14 & $1.3974(18)$ \\
\hline C14-C15 & $1.3891(18)$ \\
\hline C14-H14A & 0.9500 \\
\hline C15-C16 & $1.384(2)$ \\
\hline C15-H15A & 0.9500 \\
\hline $\mathrm{C} 16-\mathrm{C} 17$ & $1.383(2)$ \\
\hline C16-H16A & 0.9500 \\
\hline C17-C18 & $1.3878(18)$ \\
\hline C17-H17A & 0.9500 \\
\hline C18-H18A & 0.9500 \\
\hline C19-C24 & $1.3852(16)$ \\
\hline C19-C20 & $1.3929(16)$ \\
\hline C20-C21 & $1.3942(17)$ \\
\hline $\mathrm{C} 20-\mathrm{H} 20 \mathrm{~A}$ & 0.9500 \\
\hline C21-C22 & $1.384(2)$ \\
\hline $\mathrm{C} 21-\mathrm{H} 21 \mathrm{~A}$ & 0.9500 \\
\hline $\mathrm{C} 22-\mathrm{C} 23$ & $1.379(2)$ \\
\hline $\mathrm{C} 22-\mathrm{H} 22 \mathrm{~A}$ & 0.9500 \\
\hline C23-C24 & $1.3915(16)$ \\
\hline $\mathrm{C} 23-\mathrm{H} 23 \mathrm{~A}$ & 0.9500 \\
\hline $\mathrm{C} 24-\mathrm{H} 24 \mathrm{~A}$ & 0.9500 \\
\hline C1-N1-C12 & $115.83(9)$ \\
\hline C1-N1-H1 & $110.2(14)$ \\
\hline C12-N1-H1 & $111.0(14)$ \\
\hline C1-N1-H1' & $96(3)$ \\
\hline C12-N1-H1' & 119(3) \\
\hline N1-C1-C19 & 111.64(9) \\
\hline $\mathrm{N} 1-\mathrm{C} 1-\mathrm{C} 2$ & $110.25(9)$ \\
\hline $\mathrm{C} 19-\mathrm{C} 1-\mathrm{C} 2$ & $110.55(9)$ \\
\hline
\end{tabular}




\begin{tabular}{|c|c|}
\hline N1-C1-H1B & 108.1 \\
\hline C19-C1-H1B & 108.1 \\
\hline C2-C1-H1B & 108.1 \\
\hline $\mathrm{C} 3-\mathrm{C} 2-\mathrm{C} 1$ & $115.29(9)$ \\
\hline $\mathrm{C} 3-\mathrm{C} 2-\mathrm{H} 2 \mathrm{~A}$ & 108.5 \\
\hline $\mathrm{C} 1-\mathrm{C} 2-\mathrm{H} 2 \mathrm{~A}$ & 108.5 \\
\hline C3-C2-H2B & 108.5 \\
\hline $\mathrm{C} 1-\mathrm{C} 2-\mathrm{H} 2 \mathrm{~B}$ & 108.5 \\
\hline $\mathrm{H} 2 \mathrm{~A}-\mathrm{C} 2-\mathrm{H} 2 \mathrm{~B}$ & 107.5 \\
\hline $\mathrm{C} 4-\mathrm{C} 3-\mathrm{C} 2$ & $115.46(10)$ \\
\hline C4-C3-H3A & 108.4 \\
\hline $\mathrm{C} 2-\mathrm{C} 3-\mathrm{H} 3 \mathrm{~A}$ & 108.4 \\
\hline C4-C3-H3B & 108.4 \\
\hline C2-C3-H3B & 108.4 \\
\hline H3A-C3-H3B & 107.5 \\
\hline $\mathrm{C} 3-\mathrm{C} 4-\mathrm{C} 5$ & $112.59(10)$ \\
\hline C3-C4-H4A & 109.1 \\
\hline C5-C4-H4A & 109.1 \\
\hline C3-C4-H4B & 109.1 \\
\hline C5-C4-H4B & 109.1 \\
\hline $\mathrm{H} 4 \mathrm{~A}-\mathrm{C} 4-\mathrm{H} 4 \mathrm{~B}$ & 107.8 \\
\hline C6-C5-C4 & $113.61(10)$ \\
\hline C6-C5-H5A & 108.8 \\
\hline C4-C5-H5A & 108.8 \\
\hline C6-C5-H5B & 108.8 \\
\hline C4-C5-H5B & 108.8 \\
\hline H5A-C5-H5B & 107.7 \\
\hline C7-C6-C5 & $112.74(11)$ \\
\hline C7-C6-H6A & 109.0 \\
\hline C5-C6-H6A & 109.0 \\
\hline C7-C6-H6B & 109.0 \\
\hline C5-C6-H6B & 109.0 \\
\hline H6A-C6-H6B & 107.8 \\
\hline C6-C7-C8 & $115.40(10)$ \\
\hline C6-C7-H7A & 108.4 \\
\hline C8-C7-H7A & 108.4 \\
\hline
\end{tabular}




\begin{tabular}{|c|c|}
\hline C6-C7-H7B & 108.4 \\
\hline C8-C7-H7B & 108.4 \\
\hline H7A-C7-H7B & 107.5 \\
\hline C9-C8-C7 & $114.63(10)$ \\
\hline C9-C8-H8A & 108.6 \\
\hline C7-C8-H8A & 108.6 \\
\hline C9-C8-H8B & 108.6 \\
\hline C7-C8-H8B & 108.6 \\
\hline H8A-C8-H8B & 107.6 \\
\hline C10-C9-C8 & 113.91(10) \\
\hline C10-C9-H9A & 108.8 \\
\hline C8-C9-H9A & 108.8 \\
\hline С10-С9-H9B & 108.8 \\
\hline C8-C9-H9B & 108.8 \\
\hline H9A-C9-H9B & 107.7 \\
\hline C9-C10-C11 & $113.87(10)$ \\
\hline C9-C10-H10A & 108.8 \\
\hline C11-C10-H10A & 108.8 \\
\hline C9-C10-H10B & 108.8 \\
\hline C11-C10-H10B & 108.8 \\
\hline H10A-C10-H10B & 107.7 \\
\hline C12-C11-C10 & 112.31(10) \\
\hline C12-C11-H11A & 109.1 \\
\hline C10-C11-H11A & 109.1 \\
\hline C12-C11-H11B & 109.1 \\
\hline C10-C11-H11B & 109.1 \\
\hline H11A-C11-H11B & 107.9 \\
\hline N1-C12-C13 & $111.20(10)$ \\
\hline N1-C12-C11 & $109.44(10)$ \\
\hline C13-C12-C11 & $111.85(9)$ \\
\hline N1-C12-H12A & 108.1 \\
\hline C13-C12-H12A & 108.1 \\
\hline C11-C12-H12A & 108.1 \\
\hline C18-C13-C14 & 118.64(11) \\
\hline $\mathrm{C} 18-\mathrm{C} 13-\mathrm{C} 12$ & $120.84(11)$ \\
\hline C14-C13-C12 & $120.51(11)$ \\
\hline
\end{tabular}




\begin{tabular}{ll} 
C15-C14-C13 & $120.29(12)$ \\
C15-C14-H14A & 119.9 \\
C13-C14-H14A & 119.9 \\
C16-C15-C14 & $120.40(13)$ \\
C16-C15-H15A & 119.8 \\
C14-C15-H15A & 119.8 \\
C17-C16-C15 & $119.66(12)$ \\
C17-C16-H16A & 120.2 \\
C15-C16-H16A & 120.2 \\
C16-C17-C18 & $120.12(13)$ \\
C16-C17-H17A & 119.9 \\
C18-C17-H17A & 119.9 \\
C17-C18-C13 & $120.89(12)$ \\
C17-C18-H18A & 119.6 \\
C13-C18-H18A & 119.6 \\
C24-C19-C20 & $118.52(10)$ \\
C24-C19-C1 & $120.15(10)$ \\
C20-C19-C1 & $121.33(10)$ \\
C19-C20-C21 & $120.42(12)$ \\
C19-C20-H20A & 119.8 \\
C21-C20-H20A & 119.8 \\
C22-C21-C20 & $120.33(12)$ \\
C22-C21-H21A & 119.8 \\
C20-C21-H21A & 119.8 \\
C23-C22-C21 & $119.54(12)$ \\
C23-C22-H22A & 120.2 \\
C21-C22-H22A & 120.2 \\
C22-C23-C24 & $120.15(13)$ \\
& 119.9 \\
C24-C23-H23A $24-\mathrm{C} 23$ A & 119.9 \\
C23 & $121.04(12)$ \\
\hline
\end{tabular}


Table S4. Anisotropic displacement parameters $\left(\AA^{2} \mathrm{x} 10^{3}\right)$ for sd200a. The anisotropic displacement factor exponent takes the form: $-2 \pi^{2}\left[h^{2} a^{* 2} U^{11}+\ldots+2 h k a^{*} b^{*} U^{12}\right]$

\begin{tabular}{|c|c|c|c|c|c|c|}
\hline & $\mathrm{U}^{11}$ & $\mathrm{U}^{22}$ & $\mathrm{U}^{33}$ & $\mathrm{U}^{23}$ & $\mathrm{U}^{13}$ & $\mathrm{U}^{12}$ \\
\hline N1 & $29(1)$ & $35(1)$ & $25(1)$ & $6(1)$ & 11(1) & $8(1)$ \\
\hline $\mathrm{C} 1$ & $24(1)$ & $26(1)$ & $22(1)$ & $0(1)$ & $6(1)$ & $2(1)$ \\
\hline $\mathrm{C} 2$ & $24(1)$ & $30(1)$ & $26(1)$ & $-1(1)$ & $8(1)$ & $1(1)$ \\
\hline C3 & $24(1)$ & $31(1)$ & $28(1)$ & $5(1)$ & $7(1)$ & $3(1)$ \\
\hline $\mathrm{C} 4$ & $24(1)$ & $34(1)$ & $26(1)$ & $5(1)$ & $5(1)$ & $0(1)$ \\
\hline C5 & $23(1)$ & $45(1)$ & $30(1)$ & $3(1)$ & $2(1)$ & $-1(1)$ \\
\hline C6 & $28(1)$ & $36(1)$ & $25(1)$ & $5(1)$ & $-1(1)$ & $2(1)$ \\
\hline C7 & $31(1)$ & $32(1)$ & $30(1)$ & $4(1)$ & $0(1)$ & $-5(1)$ \\
\hline $\mathrm{C} 8$ & $38(1)$ & $30(1)$ & $26(1)$ & $-1(1)$ & $0(1)$ & $-2(1)$ \\
\hline C9 & $36(1)$ & $29(1)$ & $22(1)$ & $0(1)$ & $6(1)$ & $0(1)$ \\
\hline $\mathrm{C} 10$ & $31(1)$ & $44(1)$ & $24(1)$ & $4(1)$ & $5(1)$ & $-2(1)$ \\
\hline C11 & $32(1)$ & $41(1)$ & $24(1)$ & $-1(1)$ & $10(1)$ & $-2(1)$ \\
\hline $\mathrm{C} 12$ & $28(1)$ & $28(1)$ & $27(1)$ & $0(1)$ & 11(1) & $2(1)$ \\
\hline $\mathrm{C} 13$ & $30(1)$ & $27(1)$ & $28(1)$ & $-4(1)$ & $13(1)$ & $1(1)$ \\
\hline C14 & $35(1)$ & $31(1)$ & $41(1)$ & $4(1)$ & $15(1)$ & $2(1)$ \\
\hline C15 & $42(1)$ & $30(1)$ & $46(1)$ & $-3(1)$ & $21(1)$ & $-5(1)$ \\
\hline C16 & $35(1)$ & $43(1)$ & $37(1)$ & $-12(1)$ & $13(1)$ & $-8(1)$ \\
\hline $\mathrm{C} 17$ & $33(1)$ & $48(1)$ & $32(1)$ & $-2(1)$ & $7(1)$ & $1(1)$ \\
\hline C18 & $34(1)$ & $33(1)$ & $30(1)$ & $0(1)$ & 11(1) & $1(1)$ \\
\hline C19 & $23(1)$ & $27(1)$ & $22(1)$ & $-3(1)$ & $7(1)$ & $3(1)$ \\
\hline $\mathrm{C} 20$ & $31(1)$ & $30(1)$ & $32(1)$ & $-4(1)$ & 11(1) & $-1(1)$ \\
\hline $\mathrm{C} 21$ & $28(1)$ & $38(1)$ & $46(1)$ & $-16(1)$ & $9(1)$ & $-5(1)$ \\
\hline $\mathrm{C} 22$ & $28(1)$ & $49(1)$ & $36(1)$ & $-16(1)$ & $-2(1)$ & $8(1)$ \\
\hline $\mathrm{C} 23$ & $37(1)$ & $41(1)$ & $27(1)$ & $-1(1)$ & $0(1)$ & 11(1) \\
\hline $\mathrm{C} 24$ & $28(1)$ & $30(1)$ & $26(1)$ & $-1(1)$ & $5(1)$ & $3(1)$ \\
\hline
\end{tabular}


Table S5. Hydrogen coordinates ( x 10 $0^{4}$ ) and isotropic displacement parameters $\left(\AA^{2} \mathrm{x} 10^{3}\right)$ for sd200a.

\begin{tabular}{|c|c|c|c|c|}
\hline & $\mathrm{x}$ & $\mathrm{y}$ & $\mathrm{z}$ & $\mathrm{U}(\mathrm{eq})$ \\
\hline H1 & $6537(15)$ & $2100(40)$ & $3954(11)$ & $48(6)$ \\
\hline $\mathrm{H} 1^{\prime}$ & $5840(40)$ & $470(110)$ & $3520(30)$ & $28(14)$ \\
\hline $\mathrm{H} 1 \mathrm{~B}$ & 6489 & 4564 & 3110 & 29 \\
\hline $\mathrm{H} 2 \mathrm{~A}$ & 6835 & -491 & 2988 & 31 \\
\hline $\mathrm{H} 2 \mathrm{~B}$ & 7216 & 1663 & 2668 & 31 \\
\hline $\mathrm{H} 3 \mathrm{~A}$ & 7663 & 990 & 3922 & 33 \\
\hline $\mathrm{H} 3 \mathrm{~B}$ & 8247 & 16 & 3503 & 33 \\
\hline $\mathrm{H} 4 \mathrm{~A}$ & 7929 & 5073 & 3641 & 34 \\
\hline $\mathrm{H} 4 \mathrm{~B}$ & 8576 & 4002 & 3276 & 34 \\
\hline $\mathrm{H} 5 \mathrm{~A}$ & 9409 & 2259 & 4219 & 40 \\
\hline H5B & 9484 & 5133 & 4149 & 40 \\
\hline H6A & 8270 & 3134 & 4745 & 37 \\
\hline H6B & 9247 & 3678 & 5099 & 37 \\
\hline $\mathrm{H} 7 \mathrm{~A}$ & 8199 & 7460 & 4457 & 39 \\
\hline H7B & 9075 & 7787 & 4960 & 39 \\
\hline $\mathrm{H} 8 \mathrm{~A}$ & 8367 & 6763 & 5696 & 39 \\
\hline H8B & 7873 & 8977 & 5315 & 39 \\
\hline H9A & 7291 & 4050 & 5239 & 35 \\
\hline H9B & 6839 & 6278 & 5486 & 35 \\
\hline H10A & 6895 & 5798 & 4277 & 40 \\
\hline H10B & 6412 & 7941 & 4530 & 40 \\
\hline H11A & 5839 & 3179 & 4689 & 38 \\
\hline H11B & 5274 & 5614 & 4572 & 38 \\
\hline $\mathrm{H} 12 \mathrm{~A}$ & 5407 & 5519 & 3557 & 32 \\
\hline H14A & 4788 & -2 & 4193 & 42 \\
\hline $\mathrm{H} 15 \mathrm{~A}$ & 3390 & -1621 & 3964 & 45 \\
\hline H16A & 2270 & 342 & 3290 & 45 \\
\hline H17A & 2549 & 3956 & 2848 & 46 \\
\hline H18A & 3943 & 5585 & 3073 & 38 \\
\hline H20A & 5099 & -714 & 2766 & 37 \\
\hline
\end{tabular}




\begin{tabular}{lrrrr} 
H21A & 3945 & -1081 & 1928 & 45 \\
H22A & 3673 & 1982 & 1215 & 47 \\
H23A & 4572 & 5381 & 1332 & 44 \\
H24A & 5724 & 5753 & 2166 & 34 \\
\hline
\end{tabular}




\section{Crystallographic Summary for Compound ( \pm )-1m}

X-Ray experimental: X-Ray Intensity data were collected at $100 \mathrm{~K}$ on a Bruker Dual micro source D8 Venture diffractometer and PHOTON III detector running APEX3 software package of programs and using MoK radiation $(\lambda=0.71073 \AA)$.

The data frames were integrated and multi-scan scaling was applied in APEX3. Intrinsic phasing structure solution provided the All of the non-H atoms.

The structure was refined using full-matrix least-squares refinement (SHELXL, Sheldrick G.M. 2015). The non-H atoms were refined with anisotropic displacement parameters and all of the $\mathrm{H}$ atoms were calculated in idealized positions and refined riding on their parent atoms. In the final cycle of refinement, 5263 reflections (of which 4327 are observed with $\mathrm{I}>2 \square(\mathrm{I})$ ) were used to refine 185 parameters and the resulting $\mathrm{R}_{1}$, wR $\mathrm{R}_{2}$ and $\mathrm{S}$ (goodness of fit) were $4.49 \%, 11.46 \%$ and 1.046 , respectively. The refinement was carried out by minimizing the $\mathrm{wR}_{2}$ function using $\mathrm{F}^{2}$ rather than $\mathrm{F}$ values. $\mathrm{R}_{1}$ is calculated to provide a reference to the conventional $\mathrm{R}$ value but its function is not minimized.

Sheldrick, G. M. (2015). Acta Cryst. C71, 3-8.

\section{Crystallographic Summary Table for Compound ( \pm )-1m}

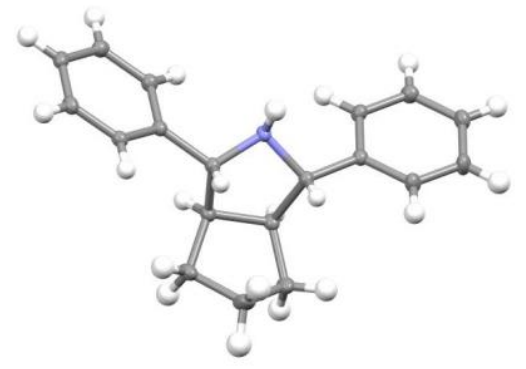

Thermal ellipsoids are drawn at the 50\% probability level.

The requisite CIF has been deposited with the CCDC (deposition \# 2039637).

Table S6. Crystal data and structure refinement for dutta1.

Identification code

Empirical formula

Formula weight

Temperature

Wavelength

Crystal system

Space group

Unit cell dimensions dutta1

C19 H21 N

263.37

100(2) K

$0.71073 \AA$

Monoclinic

$\mathrm{P} 21 / \mathrm{n}$

$\mathrm{a}=5.82460(10) \AA \quad \alpha=90^{\circ}$.

$\mathrm{b}=19.3688(5) \AA \quad \beta=94.5960(10)^{\circ}$.

$\mathrm{c}=13.1245(4) \AA \quad \gamma=90^{\circ}$. 
Volume

Z

Density (calculated)

Absorption coefficient

$\mathrm{F}(000)$

Crystal size

Theta range for data collection

Index ranges

Reflections collected

Independent reflections

Completeness to theta $=25.242^{\circ}$

Absorption correction

Max. and min. transmission

Refinement method

Data / restraints / parameters

Goodness-of-fit on $\mathrm{F}^{2}$

Final R indices [I>2sigma(I)]

$\mathrm{R}$ indices (all data)

Extinction coefficient

Largest diff. peak and hole
1475.89(6) $\AA^{3}$

4

$1.185 \mathrm{Mg} / \mathrm{m}^{3}$

$0.068 \mathrm{~mm}^{-1}$

568

$0.216 \times 0.216 \times 0.050 \mathrm{~mm}^{3}$

1.878 to $33.295^{\circ}$.

$-6 \leq \mathrm{h} \leq 8,-29 \leq \mathrm{k} \leq 29,-19 \leq \mathrm{l} \leq 20$

52218

$5263[\mathrm{R}(\mathrm{int})=0.0513]$

$99.7 \%$

Semi-empirical from equivalents

0.9966 and 0.9884

Full-matrix least-squares on $\mathrm{F}^{2}$

5263 / 0 / 185

1.046

$\mathrm{R} 1=0.0449, \mathrm{wR} 2=0.1146$ [4327]

$\mathrm{R} 1=0.0565, \mathrm{wR} 2=0.1227$

$\mathrm{n} / \mathrm{a}$

0.388 and -0.200 e. $\AA^{-3}$

$R 1=\sum\left(|| F_{O}|-| F_{C}||\right) / \sum\left|F_{O}\right| \quad w R 2=\left[\sum\left[w\left(F_{O}{ }^{2}-F_{C}{ }^{2}\right)^{2}\right] / \sum\left[w\left(F_{O}{ }^{2}\right)^{2}\right]\right]^{1 / 2}$

$S=\left[\sum\left[w\left(F_{O}{ }^{2}-F_{C}{ }^{2}\right)^{2}\right] /(n-p)\right]^{1 / 2} w=1 /\left[\sigma^{2}\left(F_{O}{ }^{2}\right)+\left(m^{*} p\right)^{2}+n^{*} p\right], p=\left[\max \left(F_{O}{ }^{2}, 0\right)+2^{*} F_{C}{ }^{2}\right] / 3$, $m$ \& $n$ are constants. 
Table S7. Atomic coordinates ( $\times 10^{4}$ ) and equivalent isotropic displacement parameters $\left(\AA^{2} \times 10^{3}\right)$ for dutta1. $\mathrm{U}(\mathrm{eq})$ is defined as one third of the trace of the orthogonalized $\mathrm{U}^{\mathrm{ij}}$ tensor.

\begin{tabular}{|c|c|c|c|c|}
\hline & $\mathrm{x}$ & $\mathrm{y}$ & $\mathrm{z}$ & $\mathrm{U}(\mathrm{eq})$ \\
\hline $\mathrm{N} 1$ & $5053(1)$ & 4943(1) & $3232(1)$ & $17(1)$ \\
\hline $\mathrm{C} 1$ & $3932(1)$ & $4802(1)$ & $2212(1)$ & $16(1)$ \\
\hline $\mathrm{C} 3$ & $3010(2)$ & $5682(1)$ & $757(1)$ & $25(1)$ \\
\hline $\mathrm{C} 2$ & $4810(2)$ & $5403(1)$ & 1573(1) & $18(1)$ \\
\hline $\mathrm{C} 4$ & $1767(2)$ & $6248(1)$ & $1312(1)$ & $30(1)$ \\
\hline $\mathrm{C} 5$ & $3718(2)$ & $6600(1)$ & 1969(1) & $26(1)$ \\
\hline C6 & $5270(2)$ & 5999(1) & $2363(1)$ & $18(1)$ \\
\hline $\mathrm{C} 8$ & $5941(1)$ & $5971(1)$ & $4322(1)$ & $16(1)$ \\
\hline $\mathrm{C} 7$ & $4624(1)$ & $5682(1)$ & $3384(1)$ & $16(1)$ \\
\hline $\mathrm{C} 12$ & $9354(2)$ & $6022(1)$ & $5503(1)$ & $21(1)$ \\
\hline $\mathrm{C} 11$ & $8341(2)$ & $6529(1)$ & $6070(1)$ & $21(1)$ \\
\hline $\mathrm{C} 10$ & $6138(2)$ & $6760(1)$ & $5765(1)$ & $20(1)$ \\
\hline C9 & $4946(2)$ & 6481(1) & $4898(1)$ & $17(1)$ \\
\hline $\mathrm{C} 14$ & $4440(1)$ & 4093(1) & 1811(1) & $16(1)$ \\
\hline $\mathrm{C} 13$ & $8169(2)$ & $5748(1)$ & $4630(1)$ & $18(1)$ \\
\hline $\mathrm{C} 15$ & $6585(2)$ & $3783(1)$ & $2015(1)$ & $19(1)$ \\
\hline $\mathrm{C} 16$ & $7019(2)$ & $3129(1)$ & 1631(1) & $21(1)$ \\
\hline $\mathrm{C} 17$ & $5326(2)$ & 2781(1) & $1025(1)$ & $23(1)$ \\
\hline C18 & $3200(2)$ & $3093(1)$ & $808(1)$ & $25(1)$ \\
\hline C19 & $2759(2)$ & $3742(1)$ & 1201(1) & $22(1)$ \\
\hline
\end{tabular}


Table S8. Bond lengths $[\AA]$ and angles $\left[{ }^{\circ}\right]$ for dutta 1 .

\begin{tabular}{|c|c|}
\hline $\mathrm{N} 1-\mathrm{C} 1$ & $1.4676(11)$ \\
\hline $\mathrm{N} 1-\mathrm{C} 7$ & $1.4685(10)$ \\
\hline N1-H1 & $0.892(13)$ \\
\hline $\mathrm{C} 1-\mathrm{C} 14$ & $1.5078(11)$ \\
\hline $\mathrm{C} 1-\mathrm{C} 2$ & $1.5466(11)$ \\
\hline C1-H1A & 1.0000 \\
\hline $\mathrm{C} 3-\mathrm{C} 4$ & $1.5299(14)$ \\
\hline $\mathrm{C} 3-\mathrm{C} 2$ & $1.5354(12)$ \\
\hline C3-H3A & 0.9900 \\
\hline C3-H3B & 0.9900 \\
\hline $\mathrm{C} 2-\mathrm{C} 6$ & $1.5596(11)$ \\
\hline $\mathrm{C} 2-\mathrm{H} 2 \mathrm{~A}$ & 1.0000 \\
\hline $\mathrm{C} 4-\mathrm{C} 5$ & $1.5298(15)$ \\
\hline C4-H4A & 0.9900 \\
\hline C4-H4B & 0.9900 \\
\hline C5-C6 & $1.5376(12)$ \\
\hline C5-H5A & 0.9900 \\
\hline C5-H5B & 0.9900 \\
\hline C6-C7 & $1.5478(11)$ \\
\hline C6-H6A & 1.0000 \\
\hline $\mathrm{C} 8-\mathrm{C} 9$ & $1.3971(11)$ \\
\hline C8-C13 & $1.3973(12)$ \\
\hline $\mathrm{C} 8-\mathrm{C} 7$ & $1.5060(11)$ \\
\hline C7-H7A & 1.0000 \\
\hline C12-C11 & $1.3922(13)$ \\
\hline $\mathrm{C} 12-\mathrm{C} 13$ & $1.3940(12)$ \\
\hline C12-H12A & 0.9500 \\
\hline C11-C10 & $1.3866(13)$ \\
\hline C11-H11A & 0.9500 \\
\hline C10-C9 & $1.3928(12)$ \\
\hline C10-H10A & 0.9500 \\
\hline C9-H9A & 0.9500 \\
\hline C14-C19 & $1.3910(11)$ \\
\hline C14-C15 & $1.3930(12)$ \\
\hline
\end{tabular}




\begin{tabular}{|c|c|}
\hline C13-H13A & 0.9500 \\
\hline $\mathrm{C} 15-\mathrm{C} 16$ & $1.3927(12)$ \\
\hline C15-H15A & 0.9500 \\
\hline $\mathrm{C} 16-\mathrm{C} 17$ & $1.3904(12)$ \\
\hline C16-H16A & 0.9500 \\
\hline $\mathrm{C} 17-\mathrm{C} 18$ & $1.3871(13)$ \\
\hline C17-H17A & 0.9500 \\
\hline C18-C19 & $1.3915(12)$ \\
\hline C18-H18A & 0.9500 \\
\hline C19-H19A & 0.9500 \\
\hline C1-N1-C7 & $103.72(6)$ \\
\hline C1-N1-H1 & $111.8(8)$ \\
\hline C7-N1-H1 & $109.6(8)$ \\
\hline N1-C1-C14 & $113.75(7)$ \\
\hline $\mathrm{N} 1-\mathrm{C} 1-\mathrm{C} 2$ & $102.26(6)$ \\
\hline C14-C1-C2 & $114.47(7)$ \\
\hline N1-C1-H1A & 108.7 \\
\hline C14-C1-H1A & 108.7 \\
\hline C2-C1-H1A & 108.7 \\
\hline C4-C3-C2 & $104.03(7)$ \\
\hline C4-C3-H3A & 110.9 \\
\hline $\mathrm{C} 2-\mathrm{C} 3-\mathrm{H} 3 \mathrm{~A}$ & 110.9 \\
\hline C4-C3-H3B & 110.9 \\
\hline C2-C3-H3B & 110.9 \\
\hline H3A-C3-H3B & 109.0 \\
\hline C3-C2-C1 & $114.01(7)$ \\
\hline C3-C2-C6 & $105.96(7)$ \\
\hline C1-C2-C6 & 104.07(6) \\
\hline $\mathrm{C} 3-\mathrm{C} 2-\mathrm{H} 2 \mathrm{~A}$ & 110.8 \\
\hline $\mathrm{C} 1-\mathrm{C} 2-\mathrm{H} 2 \mathrm{~A}$ & 110.8 \\
\hline $\mathrm{C} 6-\mathrm{C} 2-\mathrm{H} 2 \mathrm{~A}$ & 110.8 \\
\hline C5-C4-C3 & $103.24(8)$ \\
\hline C5-C4-H4A & 111.1 \\
\hline $\mathrm{C} 3-\mathrm{C} 4-\mathrm{H} 4 \mathrm{~A}$ & 111.1 \\
\hline C5-C4-H4B & 111.1 \\
\hline
\end{tabular}




\begin{tabular}{|c|c|}
\hline C3-C4-H4B & 111.1 \\
\hline H4A-C4-H4B & 109.1 \\
\hline C4-C5-C6 & $104.05(7)$ \\
\hline C4-C5-H5A & 110.9 \\
\hline C6-C5-H5A & 110.9 \\
\hline C4-C5-H5B & 110.9 \\
\hline C6-C5-H5B & 110.9 \\
\hline H5A-C5-H5B & 109.0 \\
\hline C5-C6-C7 & $114.45(7)$ \\
\hline C5-C6-C2 & $105.73(7)$ \\
\hline C7-C6-C2 & $103.97(6)$ \\
\hline C5-C6-H6A & 110.8 \\
\hline C7-C6-H6A & 110.8 \\
\hline C2-C6-H6A & 110.8 \\
\hline C9-C8-C13 & $118.55(8)$ \\
\hline C9-C8-C7 & $119.99(7)$ \\
\hline $\mathrm{C} 13-\mathrm{C} 8-\mathrm{C} 7$ & $121.45(7)$ \\
\hline N1-C7-C8 & $113.12(7)$ \\
\hline N1-C7-C6 & $102.33(6)$ \\
\hline C8-C7-C6 & $114.62(7)$ \\
\hline N1-C7-H7A & 108.8 \\
\hline C8-C7-H7A & 108.8 \\
\hline C6-C7-H7A & 108.8 \\
\hline C11-C12-C13 & $120.33(8)$ \\
\hline $\mathrm{C} 11-\mathrm{C} 12-\mathrm{H} 12 \mathrm{~A}$ & 119.8 \\
\hline C13-C12-H12A & 119.8 \\
\hline C10-C11-C12 & $119.65(8)$ \\
\hline C10-C11-H11A & 120.2 \\
\hline C12-C11-H11A & 120.2 \\
\hline C11-C10-C9 & $120.02(8)$ \\
\hline C11-C10-H10A & 120.0 \\
\hline C9-C10-H10A & 120.0 \\
\hline C10-C9-C8 & $120.98(8)$ \\
\hline C10-С9-H9A & 119.5 \\
\hline C8-C9-H9A & 119.5 \\
\hline C19-C14-C15 & $118.62(8)$ \\
\hline
\end{tabular}




$\begin{array}{ll}\text { C19-C14-C1 } & 119.78(7) \\ \text { C15-C14-C1 } & 121.57(7) \\ \text { C12-C13-C8 } & 120.46(8) \\ \text { C12-C13-H13A } & 119.8 \\ \text { C8-C13-H13A } & 119.8 \\ \text { C16-C15-C14 } & 120.54(8) \\ \text { C16-C15-H15A } & 119.7 \\ \text { C14-C15-H15A } & 119.7 \\ \text { C17-C16-C15 } & 120.50(8) \\ \text { C17-C16-H16A } & 119.8 \\ \text { C15-C16-H16A } & 119.8 \\ \text { C18-C17-C16 } & 119.11(8) \\ \text { C18-C17-H17A } & 120.4 \\ \text { C16-C17-H17A } & 120.4 \\ \text { C17-C18-C19 } & 120.38(8) \\ \text { C17-C18-H18A } & 119.8 \\ \text { C19-C18-H18A } & 119.8 \\ \text { C14-C19-C18 } & 120.85(8) \\ \text { C14-C19-H19A } & 119.6 \\ \text { C18-C19-H19A } & 119.6\end{array}$


Table S9. Anisotropic displacement parameters $\left(\AA^{2} \times 10^{3}\right)$ for dutta1. The anisotropic displacement factor exponent takes the form: $-2 \pi^{2}\left[h^{2} a^{* 2} U^{11}+\ldots+2 h k a^{*} b^{*} U^{12}\right]$

\begin{tabular}{|c|c|c|c|c|c|c|}
\hline & $\mathrm{U}^{11}$ & $\mathrm{U}^{22}$ & $\mathrm{U}^{33}$ & $\mathrm{U}^{23}$ & $\mathrm{U}^{13}$ & $\mathrm{U}^{12}$ \\
\hline N1 & $23(1)$ & $13(1)$ & $14(1)$ & $0(1)$ & $0(1)$ & $-2(1)$ \\
\hline $\mathrm{C} 1$ & $18(1)$ & $15(1)$ & $15(1)$ & $0(1)$ & $0(1)$ & $-1(1)$ \\
\hline C3 & $38(1)$ & $19(1)$ & $17(1)$ & 1(1) & $-4(1)$ & $1(1)$ \\
\hline $\mathrm{C} 2$ & $23(1)$ & $16(1)$ & $15(1)$ & $0(1)$ & $3(1)$ & $-1(1)$ \\
\hline $\mathrm{C} 4$ & $38(1)$ & $26(1)$ & $26(1)$ & 1(1) & $-9(1)$ & 11(1) \\
\hline C5 & $42(1)$ & $16(1)$ & $20(1)$ & $1(1)$ & $-2(1)$ & $5(1)$ \\
\hline C6 & $22(1)$ & $14(1)$ & $16(1)$ & 1(1) & $2(1)$ & $-1(1)$ \\
\hline $\mathrm{C} 8$ & $18(1)$ & $14(1)$ & $15(1)$ & $1(1)$ & $1(1)$ & $-1(1)$ \\
\hline C7 & $17(1)$ & $14(1)$ & $16(1)$ & $0(1)$ & $1(1)$ & $0(1)$ \\
\hline C12 & $20(1)$ & $19(1)$ & $22(1)$ & $2(1)$ & $-2(1)$ & $-2(1)$ \\
\hline C11 & $28(1)$ & $17(1)$ & $17(1)$ & 1(1) & $-2(1)$ & $-4(1)$ \\
\hline C10 & $28(1)$ & $15(1)$ & $18(1)$ & $-1(1)$ & $2(1)$ & $0(1)$ \\
\hline C9 & $20(1)$ & $14(1)$ & $18(1)$ & $1(1)$ & $2(1)$ & $1(1)$ \\
\hline C14 & $19(1)$ & $15(1)$ & $15(1)$ & $0(1)$ & $1(1)$ & $-2(1)$ \\
\hline $\mathrm{C} 13$ & $18(1)$ & $17(1)$ & $20(1)$ & $-1(1)$ & $1(1)$ & $0(1)$ \\
\hline C15 & $21(1)$ & $19(1)$ & $18(1)$ & $-2(1)$ & $-2(1)$ & $0(1)$ \\
\hline C16 & $22(1)$ & $20(1)$ & $21(1)$ & $-2(1)$ & $0(1)$ & $3(1)$ \\
\hline $\mathrm{C} 17$ & $27(1)$ & $16(1)$ & $24(1)$ & $-3(1)$ & $0(1)$ & $0(1)$ \\
\hline C18 & $26(1)$ & $18(1)$ & $31(1)$ & $-6(1)$ & $-6(1)$ & $-3(1)$ \\
\hline C19 & $19(1)$ & $18(1)$ & $28(1)$ & $-2(1)$ & $-2(1)$ & $-2(1)$ \\
\hline
\end{tabular}


Table S10. Hydrogen coordinates ( $\times 10^{4}$ ) and isotropic displacement parameters $\left(\AA^{2} \times 10^{3}\right)$ for dutta1.

\begin{tabular}{|c|c|c|c|c|}
\hline & $\mathrm{x}$ & $\mathrm{y}$ & $\mathrm{z}$ & $\mathrm{U}(\mathrm{eq})$ \\
\hline $\mathrm{H} 1$ & $4420(20)$ & $4699(7)$ & $3715(10)$ & $27(3)$ \\
\hline $\mathrm{H} 1 \mathrm{~A}$ & 2229 & 4850 & 2239 & 19 \\
\hline $\mathrm{H} 3 \mathrm{~A}$ & 3760 & 5874 & 168 & 30 \\
\hline H3B & 1924 & 5314 & 510 & 30 \\
\hline $\mathrm{H} 2 \mathrm{~A}$ & 6258 & 5271 & 1261 & 21 \\
\hline $\mathrm{H} 4 \mathrm{~A}$ & 991 & 6578 & 821 & 37 \\
\hline $\mathrm{H} 4 \mathrm{~B}$ & 613 & 6049 & 1741 & 37 \\
\hline H5A & 3112 & 6854 & 2544 & 31 \\
\hline H5B & 4571 & 6925 & 1557 & 31 \\
\hline H6A & 6928 & 6138 & 2410 & 21 \\
\hline $\mathrm{H} 7 \mathrm{~A}$ & 2940 & 5752 & 3444 & 19 \\
\hline $\mathrm{H} 12 \mathrm{~A}$ & 10860 & 5861 & 5712 & 25 \\
\hline H11A & 9155 & 6717 & 6662 & 25 \\
\hline $\mathrm{H} 10 \mathrm{~A}$ & 5442 & 7108 & 6147 & 24 \\
\hline H9A & 3435 & 6640 & 4696 & 21 \\
\hline $\mathrm{H} 13 \mathrm{~A}$ & 8882 & 5406 & 4243 & 22 \\
\hline $\mathrm{H} 15 \mathrm{~A}$ & 7762 & 4019 & 2420 & 23 \\
\hline H16A & 8482 & 2921 & 1783 & 25 \\
\hline H17A & 5622 & 2335 & 764 & 27 \\
\hline $\mathrm{H} 18 \mathrm{~A}$ & 2039 & 2862 & 387 & 30 \\
\hline H19A & 1291 & 3949 & 1052 & 26 \\
\hline
\end{tabular}




\section{References}

1. Suffert, J. Simple Direct Titration of Organolithium Reagents Using $N$-Pivaloyl-o-toluidine and/or N-Pivaloyl -o-benzylaniline. J. Org. Chem. 1989, 54, 509.

2. Furber, M.; Herbert, J. M.; Taylor, R. J. K. Stereoselective synthesis of $2 Z, 4 E$-dienals by addition of organometallic reagents to pyrylium perchlorate. J. Chem. Soc. Perkin Trans. I 1989, 683.

3. Chen, W. J.; Ma, L. L.; Paul, A.; Seidel, D. Direct $\alpha-\mathrm{C}-\mathrm{H}$ bond functionalization of unprotected cyclic amines. Nat. Chem. 2018, 10, 165. 
${ }^{1} \mathrm{H}-\mathrm{NMR}$ of $( \pm)-1 \mathbf{a}\left(400 \mathrm{MHz}, \mathrm{CDCl}_{3}\right)$ at $25^{\circ} \mathrm{C}$<smiles>c1ccc([C@@H]2CC[C@H](c3ccccc3)N2)cc1</smiles>

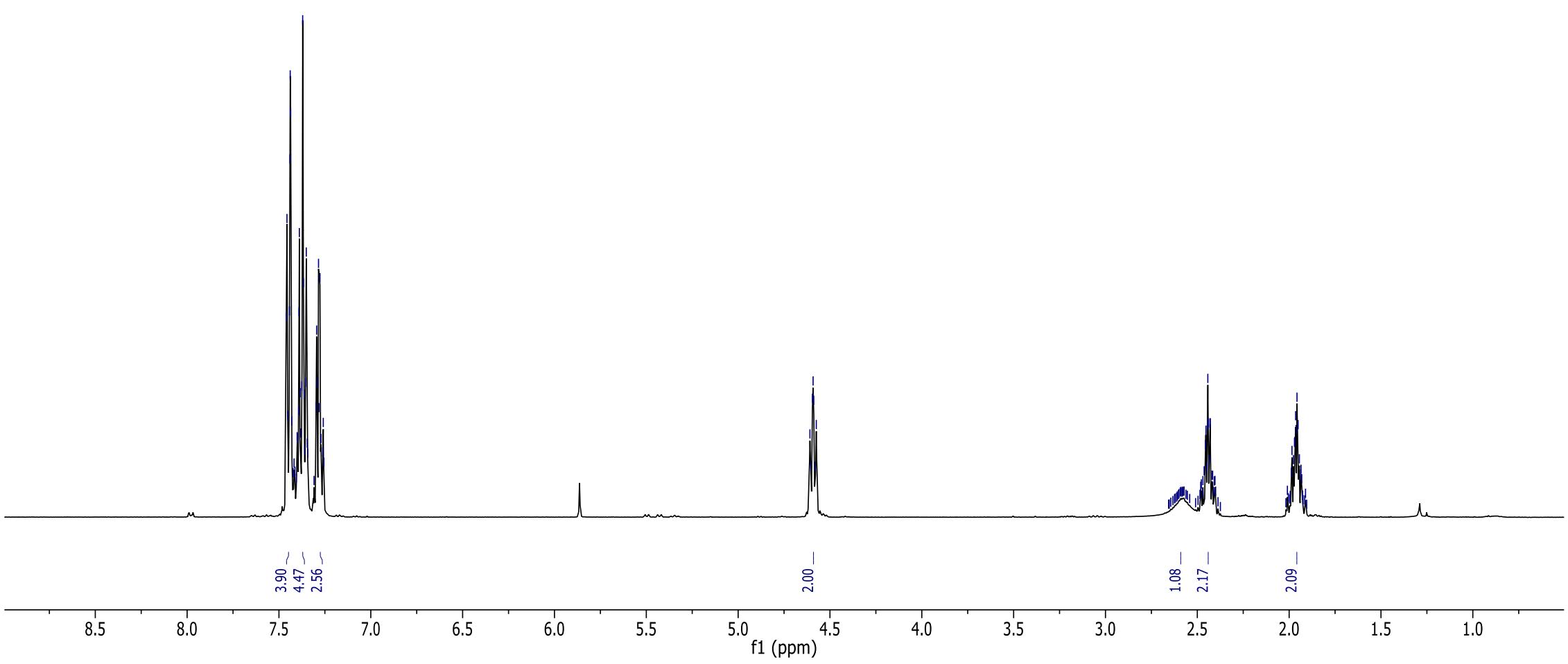




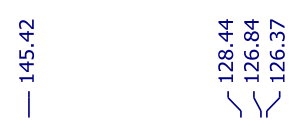

${ }^{13} \mathrm{C}-\mathrm{NMR}$ of $( \pm)-\mathbf{1 a}\left(100 \mathrm{MHz}, \mathrm{CDCl}_{3}\right)$ at $25^{\circ} \mathrm{C}$

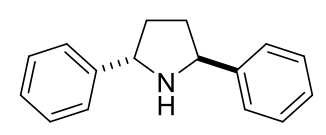

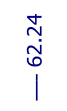

m m
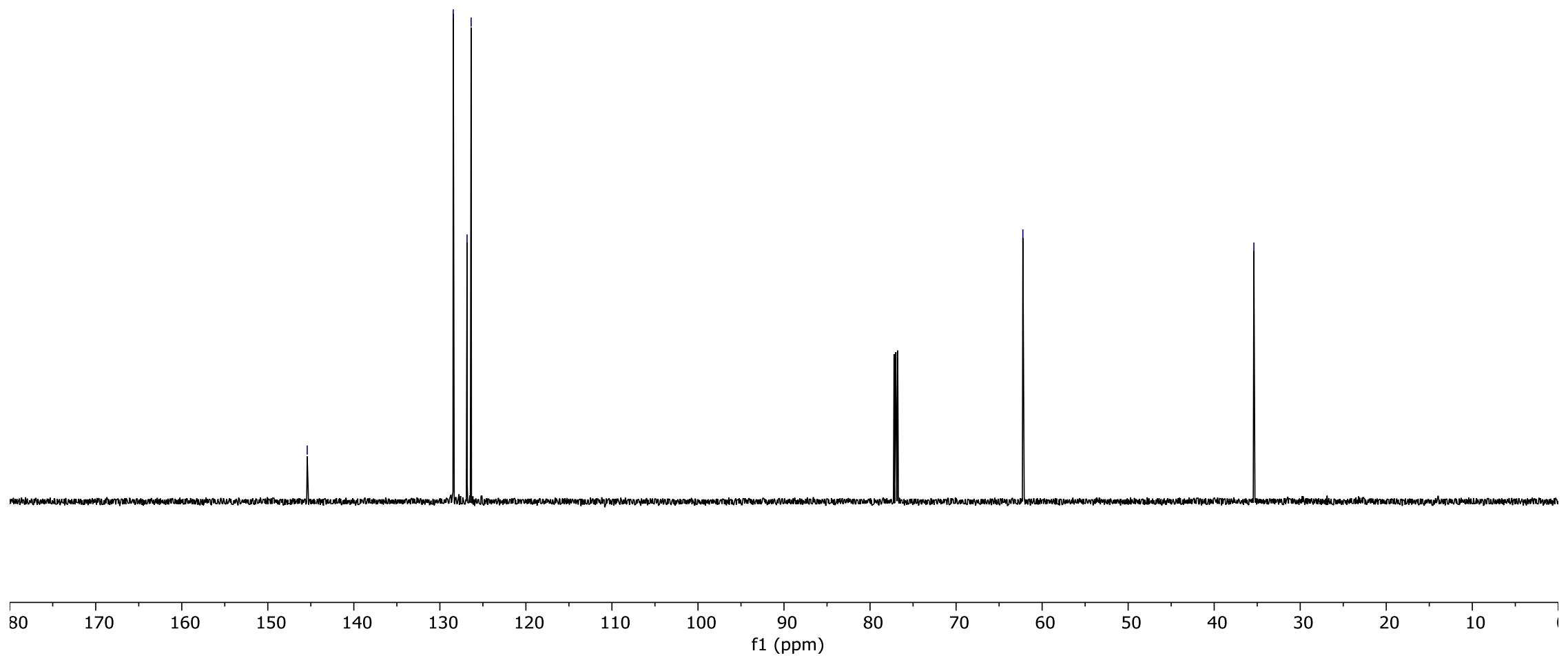

S-74 


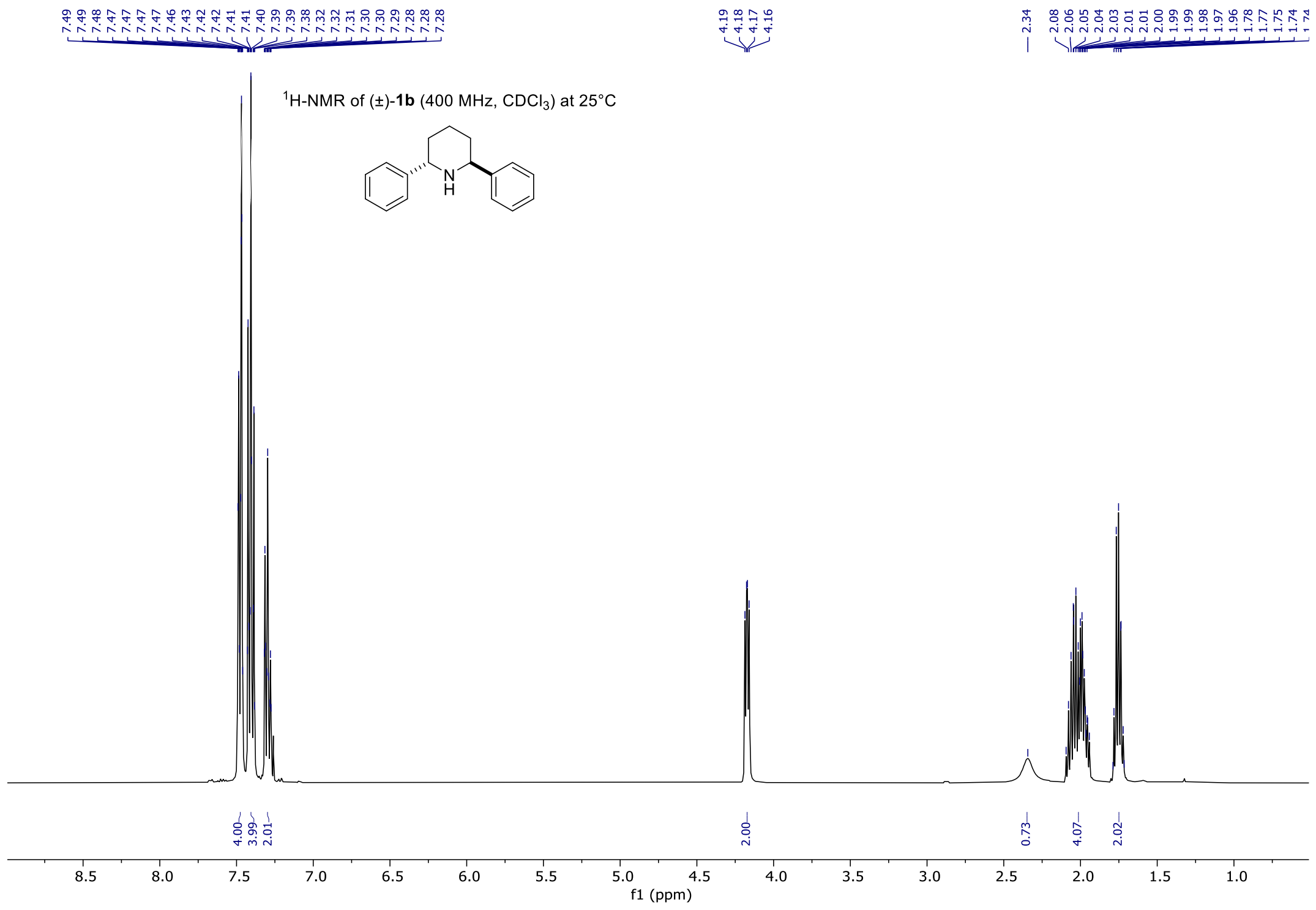



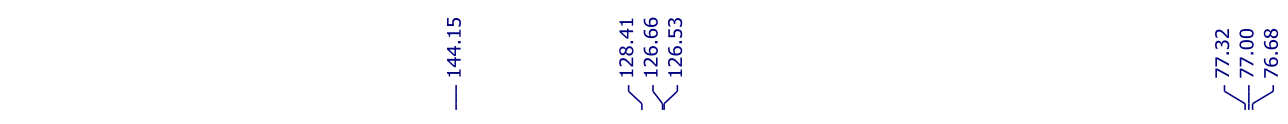

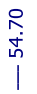

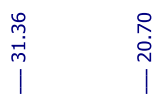

${ }^{13} \mathrm{C}-\mathrm{NMR}$ of $( \pm)-\mathbf{1 b}\left(100 \mathrm{MHz}, \mathrm{CDCl}_{3}\right)$ at $25^{\circ} \mathrm{C}$
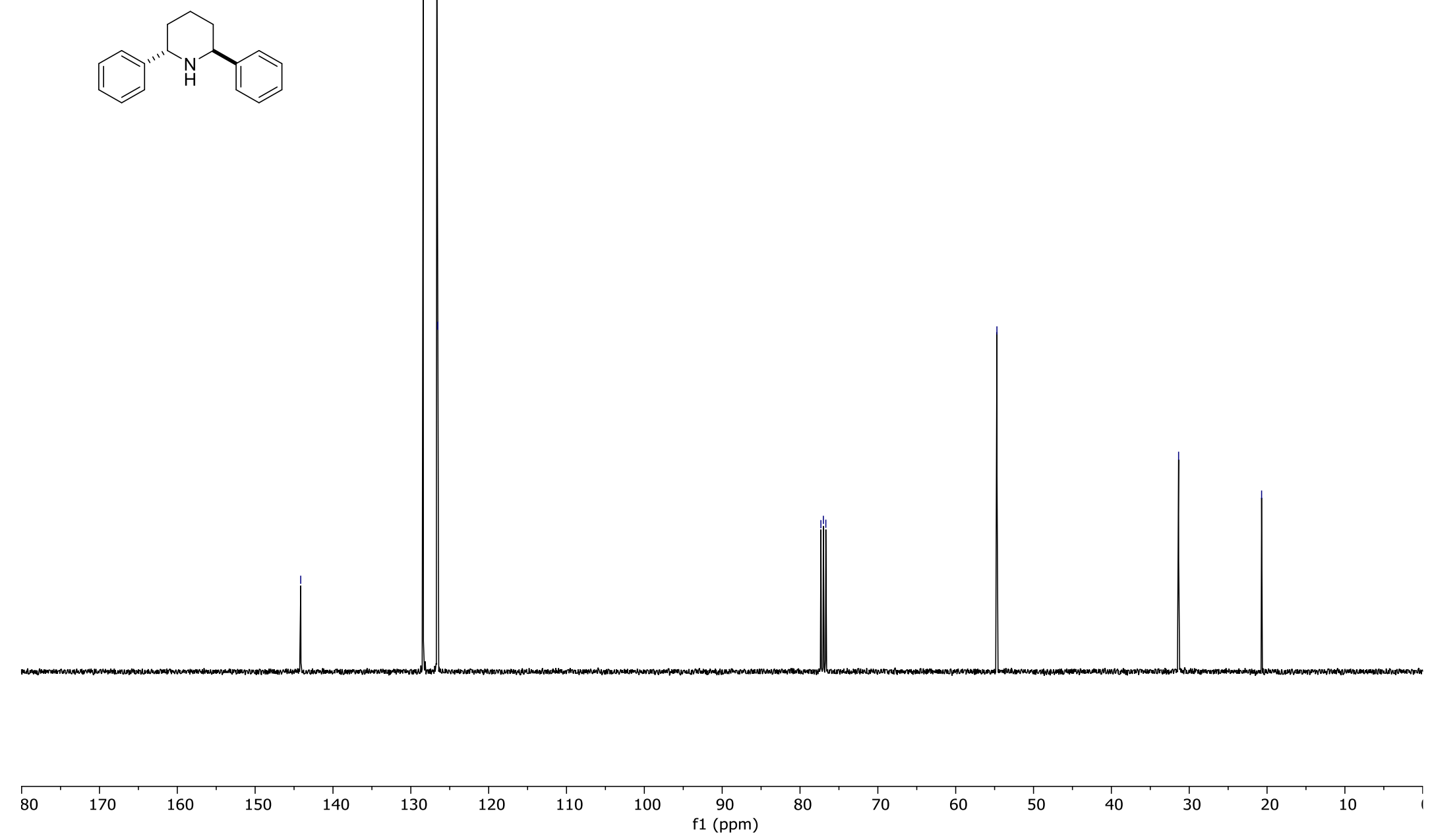

S-76 


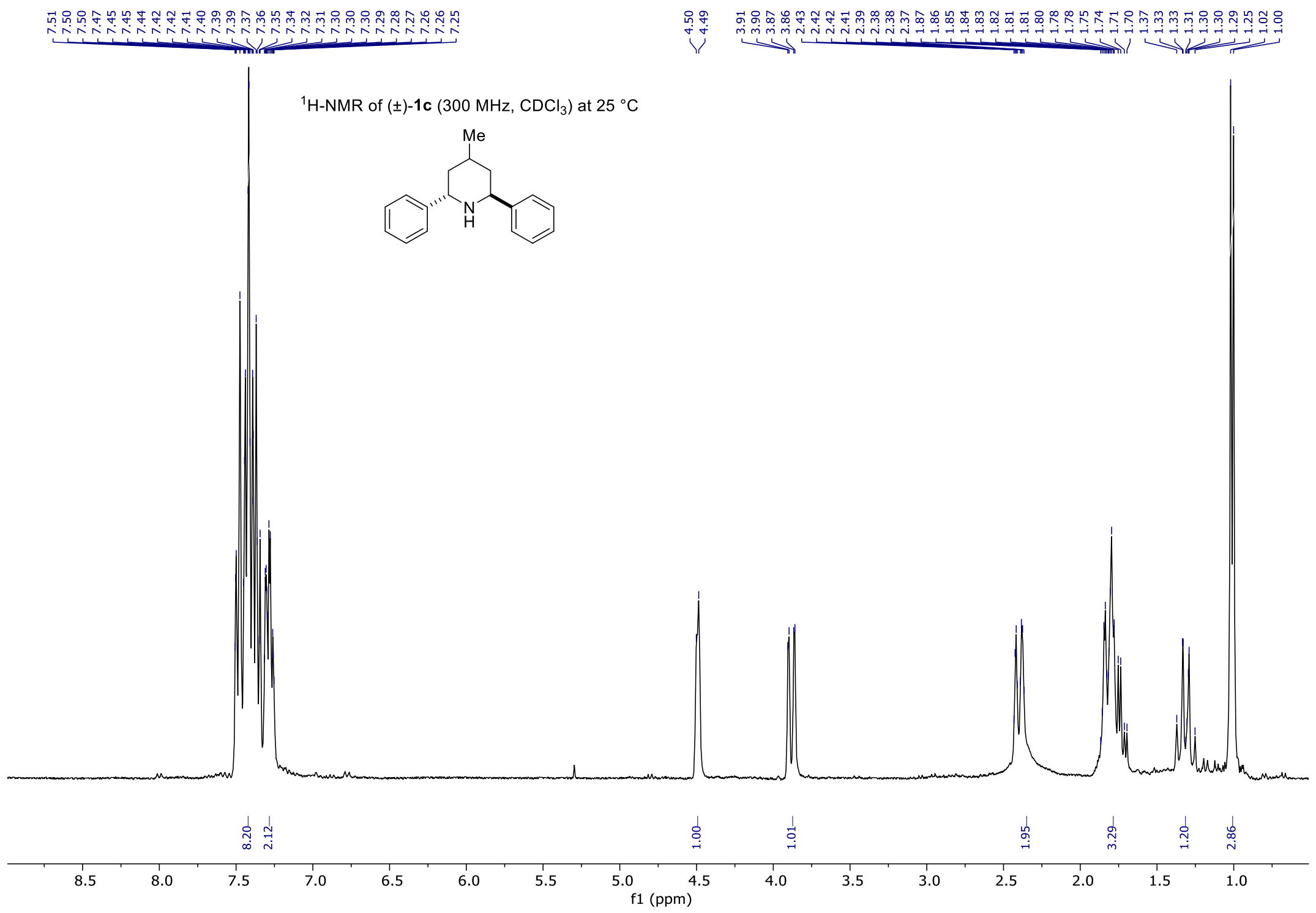




$$
\text { 年 }
$$

${ }^{13} \mathrm{C}-N M R$ of $( \pm)-1 \mathrm{C}\left(75 \mathrm{MHz}, \mathrm{CDCl}_{3}\right)$ at $25^{\circ} \mathrm{C}$<smiles>CC1CC(c2ccccc2)N[C@H](c2ccccc2)C1</smiles>

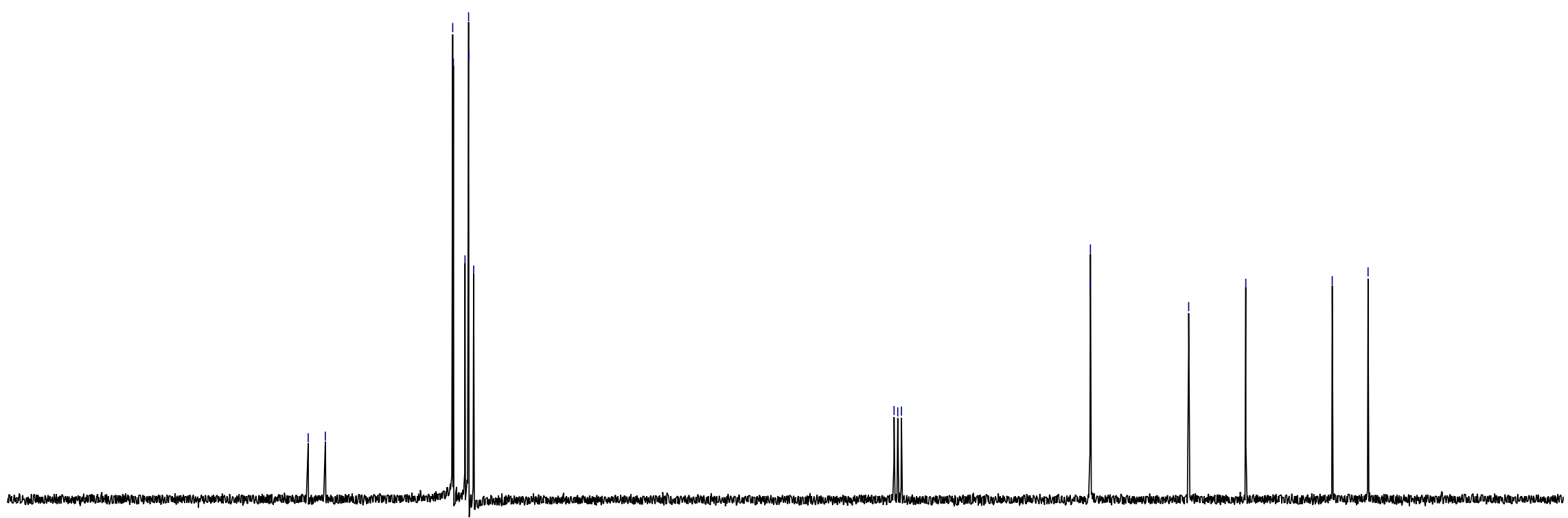

80

150

140

$130 \quad 120$

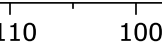

10090

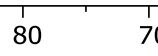

$70 \quad 60$

50

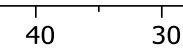

$30 \quad 20$ 


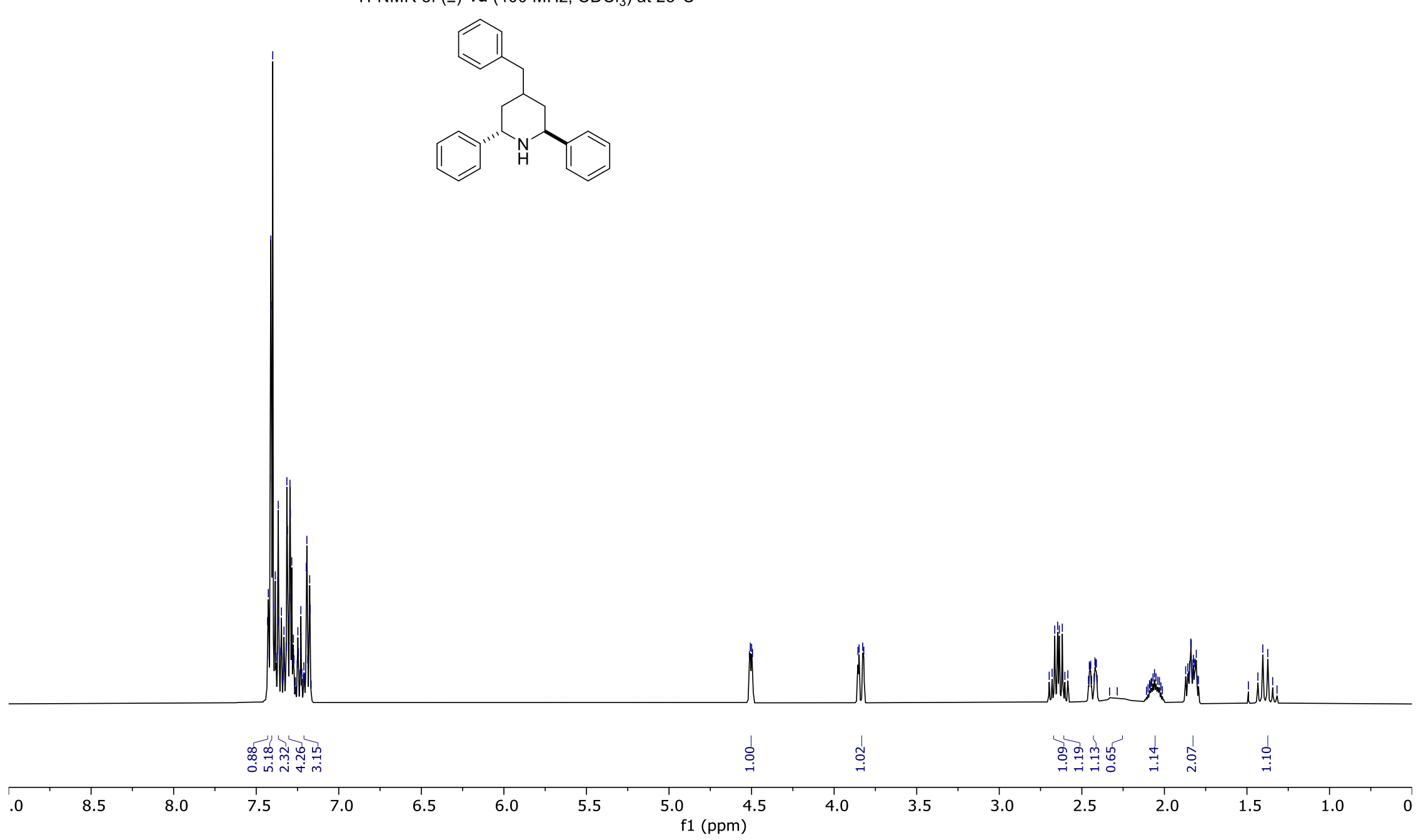




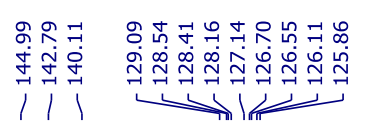

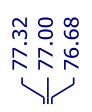

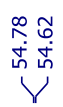

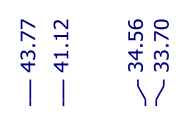

${ }^{13} \mathrm{C}-\mathrm{NMR}$ of $( \pm)-\mathbf{1 d}\left(100 \mathrm{MHz}, \mathrm{CDCl}_{3}\right)$ at $25^{\circ} \mathrm{C}$
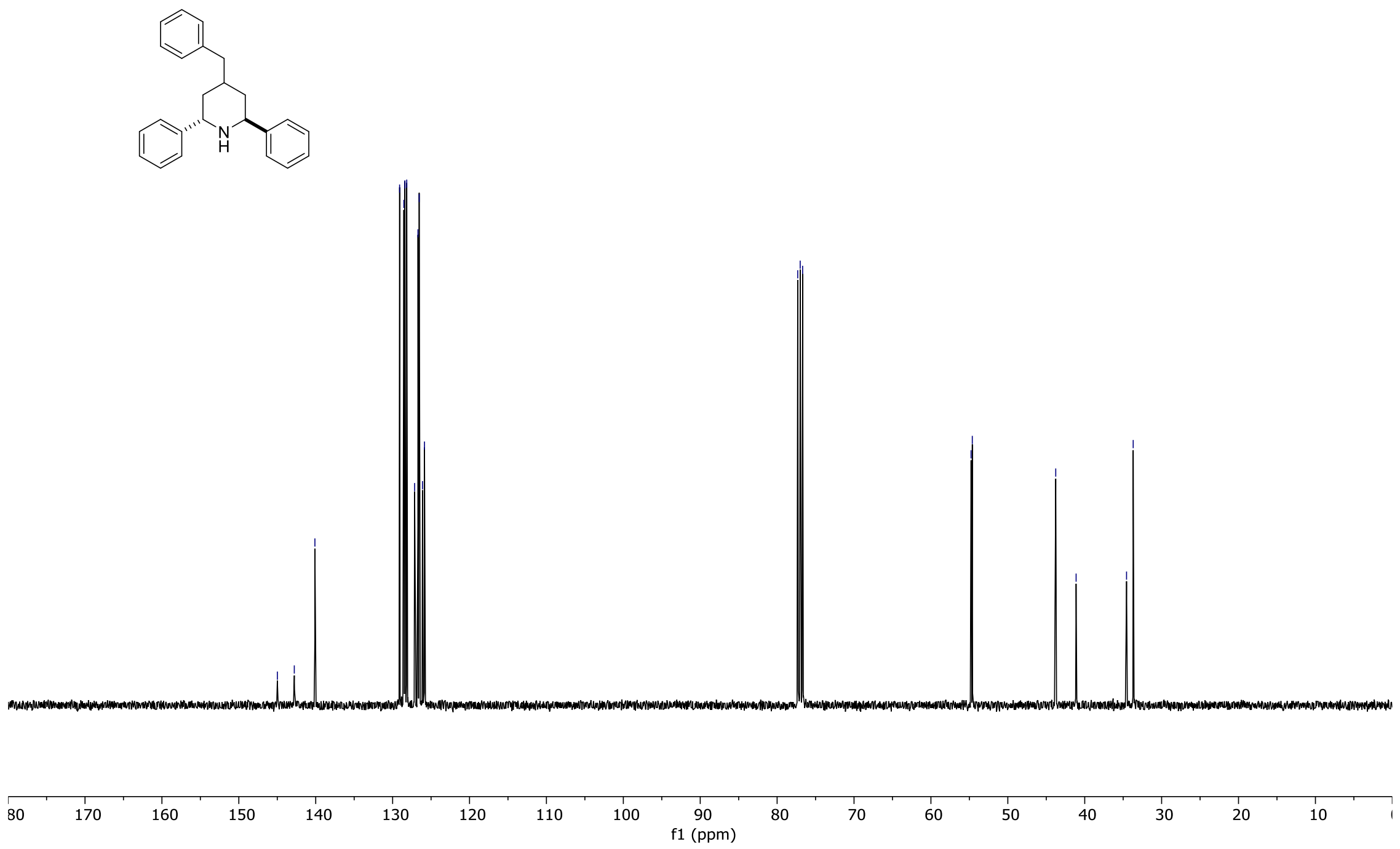

S-80 


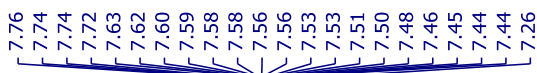
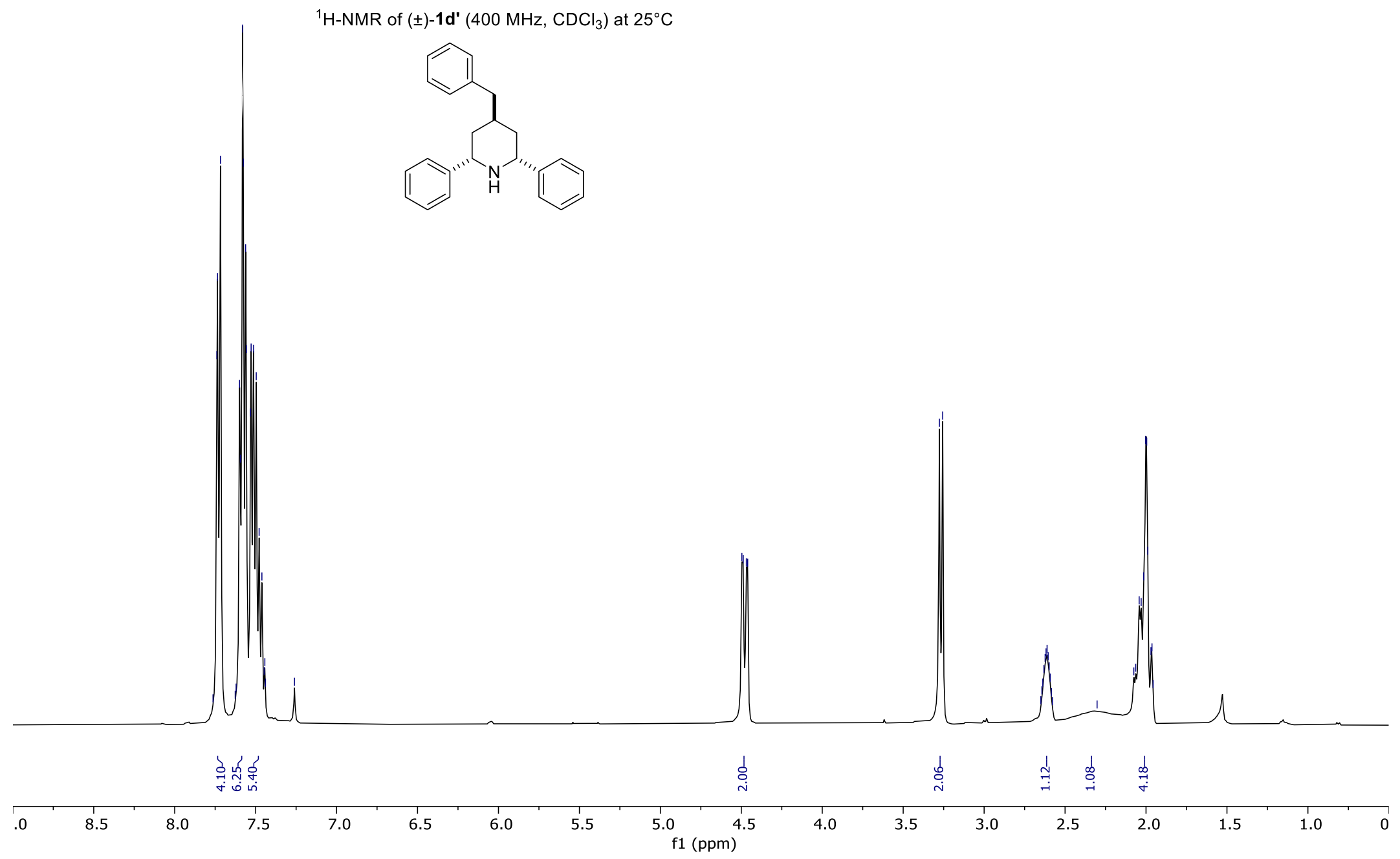

S-81 


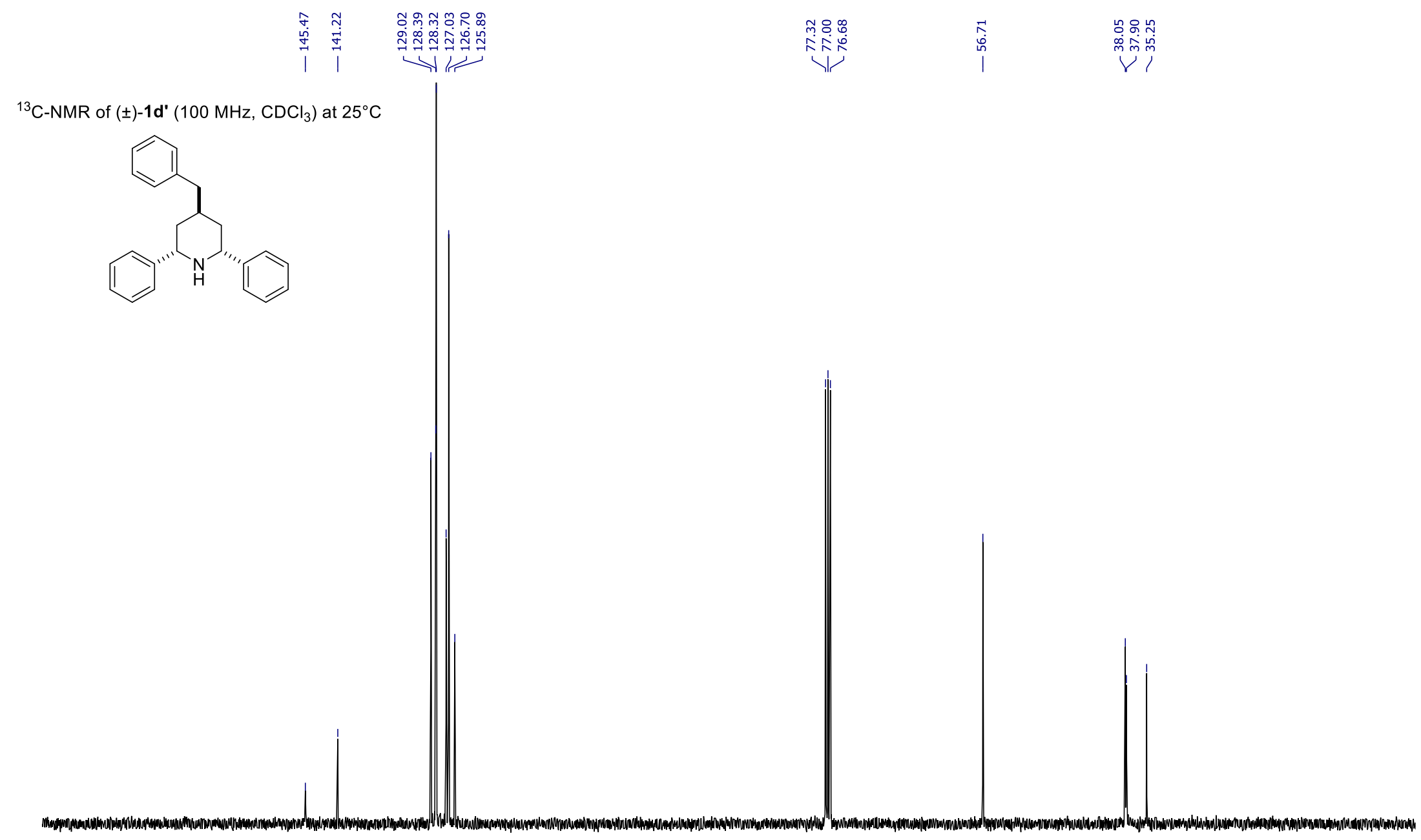

$80+170+160+150$

130

120

110

100 f1 (ppm) 


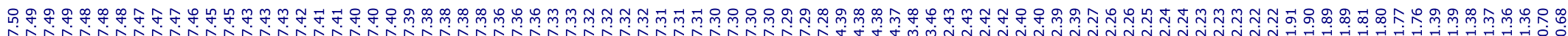

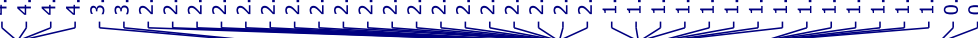

${ }^{1} \mathrm{H}-\mathrm{NMR}$ of $( \pm)-1 \mathrm{e}\left(400 \mathrm{MHz}, \mathrm{CDCl}_{3}\right)$ at $25^{\circ} \mathrm{C}$<smiles>C[C@H]1CC[C@@H](c2ccccc2)NC1c1ccccc1</smiles>

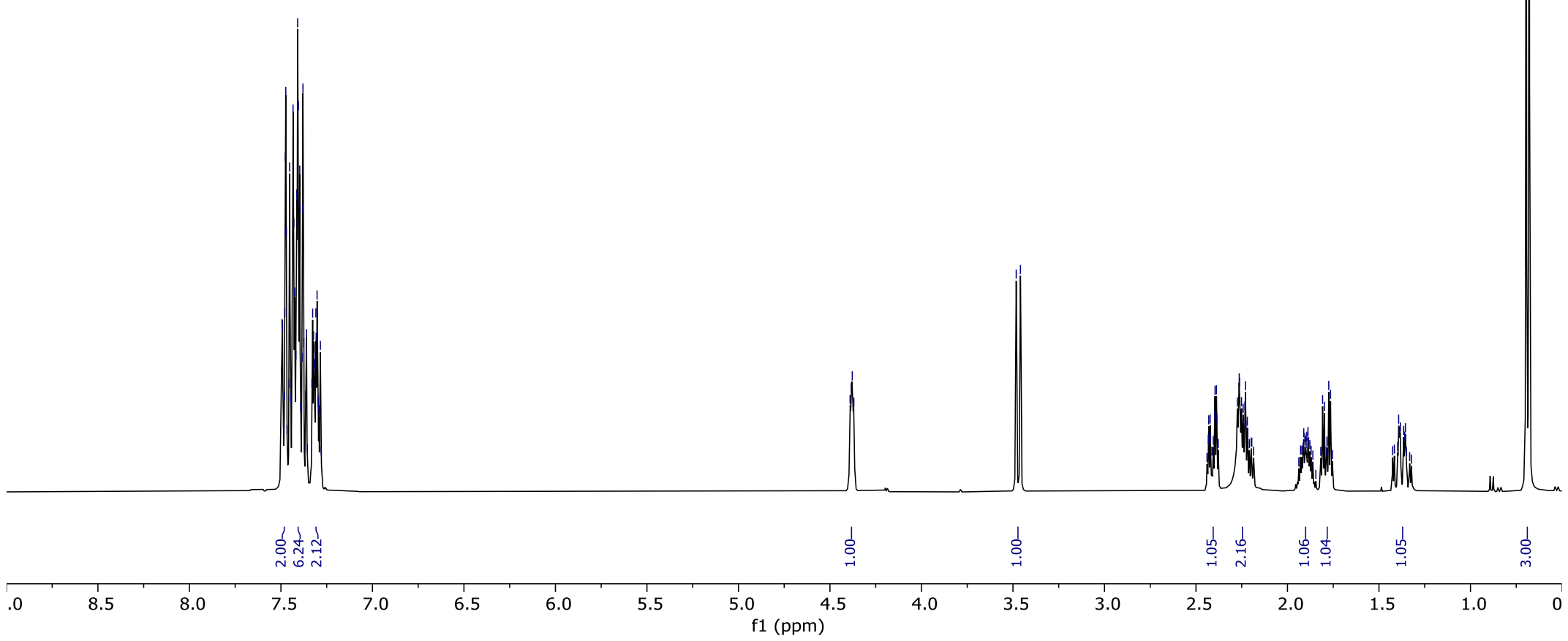



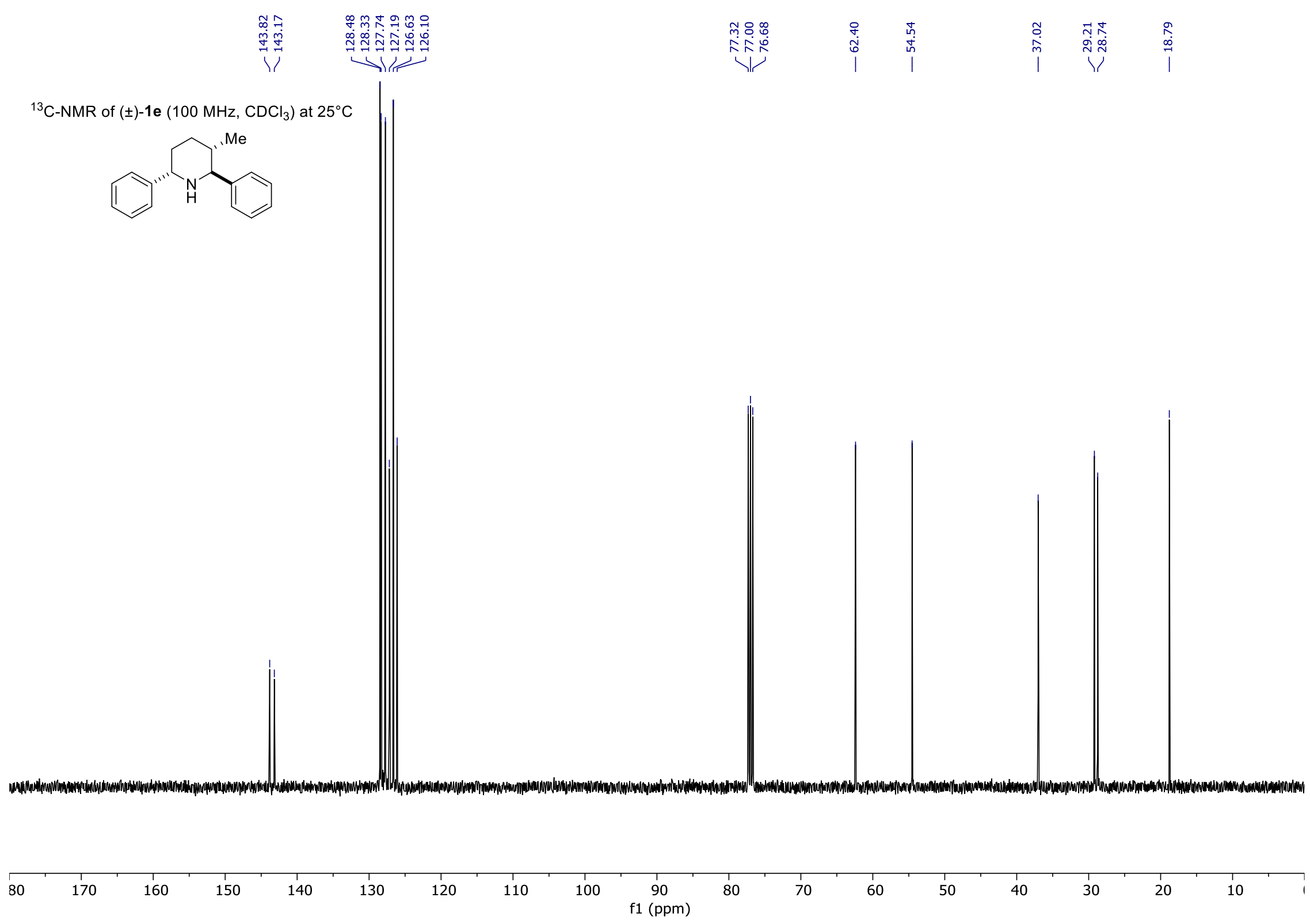

S-84 


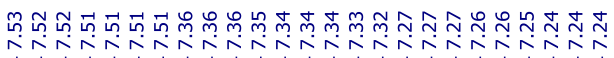

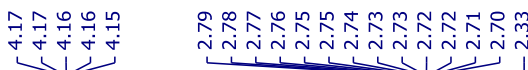

${ }^{1} \mathrm{H}-\mathrm{NMR}$ of $( \pm)-1 \mathrm{f}\left(500 \mathrm{MHz}, \mathrm{CDCl}_{3}\right)$ at $25^{\circ} \mathrm{C}$
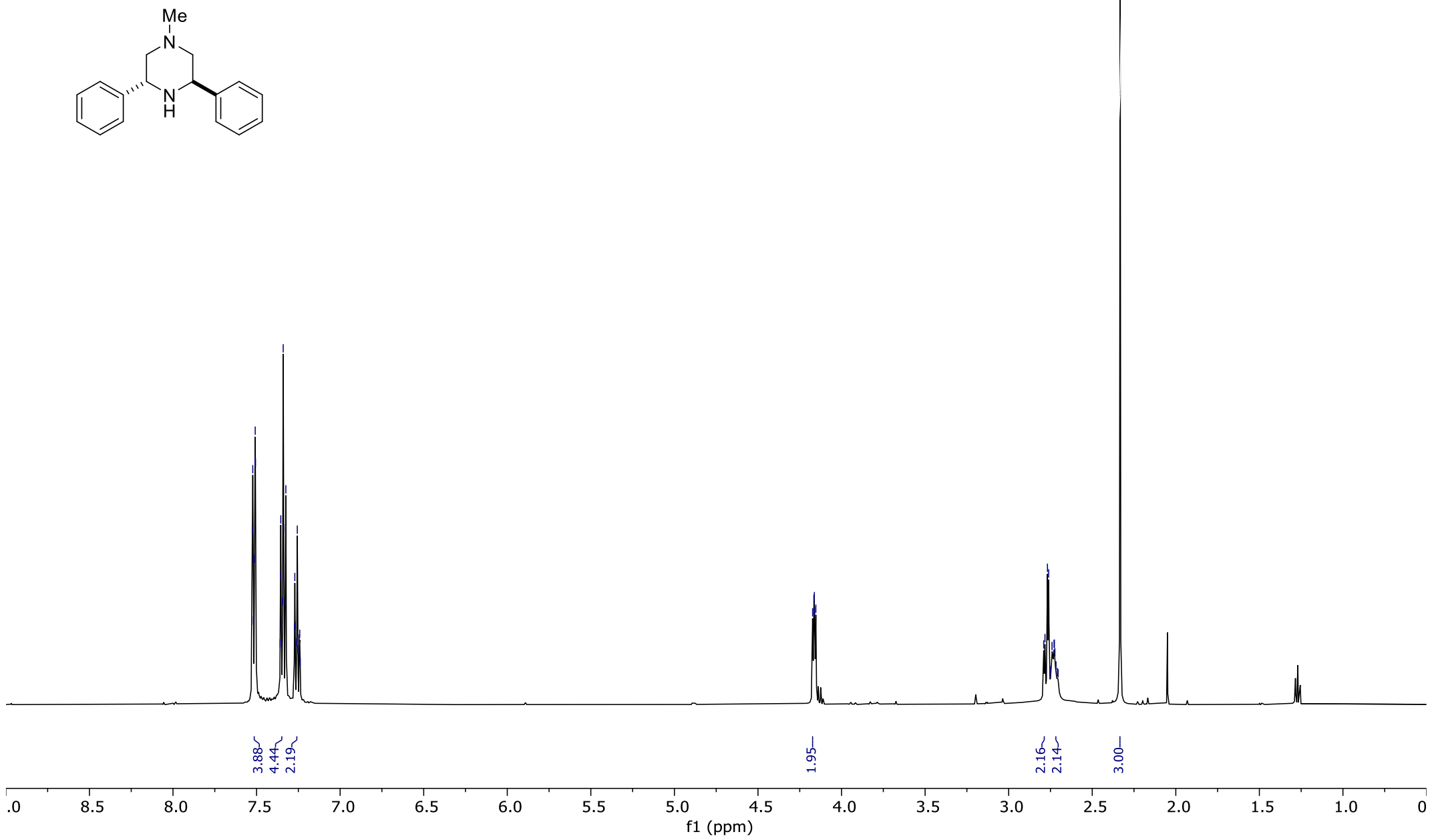

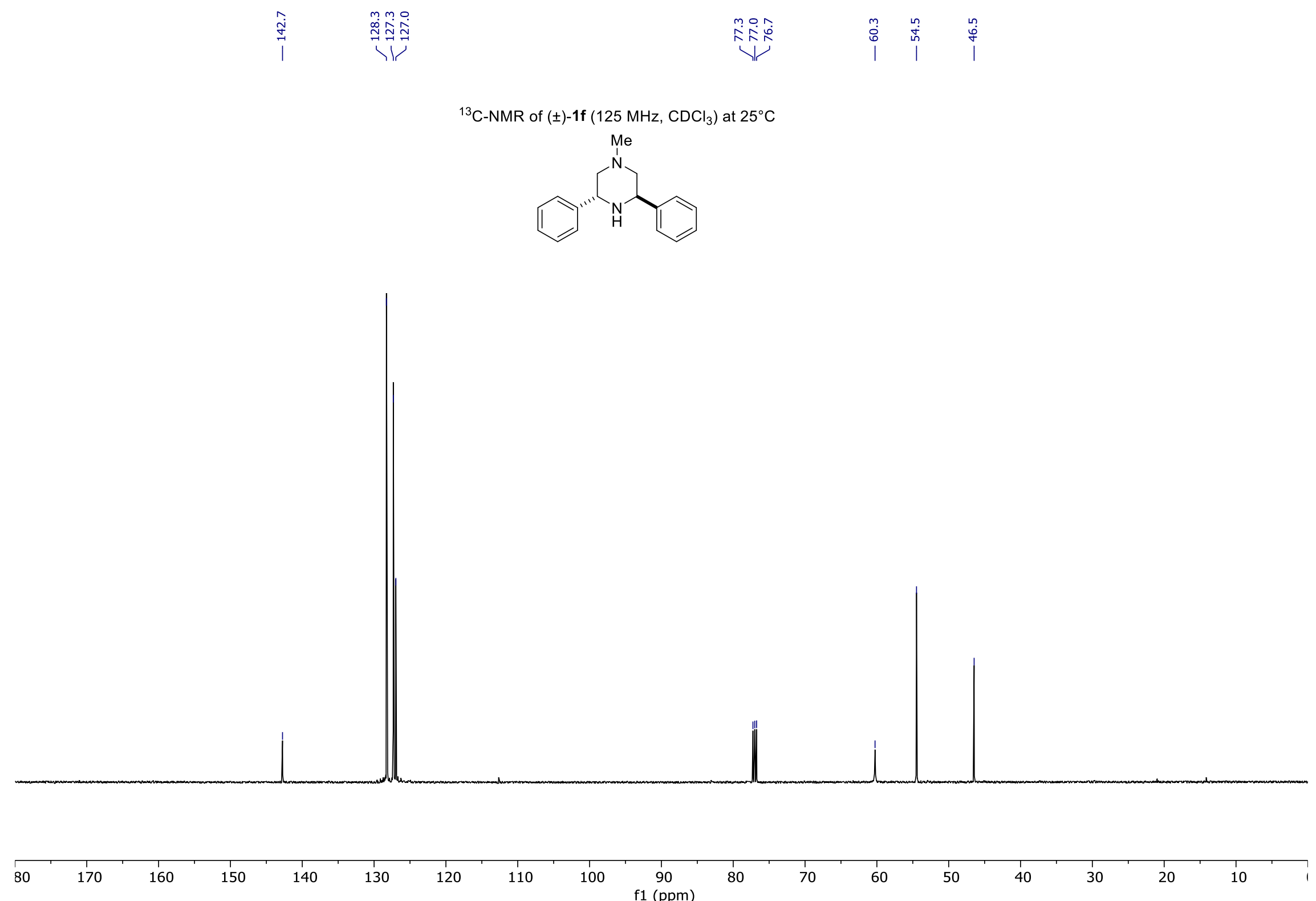


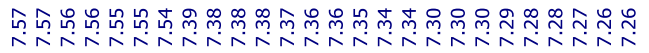

${ }^{1} \mathrm{H}-\mathrm{NMR}$ of $( \pm)-\mathbf{1 g}$ in $\left(400 \mathrm{MHz}, \mathrm{CDCl}_{3}\right)$ at $25^{\circ} \mathrm{C}$

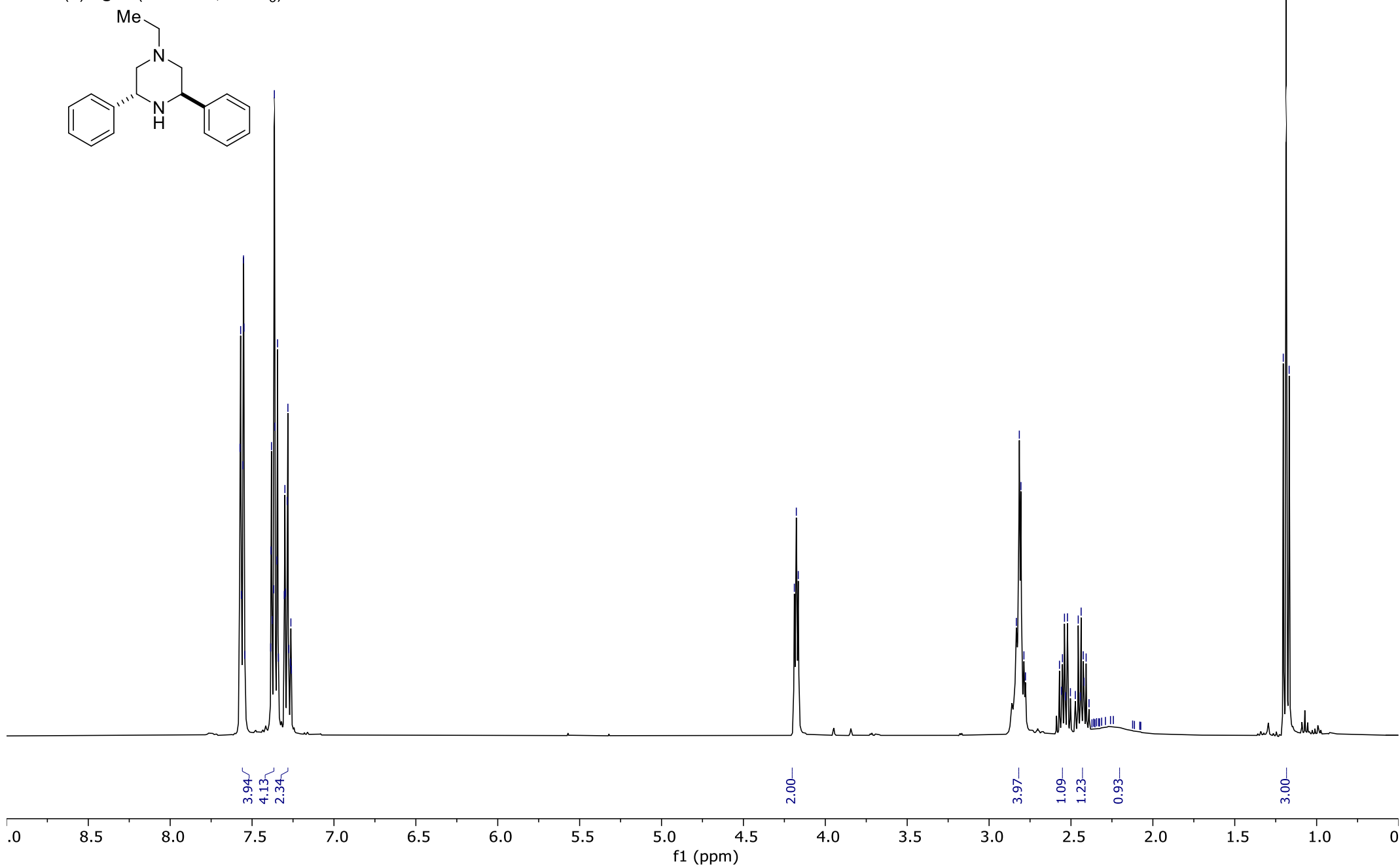


${ }^{13} \mathrm{C}-\mathrm{NMR}$ of $( \pm)-1 \mathrm{~g}\left(100 \mathrm{MHz}, \mathrm{CDCl}_{3}\right)$ at $25^{\circ} \mathrm{C}$
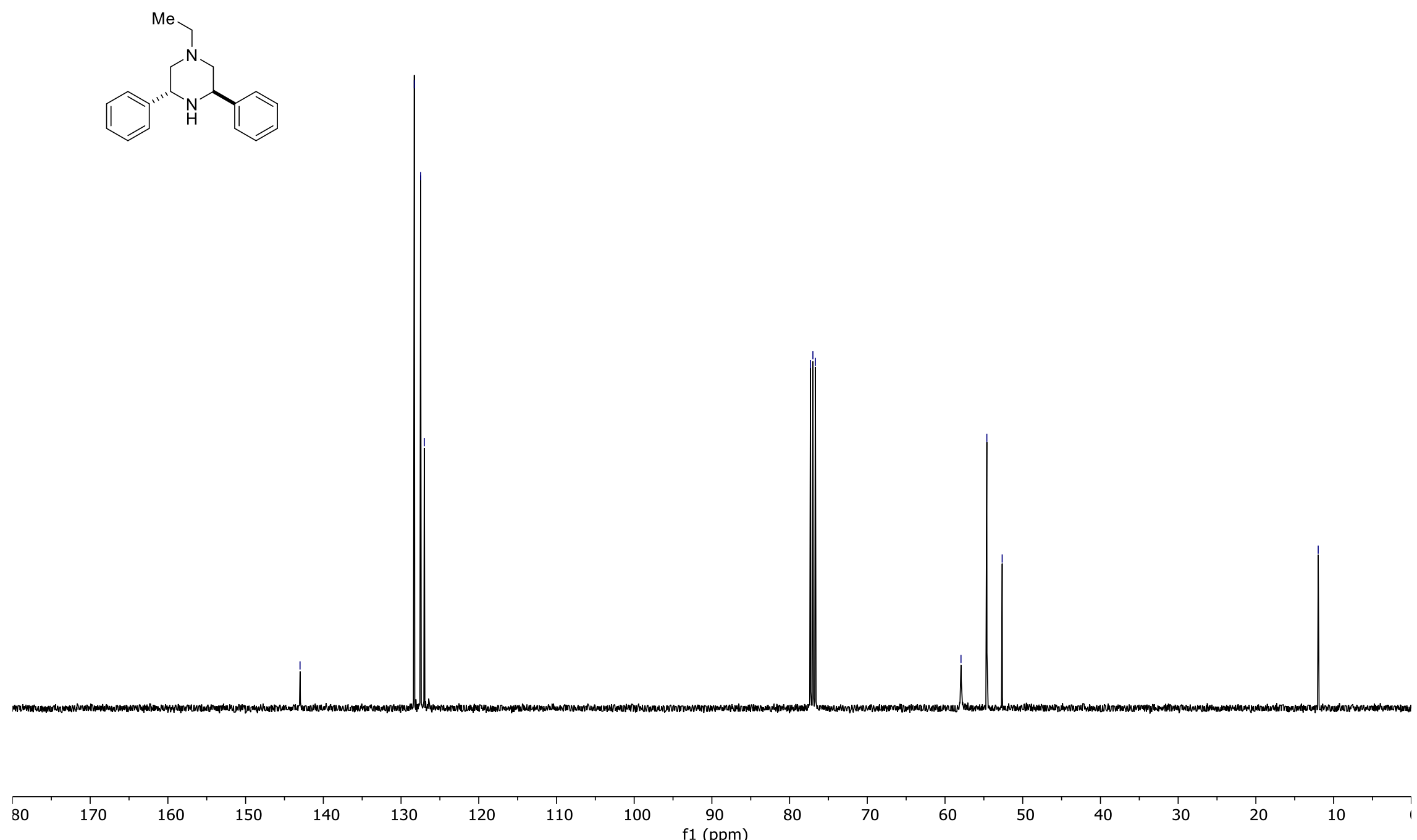


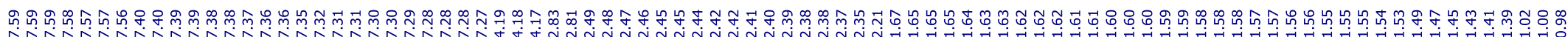

${ }^{1} \mathrm{H}-\mathrm{NMR}$ of $( \pm)-1 \mathrm{~h}\left(400 \mathrm{MHz}, \mathrm{CDCl}_{3}\right)$ at $25^{\circ} \mathrm{C}$

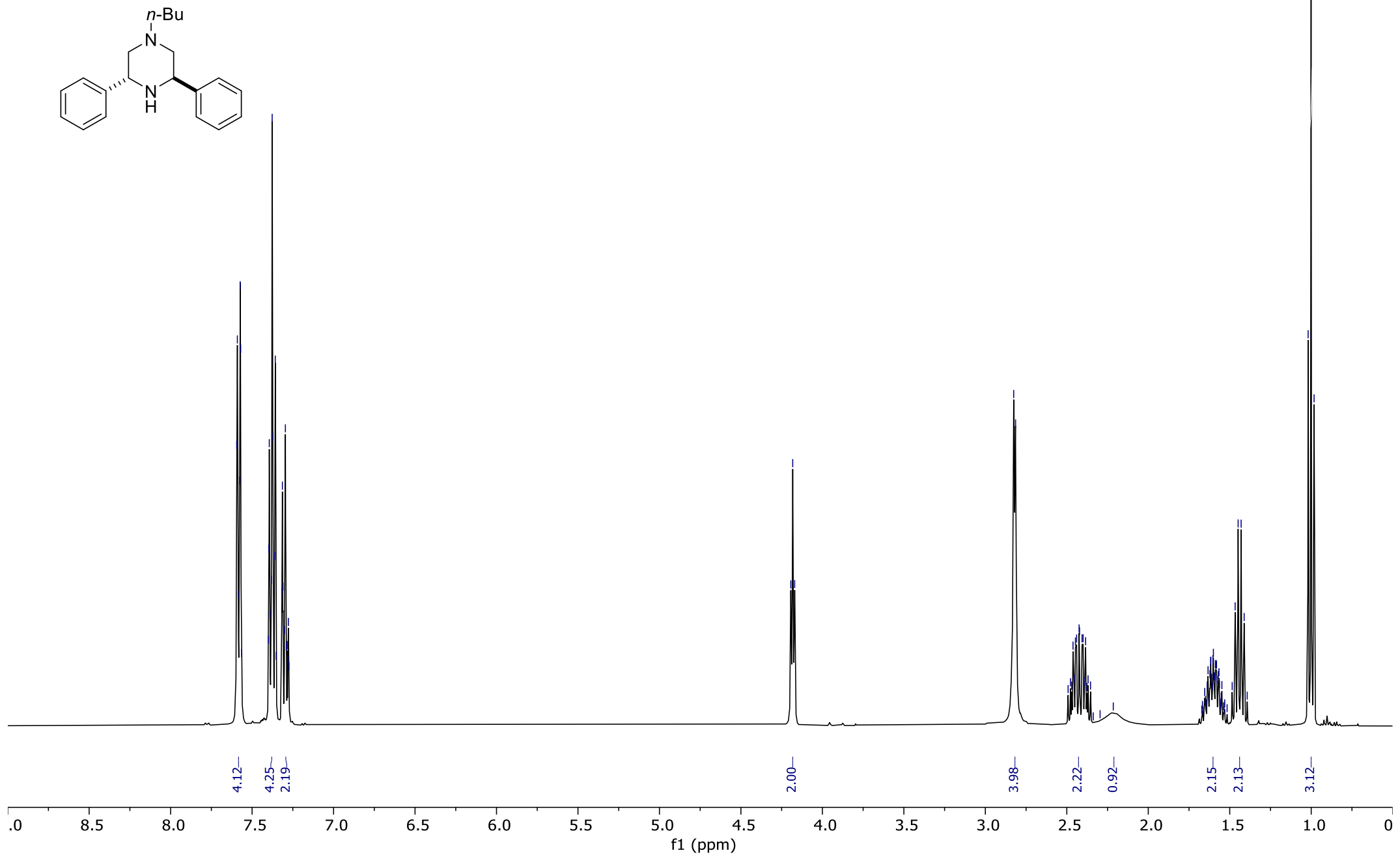




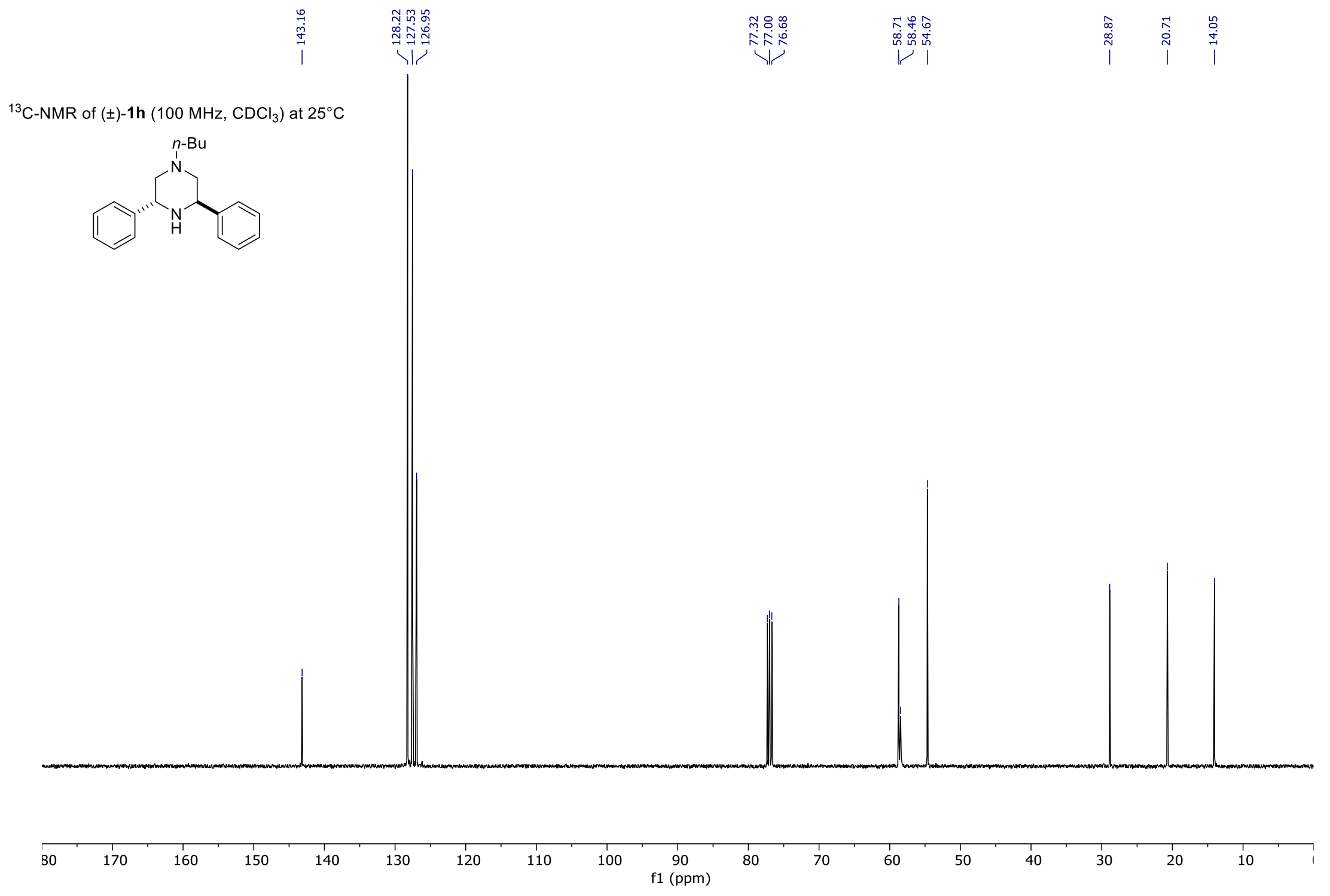


${ }^{1} \mathrm{H}-\mathrm{NMR}$ of $( \pm)-1 \mathrm{i}\left(300 \mathrm{MHz}, \mathrm{CDCl}_{3}\right)$ at $25^{\circ} \mathrm{C}$

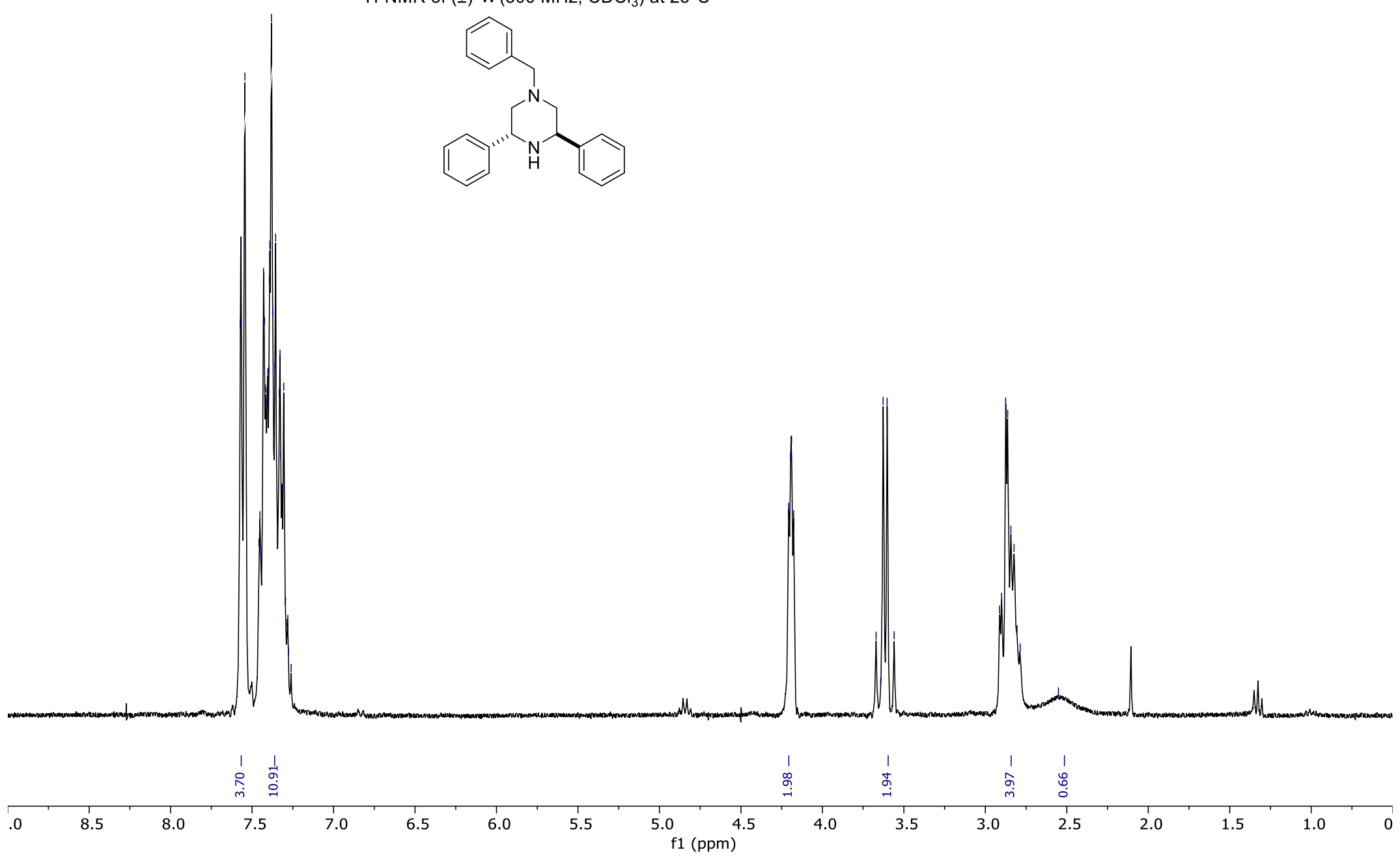




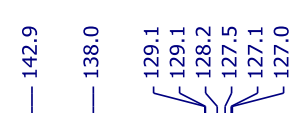$$
\text { 经 }
$$

${ }^{13} \mathrm{C}-\mathrm{NMR}$ of $( \pm)-1 \mathbf{i}\left(75 \mathrm{MHz}, \mathrm{CDCl}_{3}\right)$ at $25^{\circ} \mathrm{C}$
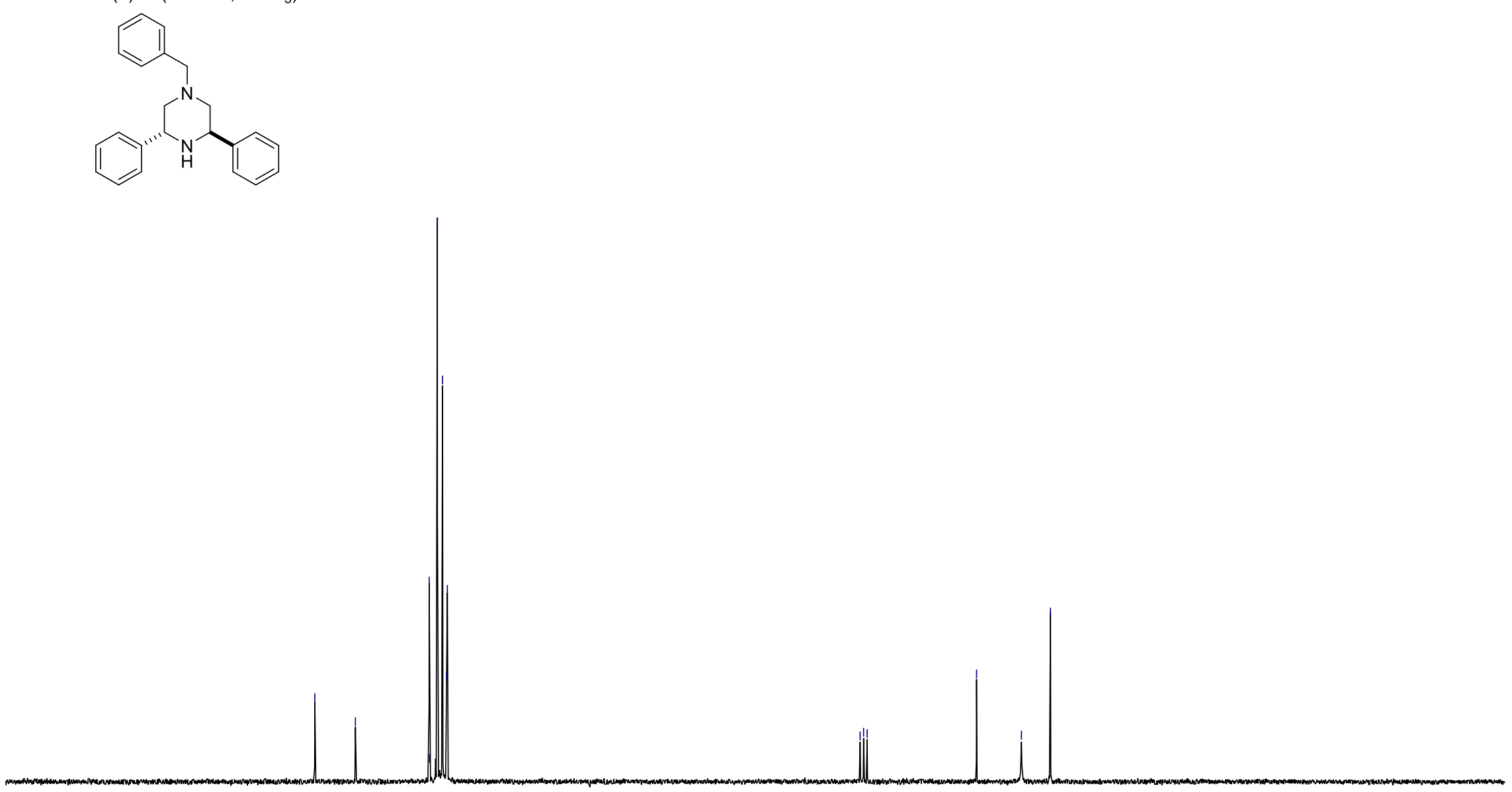

80

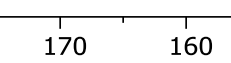

150
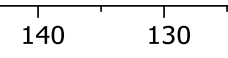

120 


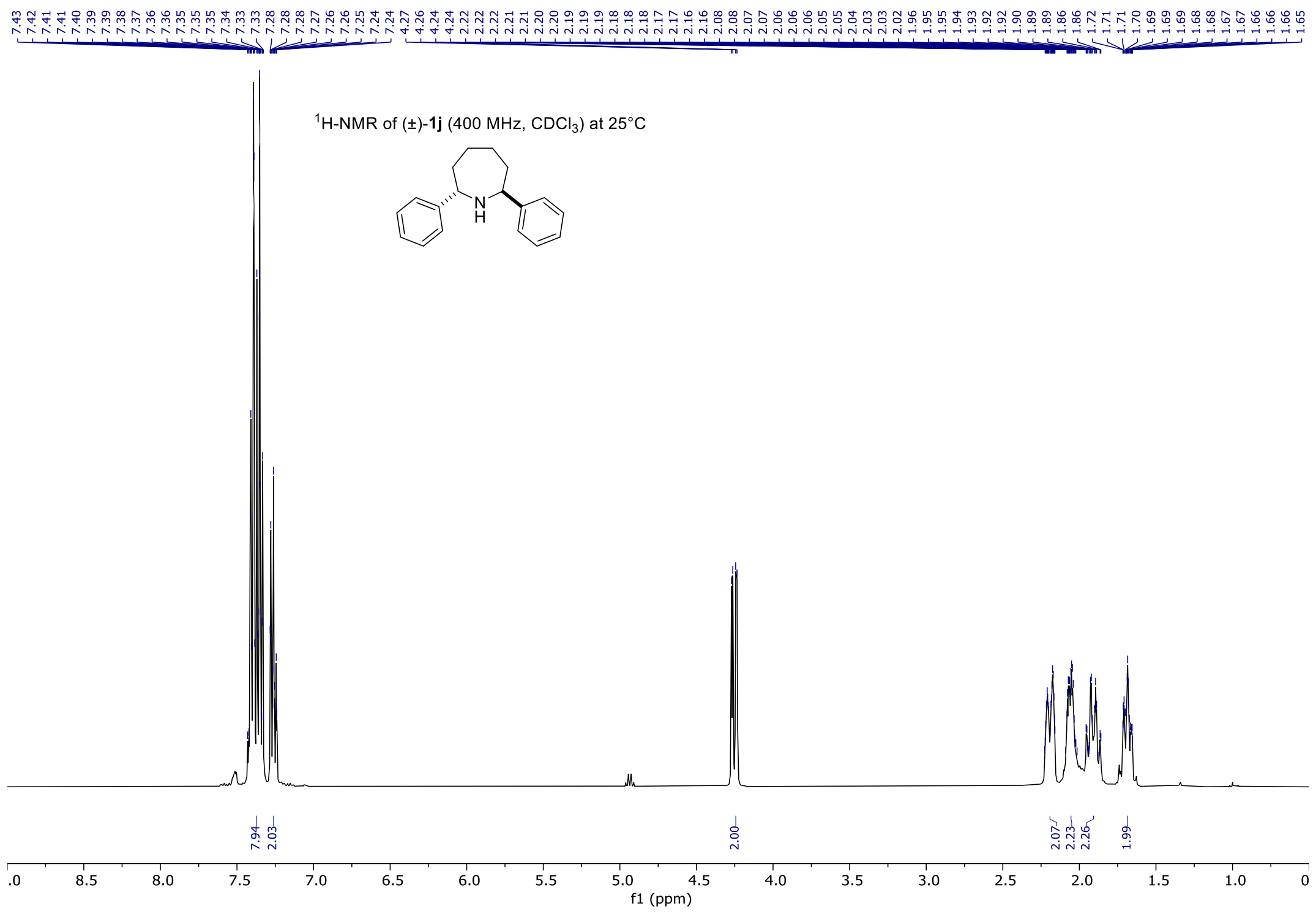



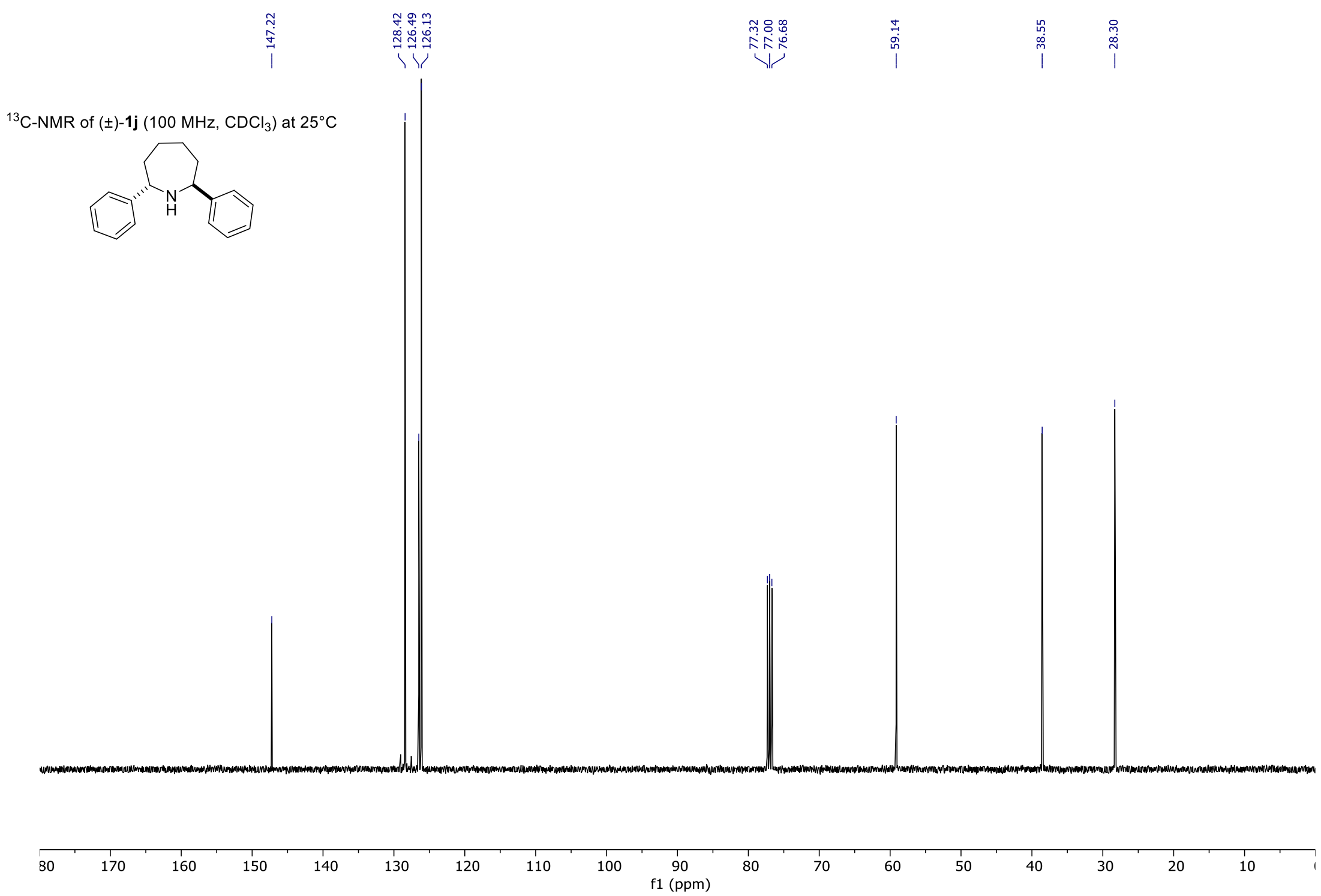


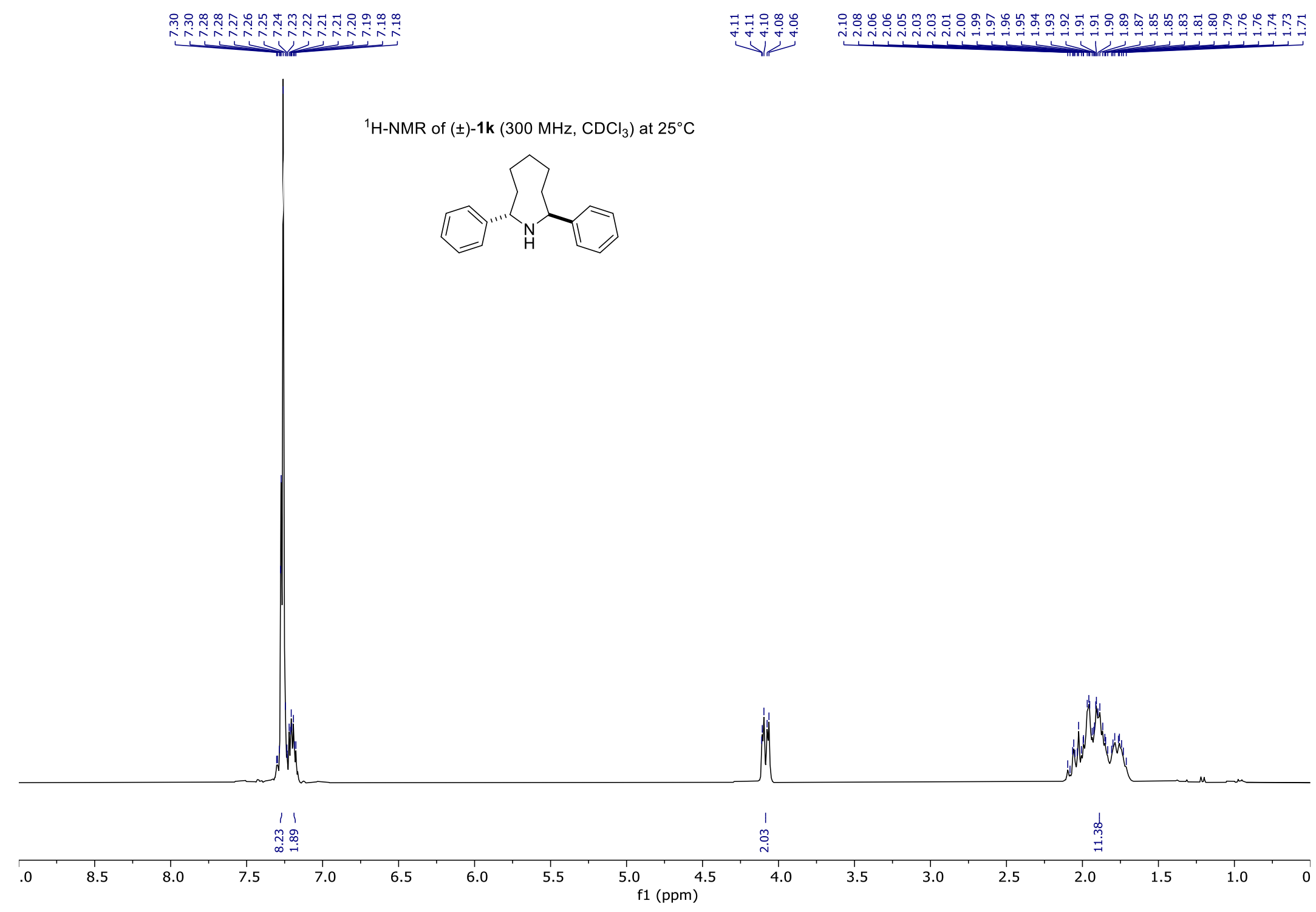




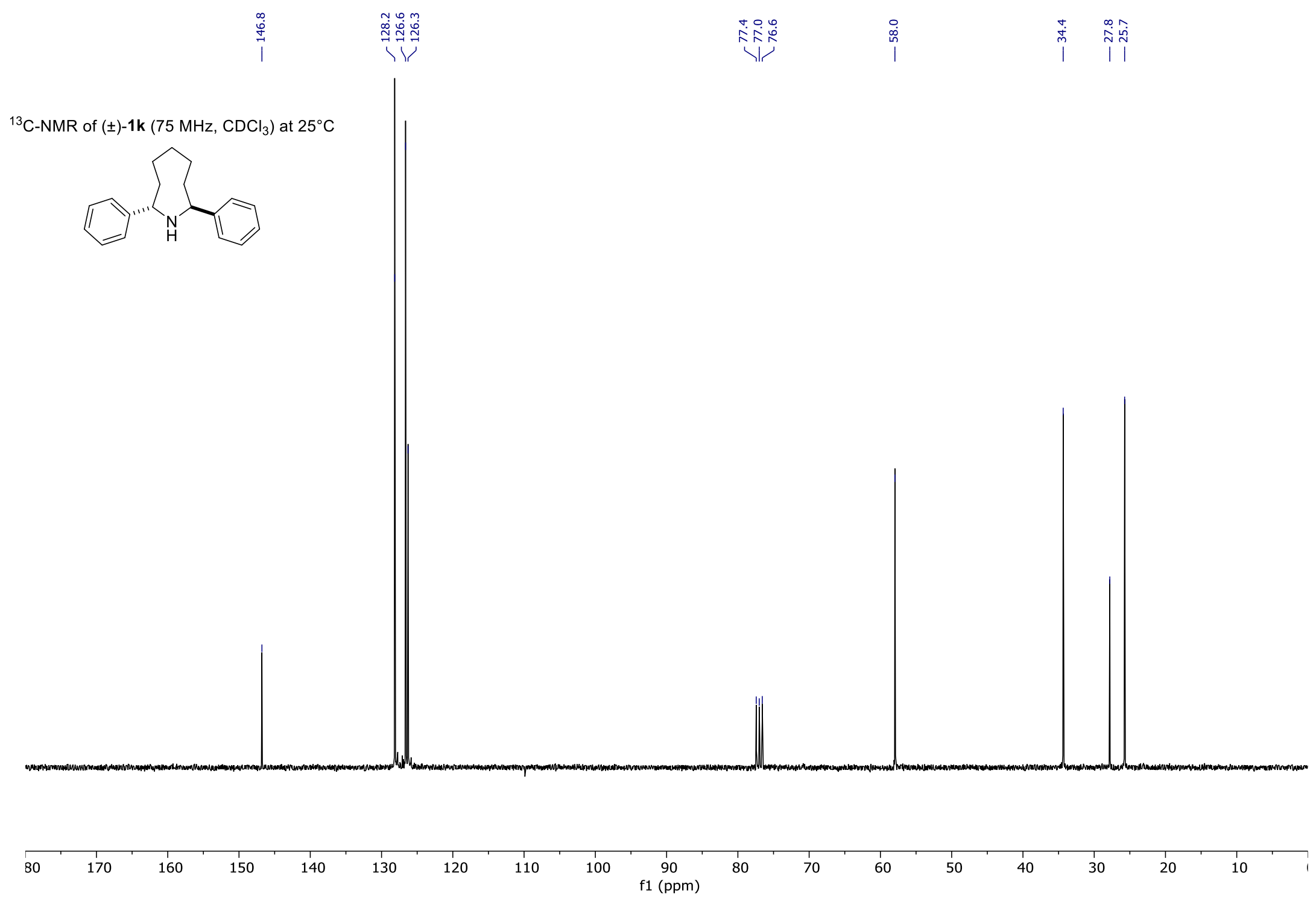




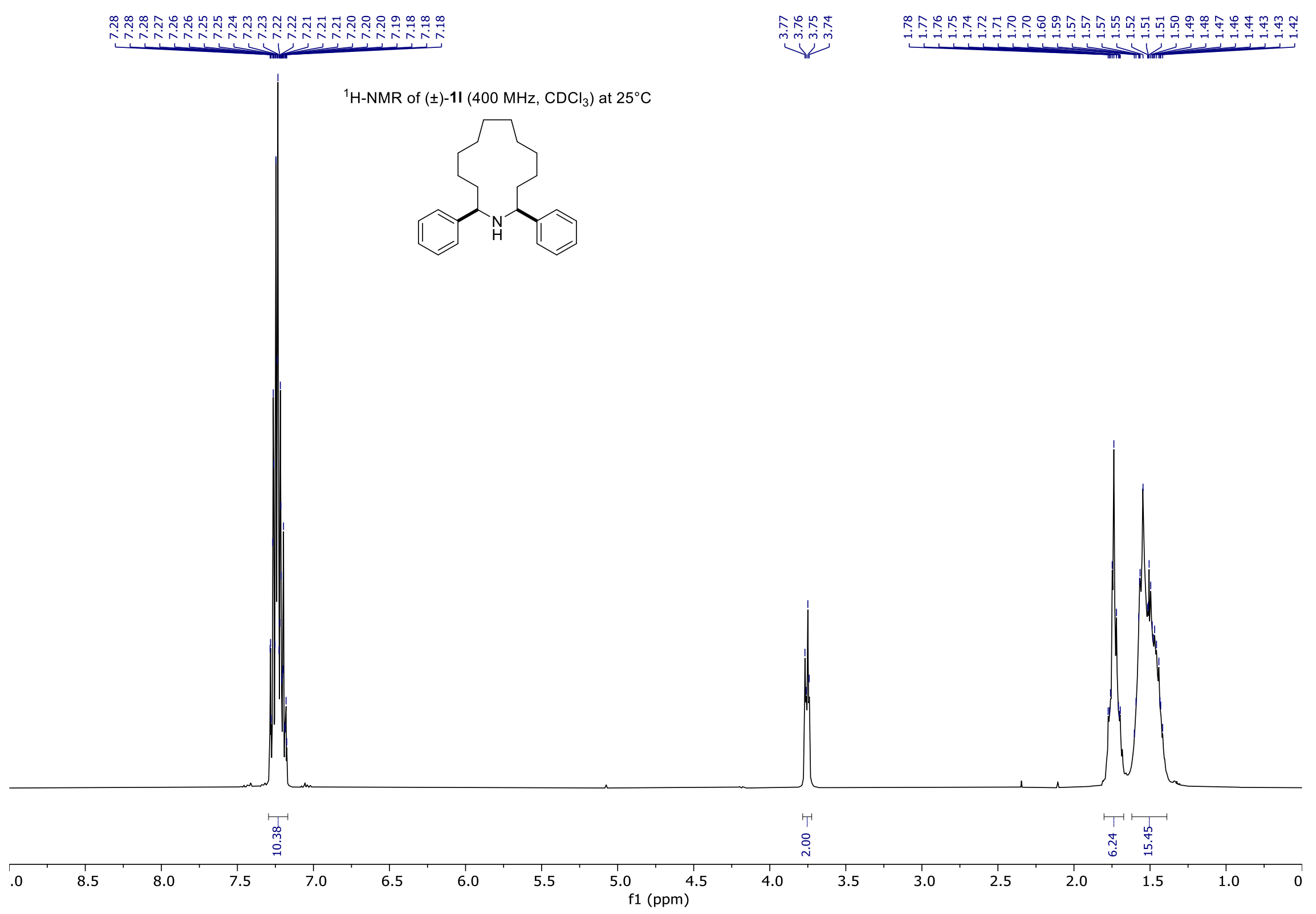

S-97 


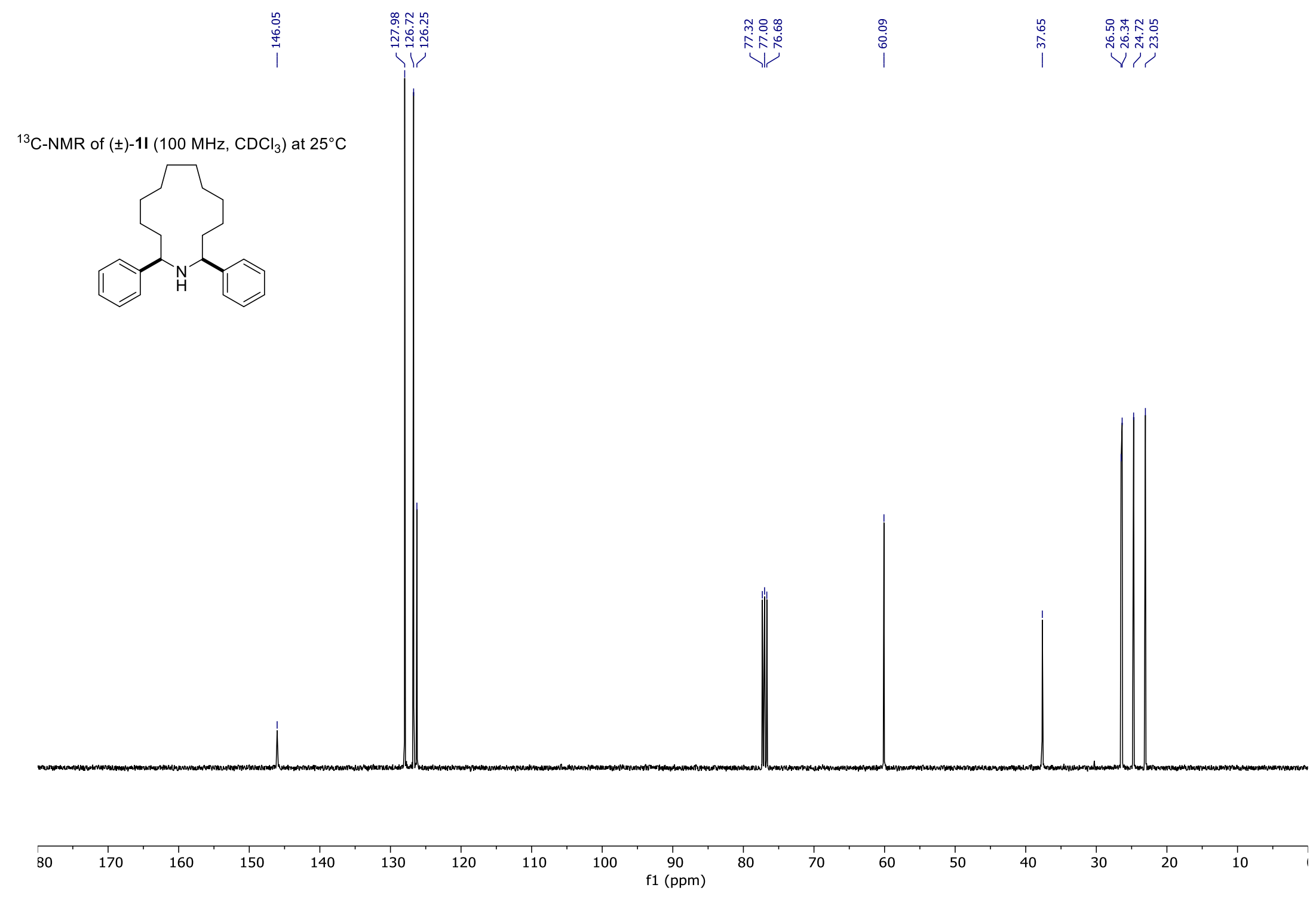




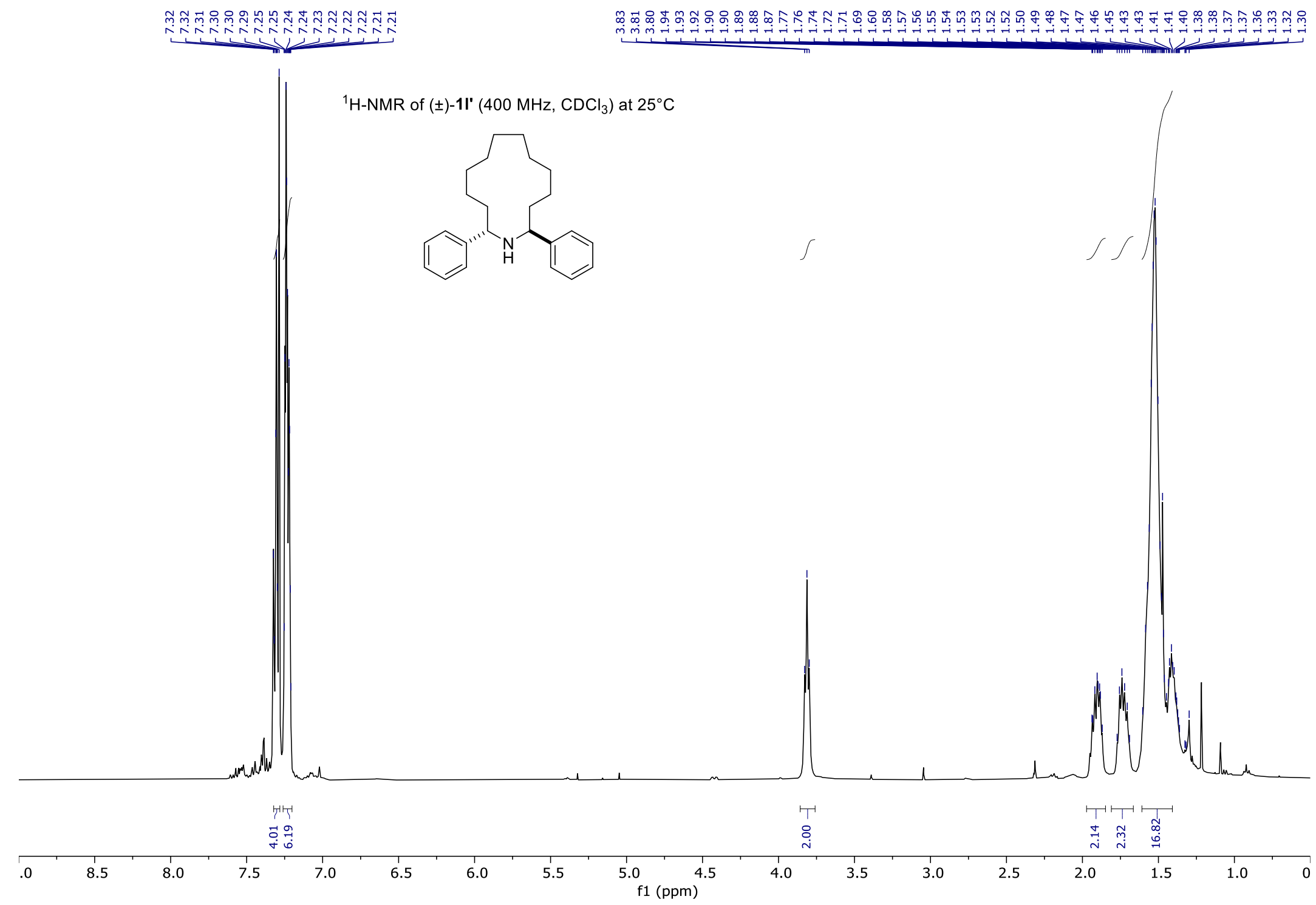



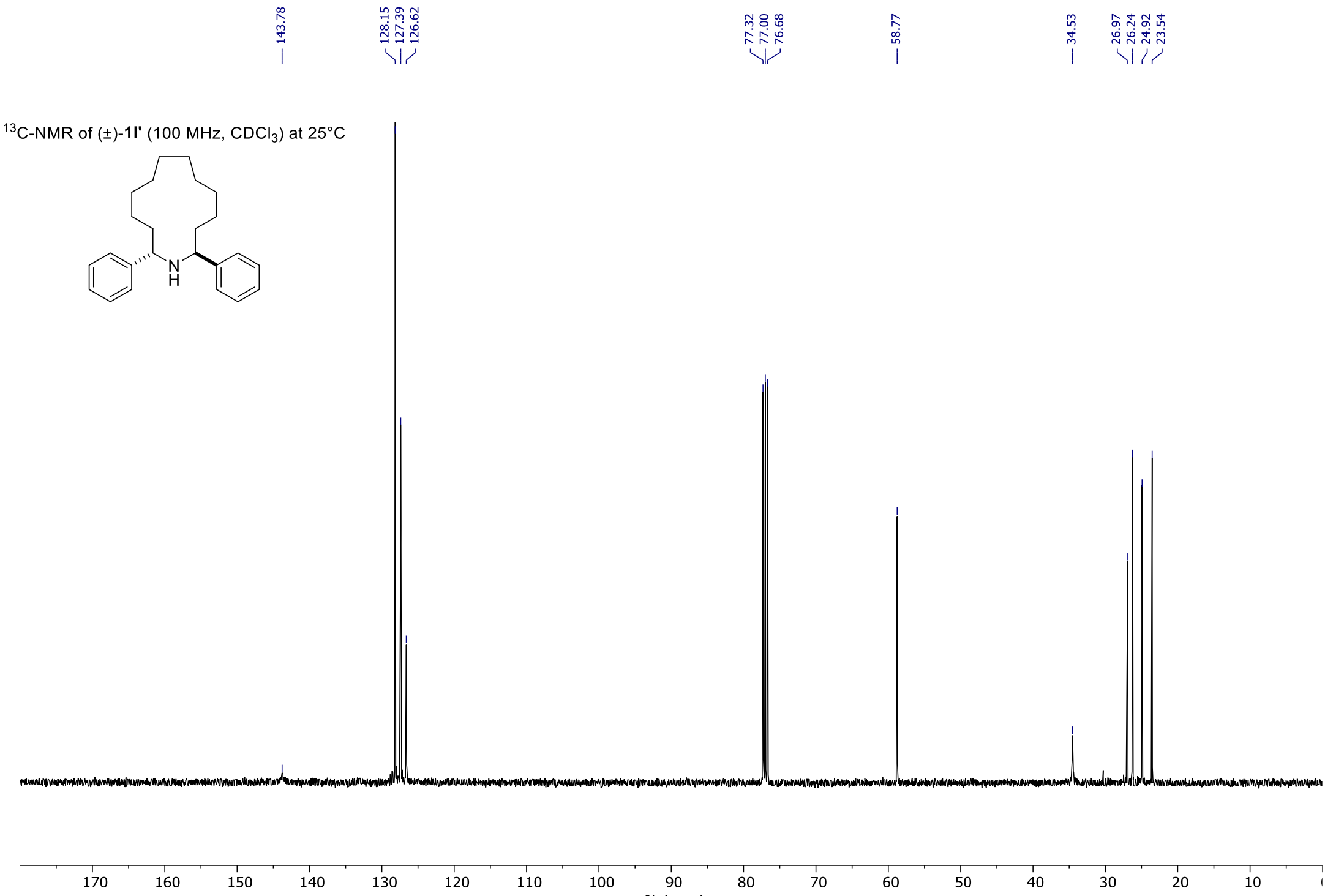

120

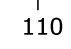

100

90
f1 $(p p m)$

80

70

60

50

40

30

20 


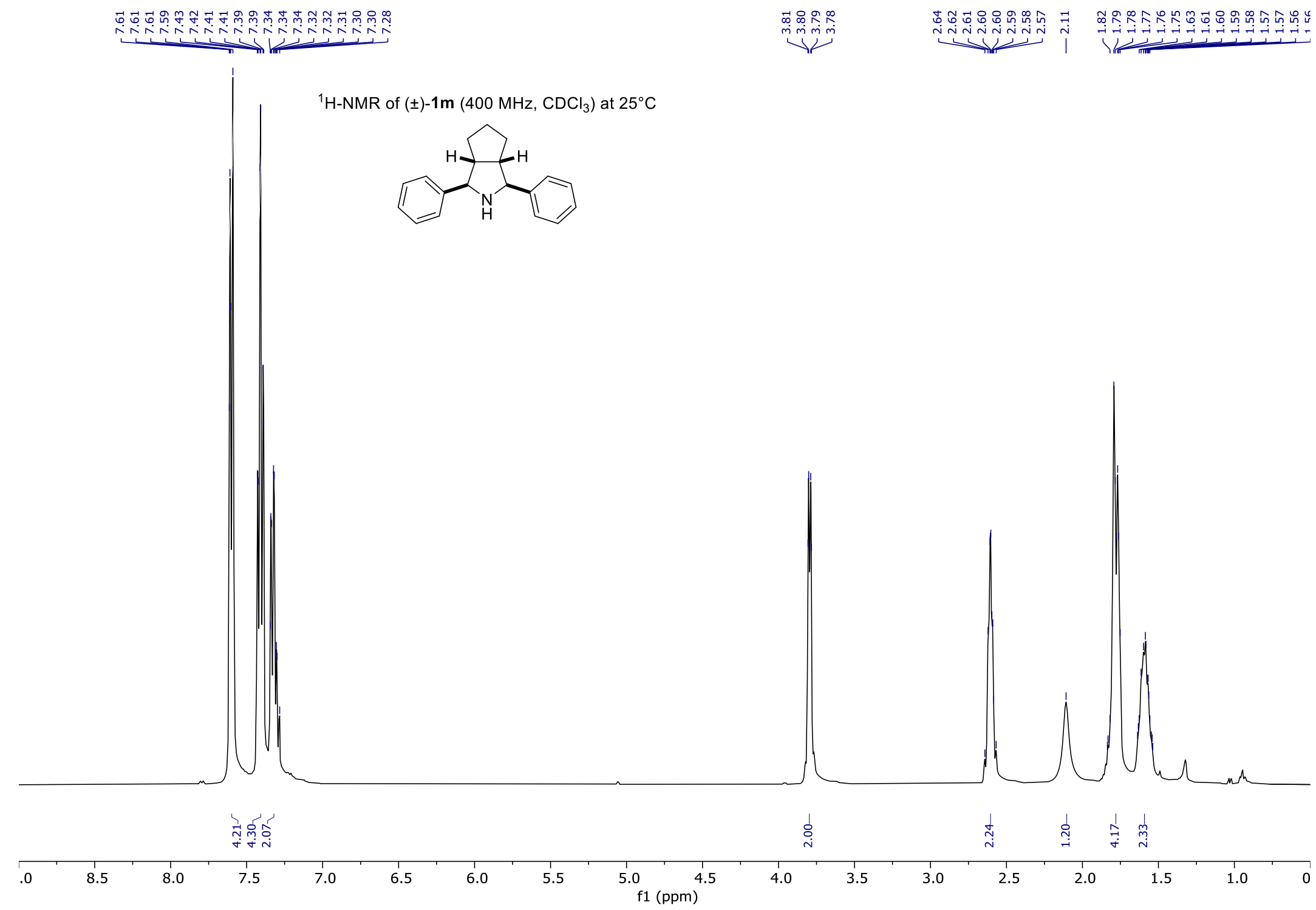

S-101 
${ }^{13} \mathrm{C}-\mathrm{NMR}$ of $( \pm)-1 \mathrm{~m}\left(100 \mathrm{MHz}, \mathrm{CDCl}_{3}\right)$ at $25^{\circ} \mathrm{C}$

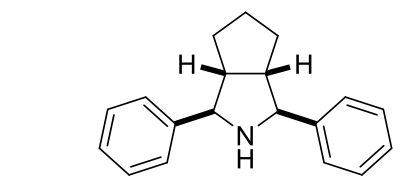

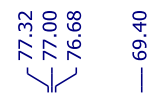

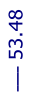

$\begin{array}{ll}\stackrel{0}{1} & \infty \\ 0 & \stackrel{0}{\sim} \\ 1 & 1\end{array}$

V)
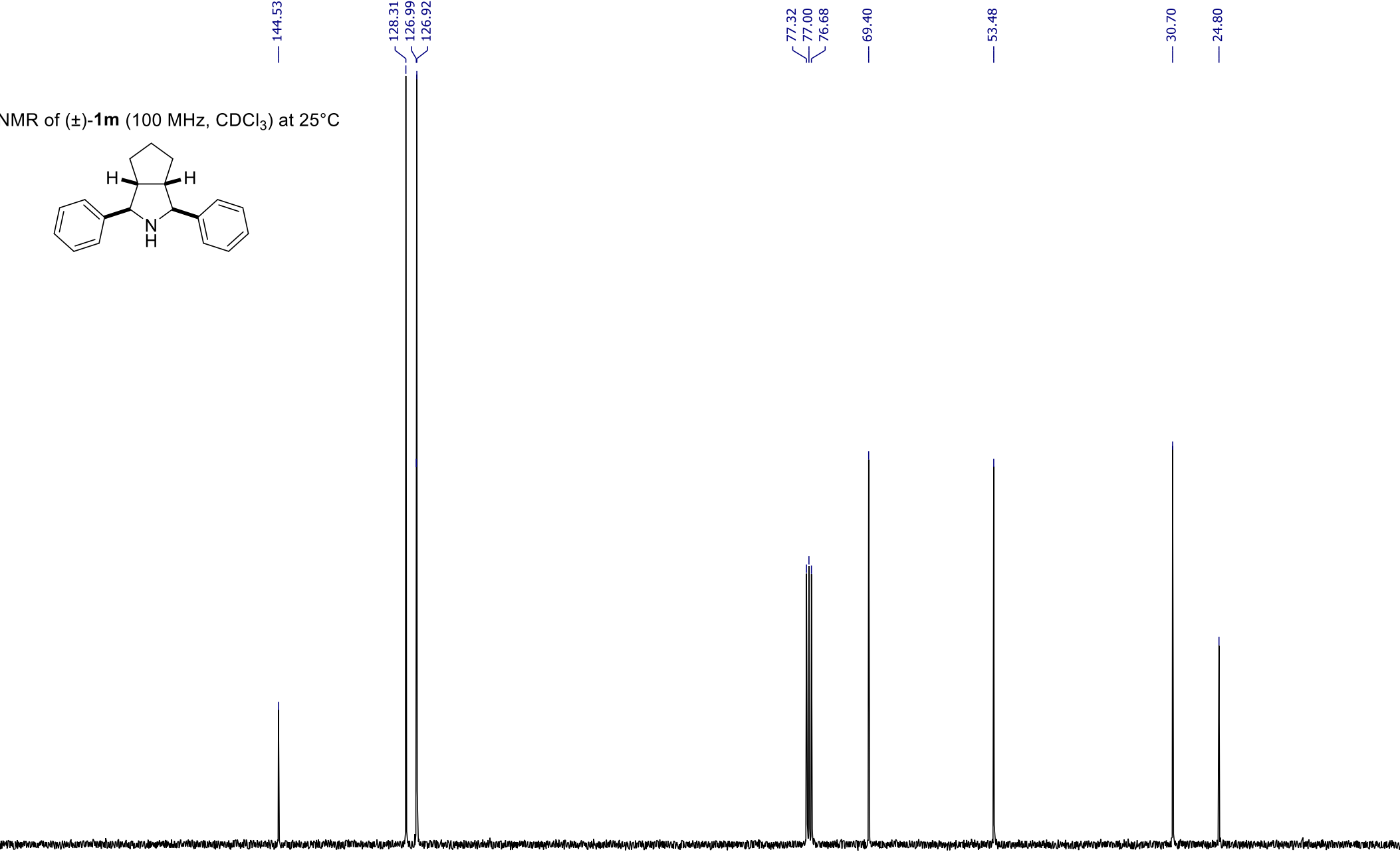

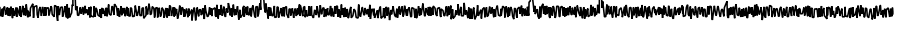

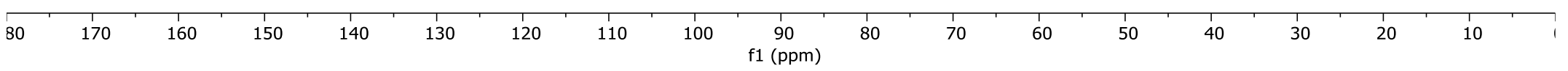

S-102 


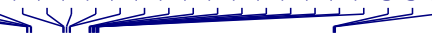

${ }^{1} \mathrm{H}-\mathrm{NMR}$ of $( \pm)-1 \mathrm{n}\left(600 \mathrm{MHz}, \mathrm{CDCl}_{3}\right)$ at $25^{\circ} \mathrm{C}$

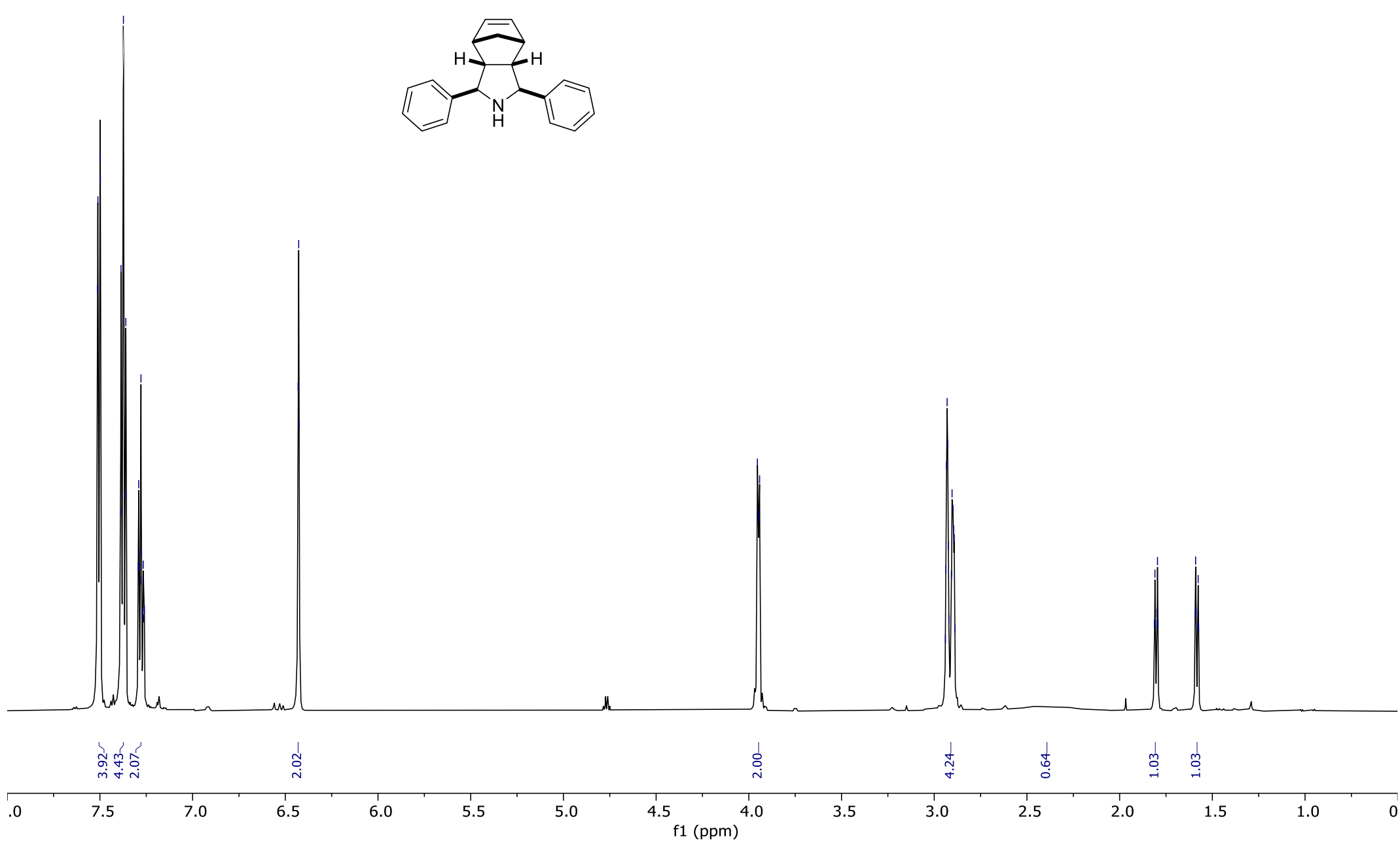


${ }^{13} \mathrm{C}-\mathrm{NMR}$ of $( \pm)-\mathbf{n}\left(151 \mathrm{MHz}, \mathrm{CDCl}_{3}\right)$ at $25^{\circ} \mathrm{C}$
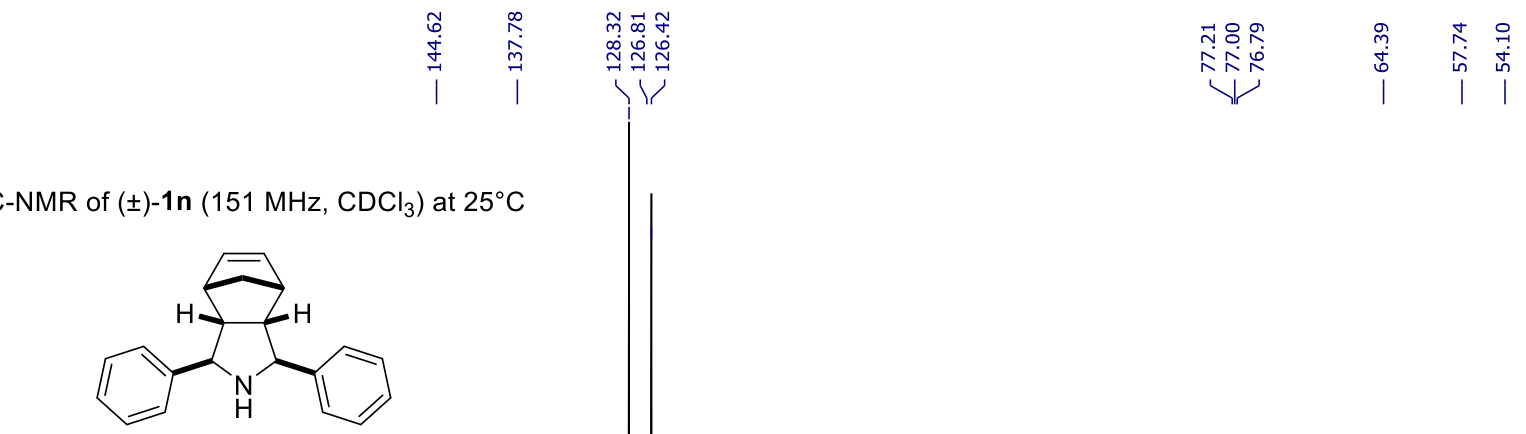

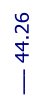
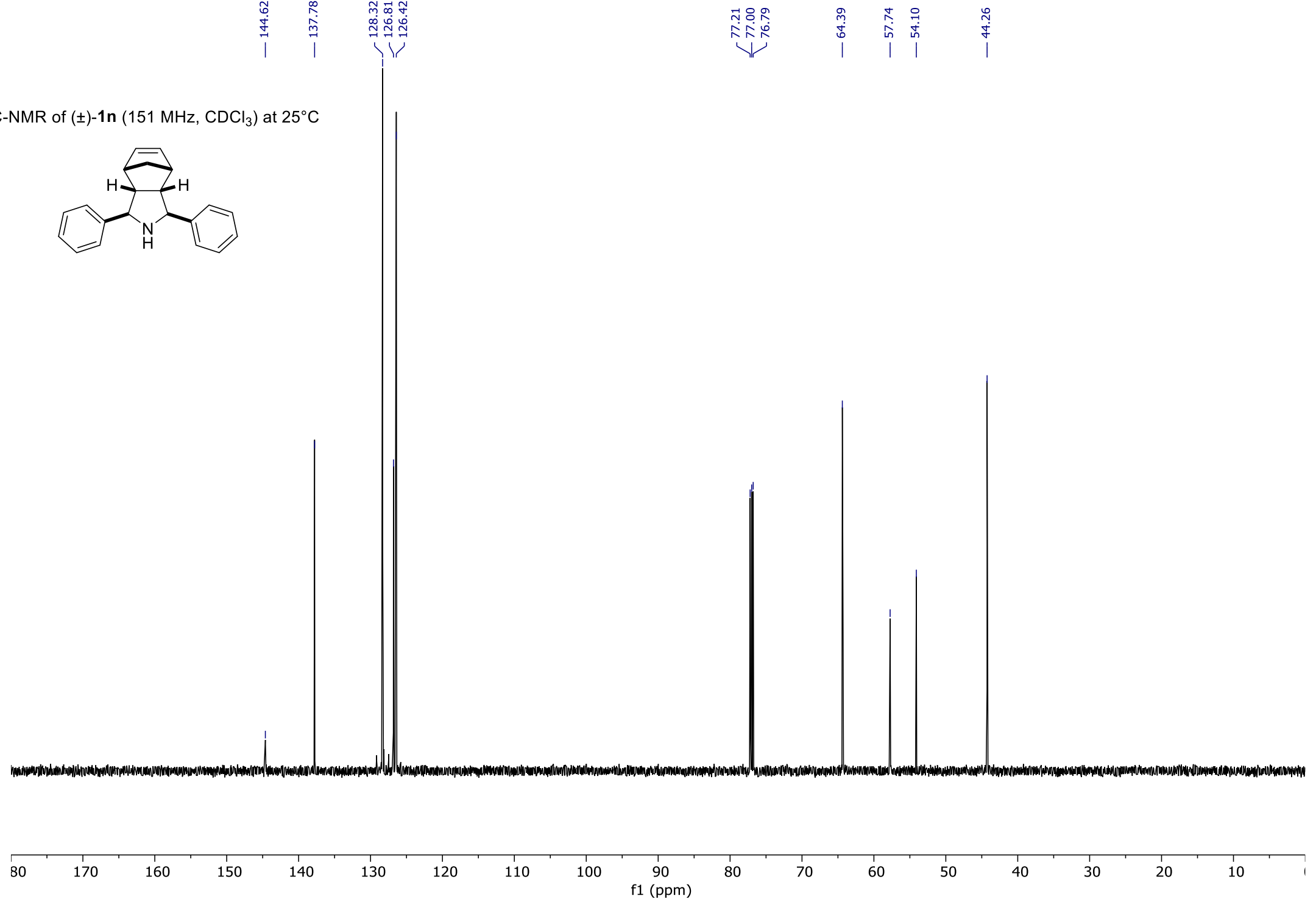

S-104 


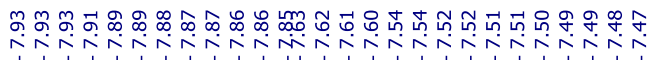

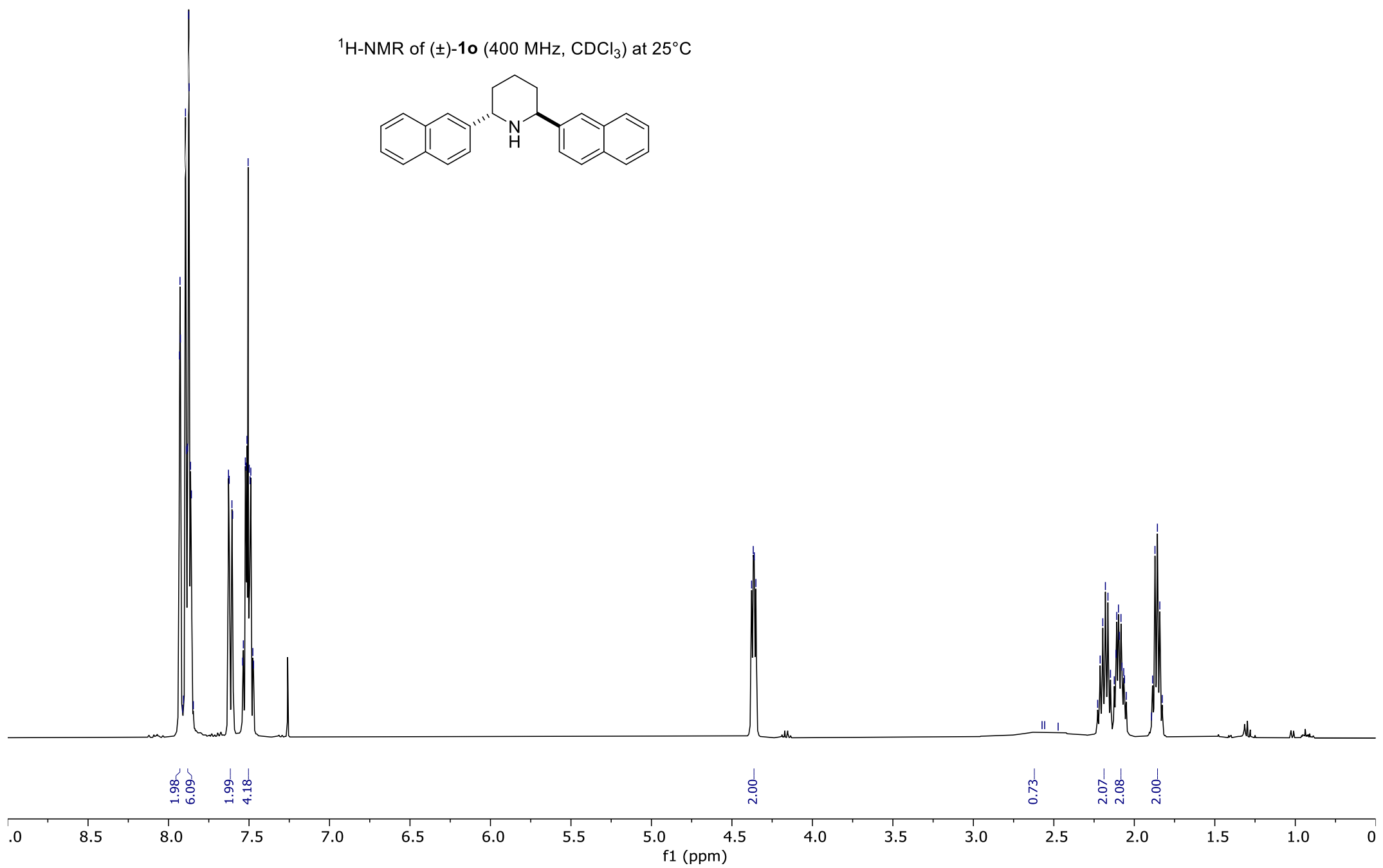

S-105 

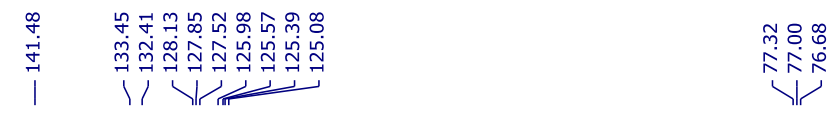

$\stackrel{\substack{m \\ m}}{\stackrel{\infty}{\sim}}$

${ }^{13} \mathrm{C}$-NMR of $( \pm)-10\left(100 \mathrm{MHz}, \mathrm{CDCl}_{3}\right)$ at $25^{\circ} \mathrm{C}$

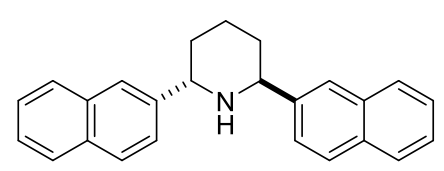

Nown

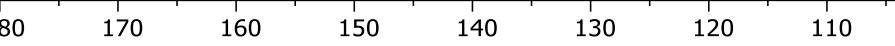

90
f1 $(p p m)$

80

70

50

40

30

20 


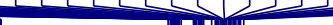

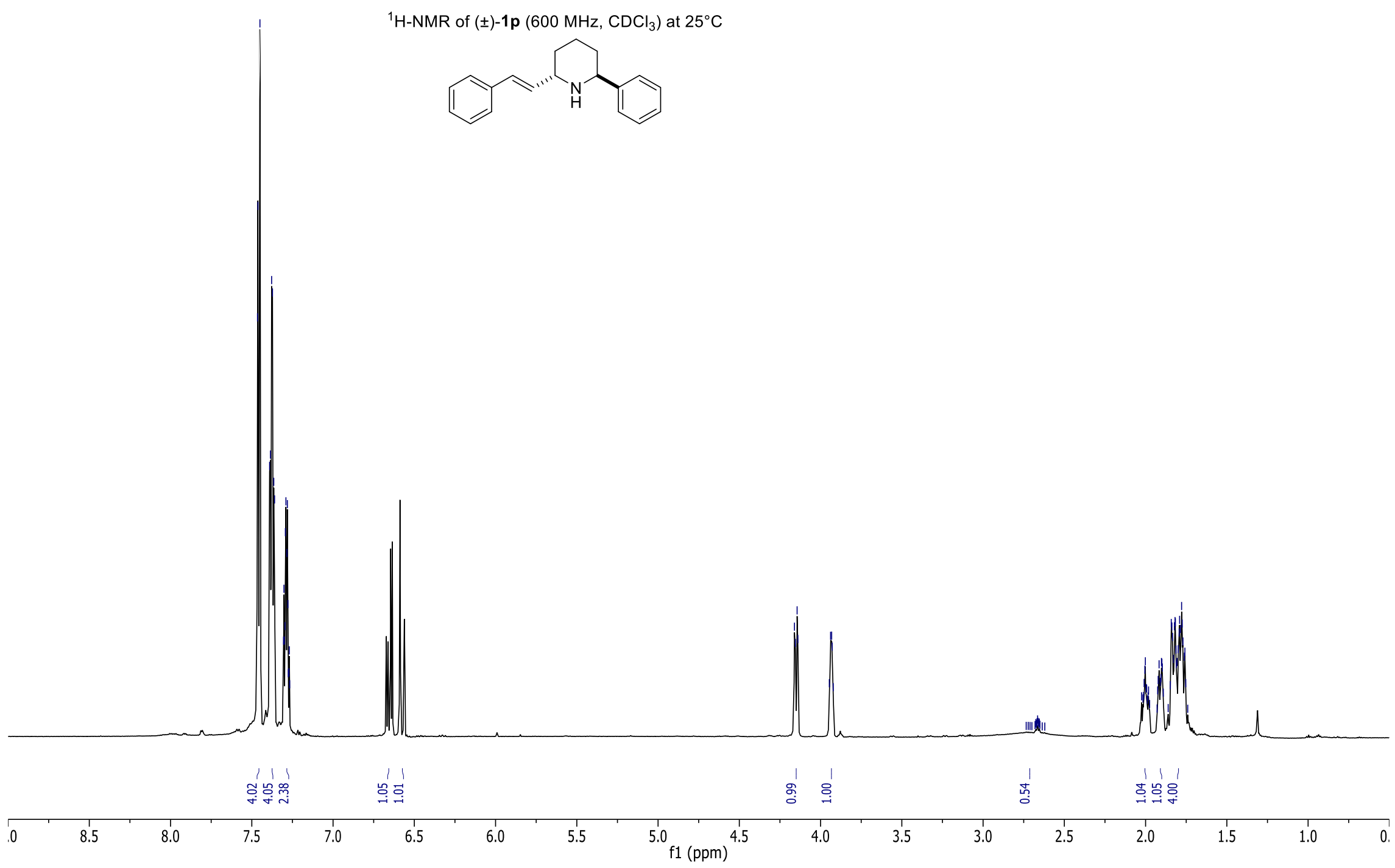

S-107 


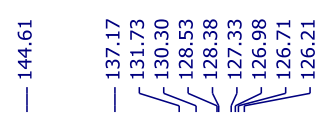

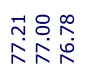

ㅊํㄴ

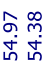

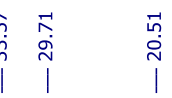

${ }^{13} \mathrm{C}-\mathrm{NMR}$ of $( \pm)-1 \mathrm{p}\left(151 \mathrm{MHz}, \mathrm{CDCl}_{3}\right)$ at $25^{\circ} \mathrm{C}$<smiles>C(=C/[C@H]1CCC[C@@H](c2ccccc2)N1)\c1ccccc1</smiles>
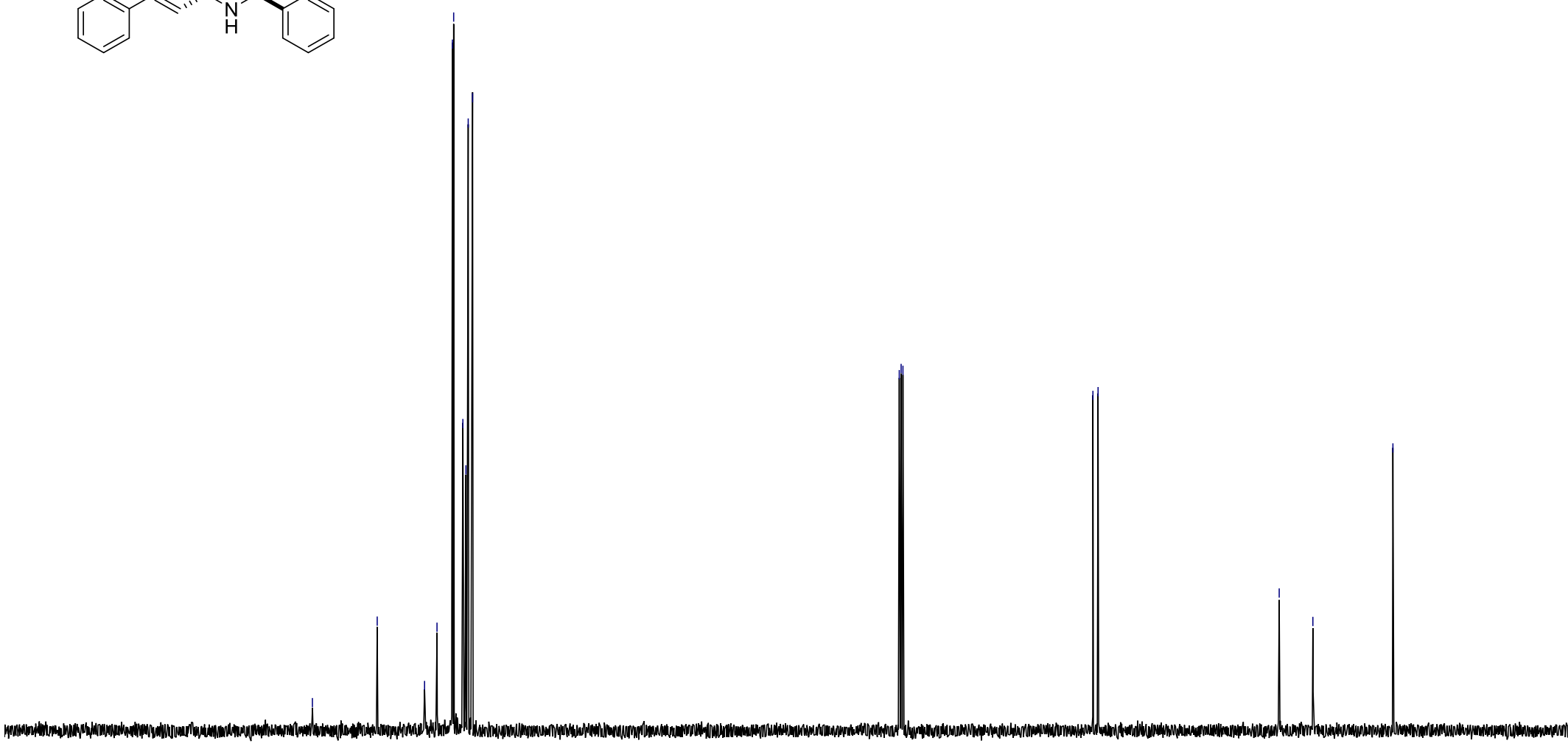

$80 \quad 170 \quad 160$ 150 140 130 120 110 100 90
f1 $(\mathrm{ppm})$ 80 70 60 50 40 30 20 10 


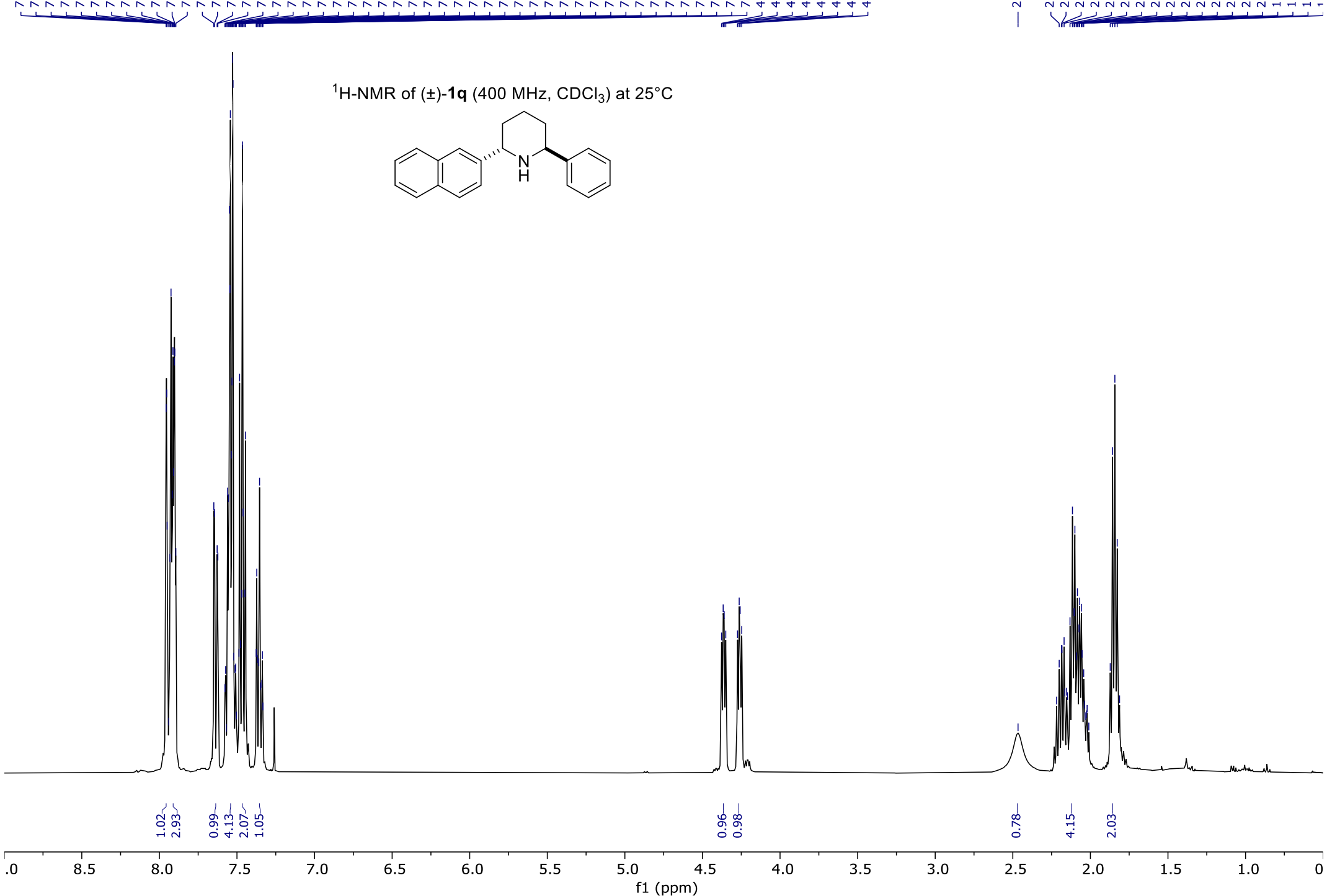


|

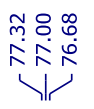

令

mे m m

ลำ

${ }^{13} \mathrm{C}-\mathrm{NMR}$ of $( \pm)-1 \mathrm{q}\left(100 \mathrm{MHz}, \mathrm{CDCl}_{3}\right)$ at $25^{\circ} \mathrm{C}$
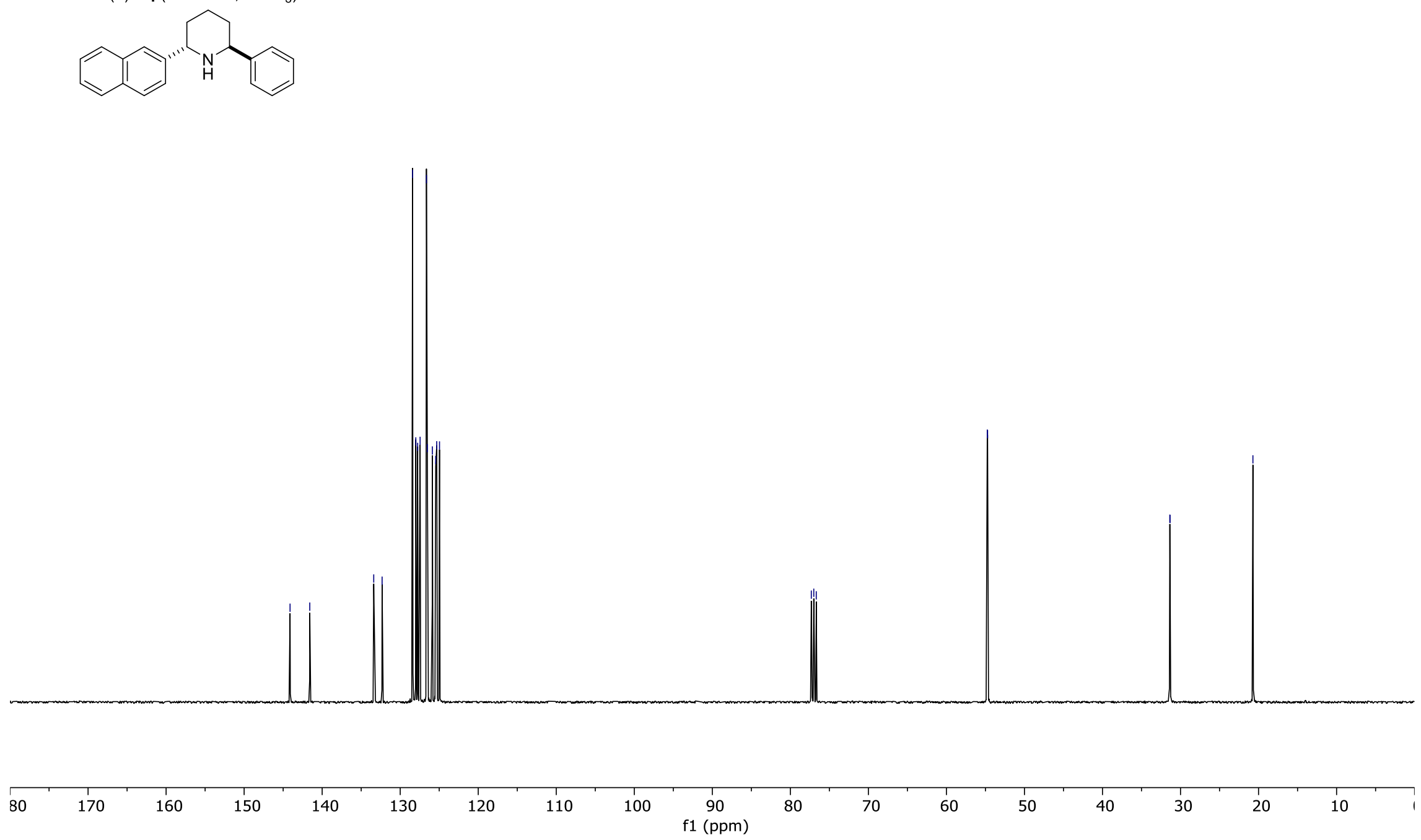

S-110 


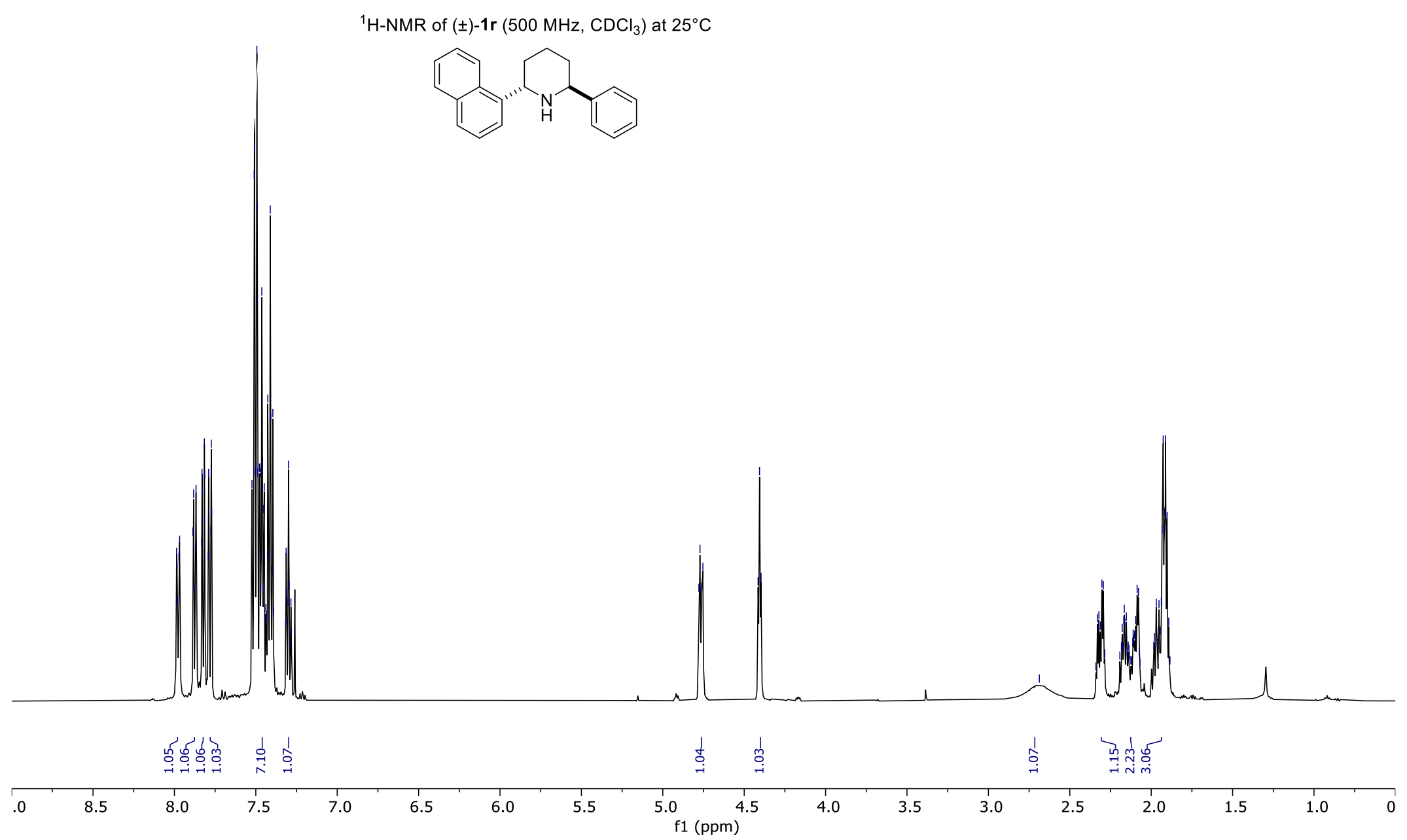

S-111 


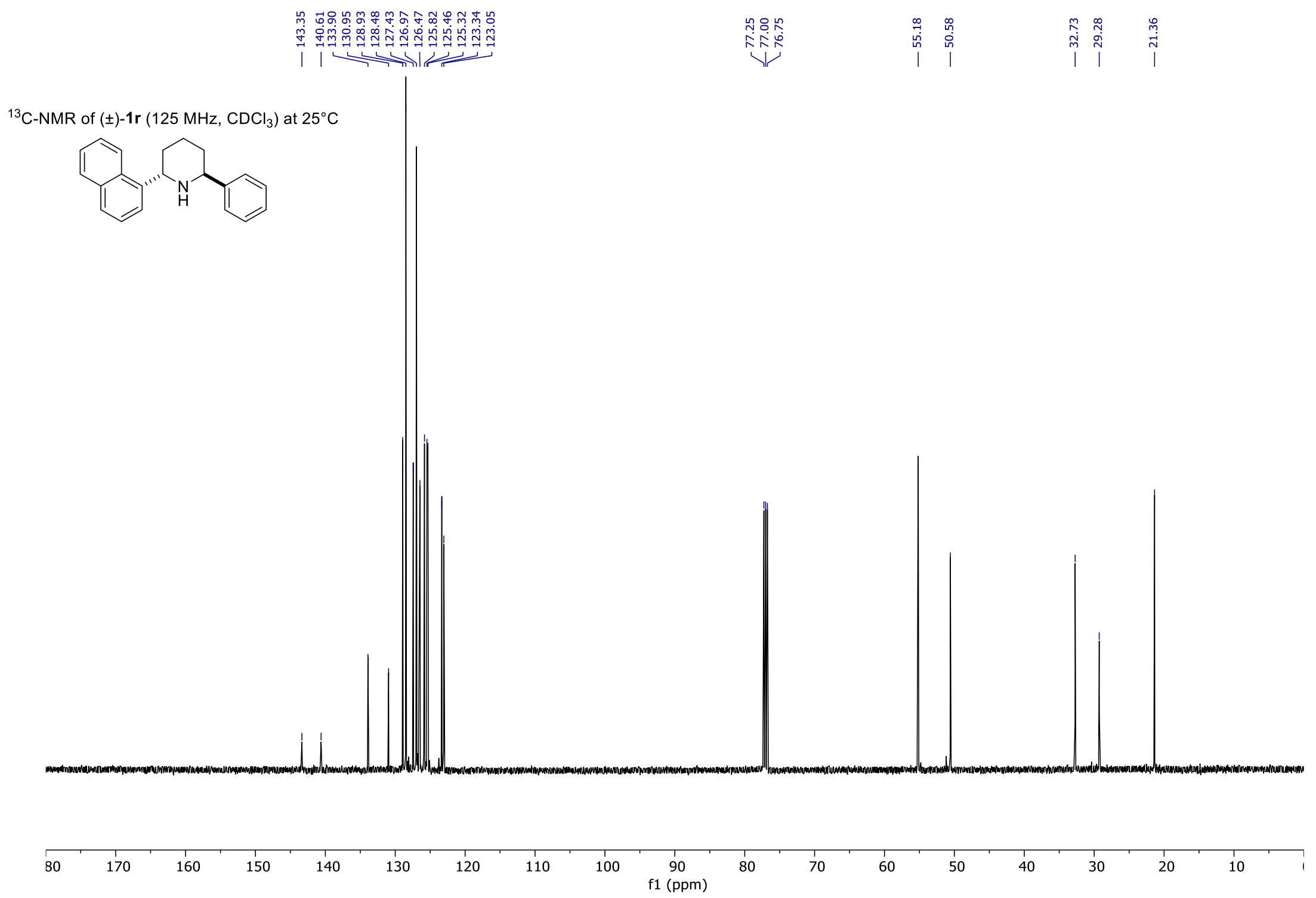

S-112 


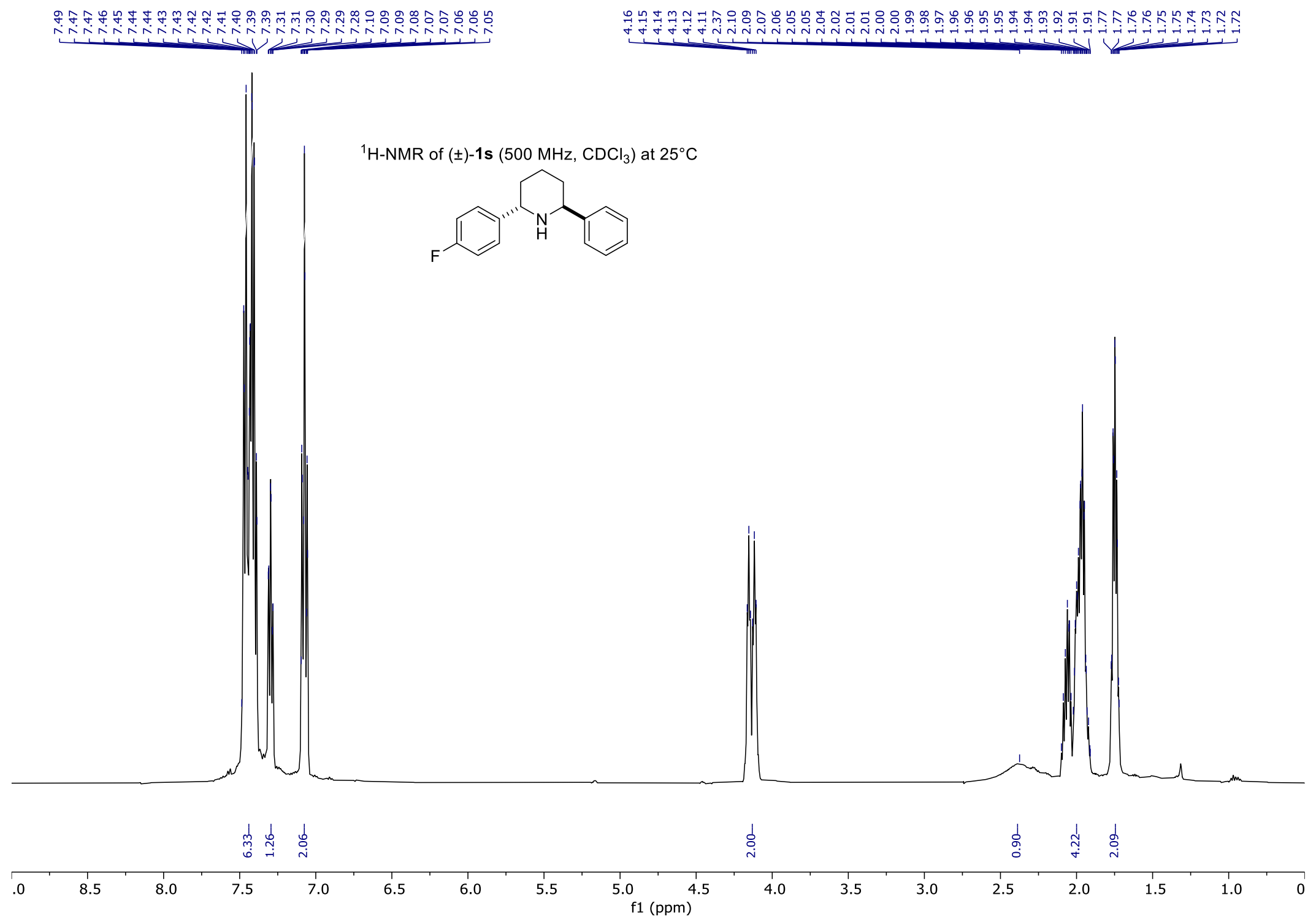

S-113 
${ }^{19} \mathrm{~F}-\mathrm{NMR}$ of $( \pm)-1 \mathrm{~s}\left(470 \mathrm{MHz}, \mathrm{CDCl}_{3}\right)$ at $25^{\circ} \mathrm{C}$

$$
\text { (4) }
$$

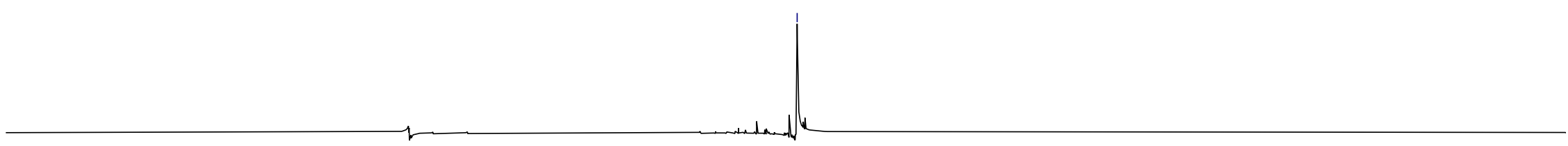




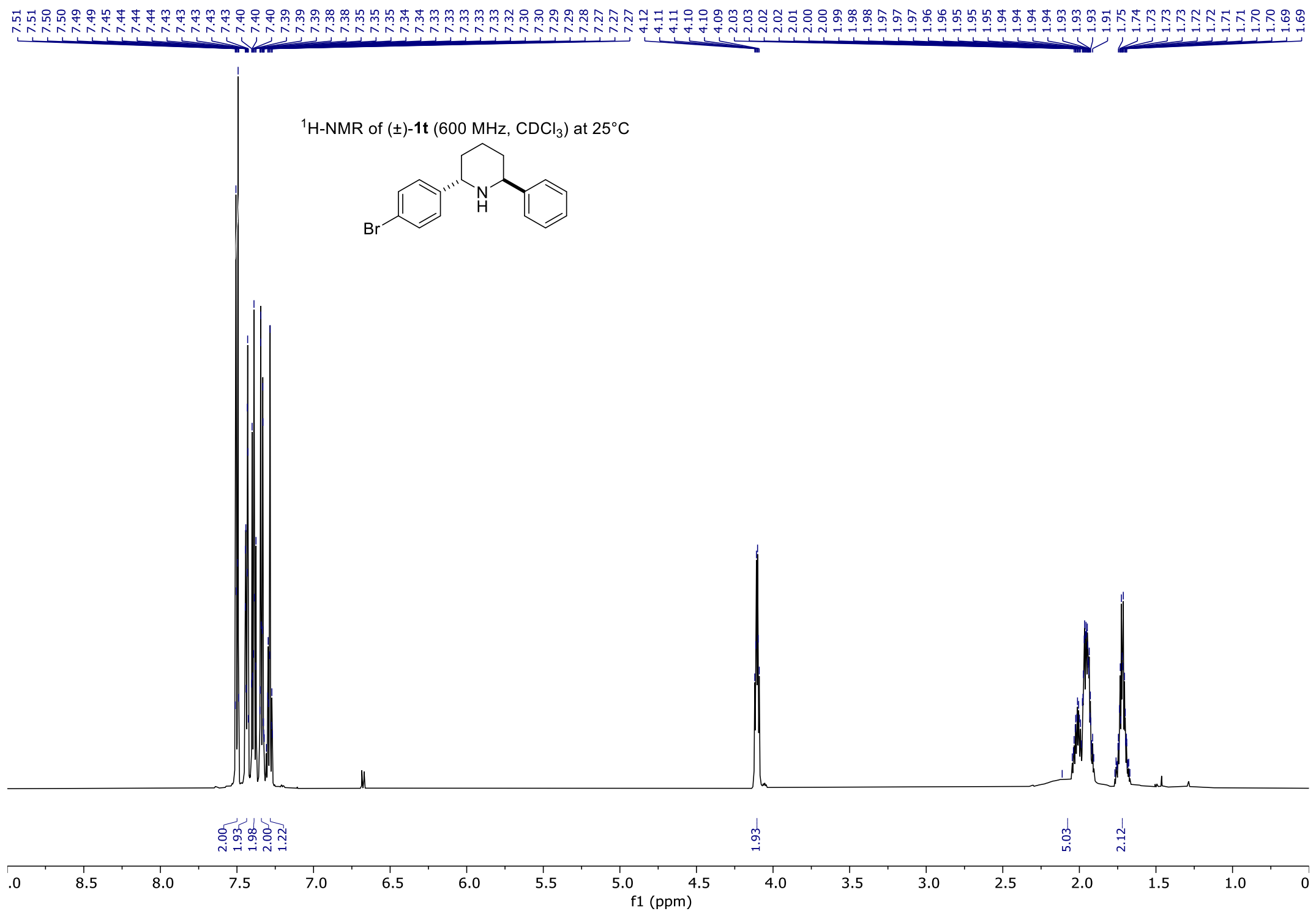

S-116 

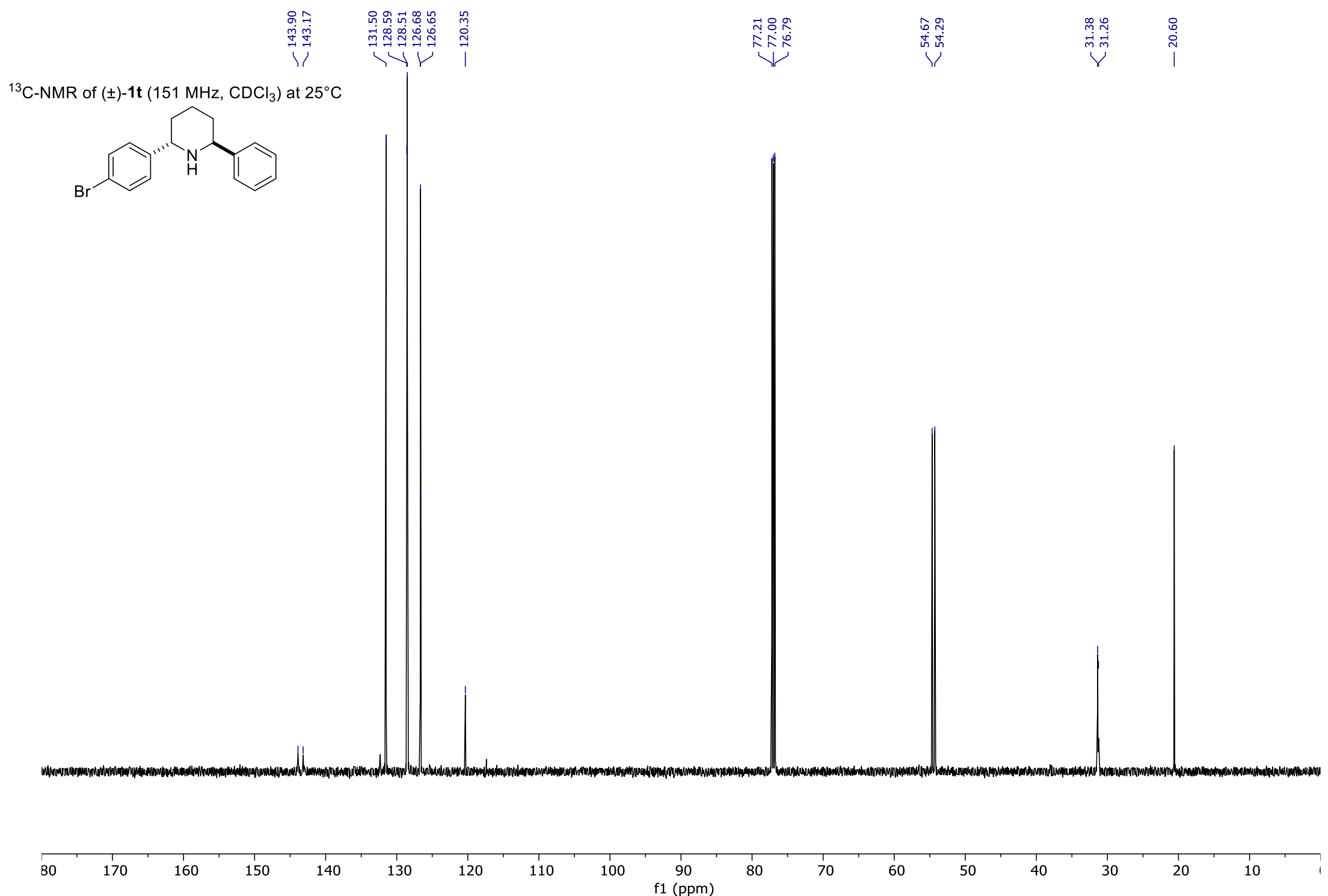

S-117 


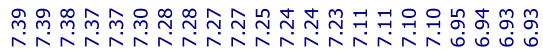

${ }^{1} \mathrm{H}-\mathrm{NMR}$ of $( \pm)-1 \mathrm{u}\left(500 \mathrm{MHz}, \mathrm{CDCl}_{3}\right)$ at $25^{\circ} \mathrm{C}$

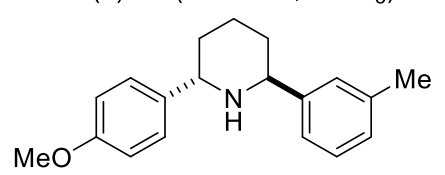

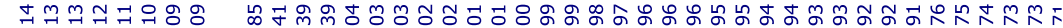

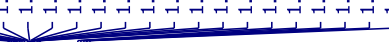

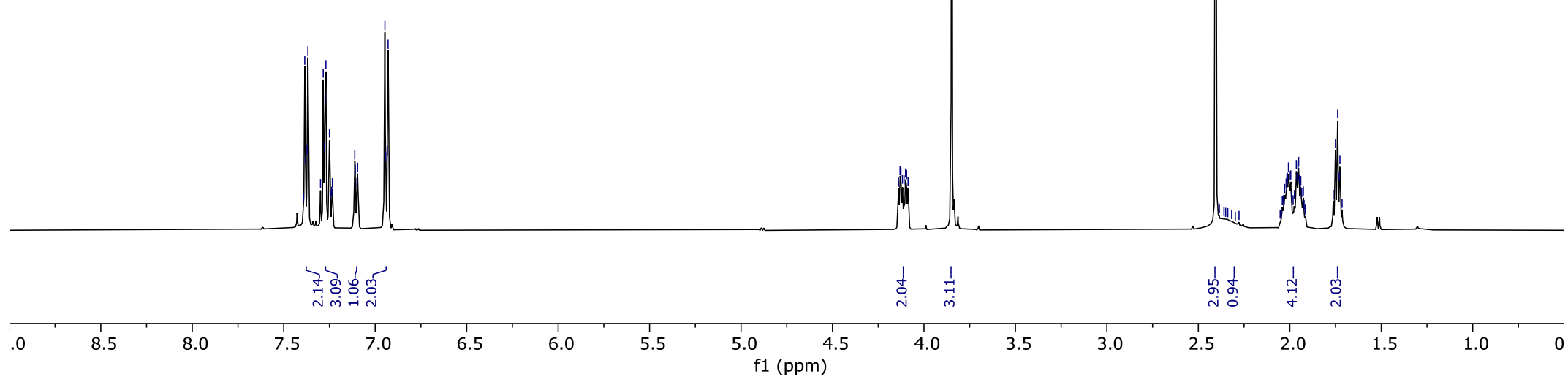

S-118 


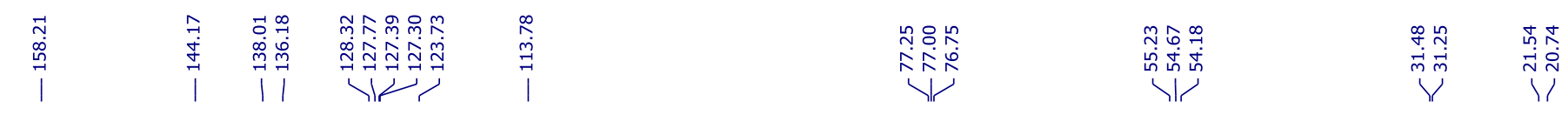

${ }^{13} \mathrm{C}-N M R$ of $( \pm)-1 \mathrm{u}\left(125 \mathrm{MHz}, \mathrm{CDCl}_{3}\right)$ at $25^{\circ} \mathrm{C}$
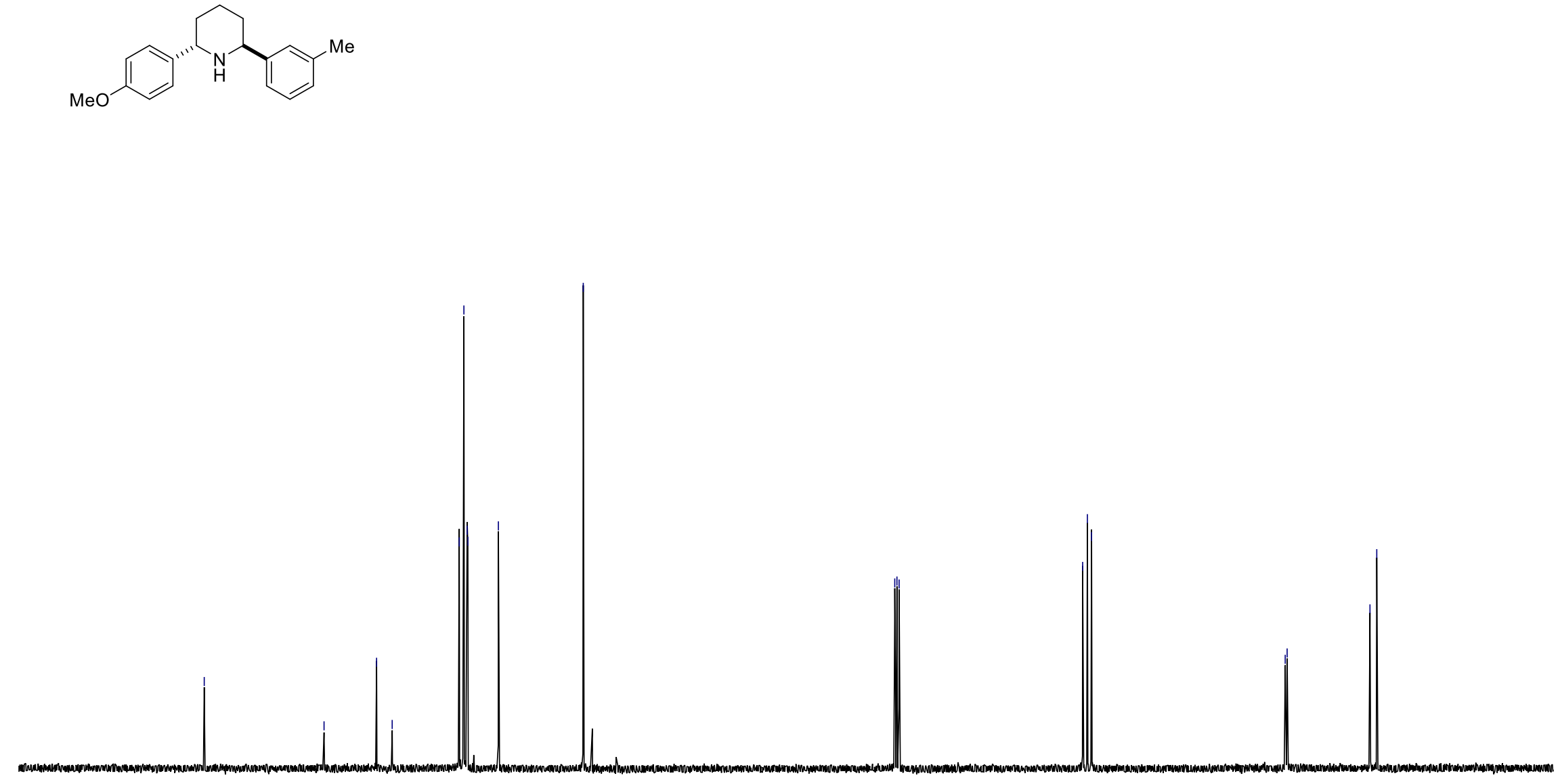


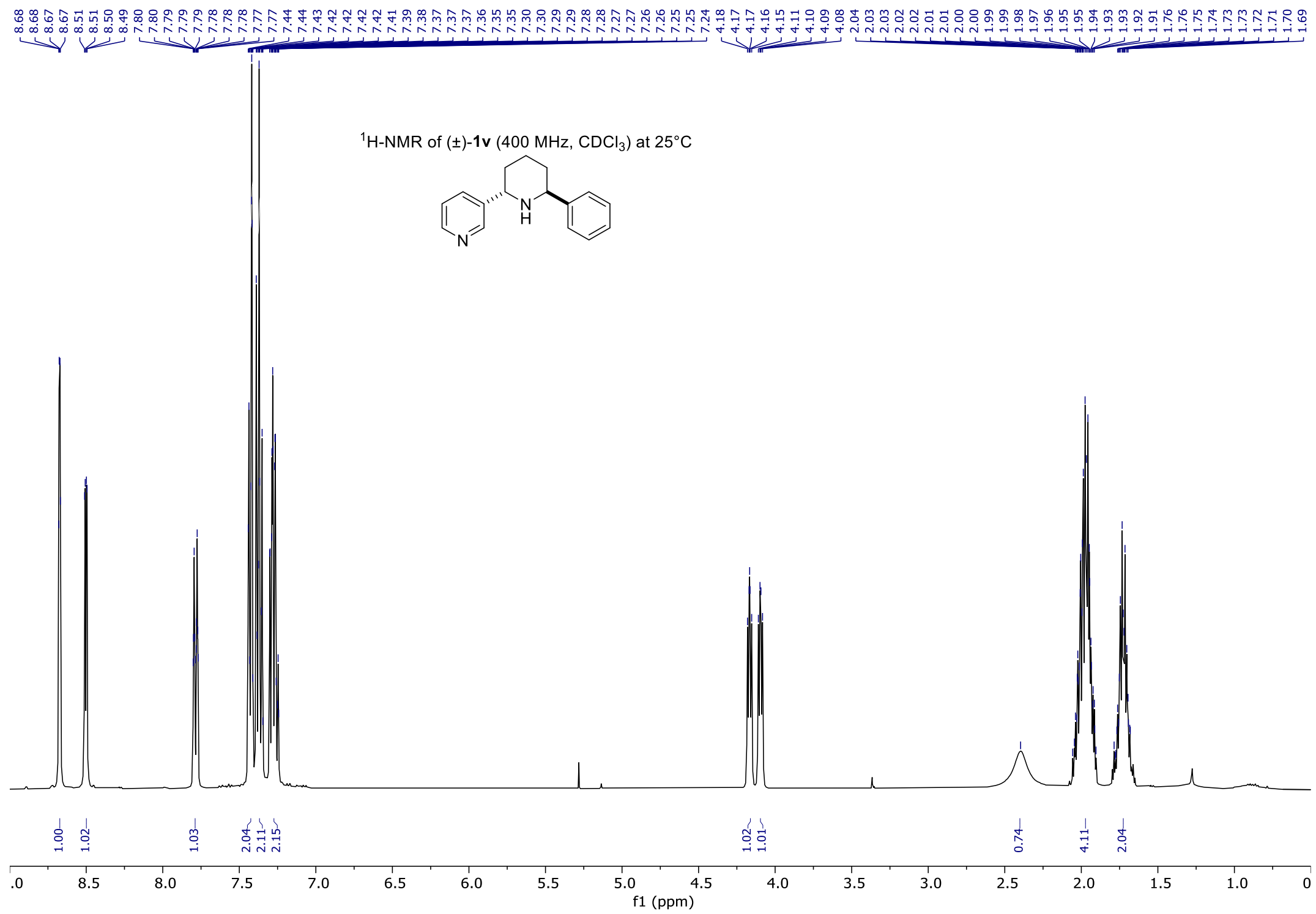

S-120 


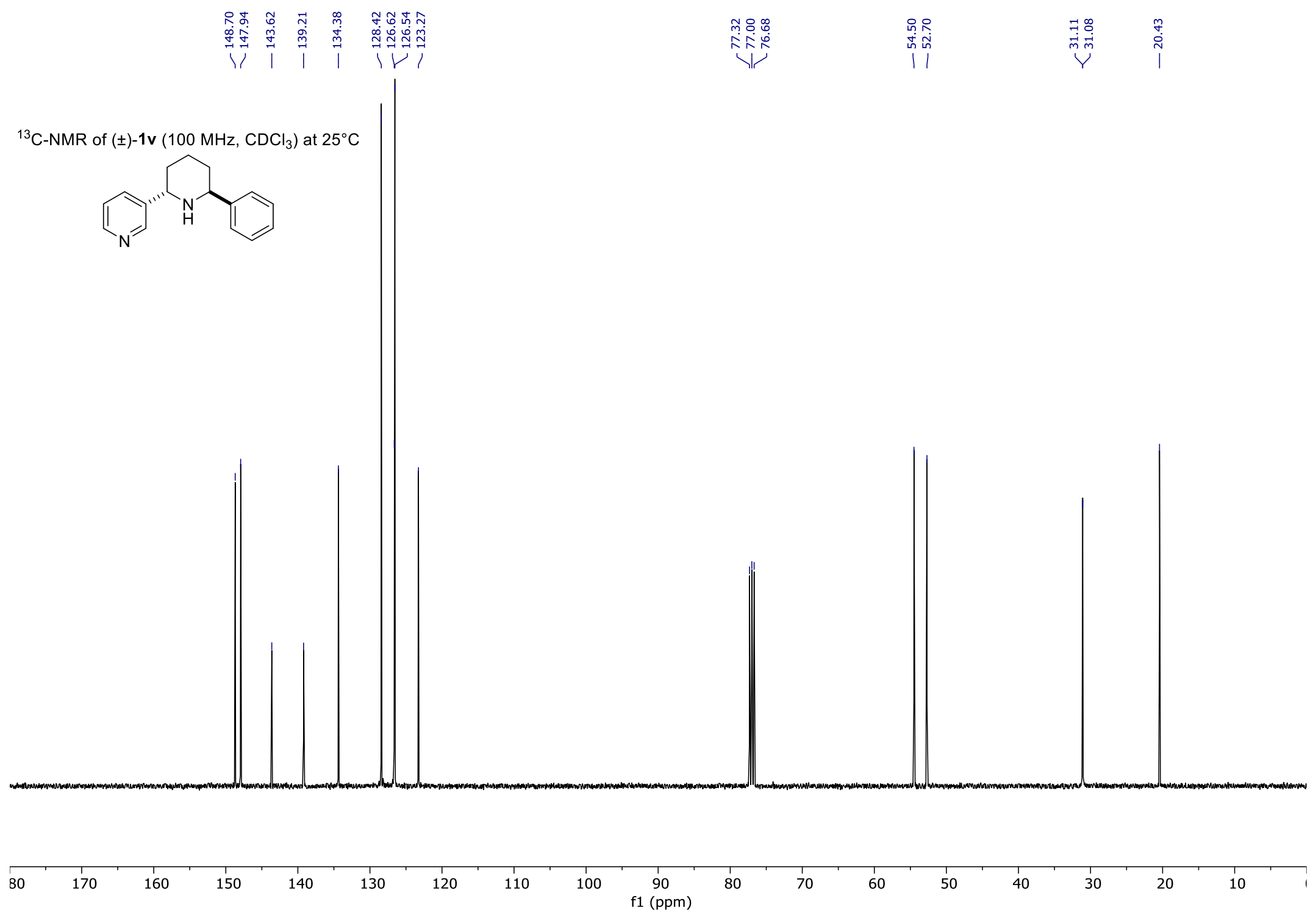

S-121 


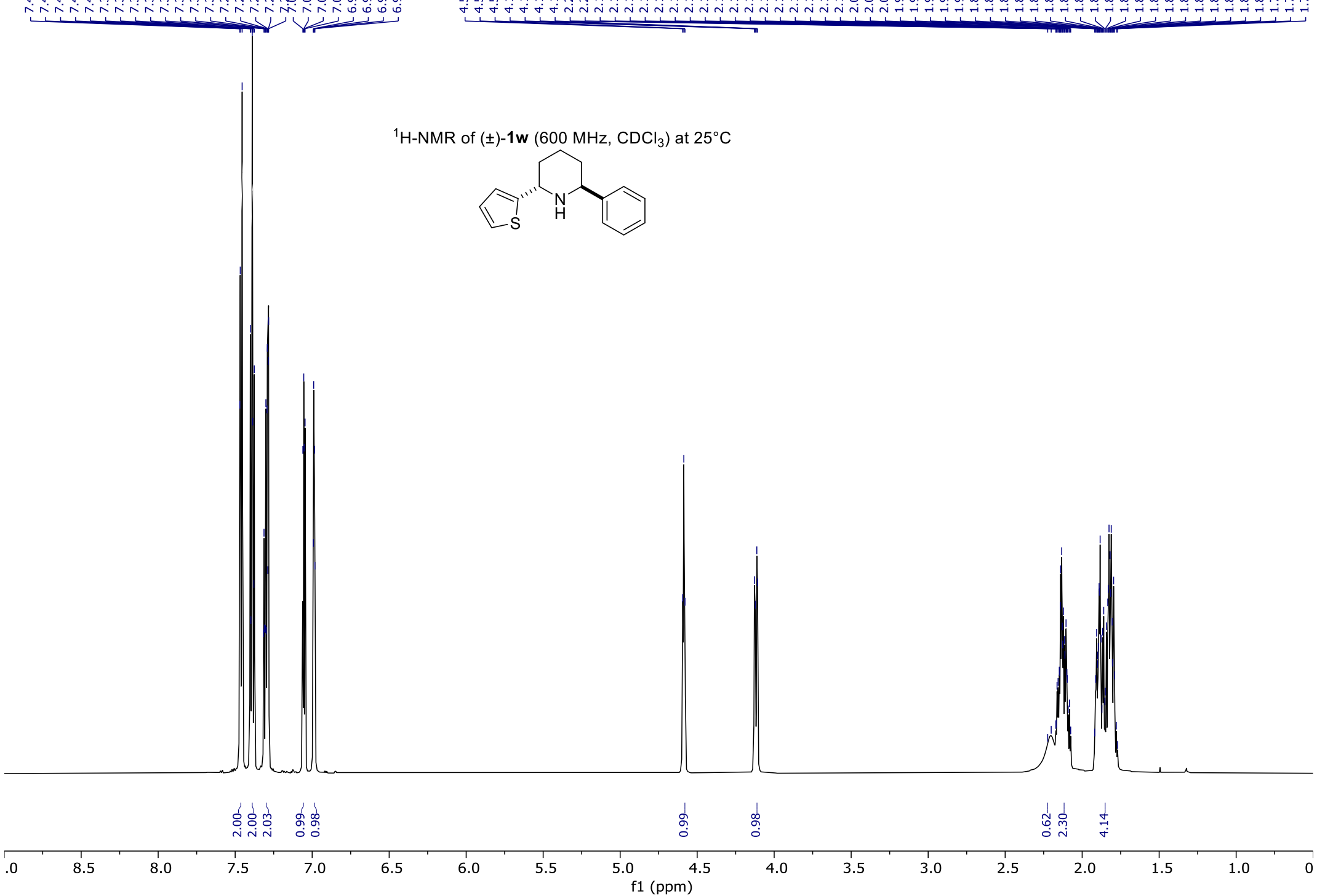

S-122 


$$
\text { | }
$$

${ }^{13} \mathrm{C}-\mathrm{NMR}$ of $( \pm)-1 \mathrm{w}\left(150 \mathrm{MHz}, \mathrm{CDCl}_{3}\right)$ at $25^{\circ} \mathrm{C}$<smiles></smiles>

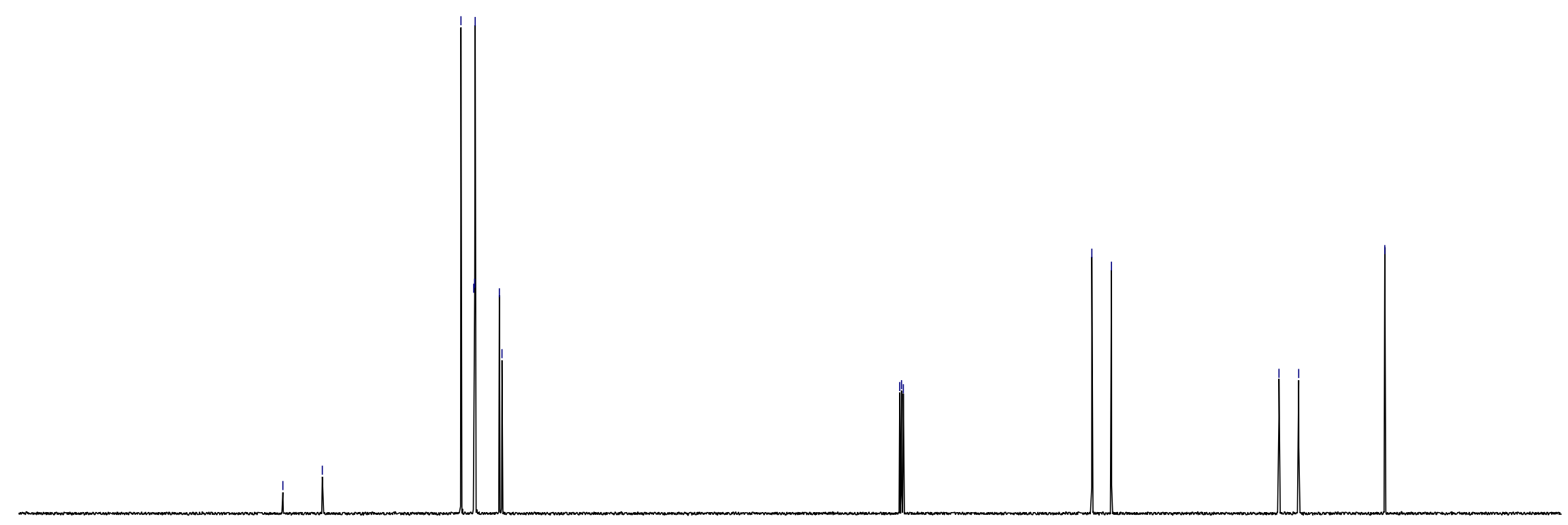




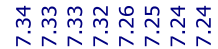

rivivinising

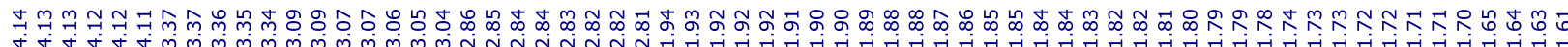

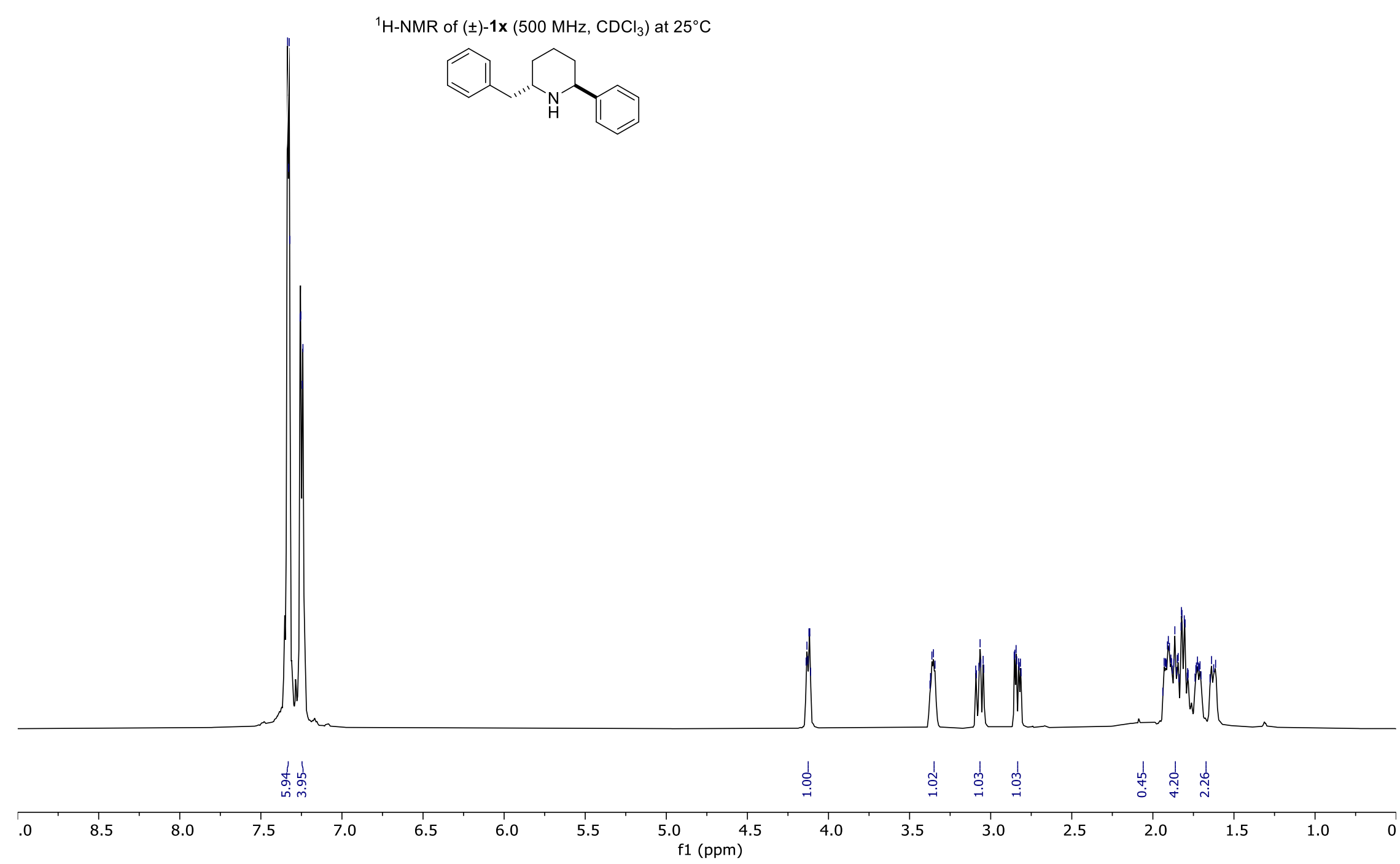

S-124 


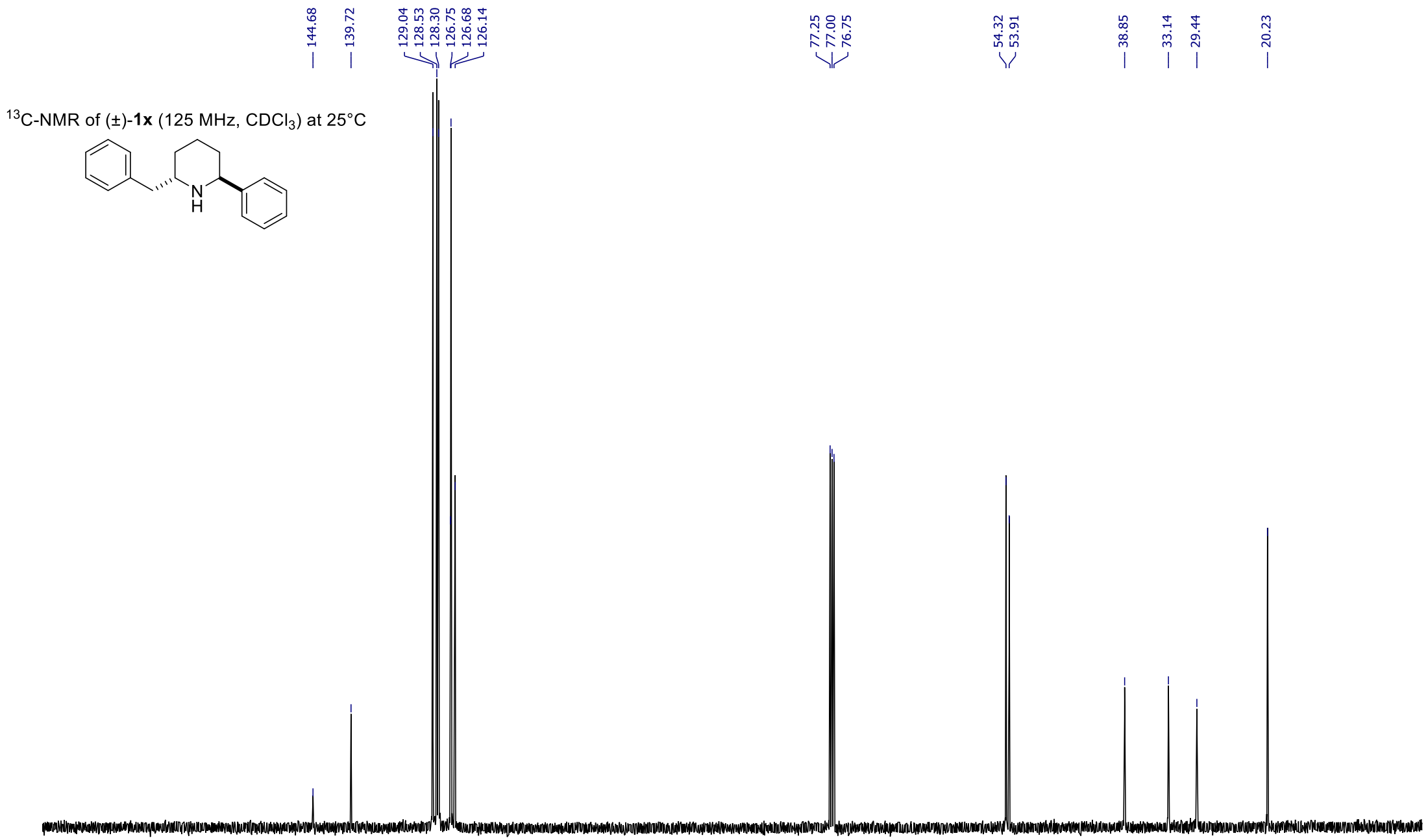

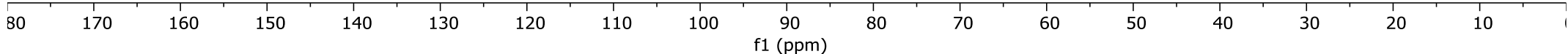




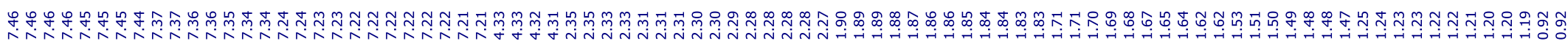

${ }^{1} \mathrm{H}-\mathrm{NMR}$ of $( \pm)-1 \mathrm{y}\left(500 \mathrm{MHz}, \mathrm{CDCl}_{3}\right)$ at $25^{\circ} \mathrm{C}$<smiles>CC(C)(C)[C@H]1CCC[C@H](c2ccccc2)N1</smiles>

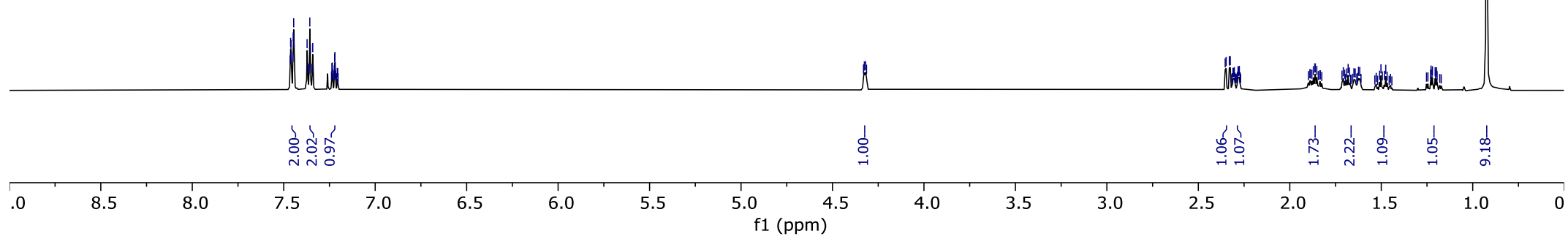

S-126 


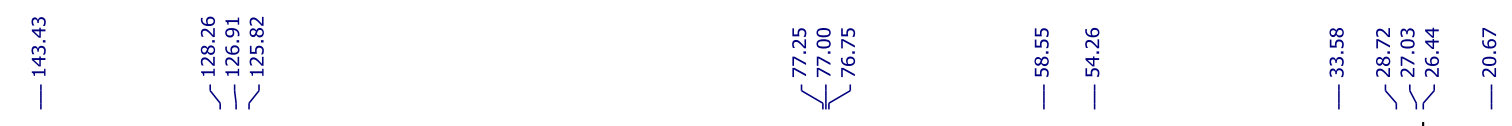

${ }^{13} \mathrm{C}-\mathrm{NMR}$ of $( \pm)-1 \mathrm{y}\left(125 \mathrm{MHz}, \mathrm{CDCl}_{3}\right)$ at $25^{\circ} \mathrm{C}$
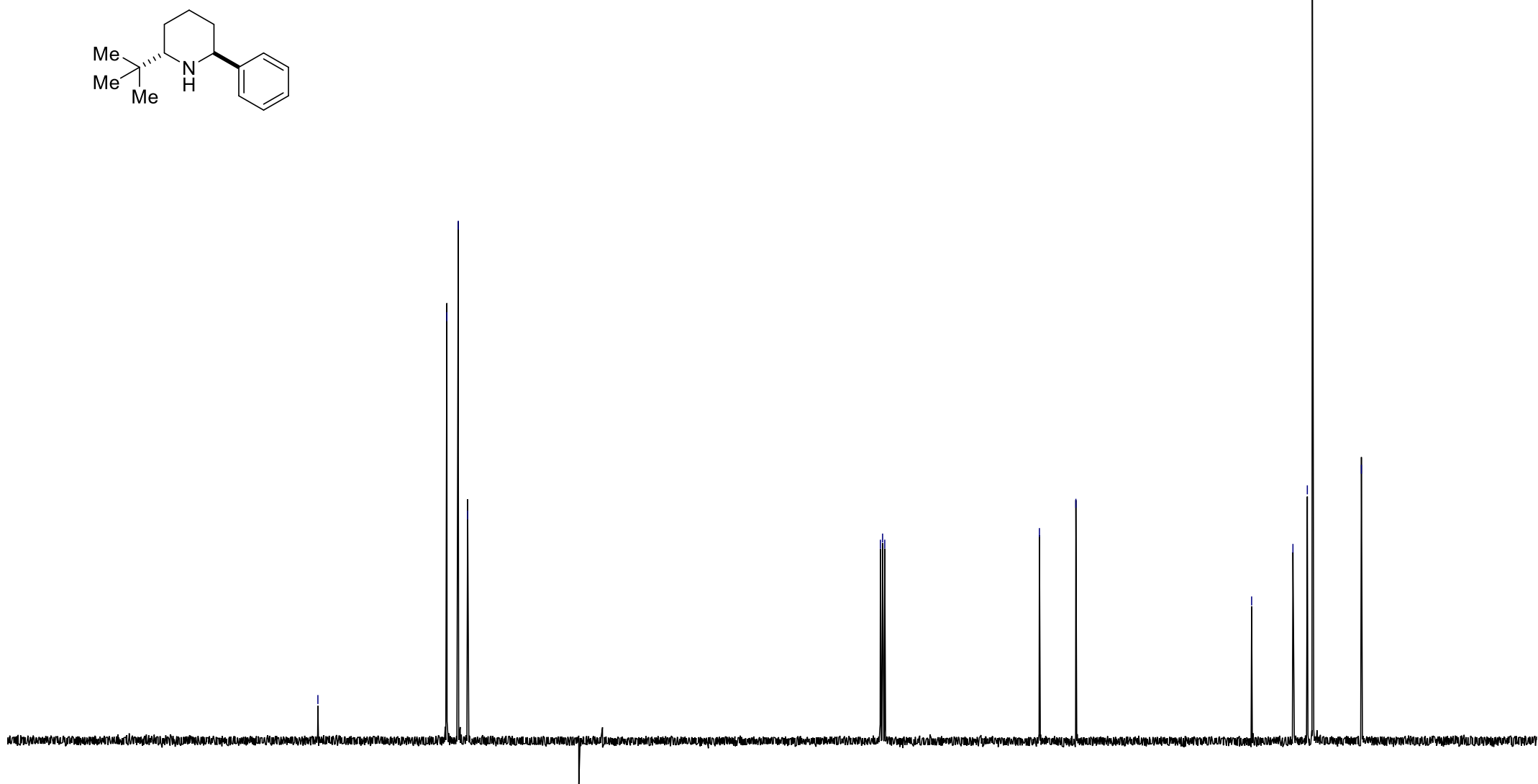

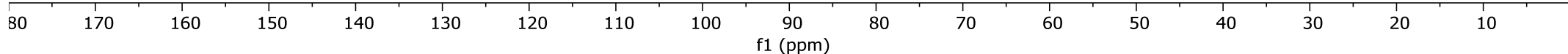




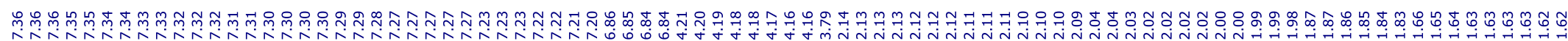

${ }^{1} \mathrm{H}-\mathrm{NMR}$ of $( \pm)-1 \mathrm{z}\left(400 \mathrm{MHz}, \mathrm{CDCl}_{3}\right)$ at $25^{\circ} \mathrm{C}$
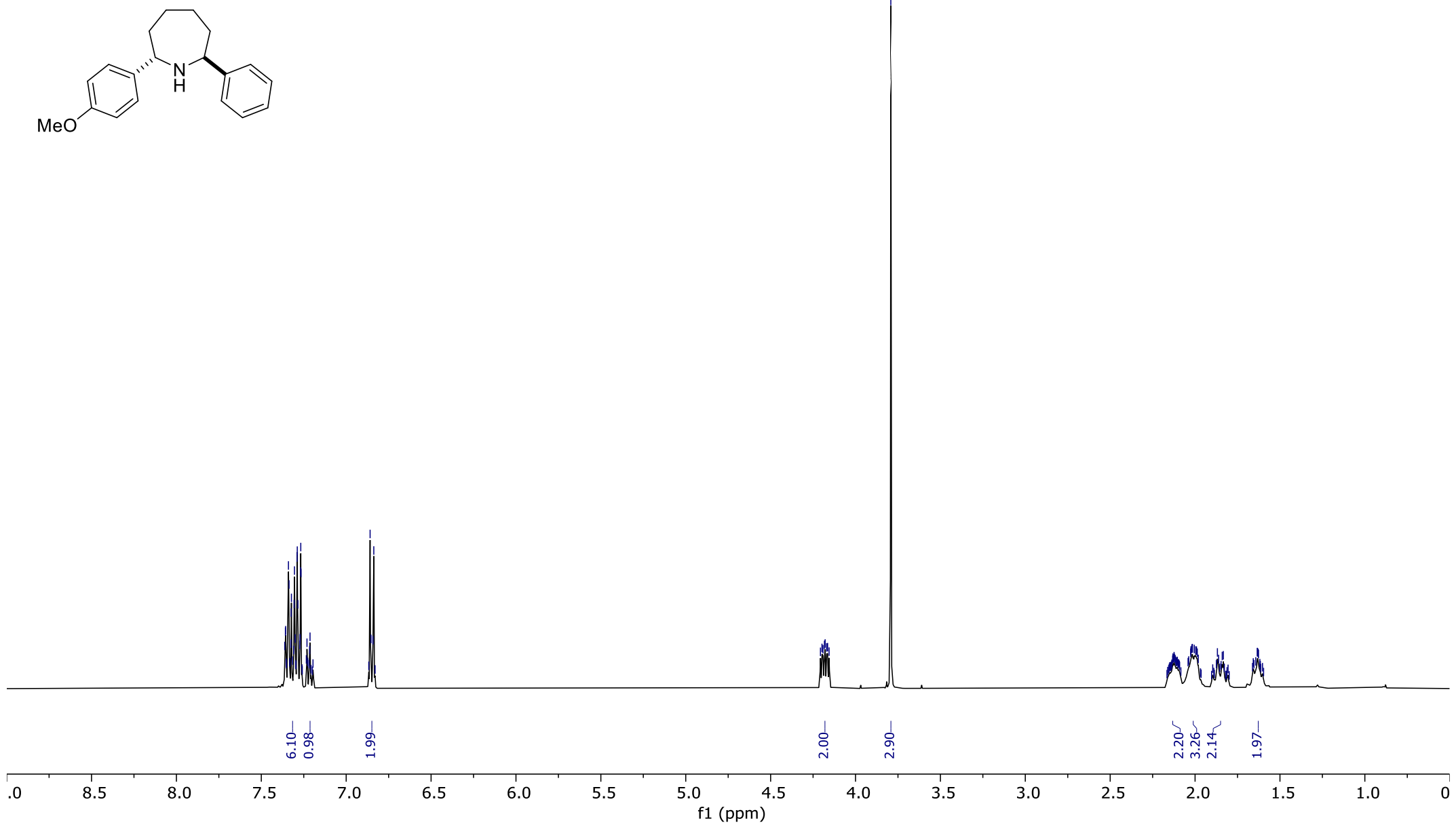


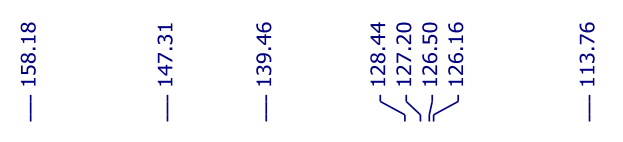

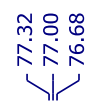

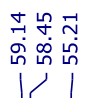

$\bigcup_{\substack{0 \\ \infty}}^{\substack{d \\ \infty}}$

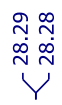

${ }^{13} \mathrm{C}-N M R$ of $( \pm)-1 \mathrm{z}\left(100 \mathrm{MHz}, \mathrm{CDCl}_{3}\right)$ at $25^{\circ} \mathrm{C}$
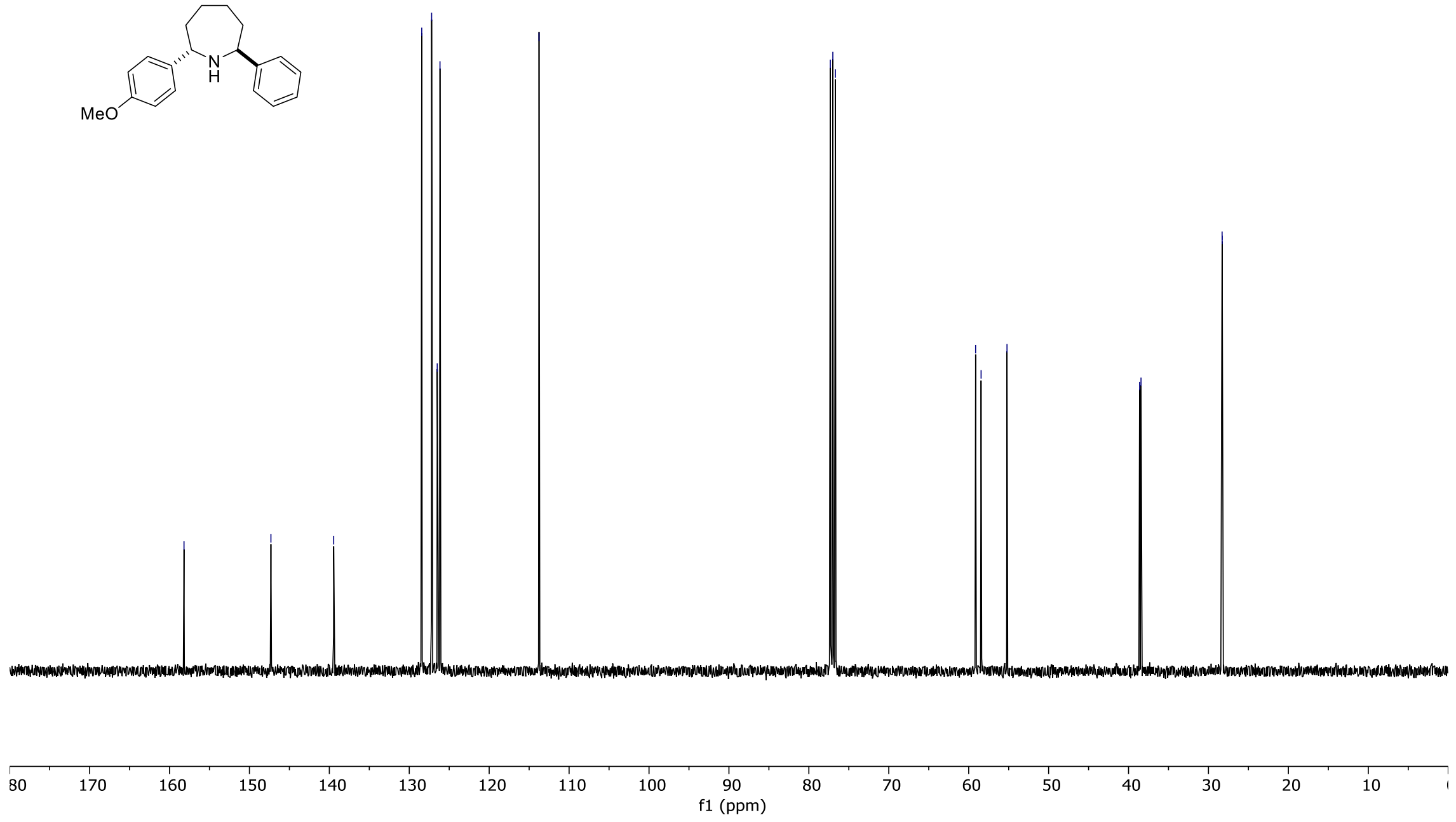

S-129 
<smiles>CCCCC1CCCC[C@H](CCCC)N1</smiles>

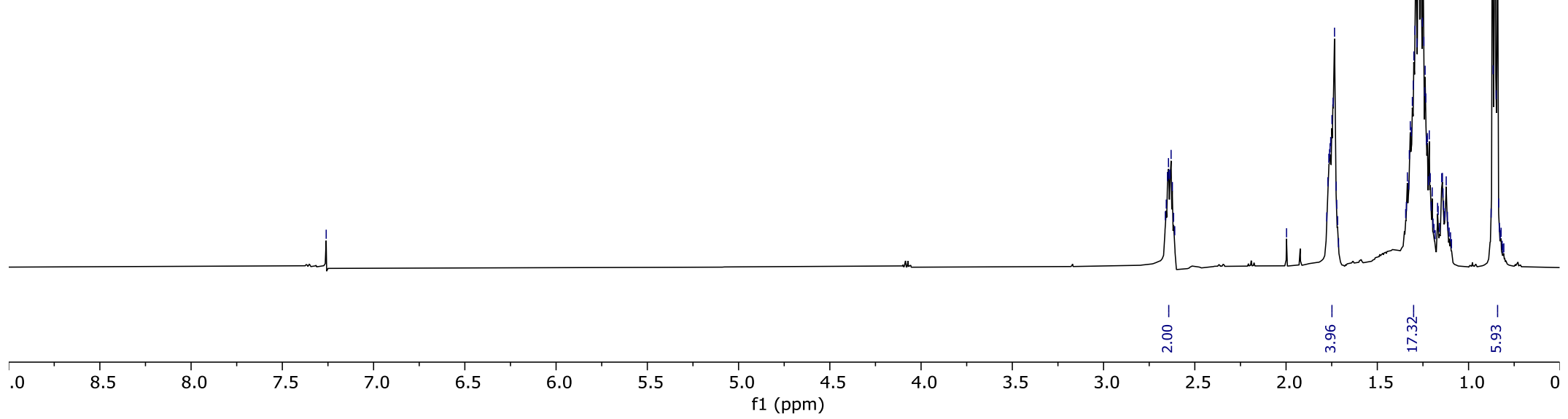

S-130 
${ }^{13} \mathrm{C}-\mathrm{NMR}$ of $( \pm)-1$ aa $\left(125 \mathrm{MHz}, \mathrm{CDCl}_{3}\right)$ at $25^{\circ} \mathrm{C}$<smiles>CCCC[C@H]1CCCC[C@H](CCCC)N1</smiles> 


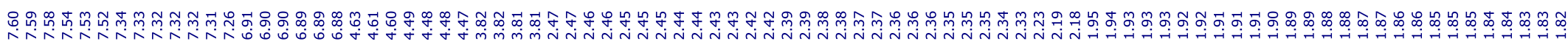

${ }^{1} \mathrm{H}-\mathrm{NMR}$ of $( \pm)-1 \mathbf{a b}\left(500 \mathrm{MHz}, \mathrm{CDCl}_{3}\right)$ at $25^{\circ} \mathrm{C}$<smiles>COc1ccc(C2CC[C@@H](c3ccc(C(F)(F)F)cc3)N2)cc1</smiles>

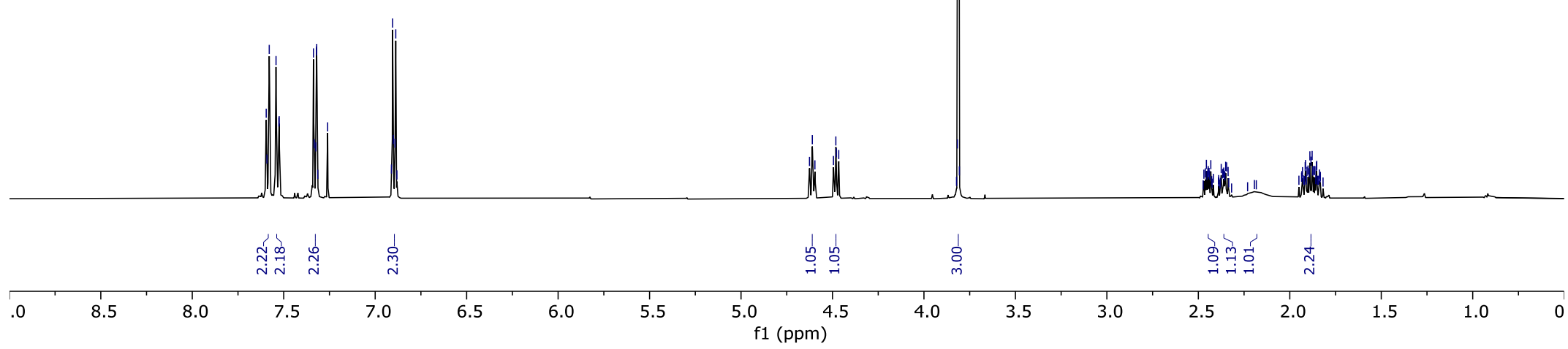




$$
\begin{array}{llll}
0 & 0 \\
0 & 0 & 0
\end{array}
$$

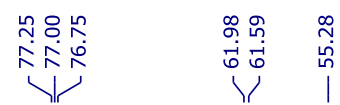

Vִ

${ }^{13} \mathrm{C}-\mathrm{NMR}$ of $( \pm)-\mathbf{1 a b}\left(125 \mathrm{MHz}, \mathrm{CDCl}_{3}\right)$ at $25^{\circ} \mathrm{C}$<smiles>COc1ccc(C2CC[C@H](c3ccc(C(F)(F)F)cc3)N2)cc1</smiles>

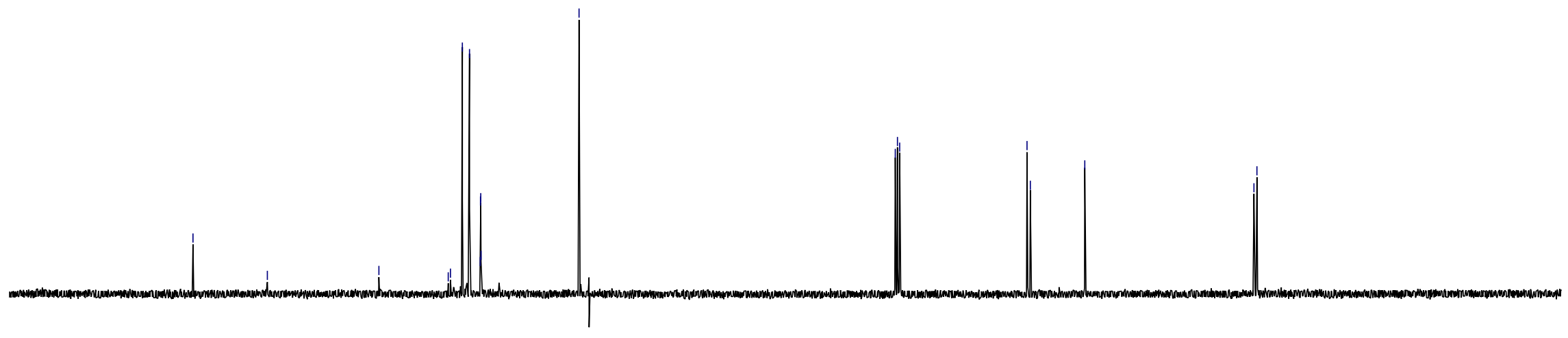


${ }^{19} \mathrm{~F}-\mathrm{NMR}$ of $( \pm)-\mathbf{1 a b}\left(470 \mathrm{MHz}, \mathrm{CDCl}_{3}\right)$ at $25^{\circ} \mathrm{C}$

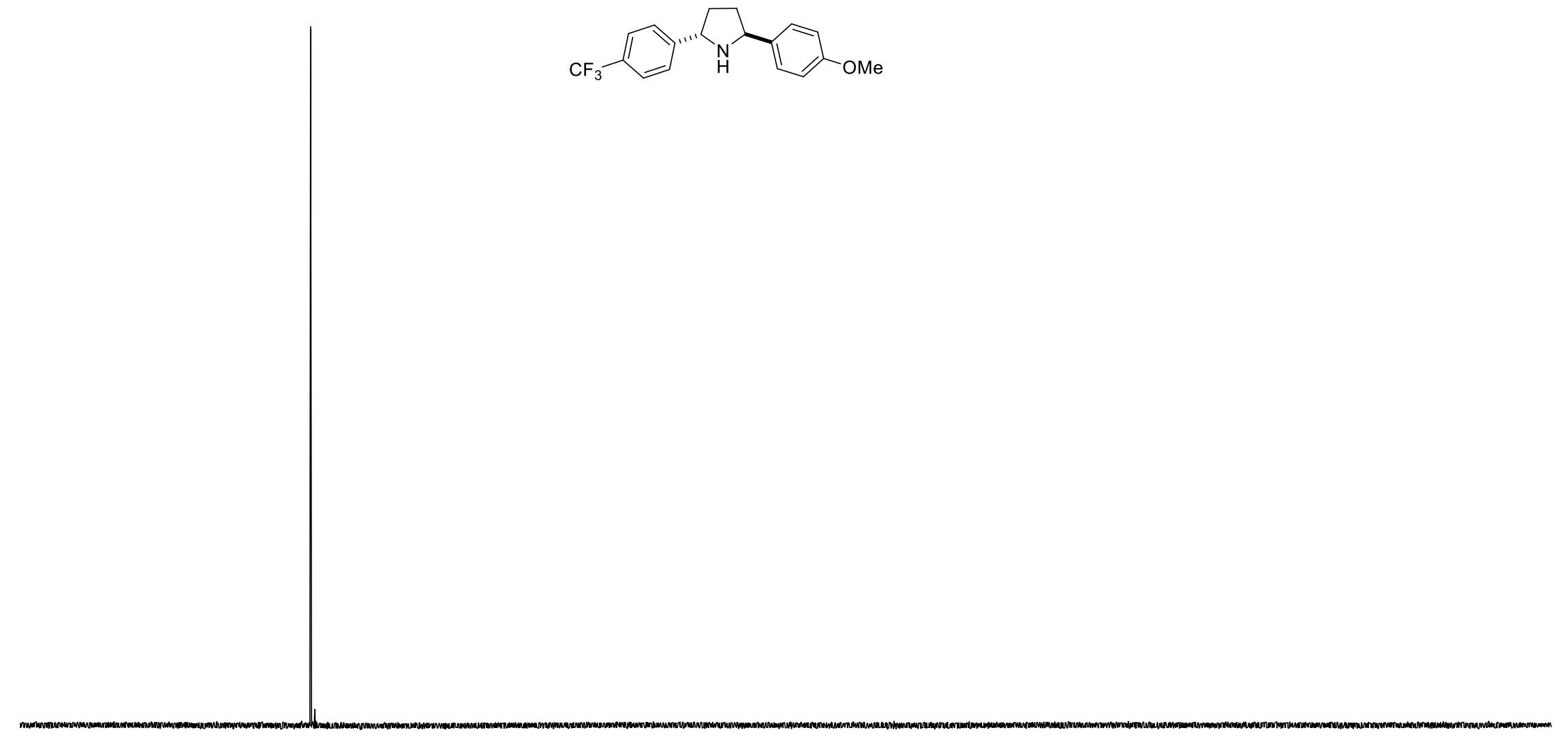


${ }^{1} \mathrm{H}-\mathrm{NMR}$ of $( \pm)-1 \mathrm{ac}\left(600 \mathrm{MHz}, \mathrm{CDCl}_{3}\right)$ at $25^{\circ} \mathrm{C}$<smiles>Cc1cccc(C2CC[C@@H](c3ccc(Br)cc3)N2)c1</smiles>

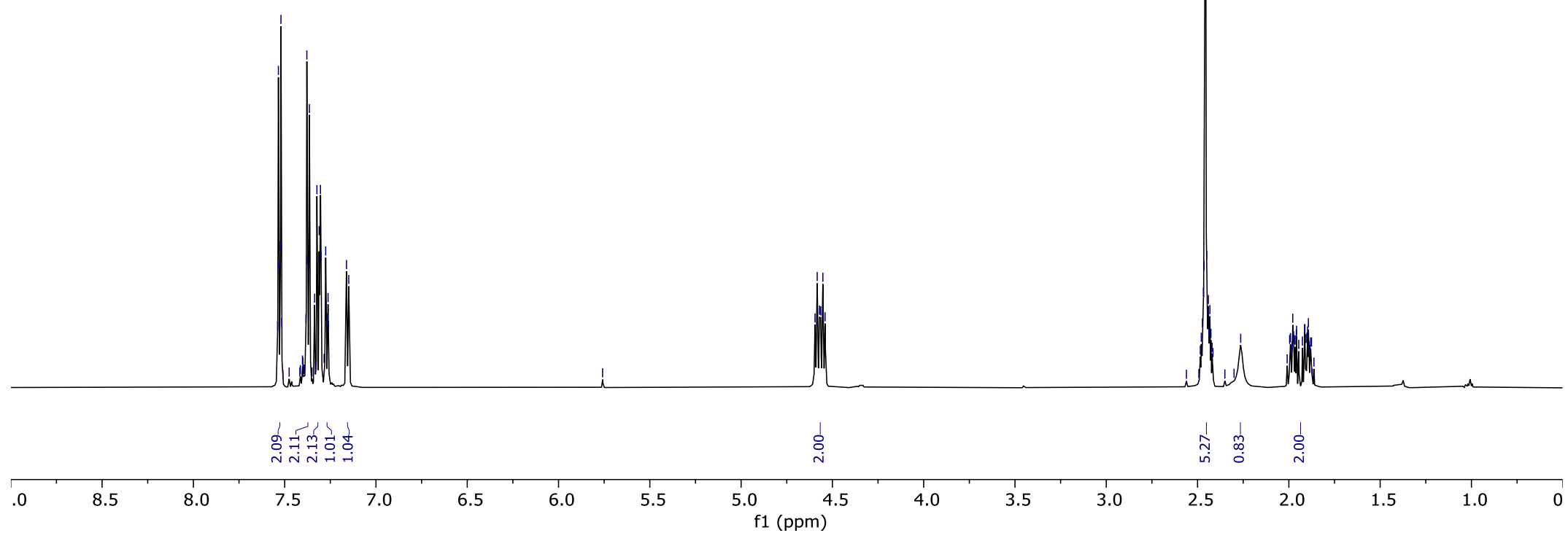

S-135 


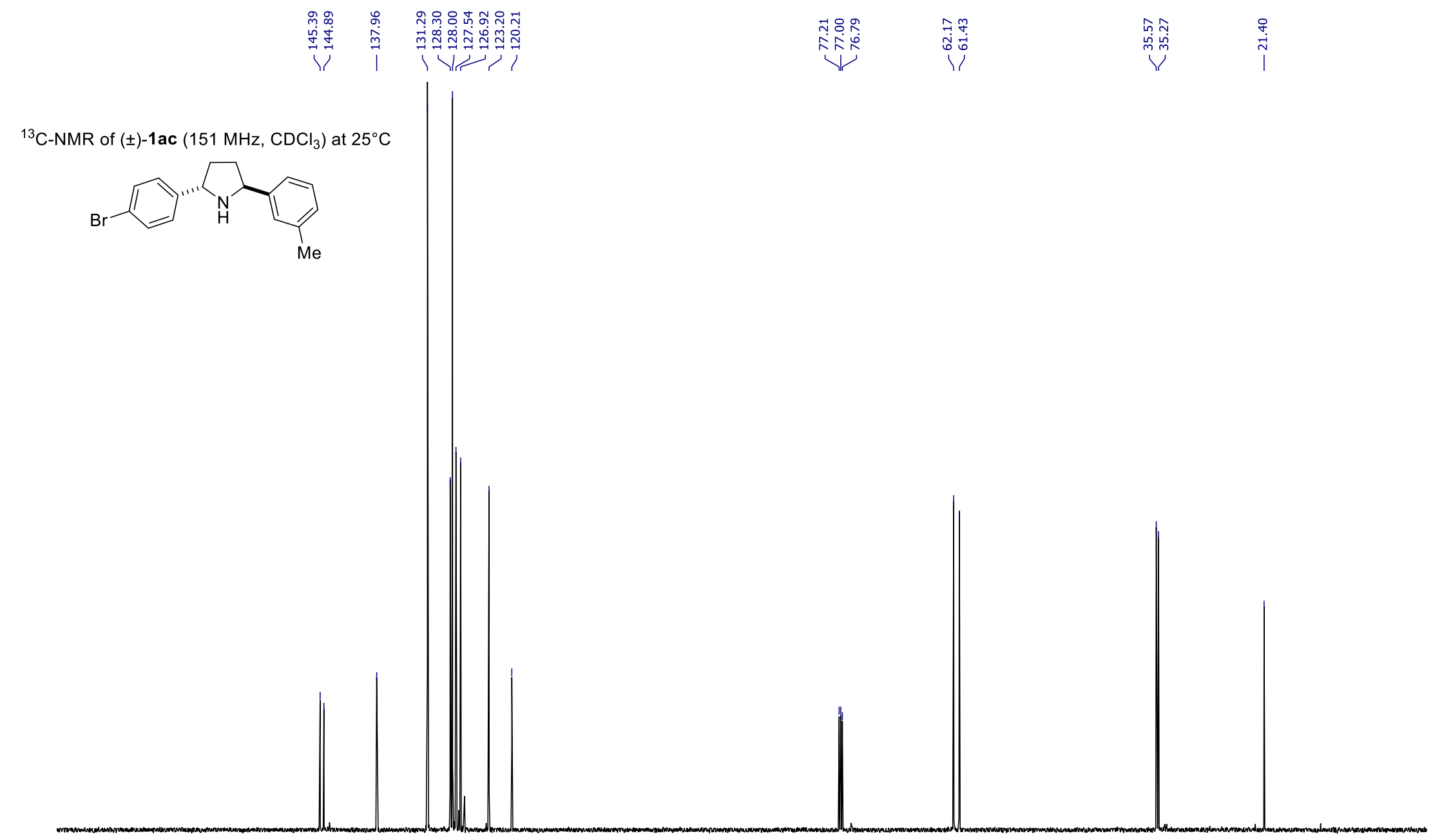

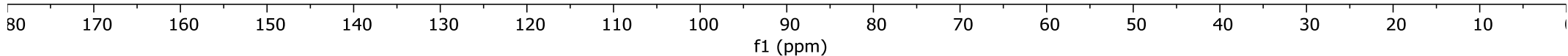

S-136 


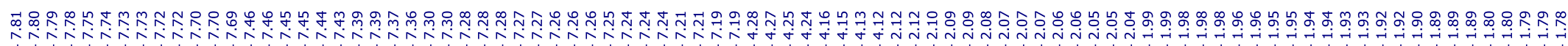

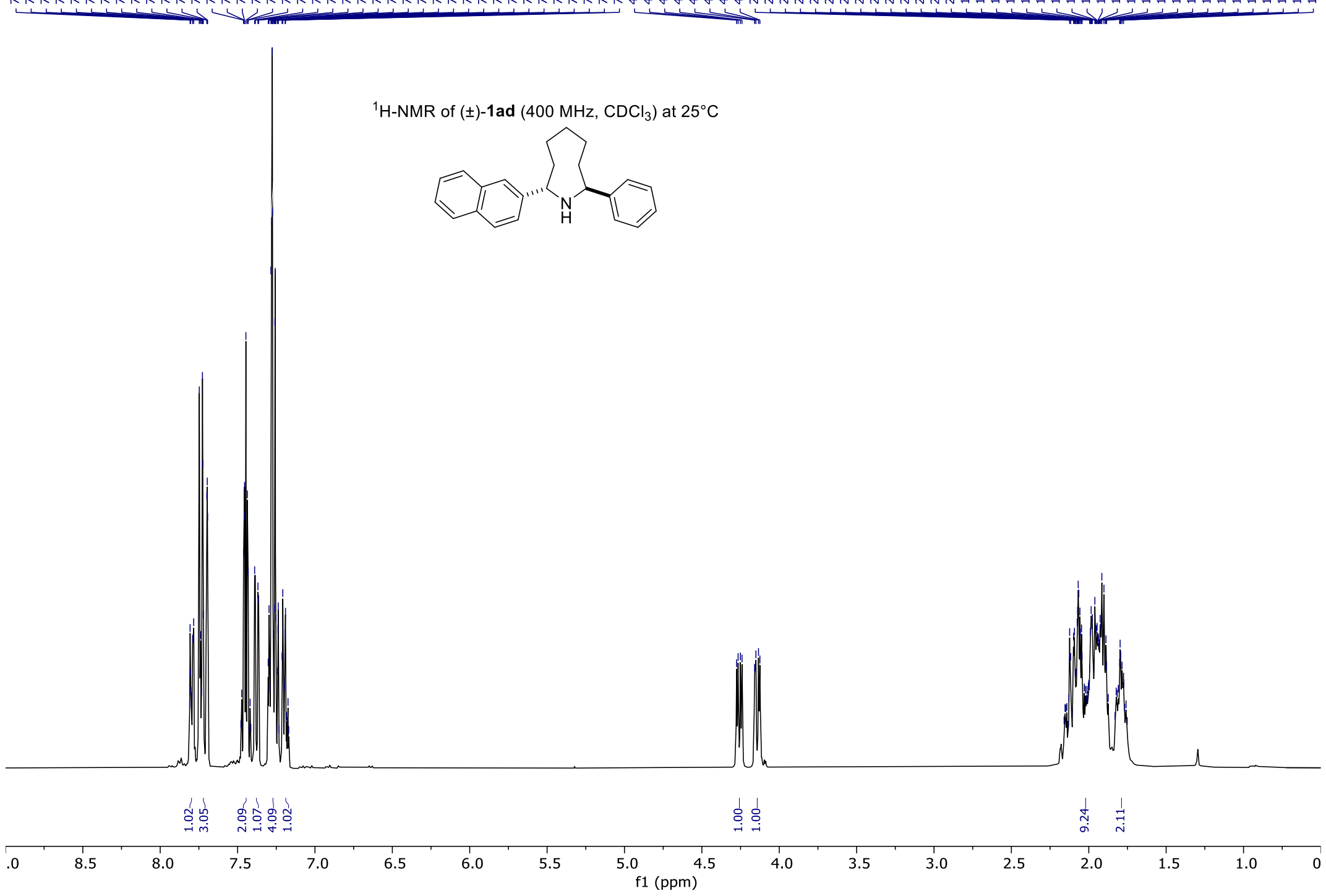

S-137 


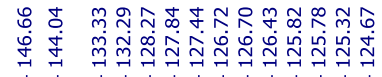

1 年

${ }^{13} \mathrm{C}-\mathrm{NMR}$ of $( \pm)-\mathbf{1 a d}\left(100 \mathrm{MHz}, \mathrm{CDCl}_{3}\right)$ at $25^{\circ} \mathrm{C}$<smiles>c1ccc(C2CCCCC[C@@H](c3ccc4ccccc4c3)N2)cc1</smiles>

mุi

슈

$1 /$

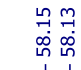

Y

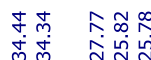

บำ

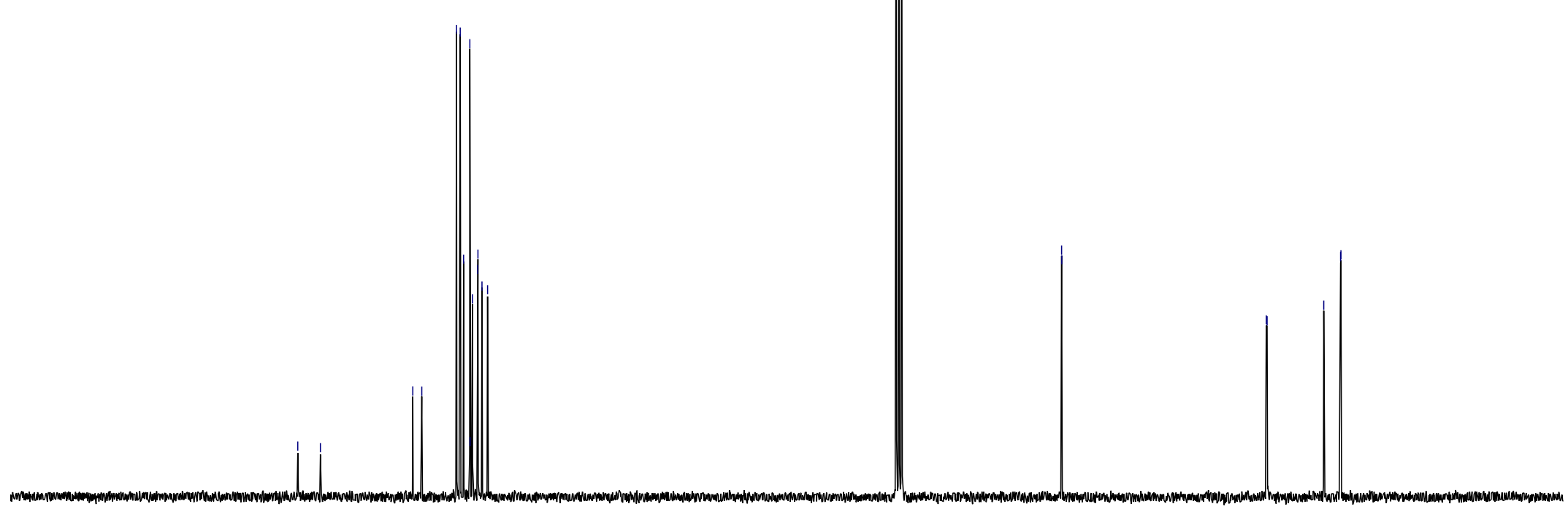

80 $170 \quad 160$ 150 140 130 120 110 100 90
$\mathrm{f} 1(\mathrm{ppm})$ 80 70 60 50 40 30 


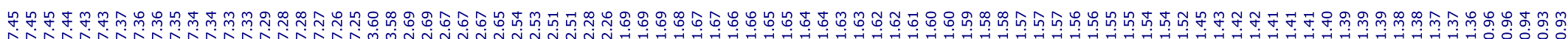

${ }^{1} \mathrm{H}-\mathrm{NMR}$ of $( \pm)-1$ ae $\left(400 \mathrm{MHz}, \mathrm{CDCl}_{3}\right)$ at $25^{\circ} \mathrm{C}$<smiles>CCCCC1NC(c2ccccc2)C2CCCC12</smiles>

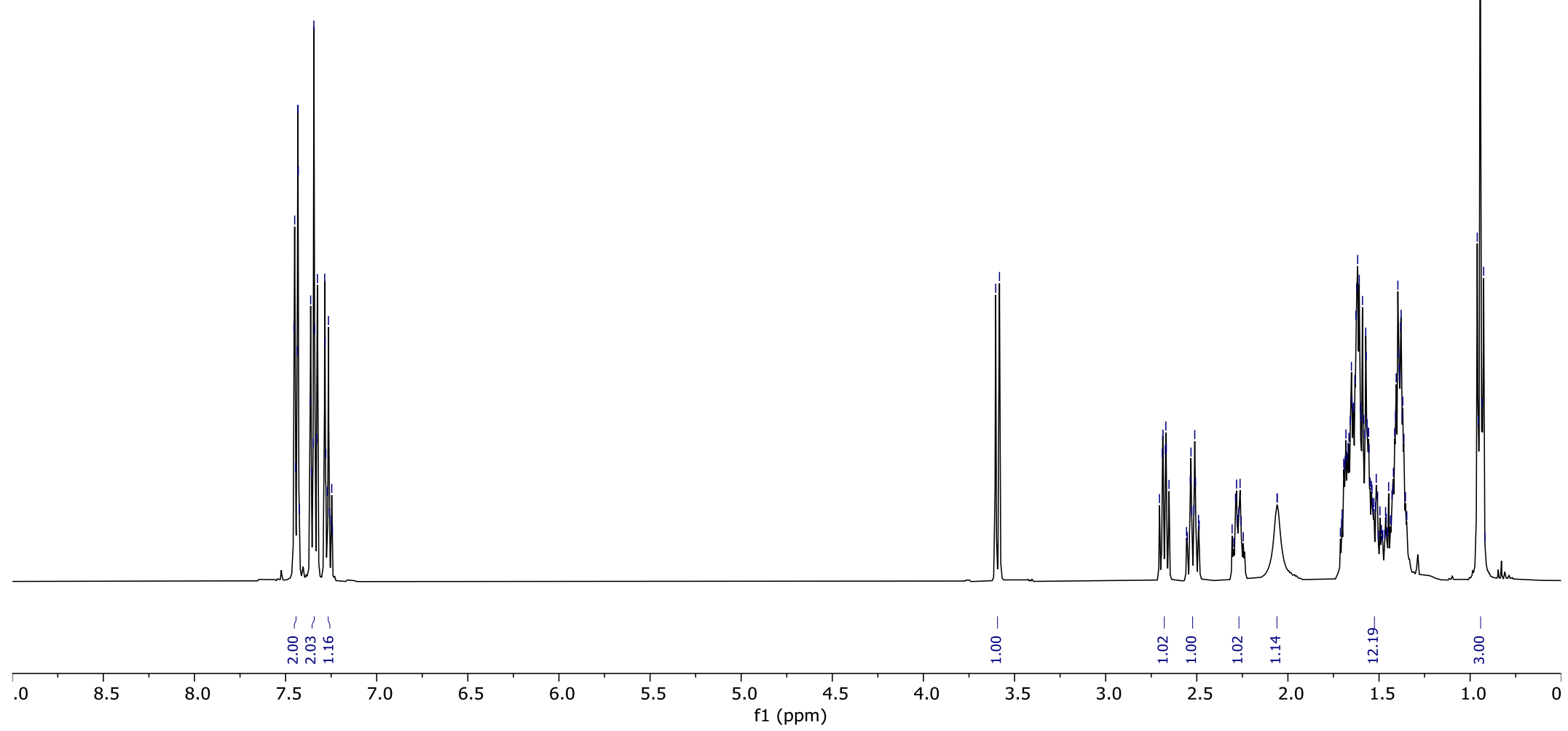




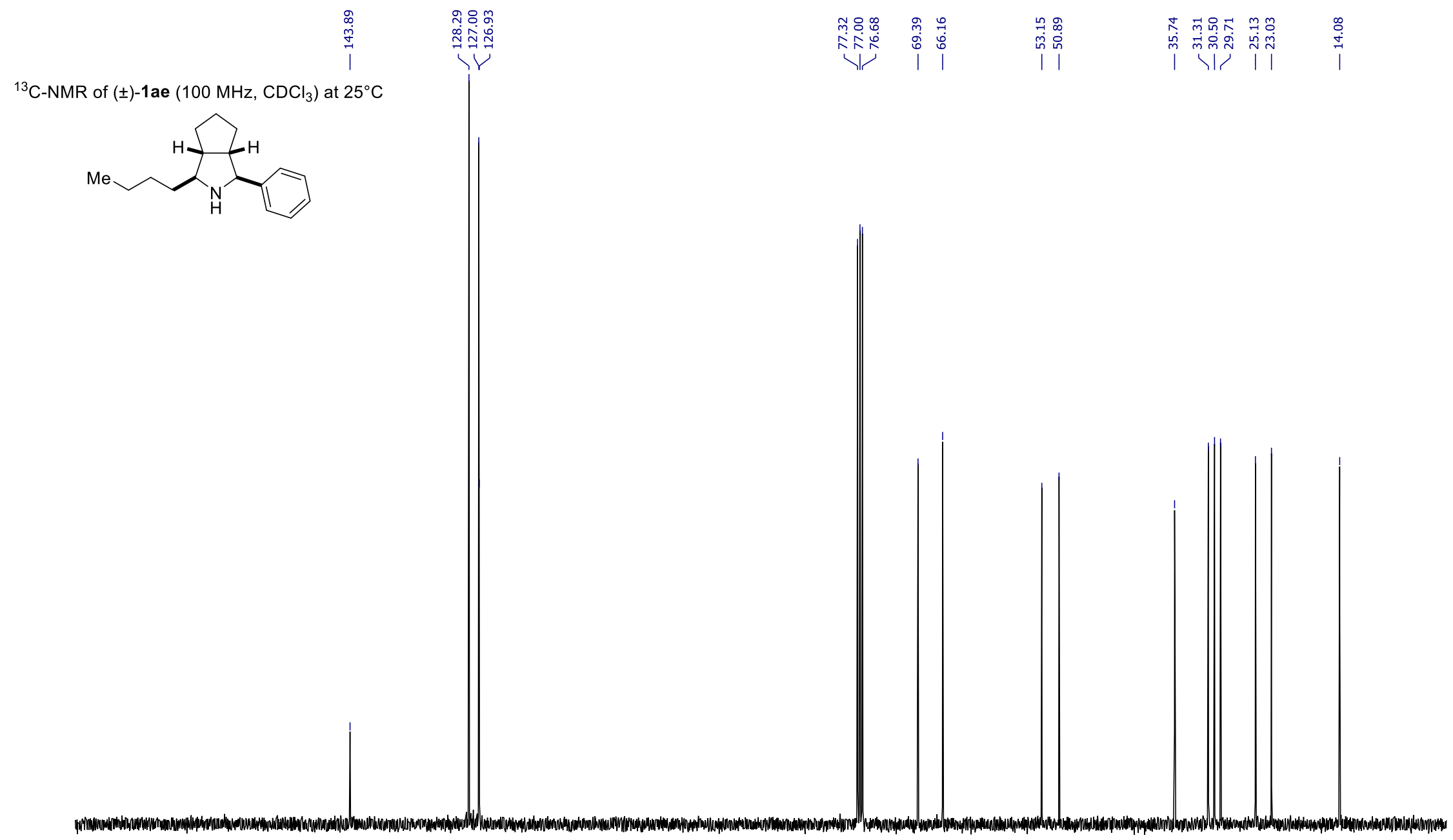

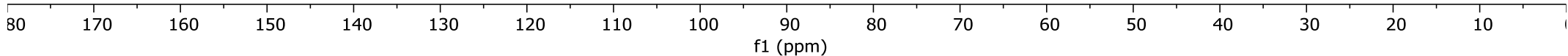




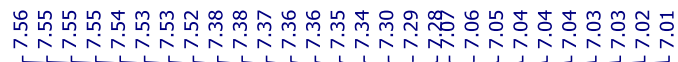

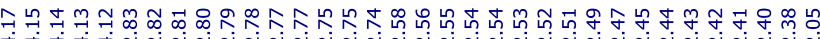

${ }^{1} \mathrm{H}-\mathrm{NMR}$ of $( \pm)-1$ af $\left(400 \mathrm{MHz}, \mathrm{CDCl}_{3}\right)$ at $25^{\circ} \mathrm{C}$
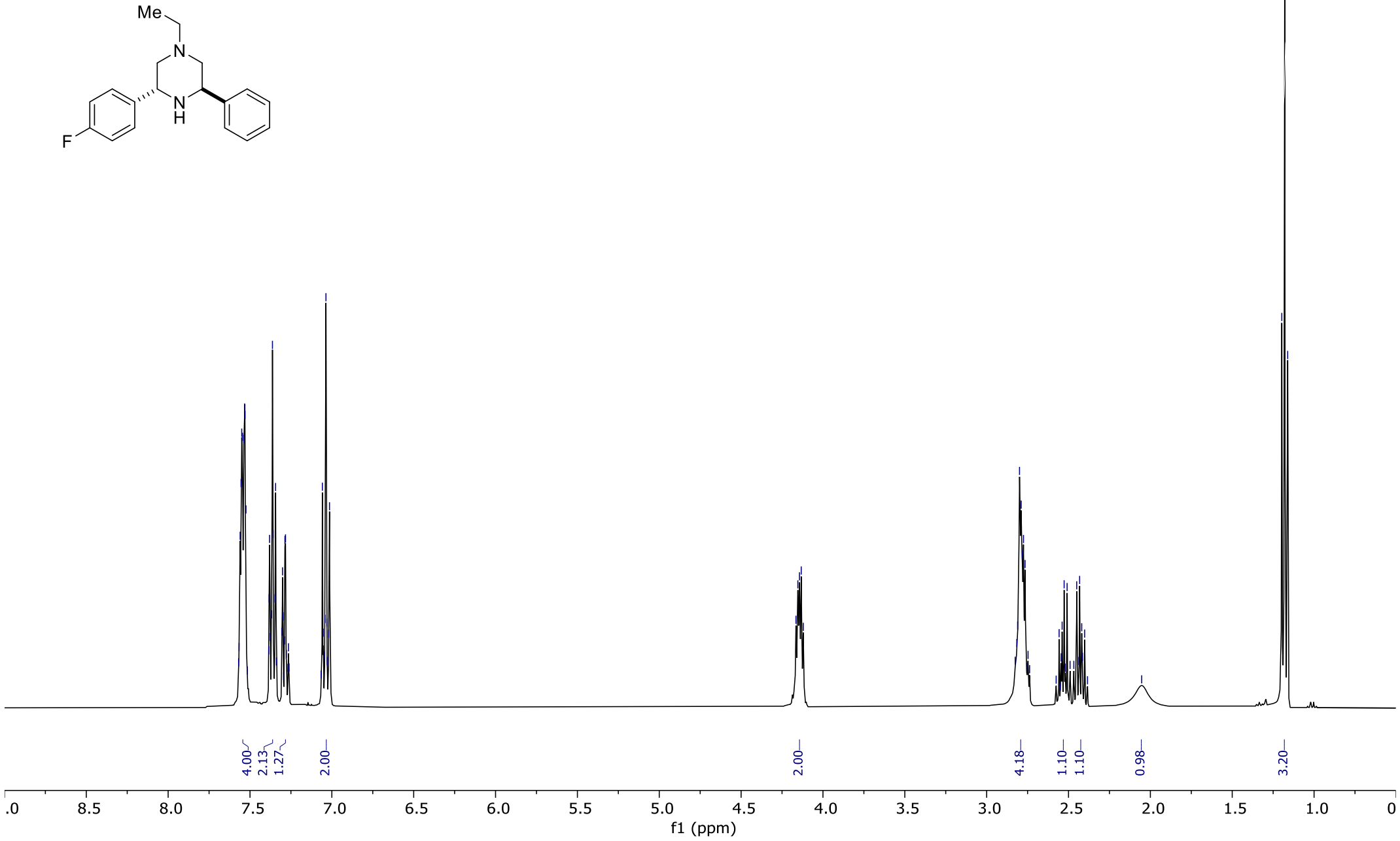


VI

${ }^{13} \mathrm{C}-\mathrm{NMR}$ of $( \pm)-1$ af $\left(100 \mathrm{MHz}, \mathrm{CDCl}_{3}\right)$ at $25^{\circ} \mathrm{C}$
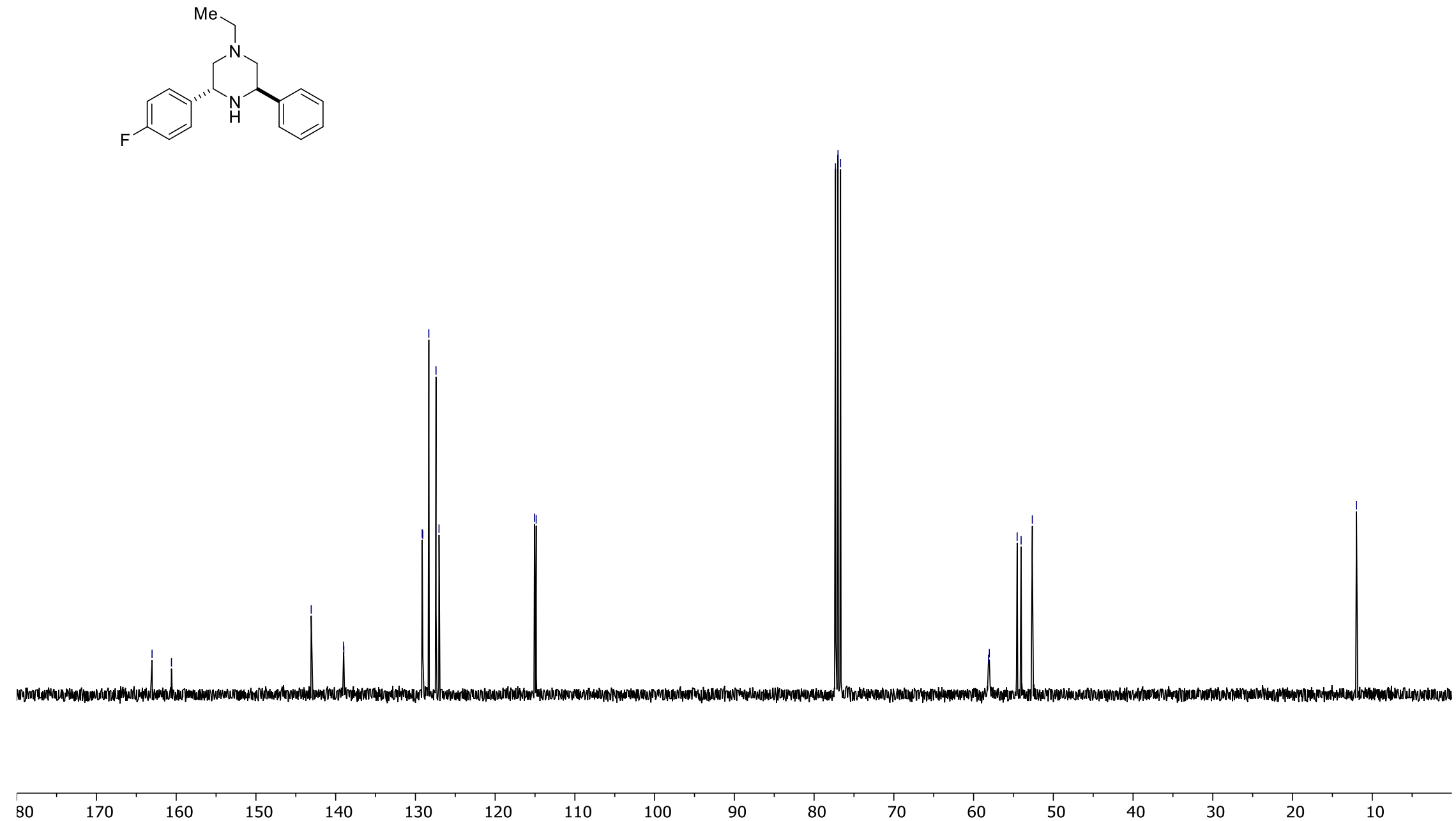
90
$\mathrm{f} 1(\mathrm{ppm})$

$80 \quad 70$

60

50

40

30

20

10

S-142 
${ }^{19} \mathrm{~F}-\mathrm{NMR}$ of $( \pm)-1$ af $\left(377 \mathrm{MHz}, \mathrm{CDCl}_{3}\right)$ at $25^{\circ} \mathrm{C}$

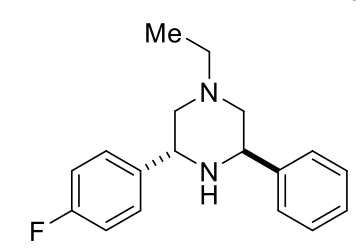

\begin{tabular}{|c|c|c|c|c|c|c|c|c|c|c|c|c|c|c|c|c|c|c|c|}
\hline ) & -10 & -20 & -30 & -40 & -50 & -60 & -70 & -80 & -90 & $\begin{array}{l}-100 \\
\mathrm{f1}(\mathrm{ppm})\end{array}$ & -110 & -120 & -130 & -140 & -150 & -160 & -170 & -180 & -190 \\
\hline
\end{tabular}




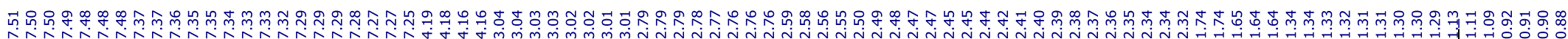
(1)

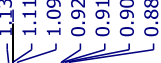

${ }^{1} \mathrm{H}-\mathrm{NMR}$ of $( \pm)-1 \mathbf{a g}\left(400 \mathrm{MHz}, \mathrm{CDCl}_{3}\right)$ at $25^{\circ} \mathrm{C}$<smiles>CCCCCC[C@@H]1CN(CC)CC(c2ccccc2)N1</smiles>
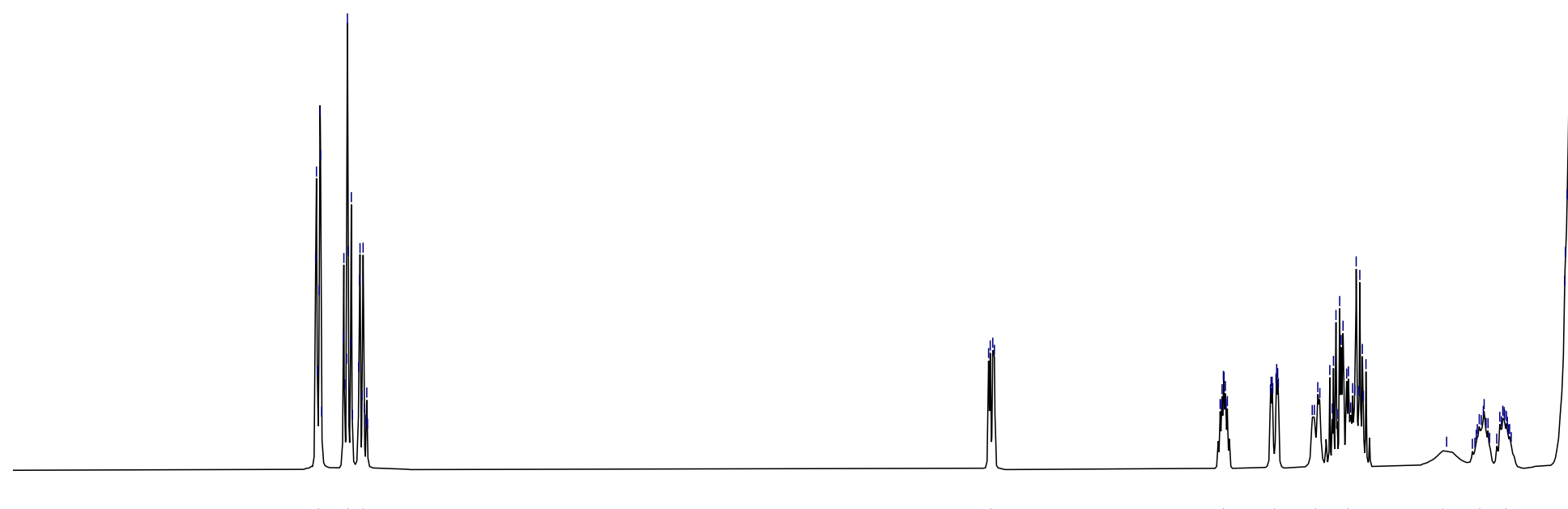

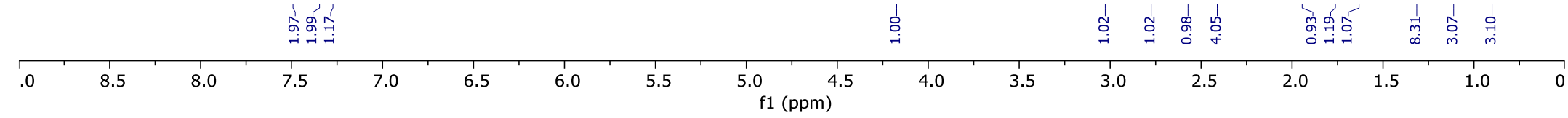

S-144 


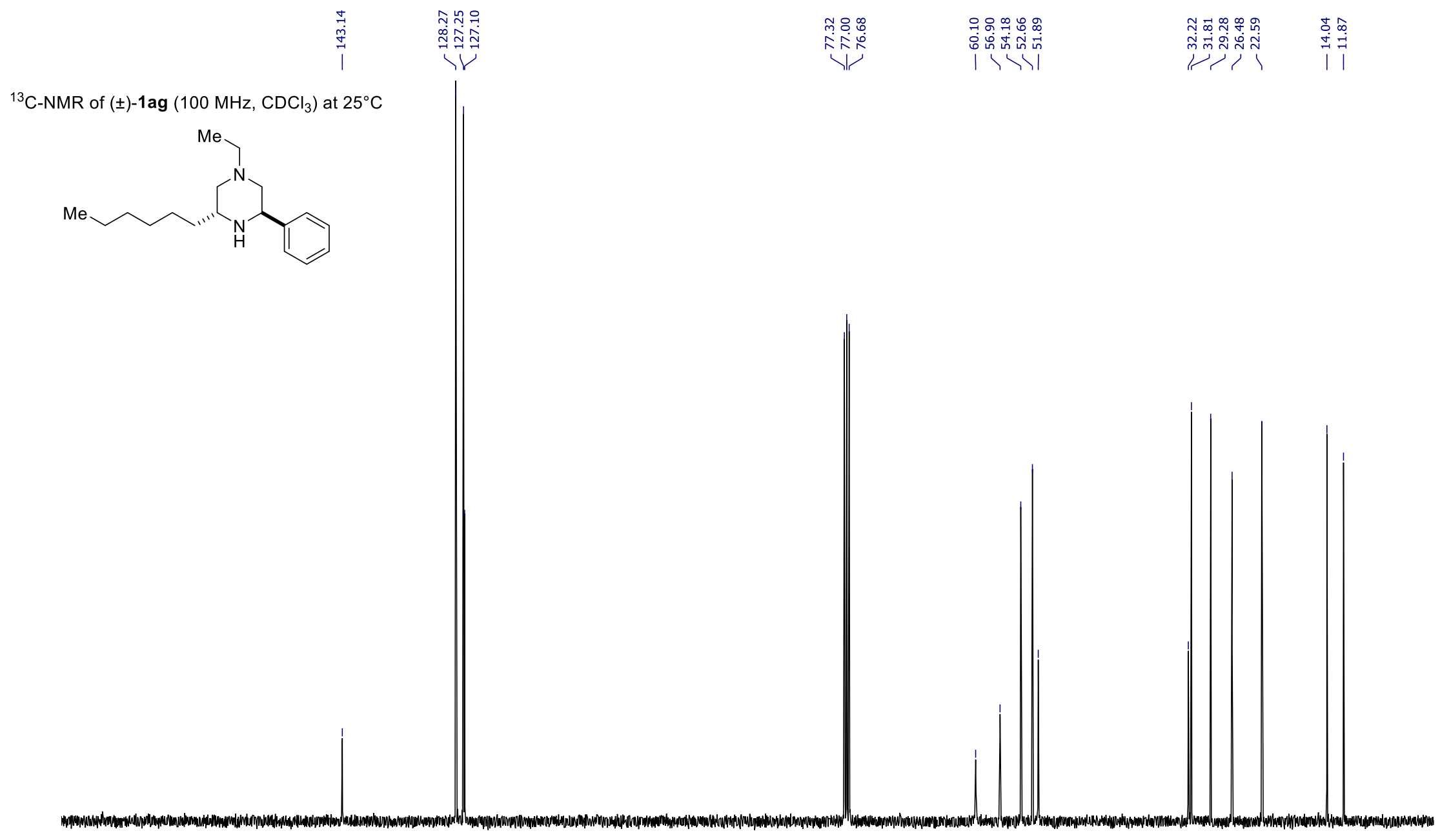

\begin{tabular}{|c|c|c|c|c|c|c|c|c|c|c|c|c|c|c|c|c|}
\hline 170 & 160 & 150 & 140 & 130 & 120 & 110 & 100 & $\begin{array}{c}90 \\
\text { f1 }(\mathrm{ppm})\end{array}$ & 80 & 70 & 60 & 50 & 40 & 30 & 20 & 10 \\
\hline
\end{tabular}




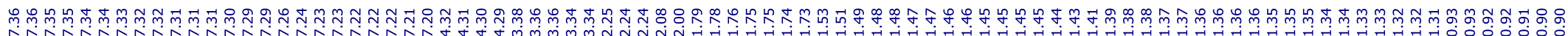

${ }^{1} \mathrm{H}-\mathrm{NMR}$ of $( \pm)-1 \mathrm{ah}\left(400 \mathrm{MHz}, \mathrm{CDCl}_{3}\right)$ at $25^{\circ} \mathrm{C}$<smiles>CCCCC1CC[C@@H](c2ccccc2)N1</smiles>

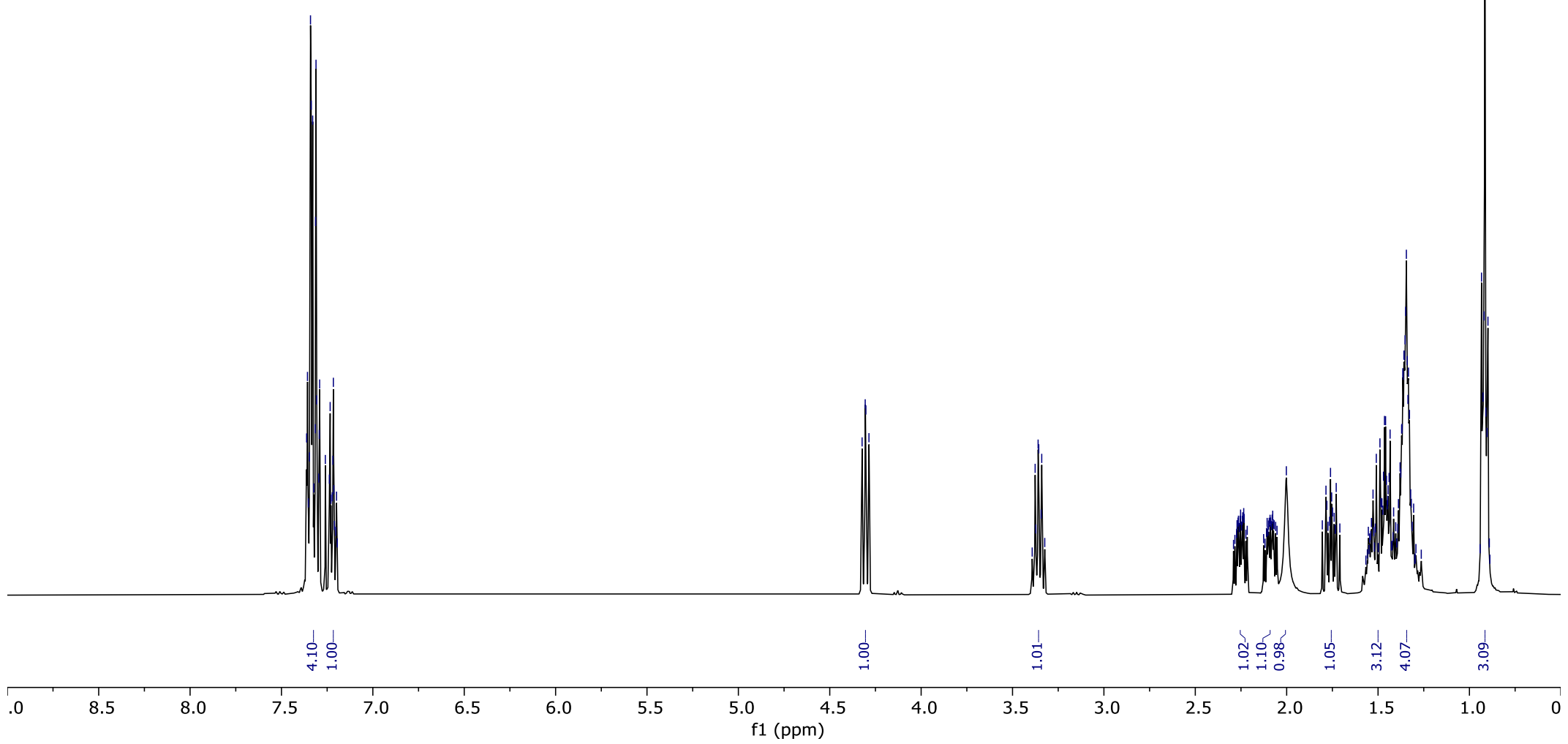

S-146 

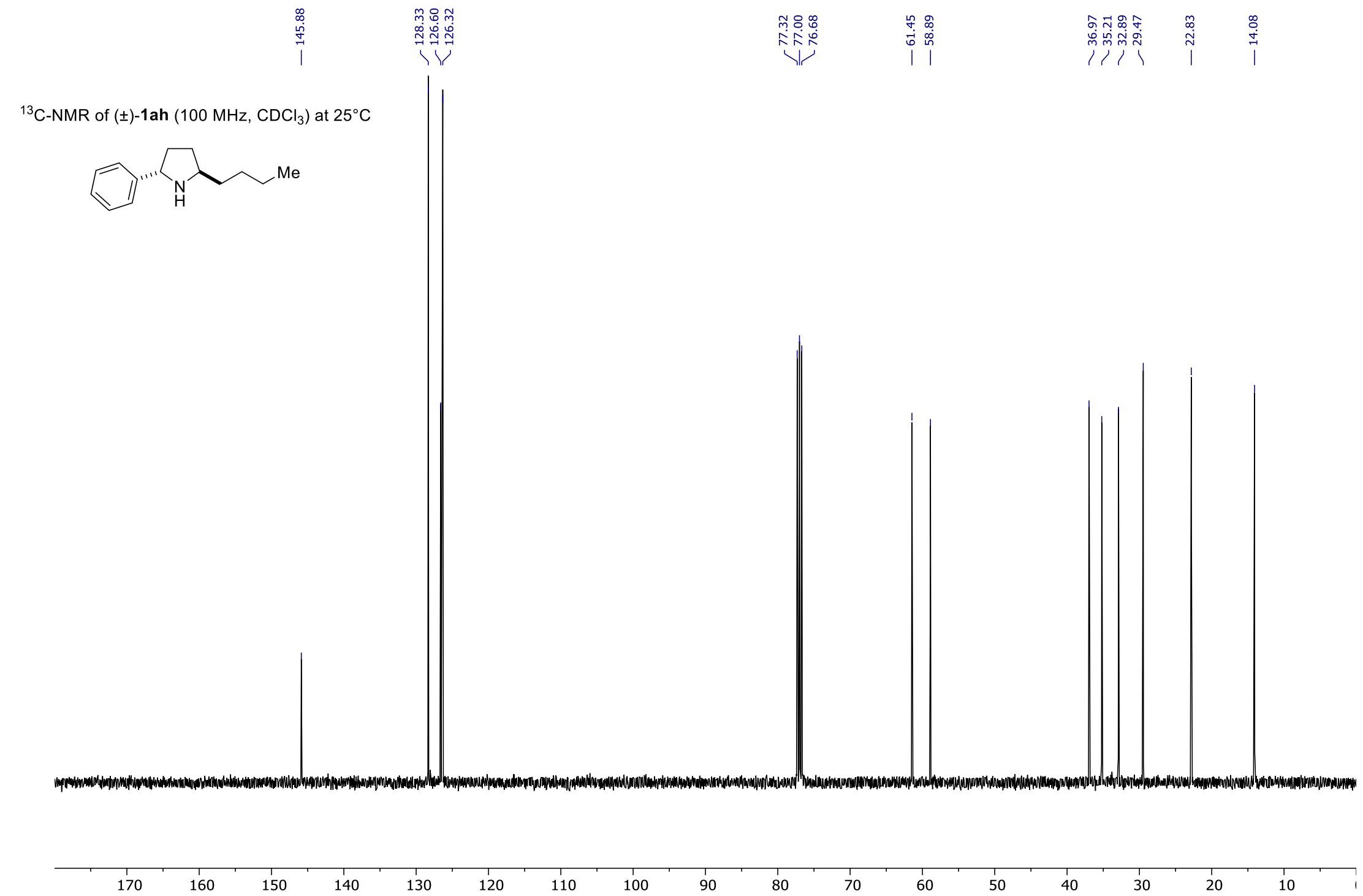

150

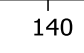

130

120

110

100

90
$\mathrm{f} 1(\mathrm{ppm})$

80

70

60

50

40

30

20

10

S-147 


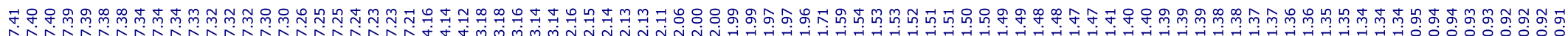

${ }^{1} \mathrm{H}-\mathrm{NMR}$ of $( \pm)-1 \mathrm{ah} '\left(400 \mathrm{MHz}, \mathrm{CDCl}_{3}\right)$ at $25^{\circ} \mathrm{C}$<smiles>CCCCC1CCC(c2ccccc2)N1</smiles>

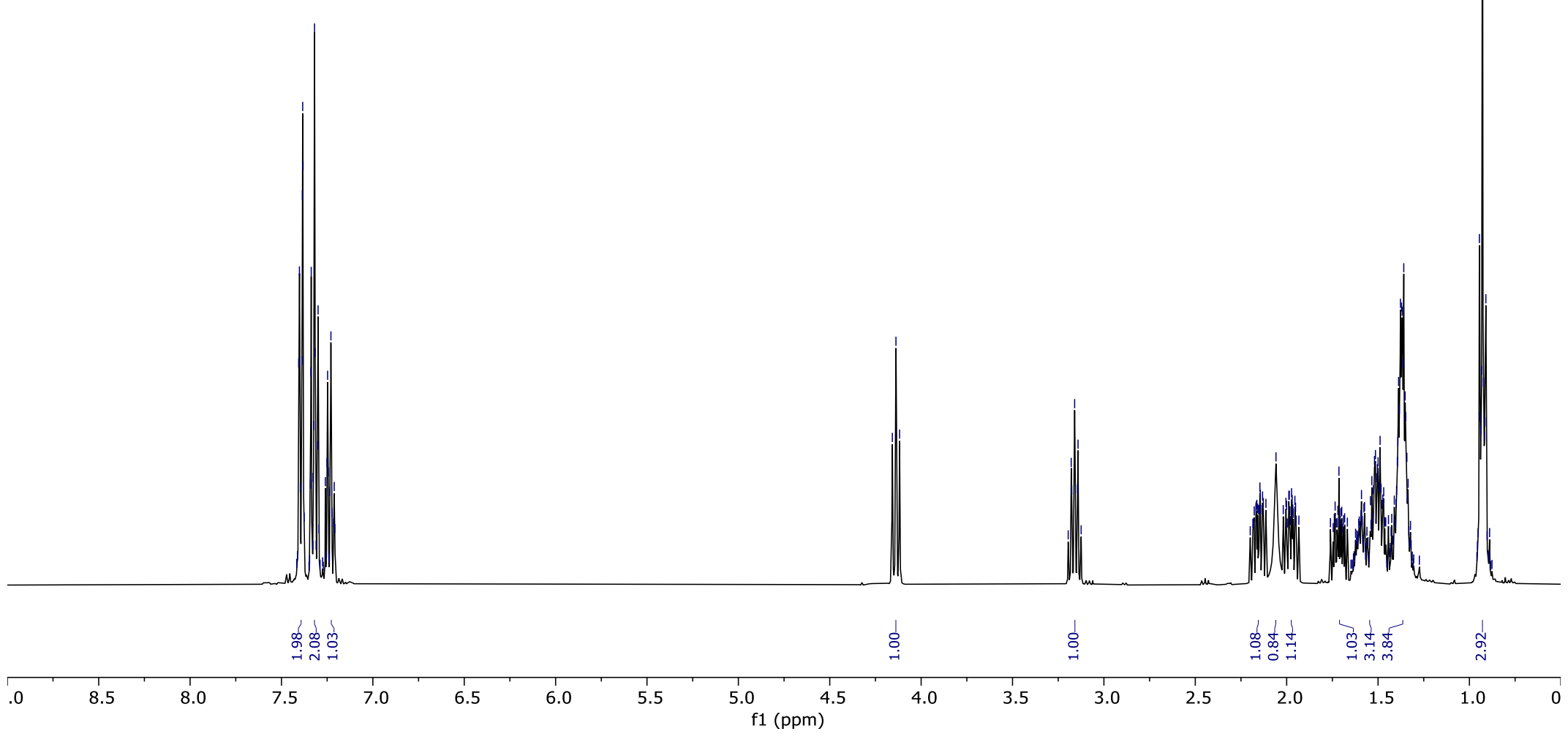

S-148 

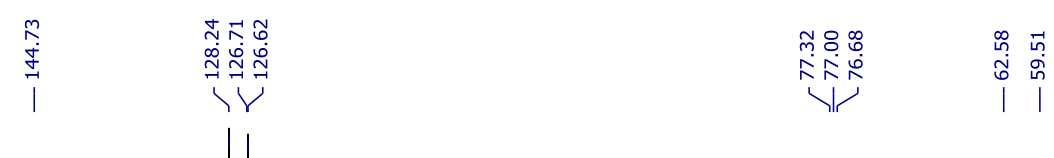

|

${ }^{13} \mathrm{C}-\mathrm{NMR}$ of $( \pm)-1 \mathrm{ah}^{\prime}\left(100 \mathrm{MHz}, \mathrm{CDCl}_{3}\right)$ at $25^{\circ} \mathrm{C}$<smiles>CCCCC1CCC(c2ccccc2)N1</smiles>

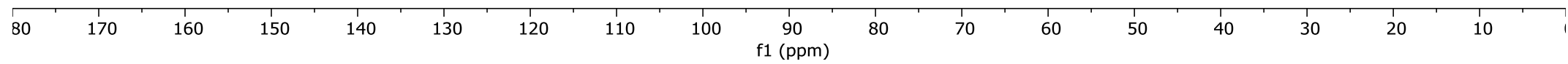




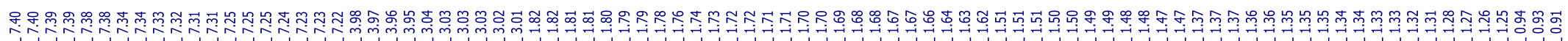

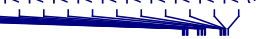

${ }^{1} \mathrm{H}-\mathrm{NMR}$ of $( \pm)-1$ ai $\left(500 \mathrm{MHz}, \mathrm{CDCl}_{3}\right)$ at $25^{\circ} \mathrm{C}$<smiles>CCCC[C@@H]1CCCC(c2ccccc2)N1</smiles>

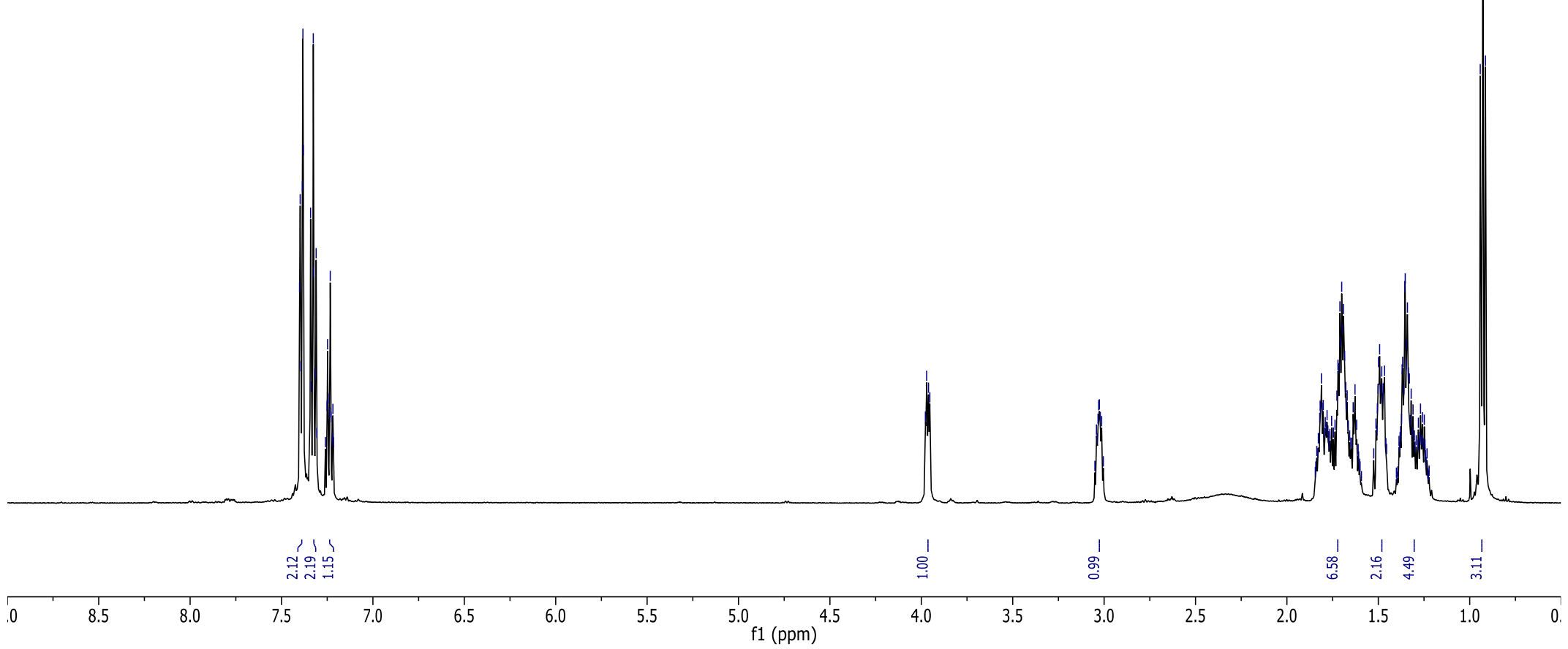

S-150 


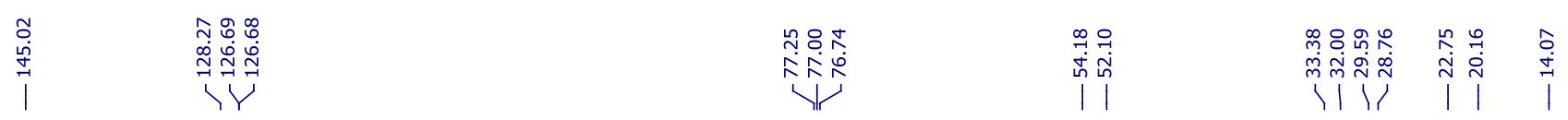

${ }^{13} \mathrm{C}-\mathrm{NMR}$ of $( \pm)-1$ ai $\left(125 \mathrm{MHz}, \mathrm{CDCl}_{3}\right)$ at $25^{\circ} \mathrm{C}$
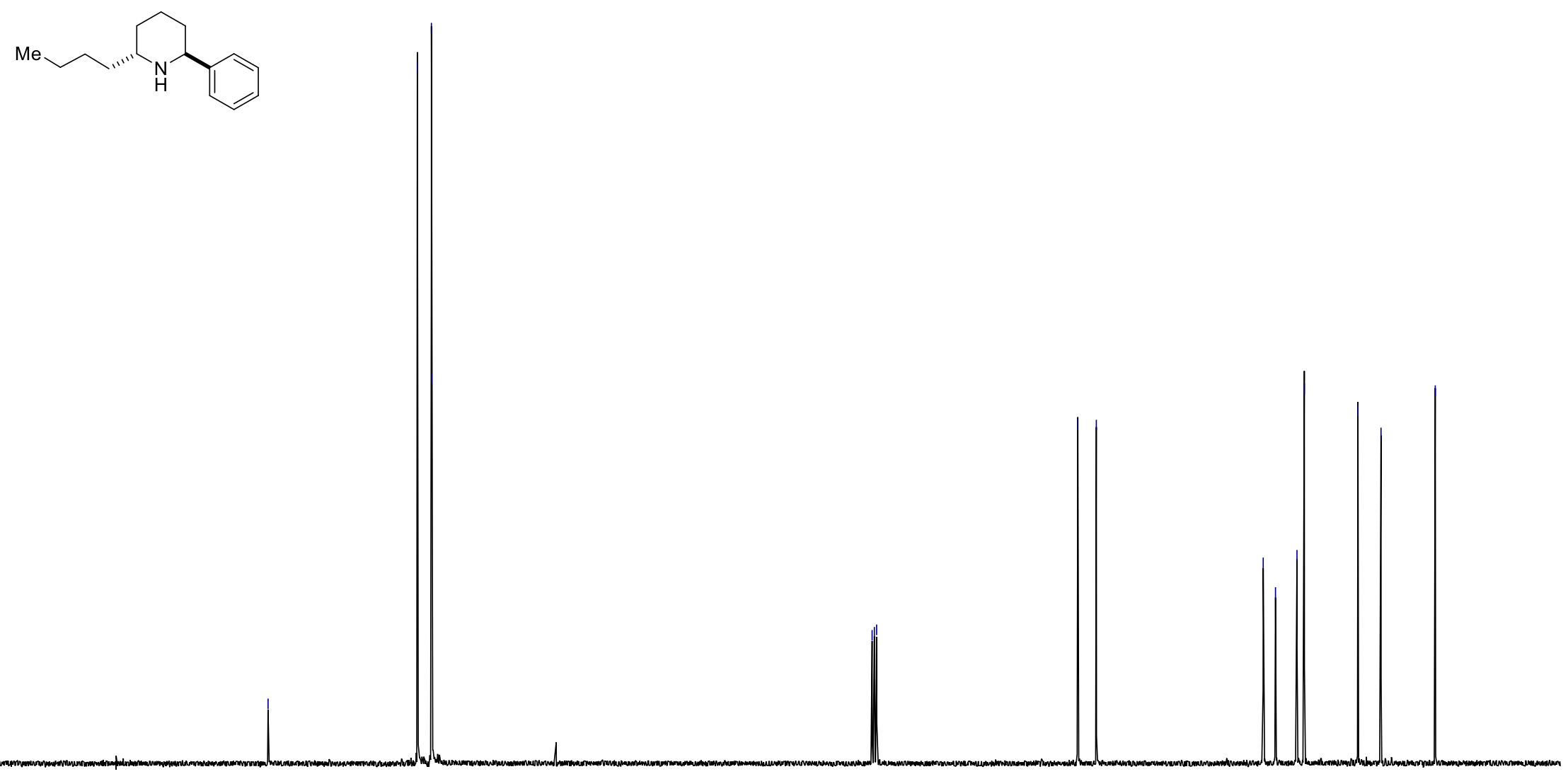


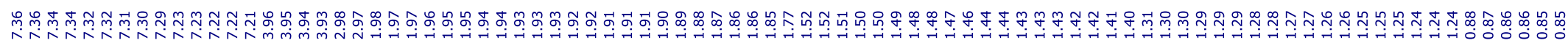

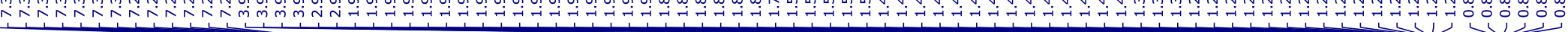

${ }^{1} \mathrm{H}-\mathrm{NMR}$ of $( \pm)-1$ aj $\left(500 \mathrm{MHz}, \mathrm{CDCl}_{3}\right)$ at $25^{\circ} \mathrm{C}$

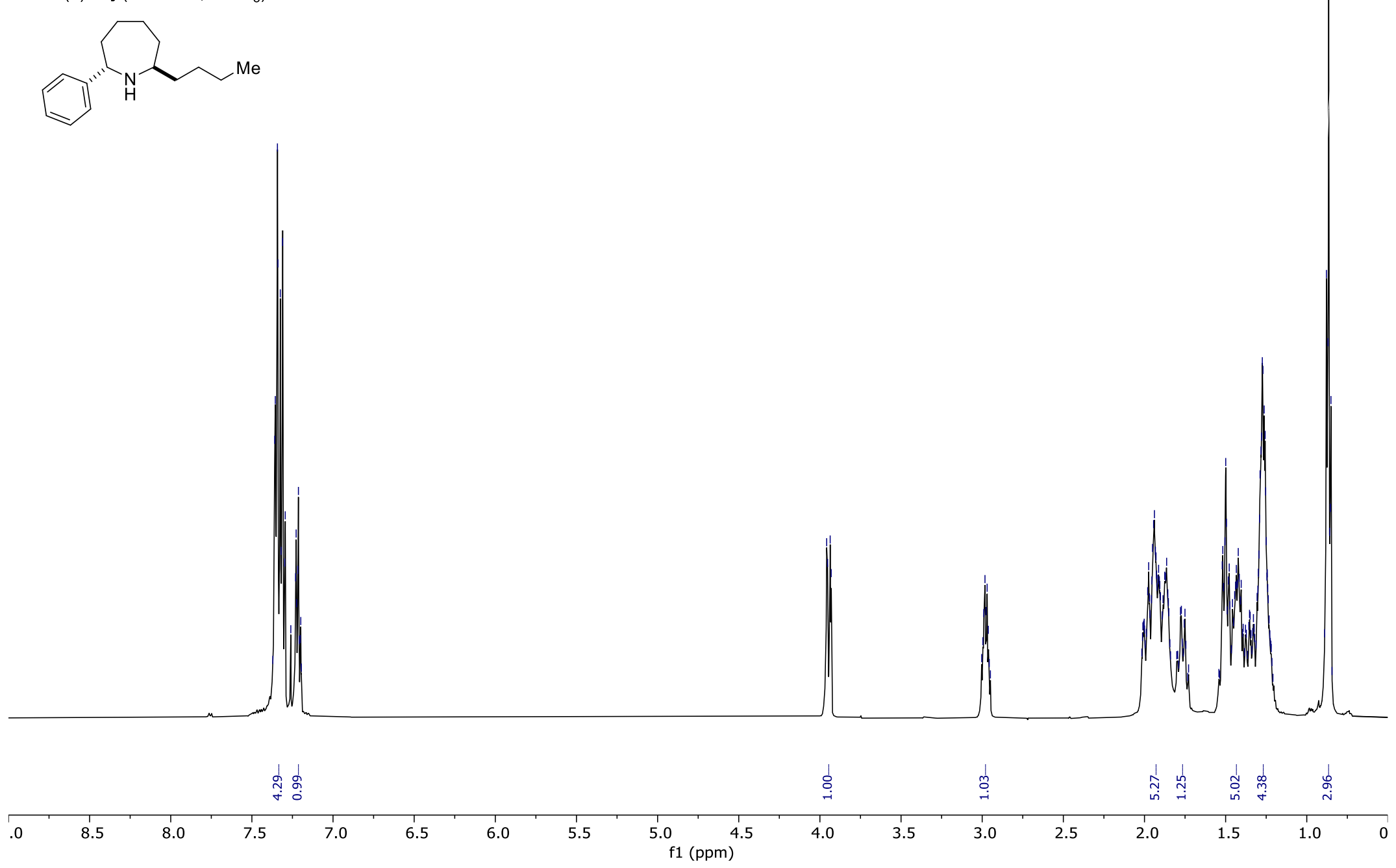

S-152 


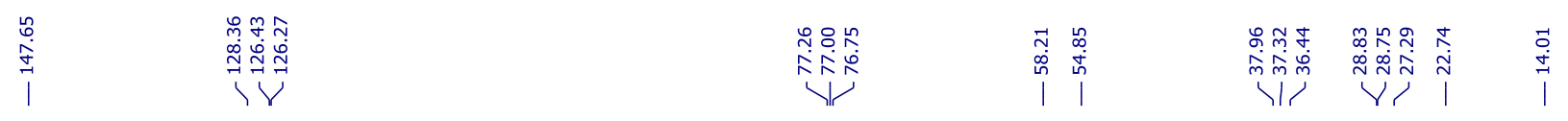

${ }^{13} \mathrm{C}-\mathrm{NMR}$ of $( \pm)-1$ aj $\left(125 \mathrm{MHz}, \mathrm{CDCl}_{3}\right)$ at $25^{\circ} \mathrm{C}$<smiles>CCCCC1CCCC[C@@H](c2ccccc2)N1</smiles>

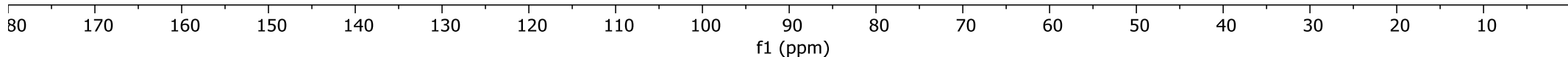




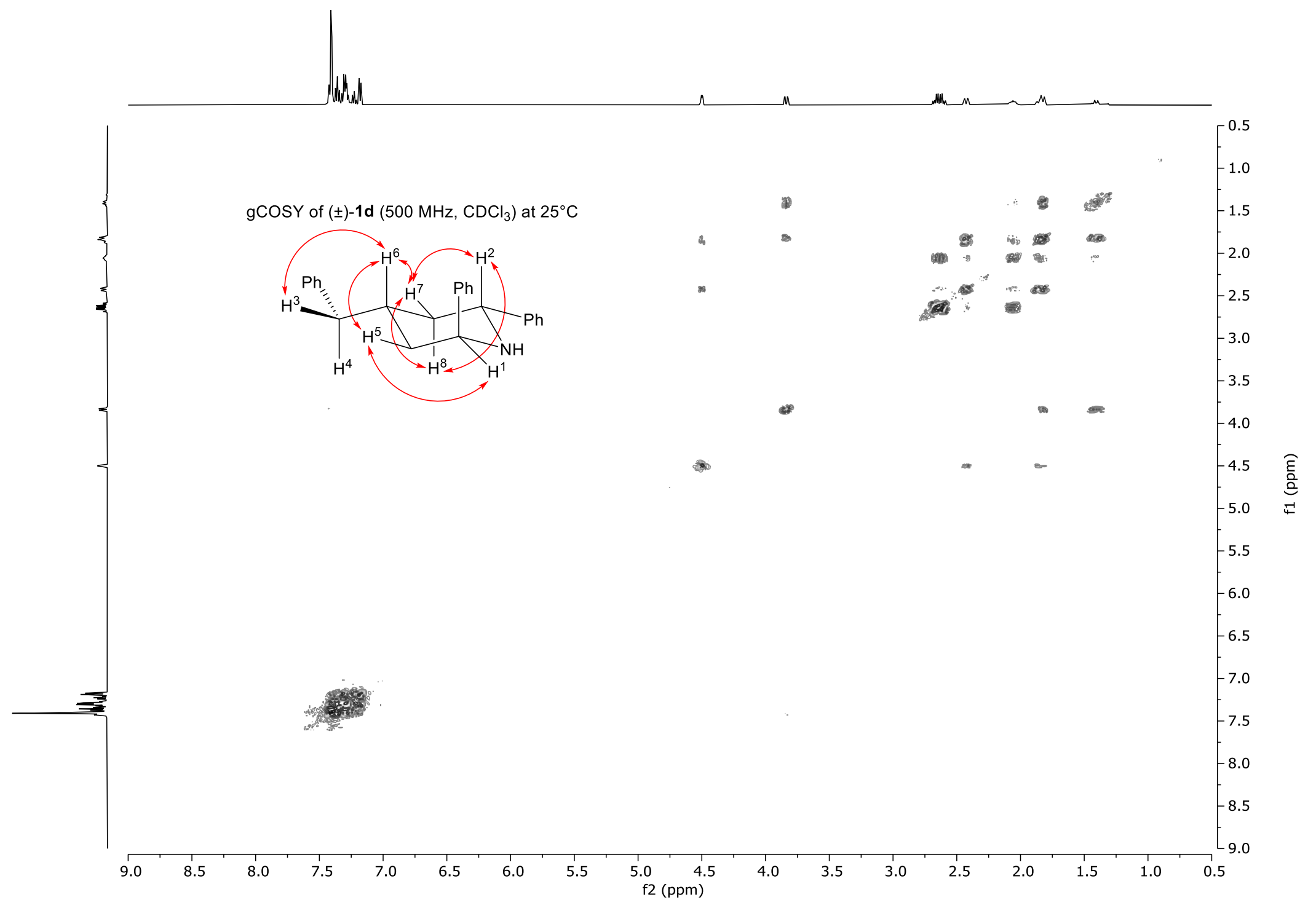

S-154 


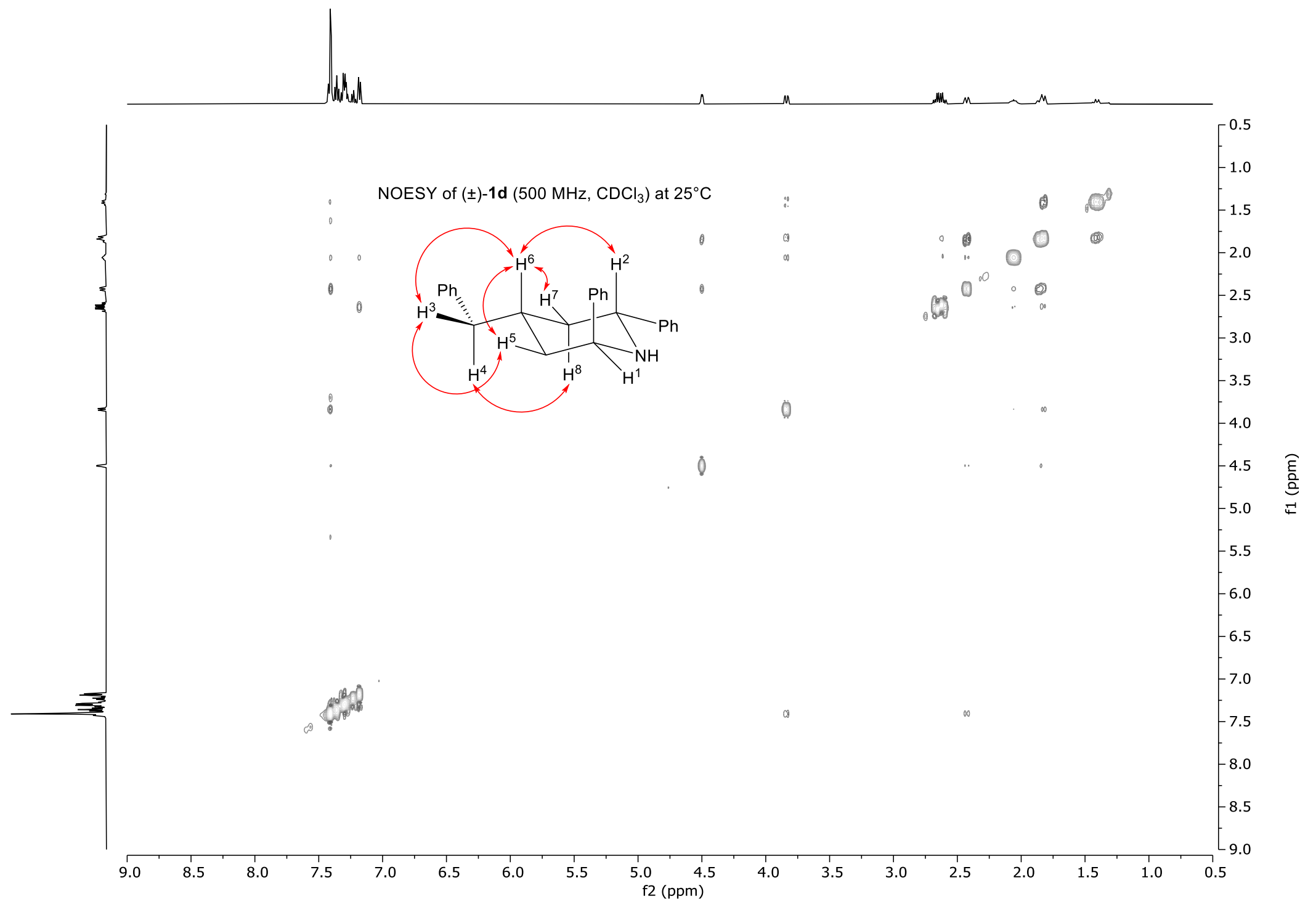

S-155 


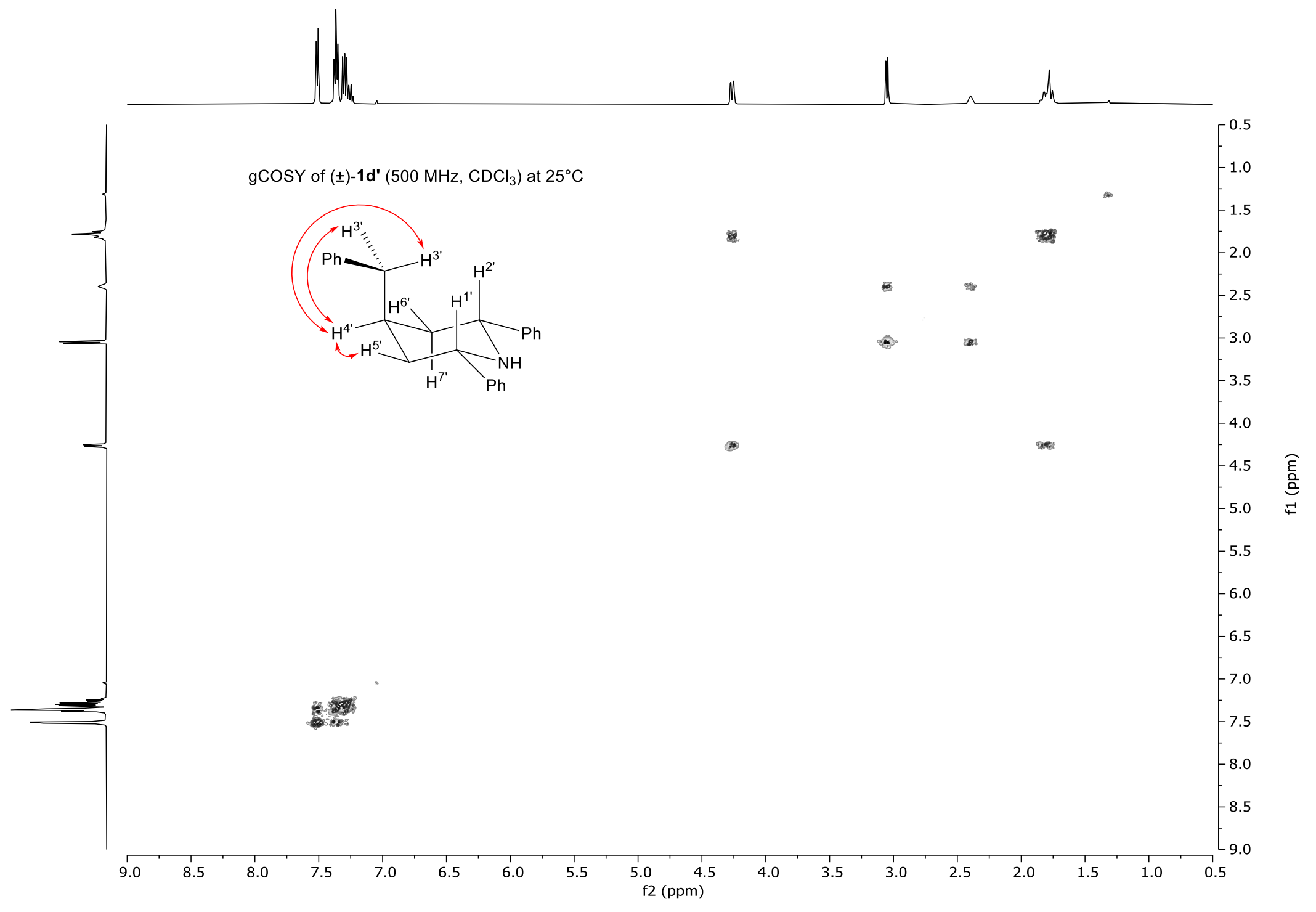

S-156 


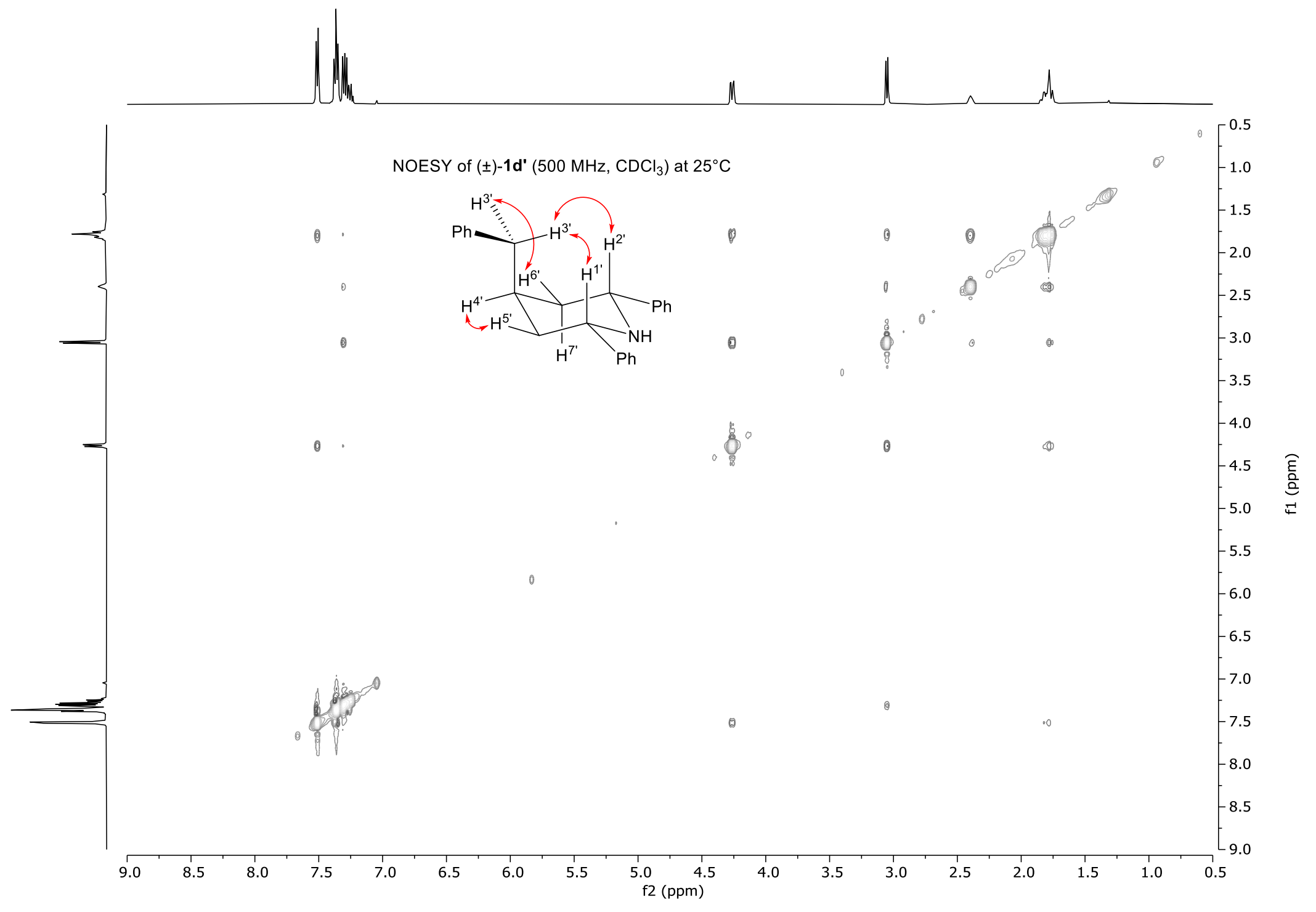

S-157 


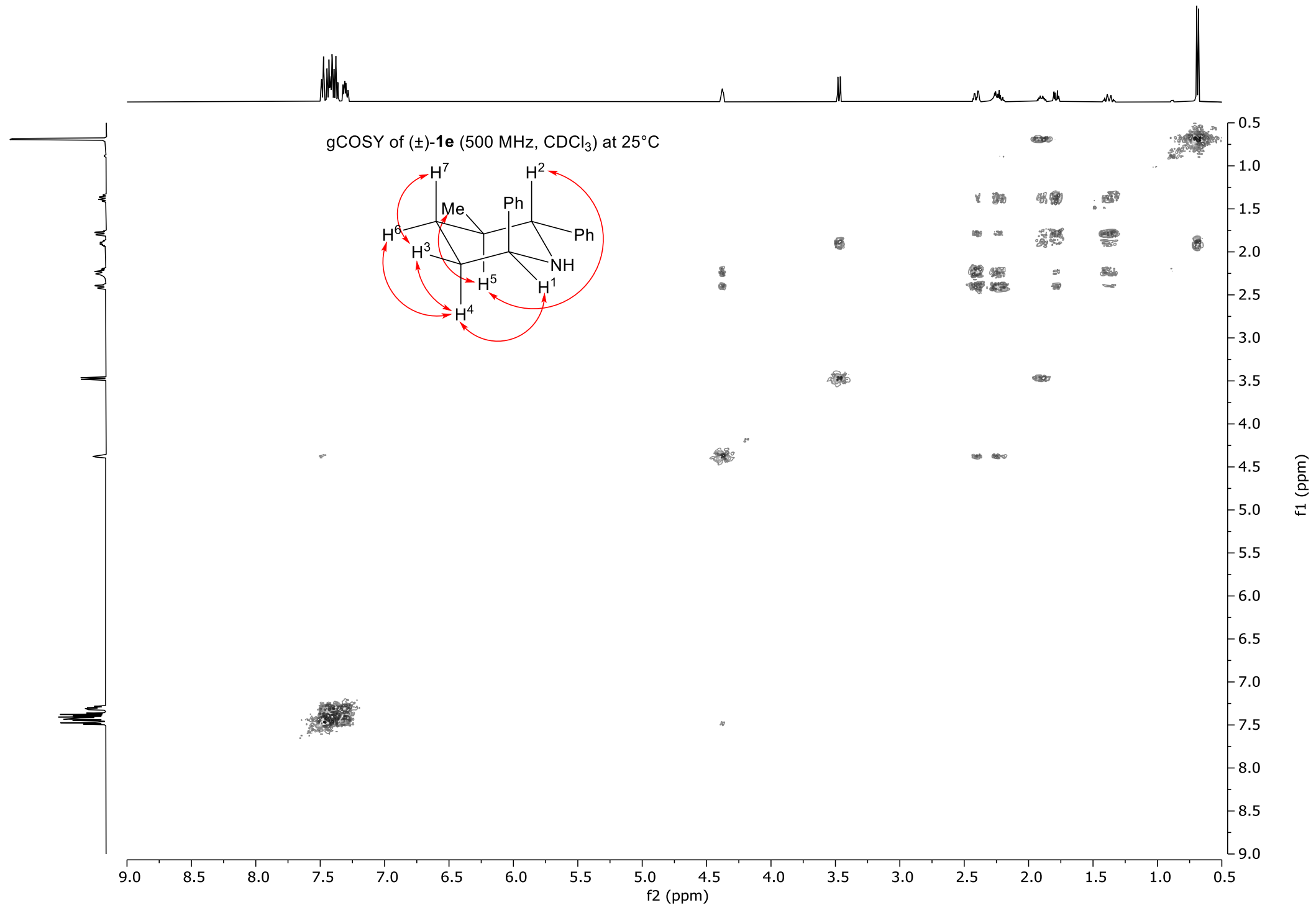

S-158 


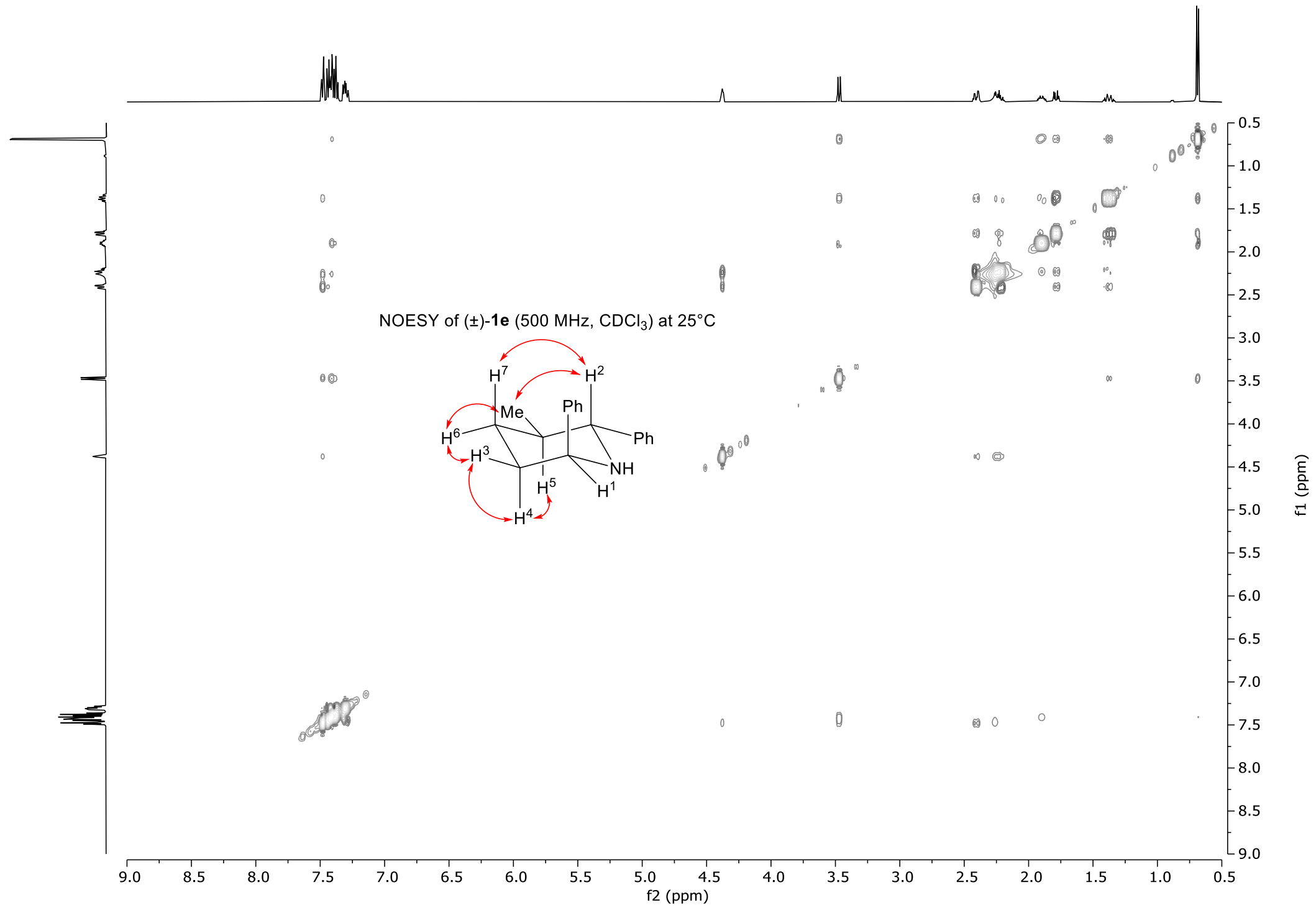

S-159 


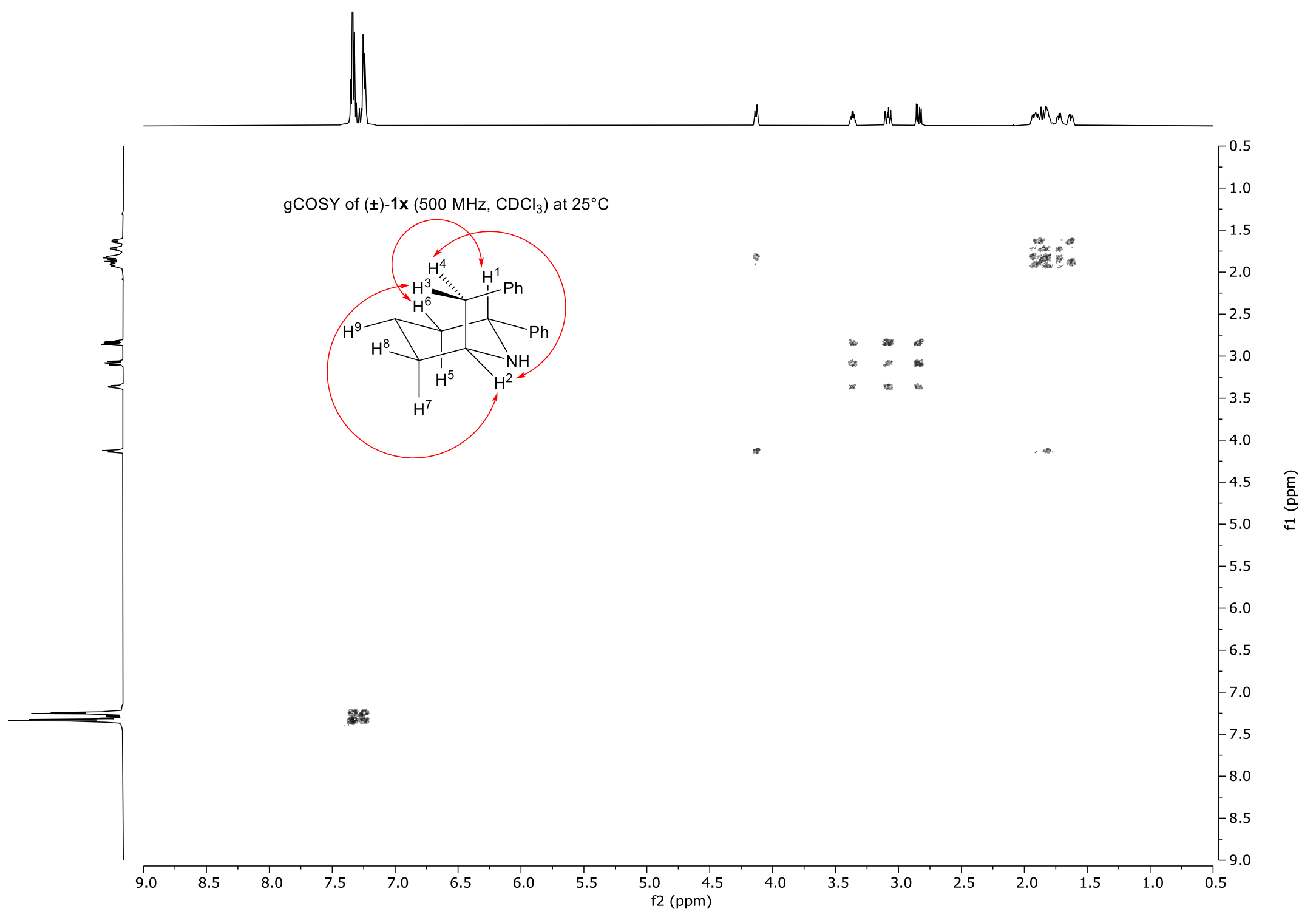

S-160 


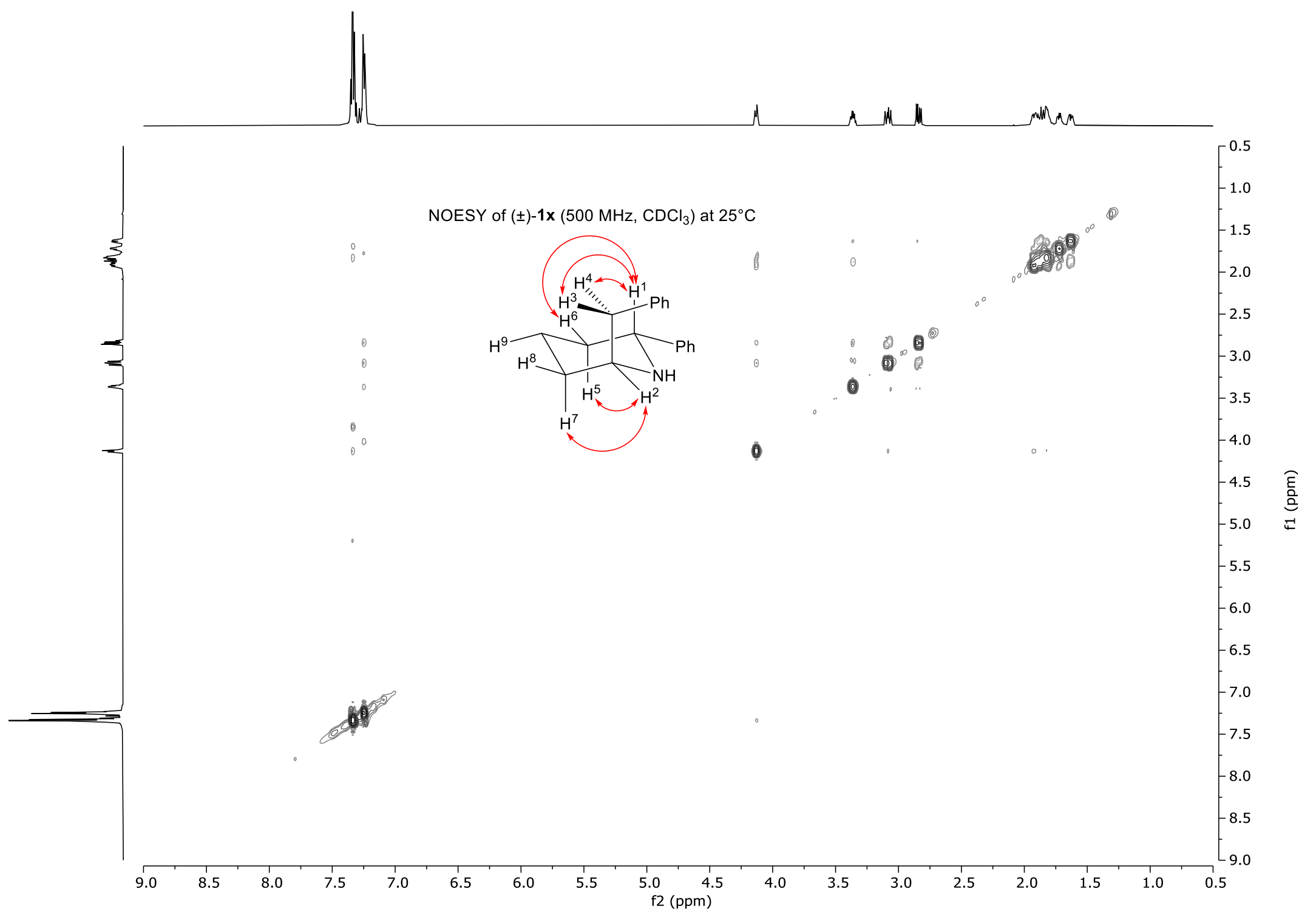

S-161 


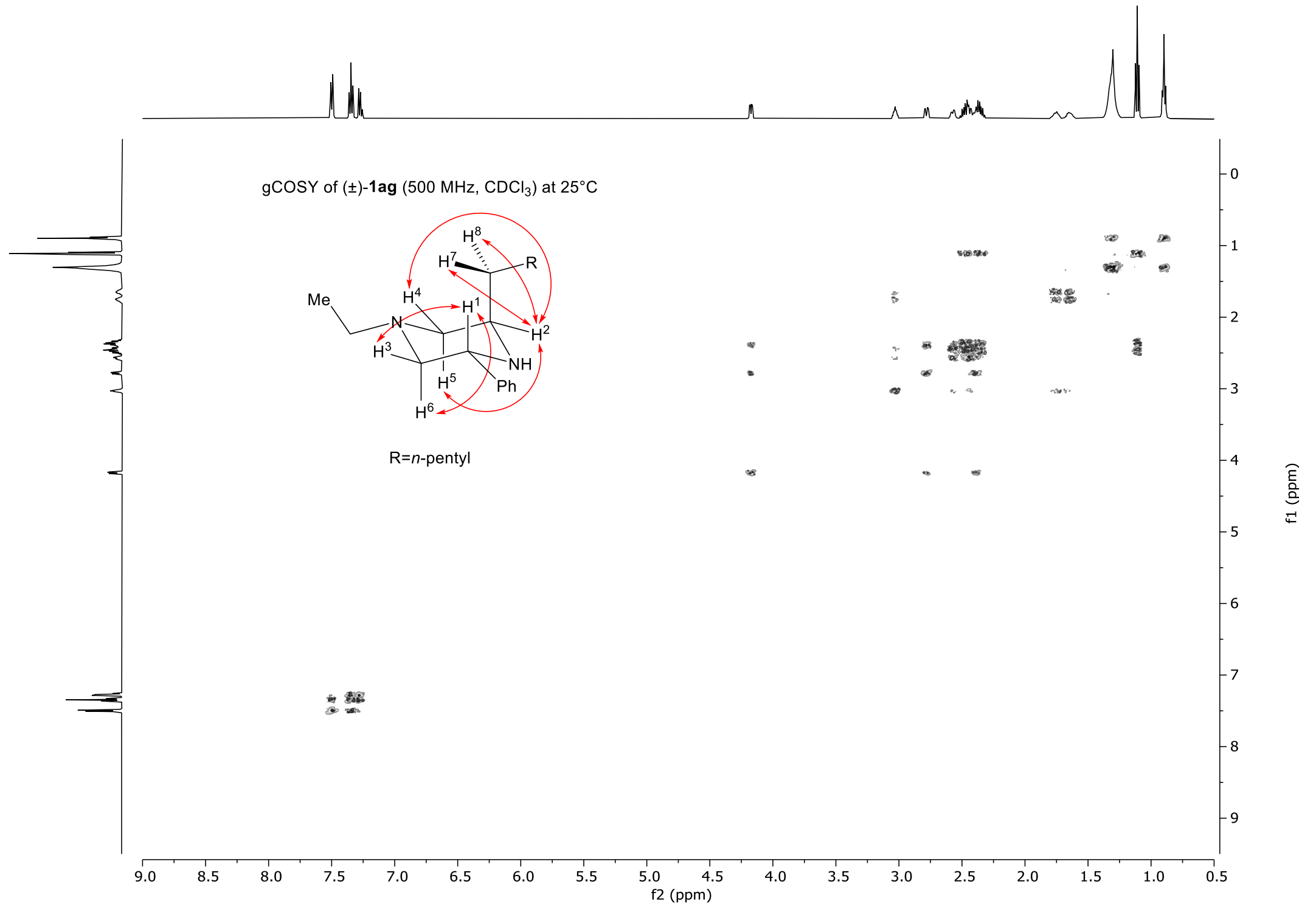




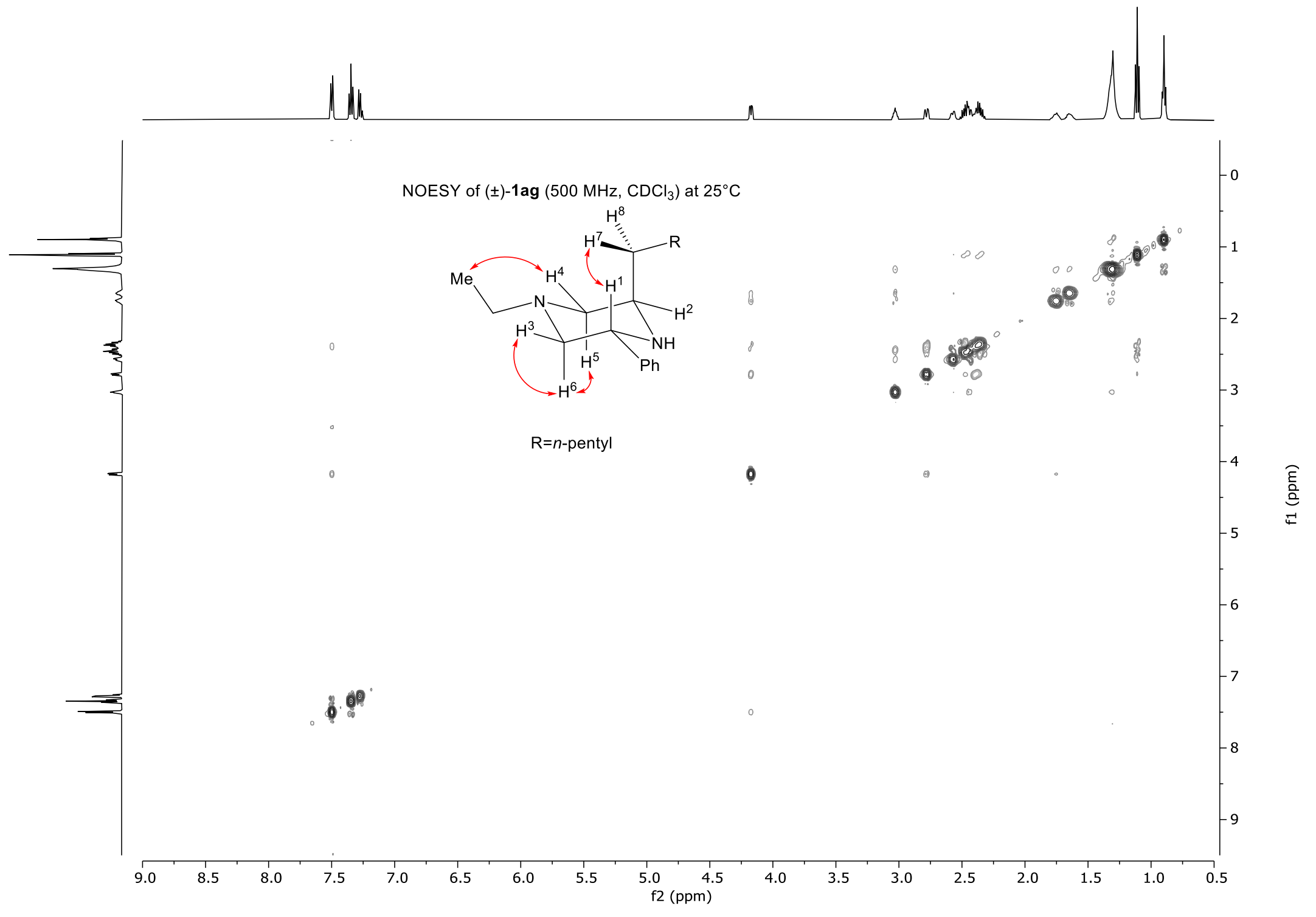




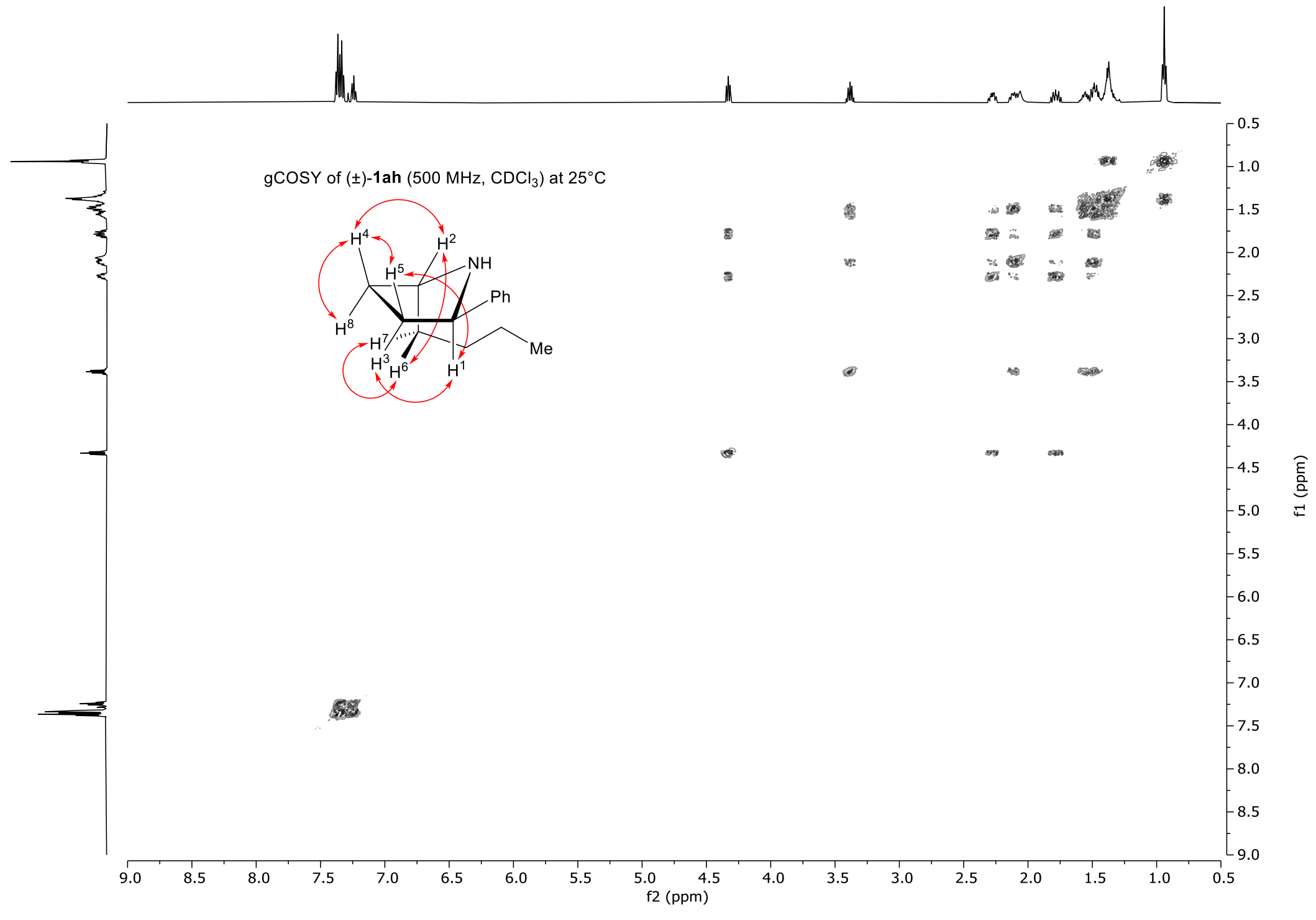

S-164 


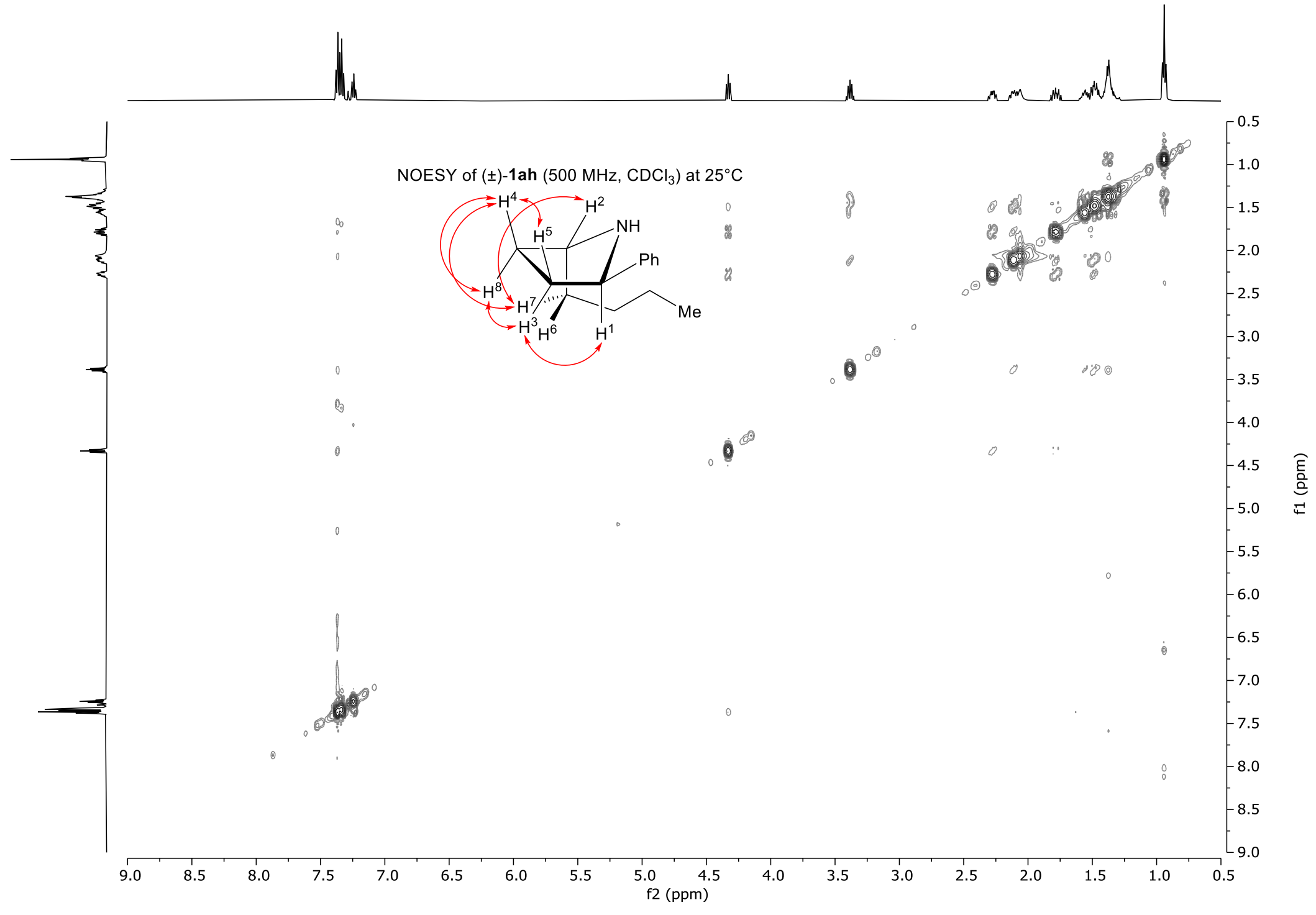

S-165 


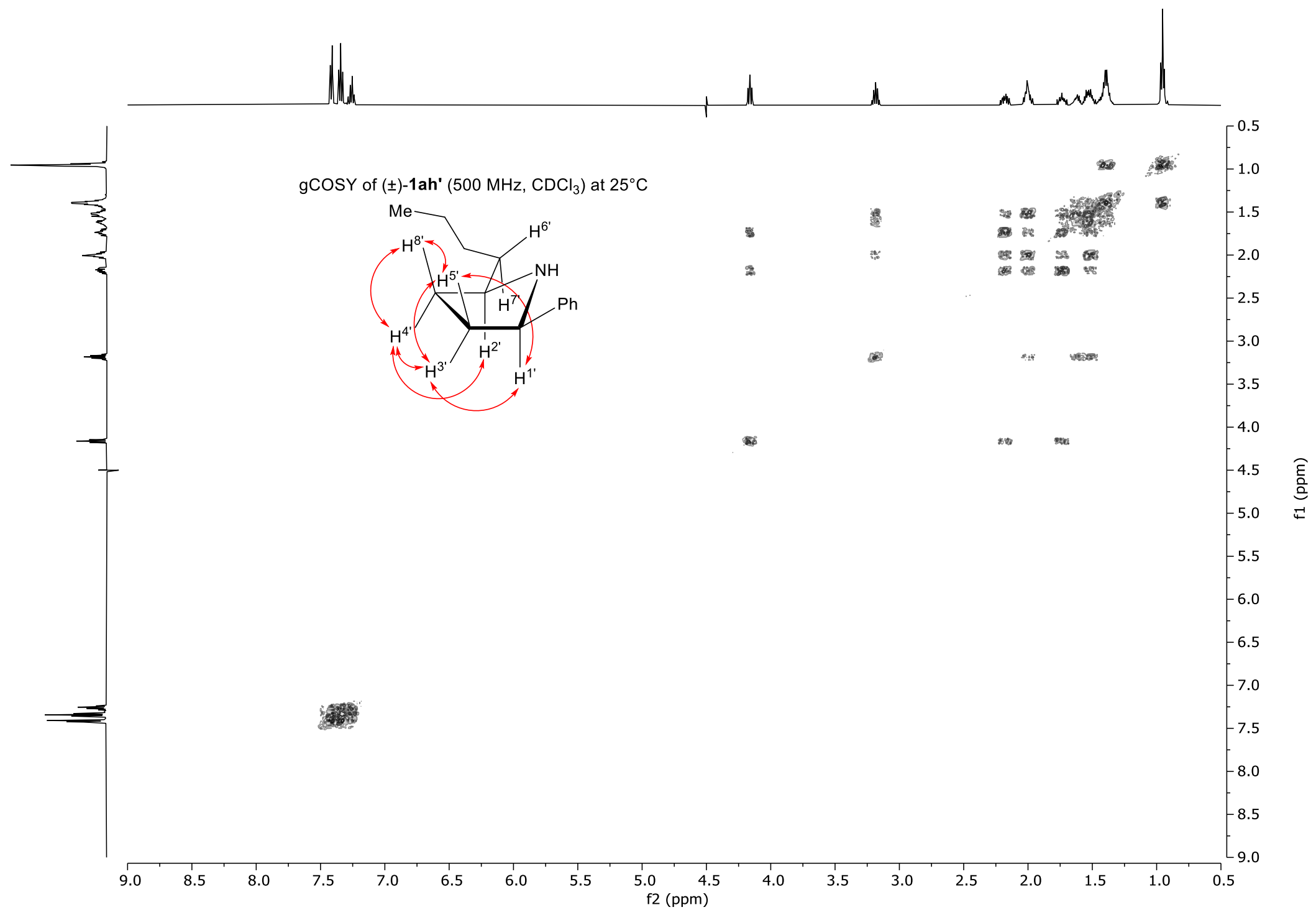




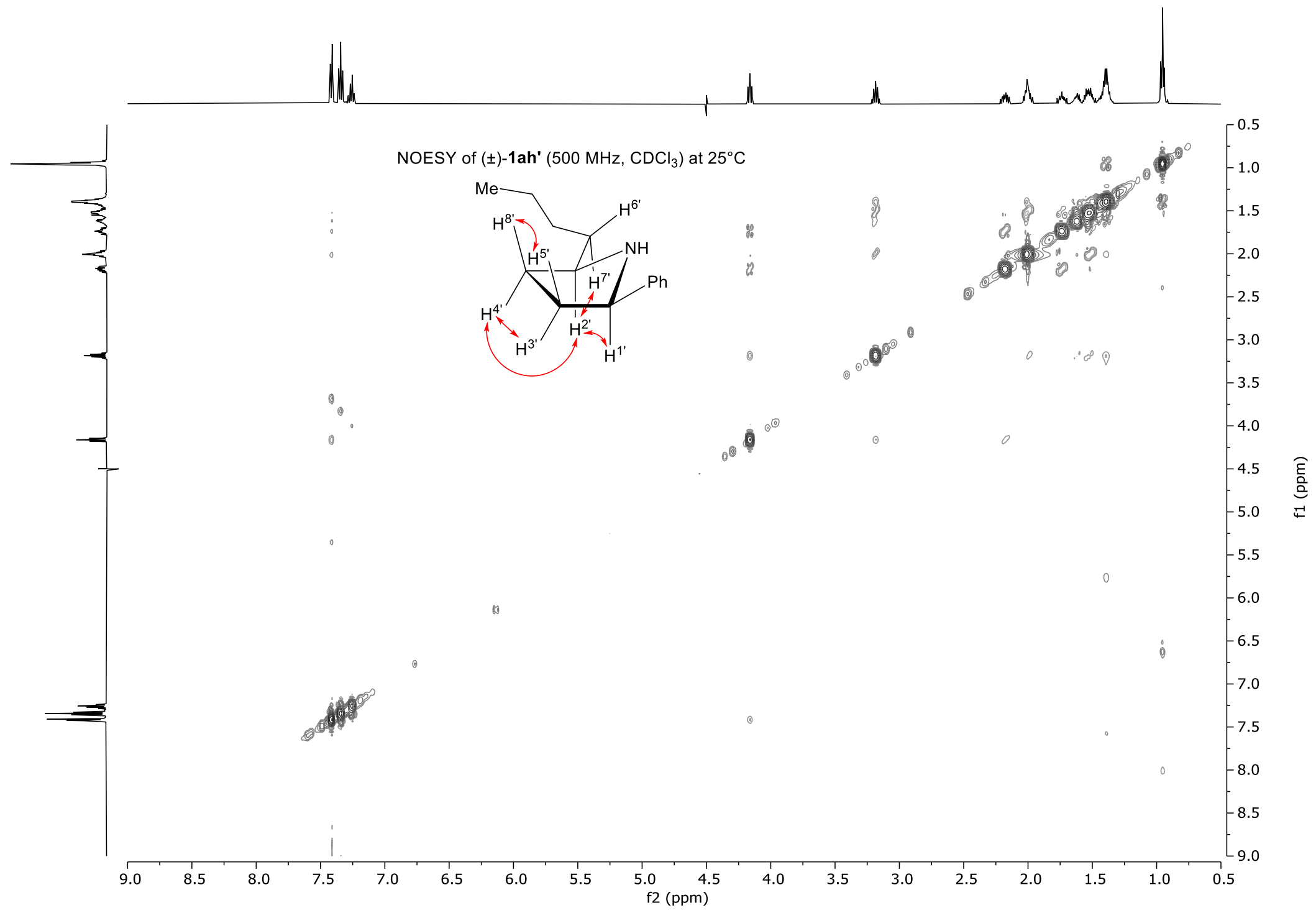

